Universidad Politécnica de Madrid

Escuela Técnica Superior de Ingenieros Industriales

\title{
THEORETICAL AND EXPERIMENTAL STUDY OF THE FATIGUE BEHAVIOR OF LARGE PASSENGER TRANSPORT VEHICLES \\ UNDER DYNAMIC OPERATING LOADS
}

\author{
Doctoral Thesis
}

Jesus Ángel Pérez Fernández

Mechanical Engineer

TUTOR:

Enrique Alcalá Fazio

PhD Mechanical Engineer

Madrid 2017 



\section{ABSTRACT}

Heavy duty vehicles for passenger transport are mostly constituted by buses and coaches. The structures of these vehicles are subjected to variable loads over time during their operation, causing fatigue to be their main cause of failure, and thus the most restrictive criterion in their design. The great majority of these structures are composed of welded tubular profiles, so the inherent phenomenon of strain concentrations, associated with the weld bead created during this process, facilitates the crack initiation precisely in this zone. Therefore, an optimum design of these structures is directly linked to a precise fatigue analysis.

In order analyze the fatigue behavior of any component or system accurately, it is of vital importance to have a precise and representative characterization of the loads that it undergoes during its operation, as well as to know its resistant behavior against this phenomenon.

In this sense, the increasing development of finite element calculation techniques supposes an significant improvement in fatigue oriented design and analysis, since they allow to anticipate possible sources of failure in the initial design phases. The modeling of structures of large passenger transport vehicles is usually carried out in a simplified way by means of beam type elements, which allows to obtain satisfactory results of the overall behavior of the structure with a low computational cost. However, this type of model has certain limitations; among then the inability to reproduce the tensional behavior of the welded joints of the profiles, of vital importance in a fatigue analysis.

In this scenario, the main objective of the thesis is to develop an accurate fatigue life prediction methodology of these structures, capable to overcome the existing compromise between precision and complexity in the calculation.

To this end, a characterization of the dynamic loads of buses and coaches was carried out. The longitudinal and lateral accelerations were obtained by processing instant position data obtained with a GPS positioning system, installed in 10 different vehicles and recording more than 600 hours of operation. In the case of vertical accelerations, an accelerometer was used to capture the higher frequencies at which these events occur.

Additionally, a test plan was developed with the purpose of characterizing the structural and fatigue behavior of the welded joints that compose these structures. The studies carried out allowed to determine the resistant characteristics of the heat affected zone, obtain a strain to life curve of typical welded joints in buses and coaches, and also to characterize the strain concentration phenomena in these joints.

The process of characterization of strain concentrations in the vicinity of the welded joint was carried out by means of the Digital Image Correlation (DIC) technique, which allows obtaining a complete deformation field over the region of interest. This information was used for the construction and validation of a detailed finite element model capable of accurately 
reproducing the deformation distributions as well as the maximum deformations suffered by the joints, which is where the fatigue crack is expected to start. In spite of the high precision achieved in these detailed models, the construction of a complete structure with such models becomes practically unfeasible due to its complexity. In order to overcome this limitation, a series of regressive models were configured in order to establish a correlation between the maximum deformations obtained with these models and those obtained with simplified models using beam-type elements. This correlation was carried out on a total of 2304 different welded joints corresponding to different possible configurations present in structures of buses and coaches.

Finally, the results obtained in the different tests and calculations were structured to develop a routine for predicting the fatigue behavior of bus and coach structures modeled using beam type elements. This routine is able to unlink the compromise between precision and computational cost: on the one hand, it achieves high precision due to the application of realistic load conditions, a characterization of the fatigue behavior of the welded joints, and the correlation algorithm of maximum deformations between detailed and simplified models. On the other hand, it maintains a low computational cost due to the use of simplified models for the simulation of the response of the structures. 


\section{RESUMEN}

Los vehículos automóviles de grandes dimensiones destinados al transporte de pasajeros por carretera lo constituyen en su mayoría los autobuses y autocares. Las estructuras de estos vehículos están sometidas a cargas variables en el tiempo durante su funcionamiento, lo que provoca que la fatiga sea su principal causa de fallo, y por tanto el criterio más restrictivo en su diseño. La gran mayoría de estas estructuras están compuestas de perfiles tubulares soldados, por lo que el inherente fenómeno de concentración de deformaciones, asociado al cordón de soldadura creado durante este proceso, facilita el inicio de grieta precisamente en esta zona. Por ello, un diseño optimo de estas estructuras está directamente vinculado a un análisis de fatiga preciso.

Para poder analizar de forma fiable el comportamiento a fatiga de cualquier componente o sistema, es de vital importancia disponer de una caracterización precisa y representativa de las cargas que sufre durante su funcionamiento, así como conocer su comportamiento resistente frente a dicho fenómeno.

En este sentido, el desarrollo creciente de las técnicas de cálculo mediante elementos finitos suponen un avance importante en el diseño y calculo a fatiga, ya que permiten adelantarse a posibles fuentes de fallo en las fases iniciales de diseño. El modelado de estructuras de vehículos de grandes dimensiones de transporte de pasajeros se realiza habitualmente de forma simplificada mediante elementos tipo viga, que permiten obtener resultados satisfactorios del comportamiento global de la estructura con un bajo coste computacional. No obstante, este tipo de modelos presentan ciertas limitaciones; entre ellas la incapacidad de reproducir el comportamiento tensional de las uniones soldadas de los perfiles, de vital importancia en un análisis a fatiga.

Dentro de este escenario, el principal objetivo de esta tesis es el desarrollo de una metodología de predicción de vida a fatiga de estas estructuras precisa, capaz a su vez de salvar el compromiso existente entre precisión y complejidad en el cálculo.

Con este fin, se llevó a cabo en primer lugar una caracterización de las cargas dinámicas de funcionamiento de autobuses y autocares. Las aceleraciones longitudinales y laterales fueron obtenidas a partir del procesado de datos de posición obtenidos con un sistema de posicionamiento GPS, instalado en 10 vehículos diferentes y registrando más de 600 horas de funcionamiento. En el caso de las aceleraciones verticales, se utilizó un acelerómetro para poder captar las mayores frecuencias a las que ocurren estas solicitaciones.

Por otro lado, se desarrolló un plan de ensayos con la finalidad de caracterizar el comportamiento estructural y a fatiga de las uniones soldadas que componen estas estructuras. Estos estudios permitieron determinar las características resistentes del material afectado térmicamente por la soldadura, obtener una curva de deformación frente vida a fatiga de uniones soldadas típicas en autobuses y autocares, y caracterizar los fenómenos de concentración de deformaciones en estas uniones. 
El proceso de caracterización de deformaciones en las proximidades de la unión soldada se llevó a cabo mediante la técnica de correlación de imágenes digitales (del inglés, 'Digital Image Correlation', DIC), que permite la obtención de mapas de deformación completos sobre el área de enfoque de las cámaras utilizadas. Esta información fue utilizada para la construcción y validación de un modelo de elementos finitos capaz de reproducir de forma precisa estos mapas de deformación así como las máximas deformaciones sufridas por las uniones, que es donde se espera que aparezca la grieta de fatiga. Pese a la alta precisión conseguida en estos modelos de detalle, la construcción de una estructura completa con este tipo de modelos se hace prácticamente inviable debido a su complejidad. Para salvar esta limitación, se establecieron una serie de modelos regresivos para establecer una correlación entre las deformaciones máximas obtenidas con estos modelos, y las obtenidas con modelos simplificados que utilizan elementos tipo viga. Esta correlación se llevó a cabo sobre un total de 2304 uniones soldadas diferentes, correspondientes a diferentes posibles uniones presentes en estructuras de autobuses y autocares.

Finalmente, se estructuraron los resultados obtenidos en los diferentes ensayos y cálculos, para el desarrollo de una rutina capaz de predecir el comportamiento a fatiga de modelos de estructuras de autobuses y autocares modeladas mediante elementos tipo viga. Esta rutina logra desvincular el compromiso entre precisión y coste computacional: por un lado, consigue una elevada precisión debido a la aplicación de condiciones de carga realistas, a una caracterización del comportamiento a fatiga de las uniones soldadas, y al algoritmo de correlación de máximas deformaciones entre modelos de detalle y simplificados. Por otro lado, mantiene un bajo coste computacional debido a la utilización de modelos simplificados para la simulación de la respuesta de las estructuras. 


\section{ACKNOWLEDGEMENTS}

I would like to express my sincere gratitude to my supervisor Enrique Alcalá for all his time and effort and to Francisco Aparicio for giving me the chance to be part of the University Institute of Automobile Research (INSIA). I would like to thank my family, specially to my wife Carmen Escudero, for her constant support and understanding, and to my mother Isabel Fernandez, for making this opportunity happen. Without you, this thesis would have been impossible.

I also thank to Sam Coppieters and to the members of the Research Unit Mechanics of Materials, Products and Processes (KU Leuven, Belgium), for the opportunity given to perform a research project and a stay with them and for their support during this period.

I also would like to acknowledge the funding resources from the Spanish Ministry of Science and Innovation.

Finally, I gratefully acknowledge the whole crew of the University Institute of Automobile Research (INSIA) for their help during the development of the thesis. 


\section{CONTENTS}

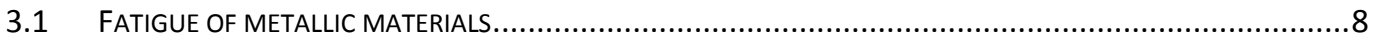

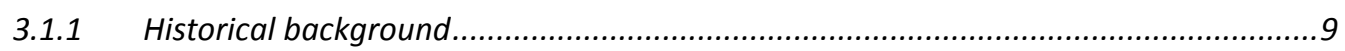

3.1.2 Theoretical basis of fatigue analysis..................................................................... 13

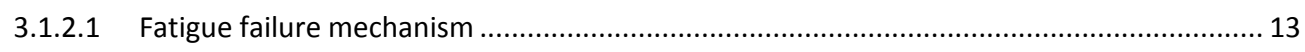

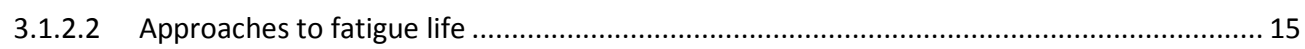

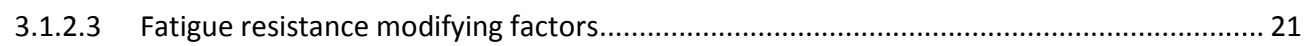

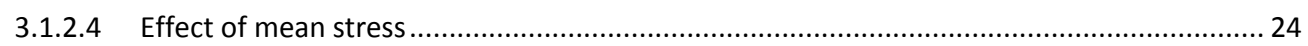

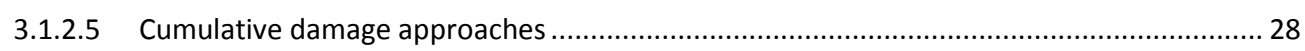

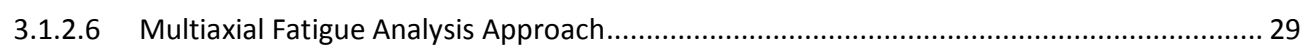

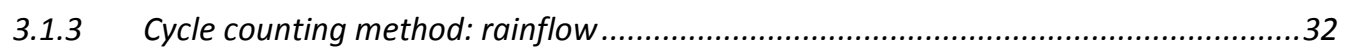

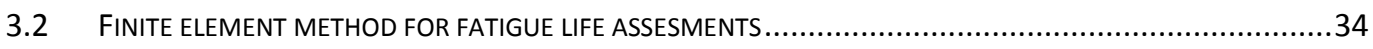

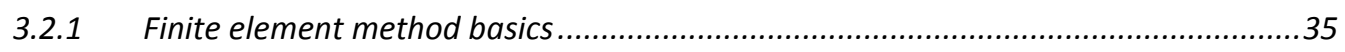

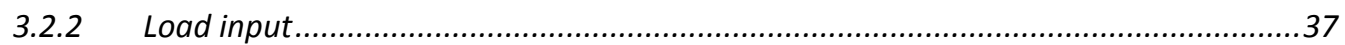

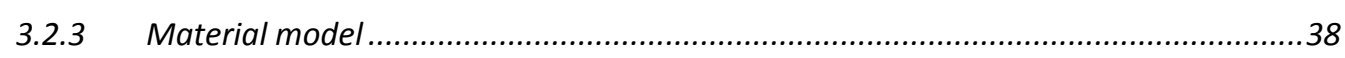

3.2.4 Calculating local strains in welded junctions .........................................................40

3.2.5 Review of bus body structures modelling techniques..............................................41

3.3 EXPERIMENTAL METHODS TO OBTAIN STRENGTH AND FATIGUE PROPERTIES ON WELDED JUNCTIONS ..........42

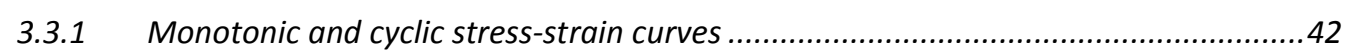

3.3.2 Determining Strain-Life curve from fatigue test data.............................................47

3.3.3 Strain concentration behavior on welded junctions ...............................................50

3.3.3.1 DIC for measuring strain concentrations in welded junctions........................................... 51

4 DYNAMIC LOAD CYCLES ON LPTV STRUCTURES DURING OPERATION .............................57

4.1 LATERAL AND LONGITUDINAL OPERATING LOADS FROM GPS POSITIONING DATA .................................57

4.2 VERTICAL OPERATING LOADS FROM ACCELEROMETER BASED ADQUISITION SYSTEM ...............................64

4.2.1 Evaluation of the accelerometer measuring quality...............................................65

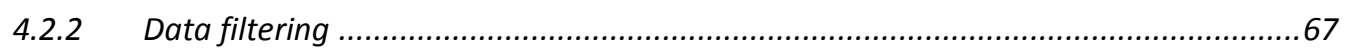

4.3 LOAD CYCLES: IMPLEMENTATION OF RAINFLOW CYCLE COUNTING METHOD OVER THE ACCELERATION TIME

5.1 STRESS-STRAIN CURVE OF WELDED STRUCTURAL STEEL .............................................................. 71

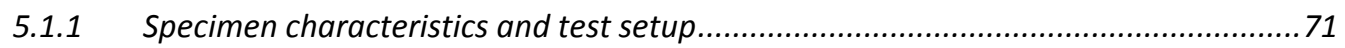

5.1.2 Test results: HAZ material properties .................................................................. 74

5.2 STRAIN CONCENTRATION BEHAVIOR OF RECTANGULAR TUBE BEAM WELDED JUNCTIONS .........................76

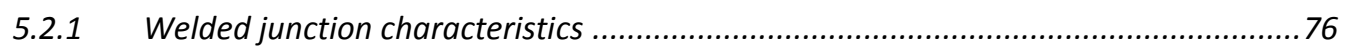

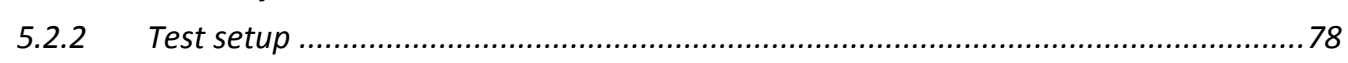

5.2.3 Image processing and smoothing error ............................................................... 83

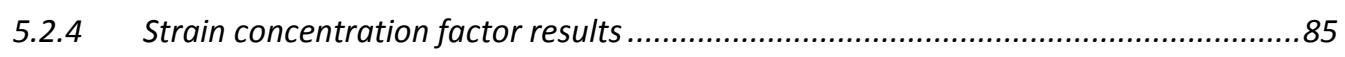

5.3 FATIGUE RESISTANCE OF RECTANGULAR TUBE BEAM WELDED JUNCTIONS: STRAIN-LIFE CURVE...................89 
5.3.1 Test configuration: obtention of maximum strain vs life data ..............................90

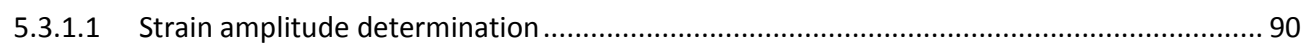

5.3.1.2 Obtaining the number cycles to failure ....................................................................... 91

5.3.2 Test results: strain-life curve parameters ..............................................................93

$6 \quad$ VALIDATION OF A FINITE ELEMENT JUNCTION MODEL ...............................................96

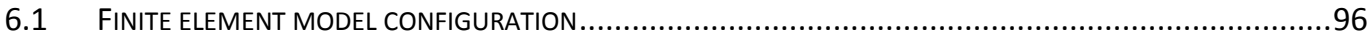

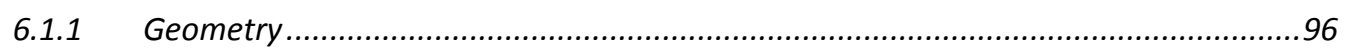

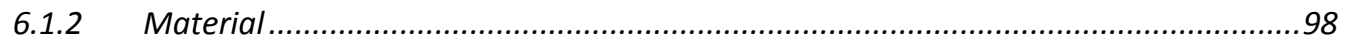

6.1.3 Meshing characteristics ............................................................................ 99

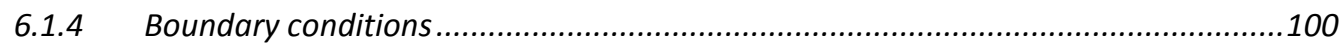

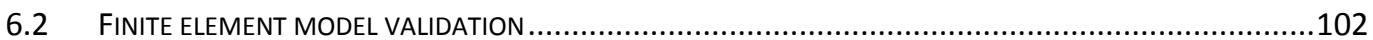

6.3 STRAIN STATE UNDER COMBINED LOADS: PROPORTIONALITY STUDY ............................................106

7 CORRELATION BETWEEN DETAILED AND SIMPLIFIED MODELS OF RECTANGULAR TUBE

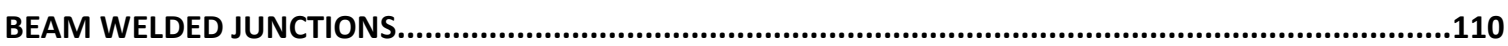

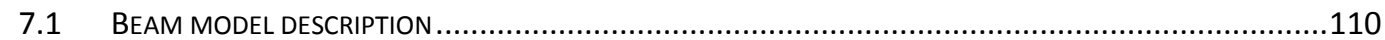

7.2 RECTANGULAR TUBE BEAM DIMENSION COMBINATIONS CALCULATED ............................................112

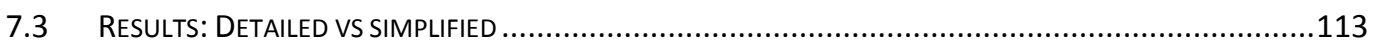

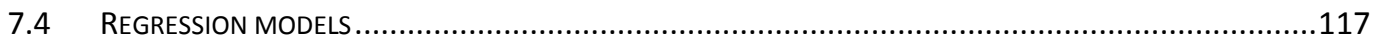

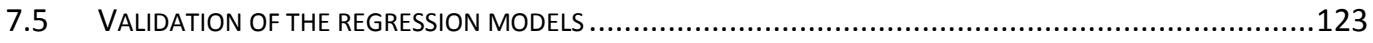

8 APPLICATION OF THE FATIGUE LIFE PREDICTION METODOLOGY TO A LPTV STRUCTURE: CASE STUDY 126

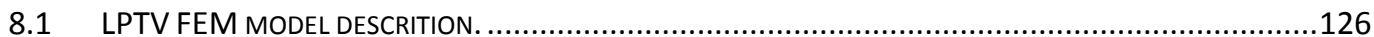

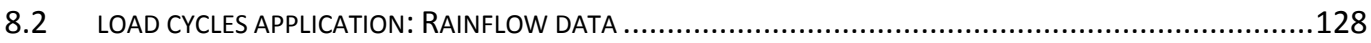

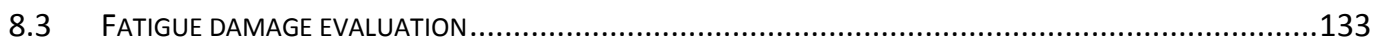

8.3.1 Maximum strains determination: regression models .............................................136

8.3.2 Determination of cycles to failure and fatigue damage ......................................138

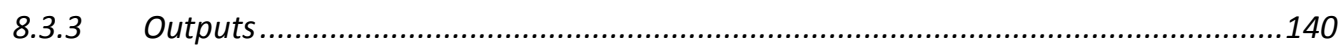

8.4 LOCATION OF DAMAGED JUNCTIONS IN THE STRUCTURE ........................................................... 143

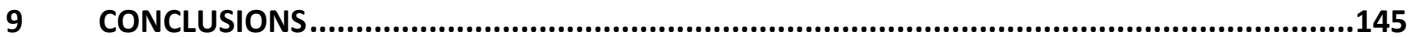

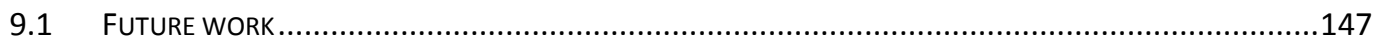

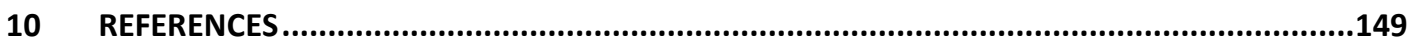





\section{INTRODUCTION}

Heavy duty vehicles for passenger transportation is currently one of the most important modes of transport nowadays. According to the Statistical Pocketbook published in 2016 by the European Commission, bus and coaches supposes $9 \%$ of the overall road transportation, and the total bus and coach fleet in Europe exceeds 800000 vehicles. Only in Spain, there are available a total of 60000 vehicles, which more than 1.6 billion passengers use every year, according to the 'Anuario Estadístico de España 2016' published by the 'Instituto Nacional de Estadistica'.

Automotive industry has become more and more demanding in the recent years, requiring lighter vehicles, more resistant, better equipped, and longer lasting, everything at a minimum cost. These framework compels the manufacturers within the sector to make important efforts on designing and fabricating optimized and efficient vehicles. Consequently, the investigations focused on the optimization of the vehicle design and its manufacturing process have increased notably in recent years.

In order to achieve an optimized design in structures and systems of vehicles, it is crucial to have a deep knowledge of the operating loads that they will withstand during normal functioning. Since vehicles are moving machines, these loads will vary with time, which converts fatigue in the most common cause of failure.

Until recently, design processes in the automotive industry relied in approximate calculations, based in manufacturer's experience and using rather basic design and calculation tools. This approach usually resulted on notably conservative solutions, and consequently, in not optimized results. In the last decades this methodology has been changing gradually, propelled by the increasingly restrictive environmental standards, and supported by the growing computer capabilities.

In this regard, calculations based on finite element methods have made a clear difference in the design process of complex structures and systems. This type of tools enable reproducing accurately the response of the structure in study, which permits addressing different design variables at the initial stages of a project, with the consequent advantages that is supposes from a technical and economical point of view.

With the support of these tools, a wide number of investigations have been carried out aimed to evaluate and improve bus and coach structural designs. The most extended practice is to implement the calculations over simplified models constructed with beam type elements (see the works presented by Lan et al., 2004; SJ Park \& W.S. Yoo, 2008; or R. Su et al., 2011; as an example). The huge increment of the computational capabilities experienced in the recent years has favored the use of more complex models that use combined shell or volume elements, with beam type ones (H. Hu et al., 2012; O. Ruiz et al., 2012).

Nevertheless, it is important to point that in order to achieve a simulation that reflects reality, not only the model geometry itself must be accurate, but also the material properties and the boundary conditions (i.e. input loads and constrains). In this regard, little is known 
about the real loads that a bus body withstands during normal operation, and the usual choice is to evaluate the designs under rather severe load cases (e.g. accelerations close to dynamic limits of the bus, high torsional states). Conservative load cases will lead inevitably to conservative designs independently from the accuracy of the model, which complicates the optimization of the structure.

The bus structures of large passenger transport vehicles (LPTV) are usually constructed by means of rectangular section beams joined by different welding processes. The welding bead resulting from this process generates local strain increments due to the localized stiffness changes generated. This behavior evidences another limitation of the modeling of bus structures using beam type elements: the inability of accounting for these local strain concentrations. Welded junctions modeled using beam type elements cannot account for the local stiffness changes on the junction area. On the other hand, even junctions modeled using shell or volume elements types, will lead to strain singularities in the junction corners unless the actual shape of the weld bead is also modeled.

In all, it is possible to point three main sources of uncertainty that need to be addressed for an optimized fatigue design of LPTV structures:

- Actual dynamic operating loads: in some machines or systems, operating loads can be easily estimated from the function for which it was designed. On the contrary, in most of the cases these loads are not know a priory, compelling to perform experimental procedures to acquire this data on similar products or prototypes.

- Fatigue behavior: the phenomenon of fatigue failure has an significant statistical dispersion, since it is highly dependent on local material imperfections and composition at a microscopic level. In addition, other variables like surface roughness, size, manufacturing process, etc. will have an impact on the fatigue resistance of the material, increasing the scatter of the results.

- Strain concentration effect: As already stated, the welded bead acts as a strain concentrator due to the local stiffness change that it causes. This strain concentration can change significantly from one junction to other due to the variability of the shape that the weld bead can adopt. Additionally, the measurement of these strain raisings becomes difficult due to the irregular shape that the weld bead presents.

The present thesis deeply approaches these three issues with the objective of developing a methodology that evaluates the fatigue behavior of LPTV structures with the maximum precision.

With the aim of characterizing the dynamic loads withstood by a LPTV during normal operation, two different acquisition equipments were employed. On the one hand, position data obtained from a Global Positioning System (GPS) were processed to determine lateral and longitudinal accelerations (J. Pérez et al., 2014). Second-order central differentiation method was used to obtain instant accelerations from the position data registered (A. Bruton et al.,1999). In all, more than 600 hours of operation were recorded over 10 different models of 
LPTV operating on 13 different urban routes. This proceeding was limited to lateral and longitudinal accelerations, since vertical ones usually arise from road imperfections that produce higher frequency accelerations, difficult to detect by a GPS equipment. For this reason, it was decided to build a standalone acquisition equipment based on a triaxial accelerometer and a storage system, capable of acquiring acceleration data at the sufficient rate. It was installed onboard of 6 different EMT routes, at different times of the day during approximately 8 hours.

After obtained all acceleration data, fatigue cycles were extracted using the cycle counting method "Rainflow" (C. McInnes and P. Meehan, 2008). These fatigue cycles were afterwards extrapolated to the lifespan of the vehicle to obtain the overall acceleration cycles that will withstand in the entire life.

As stated above, the behavior of materials under fatigue loads is significantly scatter itself. In addition, fatigue experiments are costly both in time, since these tests can take days or even weeks depending on the loading level and frequency, and in means, since they usually require specific equipments. For these reasons, the most common practice is to estimate the fatigue behavior of a component from fatigue data of the material (tested in laboratories, in very controlled loads and specimen characteristics), and later extrapolate this information to the actual component utilizing a series of modifying factors. This proceeding is well accepted and extended in the industry, since it provides a simple and timeless approach to the fatigue strength of a component. Nevertheless, it is important to note that the scatter of this method is significantly high, since the sources of uncertainty arise not only from the fatigue phenomenon itself, but also from the application of the modifying factors, that will adapt with limited precision to the specific characteristics of the actual component.

Although more costly, the most precise manner to characterize the fatigue behavior of a component, is to test the actual component under the loads that with suffer during the lifespan. With this aim, over 30 T-type welded junctions, typically present on LPTV structures, were constructed and tested under different levels of cyclic loading. Bending load type was applied since it was found to be predominant on LPTV structures during normal operation. An Instron ${ }^{\circledR}$ hydraulic actuator was used to apply controlled loads and register the number of cycles to failure. On the other hand, a Digital Image Correlation (DIC) equipment was utilized to measure the maximum strains suffered by the junction for the different load levels. In this way, a point in the strain-life $(\varepsilon-\mathrm{N})$ curve was obtained for each junction tested. Once finished all experiments, the actual $\varepsilon-\mathrm{N}$ of the component can be determined by means of by data regression.

Using the capabilities of DIC technology, shape, displacement and strain fields were obtained close to strain concentration area (i.e. the weld bead toe) for 10 different welded junctions subjected to bending loads in two orthogonal directions. In this manner we mimic the lateral and longitudinal bending moments that these junctions should withstand on a LPTV structure. By the later processing of this information, it was carried out a complete characterization of the strain concentration behavior of the welded junctions of rectangular tube beams. 
In addition, the obtained data with the DIC experiments was employed to construct and validate a detailed finite element model which reflects the welded junction geometrical characteristics. These geometrical characteristics were in turn determined from the DIC shape fields obtained from the measured junctions. A validation metric based on confidence intervals was later employed to assess the precision of the FEM model.

Once having a validated junction model, equivalent models were constructed for different profile combinations that can be present on actual LPTV structures. In all, 2304 different models were constructed using different combinations of the profile section geometry for each junction type. From the evaluation of these data, significant characteristics of the strain concentration behavior in relation to the geometrical variables were obtained.

In parallel, the same models were calculated using the simplified modeling technique based on beam type elements, usually employed when calculating complete LPTV structures. Peak strain values were later evaluated in order to obtain a regression algorithm that creates a relation between the values obtained from simplified and detailed models.

The regression models thus obtained permits an accurate estimation of the maximum strains suffered by a LPTV junction simulated with simplified models, thus supposing a significant accuracy improvement with almost no computational cost increase.

All experiments and validations described previously were finally structured to develop the methodology presented in the schema of Figure 1. As already stated, this methodology aims to evaluate the fatigue behavior of welded junctions of LPTV structures from simplified FEM models using beam type elements.

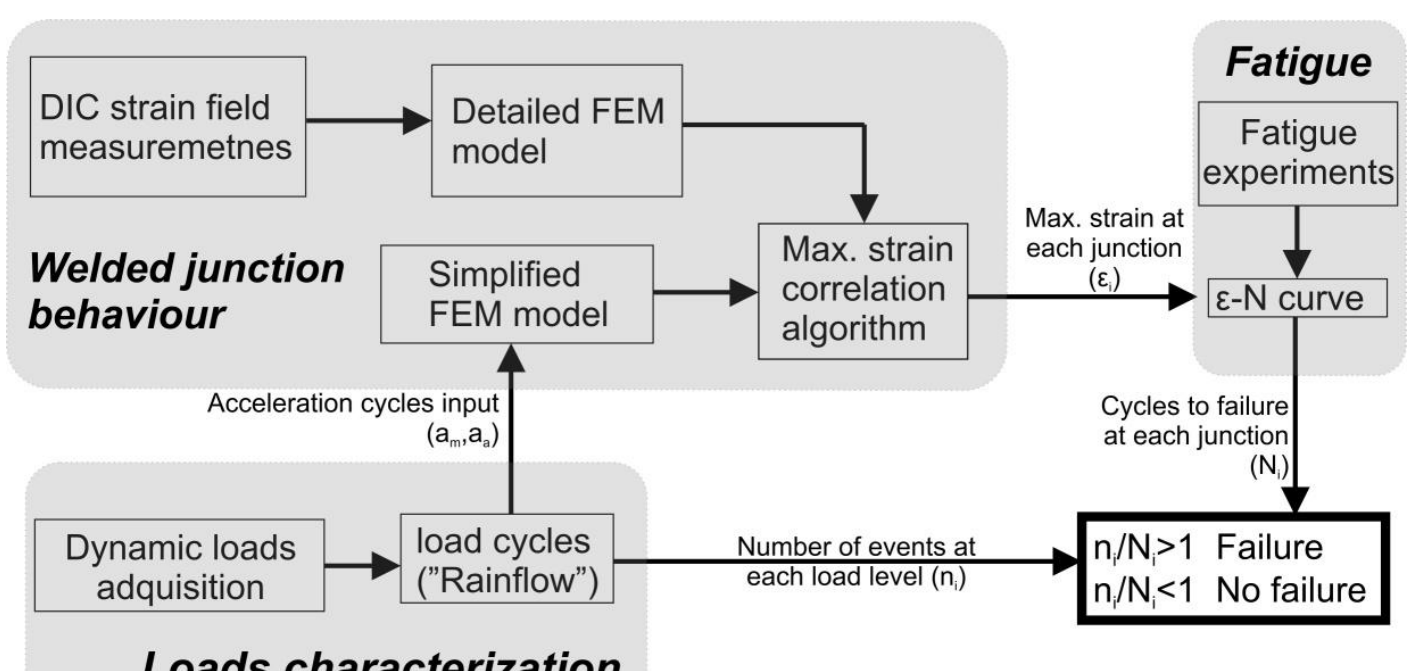

\section{Loads characterization}

Figure 1 Fatigue evaluation methodology schema

Three main blocks can be distinguished in the schema, each of them approaching to one of the three uncertainty sources when characterizing fatigue behavior described above. Concerning the dynamic loads during operation, the mentioned steps for instrumentation and acquisition of the accelerations was first carried out. Later, the data thus obtained was processed to extract the acceleration cycles using the 'Rainflow' cycle counting method. These 
acceleration cycles, organized in classes according to different coupled levels of mean and alternate acceleration $\left(a_{m}, a_{a}\right)$, are the input of the simplified FEM model of LPTV structure in study.

Regarding the block of actions aimed to study the strain concentration behavior of the junction, different welded profiles were tested in to two different bending load directions in order to characterize statistically both the SCF and the geometrical characteristics of the welded bead. These characteristics were employed to construct and validate a detailed FEM model of the junction that reproduces accurately its strain concentration phenomenon. After this, a correlation algorithm that relates the maximum strains obtained with detailed and simplified models was developed for different possible welded junctions present in LPTV structures. This algorithm has the function in the methodology of correcting the strain values obtained in the calculation of the complete LPTV structure model with the acceleration cycles.

The corrected strain values obtained are the input values of the e- $\mathrm{N}$ curve of the junction. As stated before, this curve was obtained by means of specific fatigue testing in order to avoid further scattering sources. As a result, the number of cycles that each junction of the structure can withstand before failure are obtained.

Additionally, during the acceleration acquisition process, the number of events for each $\left(a_{m}, a_{a}\right)$ class was determined. Due to the representativeness of the data obtained, this number of events can be extrapolated to the desired lifespan of the vehicle (i.e. amortization period).

Finally, through the comparison of the number of cycles that the junction can support before failure, with the number of events to which it will be subjected during the lifespan, it can be quantified the 'damage' of each junction using the Palmgren-Miner rule (A.Fatemi and L.Yang, 1998). Depending on the damage value obtained, three different scenarios can be distinguished:

- Damage >1: The junction will present failure before the lifespan of the vehicle finishes. The higher the damage, the sooner the junction will fail.

- Damage between 0 and 1: The junction is expected to present failure, although after the lifespan if the vehicle.

- Damage $=0$ : The junction presents infinite life (i.e. strains suffered are always under the fatigue limit).

The thesis has been structured in different sections related to the methodology presented, each approaching a different subject area as described below.

After the introduction to the problematic and scope of the thesis presented in the current Section 1, the following Section 2 summarizes its main objectives.

Section 3 gathers the state of the art and the theoretical background of the different topics approached in the thesis. The chapter is in turn subdivided according to the different subject areas, covering concepts related to fatigue behavior of metallic materials, finite element 
calculation and the experimental methodologies to characterize the strength and fatigue properties of metallic materials.

In Section 4, the process followed for the characterization of the dynamics loads withstood by the LPTV structures during normal operation is described. First, the methodologies used to register the acceleration data needed are presented, and later, the cycles obtained by means of the cycle counting method 'Rainflow' are shown.

The different tests focused on studying the structural behavior of the LPTV welded junctions are presented in Section 5. This chapter covers the strength and fatigue resistance of the junctions, as well as the characterization of the strain concentration phenomenon.

The construction and validation of the detailed FEM model of the welded junction from the information obtained previously is described in Section 6 . It details the model construction process and shows the results of the validation metric applied using the experimental data.

Section 7 presents the correlation algorithm developed to relate the maximum strains calculated by means of detailed and simplified junction models. Also within this chapter, the influence of the dimensions of the rectangular tube beam sections on the maximum strains is also evaluated.

The application of the complete methodology is shown in Section 8 by means of a practical case study that evaluates the fatigue behavior on a complete LPTV structure. A possible routine for an automated evaluation of the structure is also brought in this section.

Finally, in Section 9, the conclusions, contributions and future work of the thesis are presented. 


\section{OBJECTIVES}

The main goal of the present thesis is to develop a methodology that evaluates the fatigue resistance of the LPTV welded structures, from FEM models constructed using beam type elements. The methodology aims to decouple the high precision model needed to account for the local strain raisings at the junction, from the computational cost that would suppose to model a whole LPTV structure in such level of detail.

For the achievement of the main goal, the following sub-objectives must be covered with the maximum precision:

- A precise and representative characterization of the dynamic loads that the LPTV will withstand during normal operation. As already stated, a FEM model will not be realistic if the input loads do not reflect reality, regardless its accuracy.

- Characterize the strain concentration phenomenon on this type of welded junctions in order to establish a validated correlation between the actual maximum strains and the beam type simulation model results.

- Obtain a specific strain to life curve of the junctions. This avoids further uncertainty sources arising from the use of material fatigue data and its corresponding correcting factors. 


\section{STATE OF THE ART}

Fatigue of structures is directly related to loads that vary during the operation time. The first structures constructed based on metallic materials were usually subjected to static loads, with little or no variable component. Therefore, fatigue was a hardly known phenomenon until ninetieth century, when some systematic failures were observed in railway structures subjected to stresses well below the material strength limits. Due to the catastrophic consequences in terms of safety and cost that these failures usually entailed, increasing efforts were brought together in order to better understand and thus prevent them. From this time, the investigations related to fatigue phenomenon have continuously increased, supplying concepts and valuable know-how of great use nowadays. Despite the important improvements achieved during all this period, fatigue is still the most common cause of mechanical failure on components or systems, and has a specially decisive influence in motion related industries like automotive, railway, aeronautics or naval. Therefore, fatigue phenomenon has been linked to automotive industry from the very beginning, due to the inherent relation between mobility and time varying loads. In this regard, the main concerns that have enhanced fatigue investigations are economical, safety, and in the last years environmental.

The increasing computer capabilities experienced in the last years has made possible the development of fatigue assessment techniques based on complex structural calculations. Within this field, most of the progress has been carried out with the aid of the finite element method.

In the following sections, the studies and concepts utilized during the development of the methodology of the present thesis will be introduced.

\subsection{FATIGUE OF METALLIC MATERIALS}

Fatigue (from the Latin word 'fatigare' which means 'to tire') can be understood as the premature failure of a material when is subjected to time varying stresses well below the strength limits or even the yield point. Its failure mode consists of the generation and propagation of a crack that eventually provokes the breakage of the component or structure. In metallic materials, this crack is locally generated due to sliding of the crystalline structure planes consequence of shear stresses.

During operation, any component is subjected to loads that vary over time. Nevertheless, in many occasions the variable component is considered negligible and the analysis is performed supposing constant loads. In any case, an evaluation similar to the one shown in Figure 2 is recommended in order to assess whether the component should be analyzed to fatigue. The initial input information are the operating loads, or an estimation of them, and the design of the component. With this information it is possible to calculate or measure the response of the component when loaded. If it is very close to constant, the stresses and strains will be also constant and thus a static analysis can be performed. Conversely, if the component response is time varying, it would be necessary a deeper study on the stress and strains cycles suffered $\left(\sigma_{m}, \sigma_{a}\right)$ with the aid of some cycle counting technique (Y.L. et al., 2005). In the case that these 
loads are below the fatigue limit of the component after applying the corresponding safety factors (SF), the strength analysis can be simplified to static. On the contrary, if the stress or strain cycles are found to be above the component fatigue limit, it will be necessary to perform a fatigue analysis.

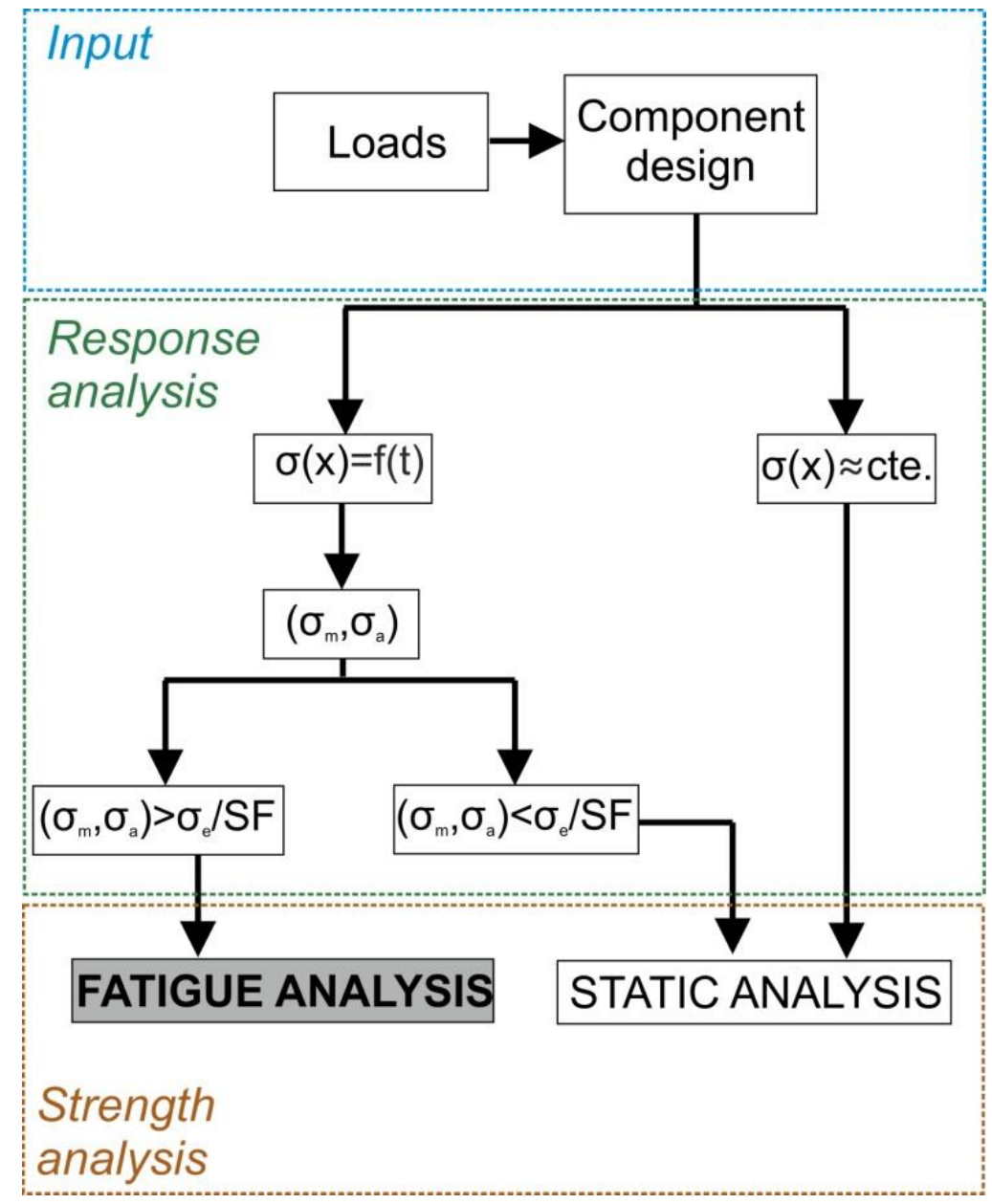

Figure 2 Strength analysis decision schema.

From what was observed in the schema, the link between fatigue and automotive industry seems to be unavoidable. Automobiles are machines whose main purpose is transportation and, as such, their components will suffer significant varying loads during operation. Consequently, fatigue analysis will be needed for most vehicles components. LPTV structures is obviously not an exception.

In the following sections, a revision of the state of the knowledge of the different fatigue analysis concepts and criterions considered relevant for the current thesis will be presented.

\subsubsection{HISTORICAL BACKGROUND}

It has been known for centuries that some materials such as metals or timber could present failure when they are subjected to a few high amplitude load cycles, i.e. above the yield point. Nevertheless, until the development of steel machinery and railway transport industry in the early ninetieth century it was unknown that load cycles under yield limit could also cause material failure. 
The first bibliographic reference to the fatigue phenomenon dates from 1837 (W.A.J. Albert); it refers to what is believed to be the first investigation related to fatigue, carried out by the German mining engineer W.A.J. Albert around 1829, consisting of repeated cyclic load proof tests on mine hoist chains made of iron. Shortly after, W.J.M. Rankine in 1842, (better known from the Rankine thermodynamic process) analyzed and discussed possible causes of breakages in railway axles. He showed the durability improvement when reducing the notch effect in these parts. The term 'fatigue' is believed to be used for the first time by F. Braithwaite in 1854, he described fatigue failure on many components like water pumps, crankshafts, railway axles, etc., and also introduced the idea of an allowable stress for fatigue loaded components.

One of the greatest contributions to fatigue knowledge is the work developed by A. Wöhler between 1852 and 1870 . He configured and conducted the first systematic investigation on fatigue of materials. For that, he designed, among others, the so-called 'rotating bending test machine', which basic concept is still used nowadays. The schema of the machine is shown in Figure 3, it consists of a round specimen that rotates from the action of a motor at the same time that is subjected to a constant bending load. As the specimen spins, a point on the surface of the specimen will suffer tension compression cycles as it changes the position. With this testing methodology, Wöhler tested a significant amount of railroad axles, obtaining very valuable results of the fatigue behavior of these components. Some of the most important fatigue related concepts introduced by Wöhler to the research community are the relation between service loads and life, the design for finite life, the crack propagation or the quantitative information of the fatigue life decrease in relation to the notch effect. Nowadays, the curves that represent the relation between stress amplitude and cycles to failure in the $\sigma-\mathrm{N}$ diagram are known as Wöhler curves. Nevertheless, Wöhler always presented his test results in form of tables, it was actually his successor L. Spangenberg who first plotted them as curves in 1936. Many of the results reported by Wöhler were early after confirmed by Bauschinger (1886), who also introduced the idea that the elastic limit of a material subjected to cyclic loads can differ from the one observed under monotonic loading.

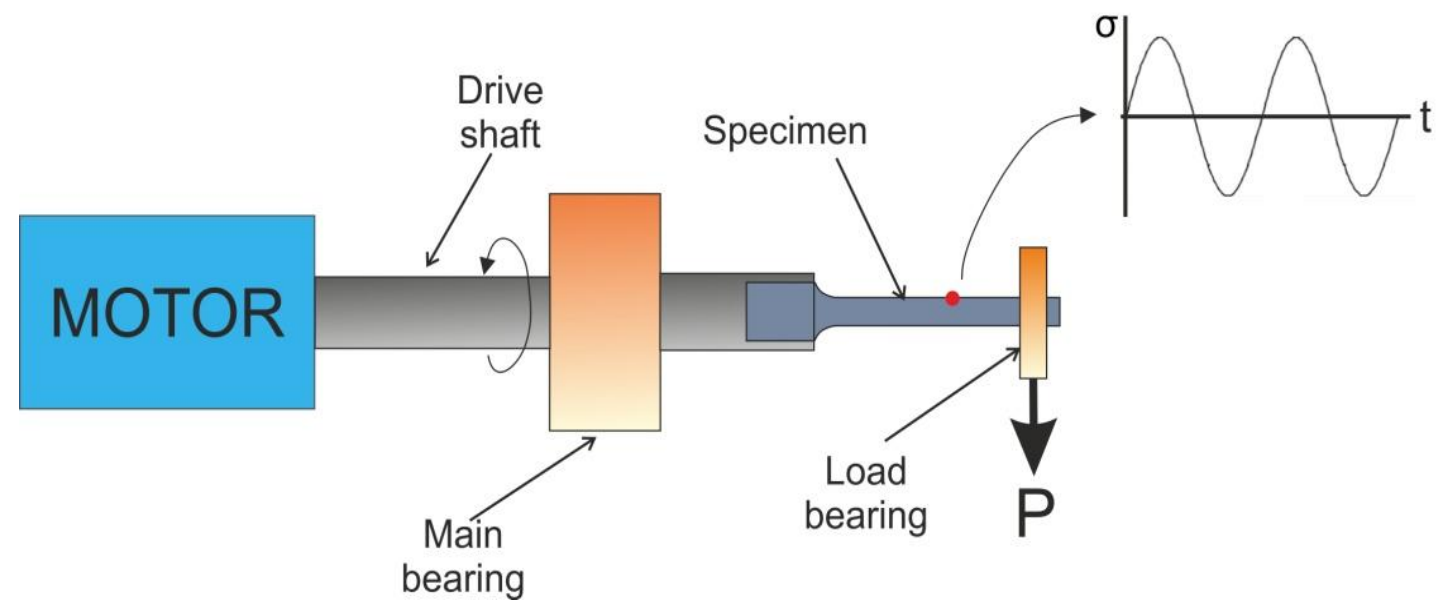

Figure 3 Wöhler rotating bending testing machine schema

H. Gerber proposed little after the publication of Wöhler work an analytic correction of the fatigue life when the mean stresses are nonzero. This research field was completed by the 
alternative correction expressions proposed by J. Goodman (1899) and C.R. Soderberg (1930). A fair comparison of these three approaches and its applications for steel alloys can be found in the work of Q. Bader and E. Kadum (2014).

Simultaneously, different researchers started to focus their research on failures of bridge structures due to alternating loads. By the beginning of the XX century there were more than 80 published manuscripts related to fatigue. In 1903, Ewing and Humphrey provided the first metallurgical description of the fatigue process after observing the slip bands that appear when loads over the proportionality limit are applied. The North American O.H. Basquin proposed an empirical equation (1.1) that related the stress amplitude $\left(\sigma_{a}\right)$ against the cycles to failure $\left(N_{f}\right)$ for the finite life proposed by Wöhler. This equation is still in use nowadays.

$$
\sigma_{a}=A\left(N_{f}\right)^{B}
$$

Between 1920 and 1940 it was coined most of the knowledge of fatigue of materials that is enjoyed nowadays. This period of research was led by the German community. Proof of it can be found in the book 'The Relation of Fatigue to Modern Engine Design' (1935), that gathers the most important contributions published between 1927 and 1935, and more than half of the references are German. Within this period it should be noted the contribution to the concept of 'cumulative damage' brought by A. Palmgren. This concept remained completely unknown until 1945 when Miner presented independently the same damage-accumulation hypothesis. It is currently the most employed rule to characterize cumulative damage, and is known as the Palmgren-Miner rule. Palmgren also developed for the first time a numerical approach to the probability of survival for fatigue loaded components. It is also remarkable the contribution to the statistical aspect of fatigue provided by W. Weibull (1939). He developed a probabilistic distribution to predict the fatigue failure probability baptized with his name, which later validated (1955) by means of thousand of tests on aluminum specimens and threaded blots.

During the 40's and 50's, most of the investigations carried out in the previous decades began to find industrial applications. Automotive and aircraft industries grew notably during these decades, accompanied by a consequent increase of catastrophic accidents, many of which with causes related to fatigue failure. Well-known examples are found in the two crashes documented in less than one year of the aircraft "Comet" due to structural fatigue failure, or the two Boeing B-47 nuclear bombers that crashed due to fatigue failure of the wing in 1958. Besides the B-47, the United States Air Force (USAF) suffered other fatigue related breakages in its warplanes that harmed the national safety of the US. Consequence of the numerous accident registered, two new terms, clearly focused on the industry, were brought into discussion: 'safe life' and 'fail safe'. The term 'safe life' states that a component must last the entire lifespan to which it was designed before experienced failure. 'Fail safe' refers to the fact that a component failure must not compromise the integrity of the structure. From these decades it is remarkable the contributions made by Coffin and Manson, who during 1954 published independently an analytic expression to relate the load reversals to the plastic strains. This expression is nowadays known as the Coffin-Manson rule. It should be also remarked during this period the complete 
handbook of stress concentration factors for different notch geometries published by Peterson in 1953.

During the posterior years, the investigations related to fatigue increased significantly and diversified in different sub-fields. One of the most deeply approaches were the investigations related to the characterization of the fatigue failure by means of crack propagation models. Pioneer advance in this field was brought by Irwin, who showed that the stress singularity present ahead of a crack can be expressed as a scalar factor, so-called the stress intensity factor ' $K$ '. Based on the Linear Elastic Fracture Mechanics (LEFM) principles, when ' $K$ ' reaches a critical value, the material will experience instant fracture. Paris et al. (1961), established shortly after that the fatigue crack propagation can be expressed by an analytical expression that directly depends on the stress intensity factor range ' $\Delta K$ '. This approach was soon very well accepted (even erroneously baptized as rule), and is still one of the most used expressions for characterizing the growth of the fatigue crack.

From 1960 onward the rapid development of the linear fracture mechanics concepts caused that the number of investigations and thus the publications related to fatigue issues increased still further. In the past four decades, important advances have been achieved in the understanding of cyclic deformation and crack initiation and growth mechanisms in fatigue. An important contribution is found in the work of Elber, who demonstrated in his PhD Thesis in 1968 that after a high tensile load the crack closes before the load is reduced to zero. Showing that a crack can therefore remain closed even when subjected to cyclic tensile loads due to the plastic deformations at the crack end. It should be also mentioned the USAF, which introduced the concept of 'Damage Tolerance Requirements' (1979), where crack-like defects are assumed to exist in all critical points of the structure caused by the manufacturing process or the operating loads. From their side, the manufacturers had to demonstrate that the structure will show sufficient life in these cracked conditions.

In the last decades, the technologic improvements of the testing equipments made possible experiments in more complex systems and load conditions; also the large amount of available data has allowed to develop life prediction models that have shown to fit the reality with fair accuracy. The researches and publications related to fatigue analysis has become more practical and specific in the recent years, but the rate of new publications have not ceased. In any case, some contributions should be highlighted in this text due to its close relation to the field of the thesis. The International Institute of Welding (Fricke, 2010), brings useful guidelines when modeling welded junctions by means of finite element method. Also, S.J. Maddox (1992) presents a complete asset of rules for designing welded structures subjected to fatigue loads. D. Radaj (2006) has also made notable contributions to assess the fatigue behavior of welded joints. More generally, the International Conferences of Fatigue Damage of Structural Materials and of Integrity-Reliability-Failure are worth to mention for gathering notable investigation groups every edition. 
Despite all the efforts, the knowledge of the fatigue behavior of materials is still incomplete, and the development of reliable life prediction models still involves constructing specific empirical models based on test results.

\subsubsection{THEORETICAL BASIS OF FATIGUE ANALYSIS}

There can be distinguished three approaches to fatigue phenomenon: scientific, material engineering and mechanical engineering. In global terms, the scientific approach focuses on the causes and effect of fatigue at an atomic scale, where dimensions are measured on Angstrom or nanometers. Materials engineering studies the microscopic crystalline structure of the materials and its evolution during fatigue development. Finally, the goal of the mechanical engineering approach to fatigue is the design and manufacturing of effective products, characterizing the fatigue behavior of materials at a macroscopic level. The scope of the work developed in the current thesis is clearly oriented to the mechanics engineering point of view of fatigue, and therefore the theoretical basis presented in the next sections will focus on this approach.

\subsubsection{Fatigue failure mechanism}

As previously stated, fatigue loads will cause any material to failure at stress values much lower than the maximum strength characteristics in monotonic loading conditions. The fatigue damage is a localized and cumulative process consisting of three main differenced stages: crack initiation or nucleation, crack propagation and final fracture. A typical fatigue fracture surface of a shaft can be observed in Figure 4, where these stages are observed.

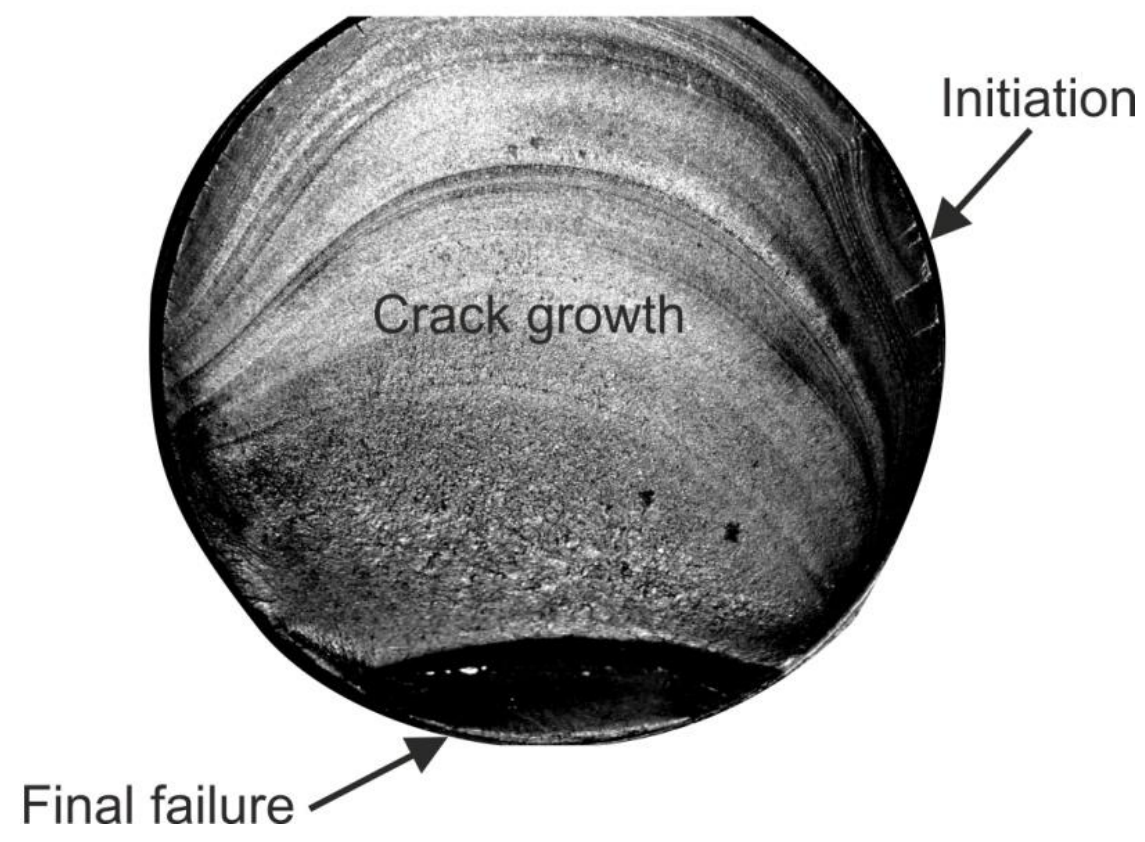

Figure 4 Fatigue fracture surface.

The initial phase of the fatigue damage corresponds to the crack initiation or nucleation. This stage strongly depends on the macro and micro structure of the material as well as on the load conditions. Most of fatigue cracks initiate at visible material discontinuities such as drills, 
slots, welds, keyways, etc., which act as stress multipliers that eventually causes local plastic flow. This irreversible deformation provokes the persistent slip of crystalline bands that act, in turn, as a micro stress multipliers (Figure 5). New load cycles will add more deformation energy, causing new dislocations that will eventually combine causing a dominant crack. This crack propagates on the local maximum shear stress plane (i.e. 45으 from the normal stress direction), and usually advances until any microscopic discontinuity such as grain boundaries, pores, inclusions, etc. For this reason, steels with small grain structures tend to have a better fatigue behavior. The initiation stage can last many thousands of fatigue cycles, or even stop the propagation at a grain boundary if the load applied is sufficiently low.

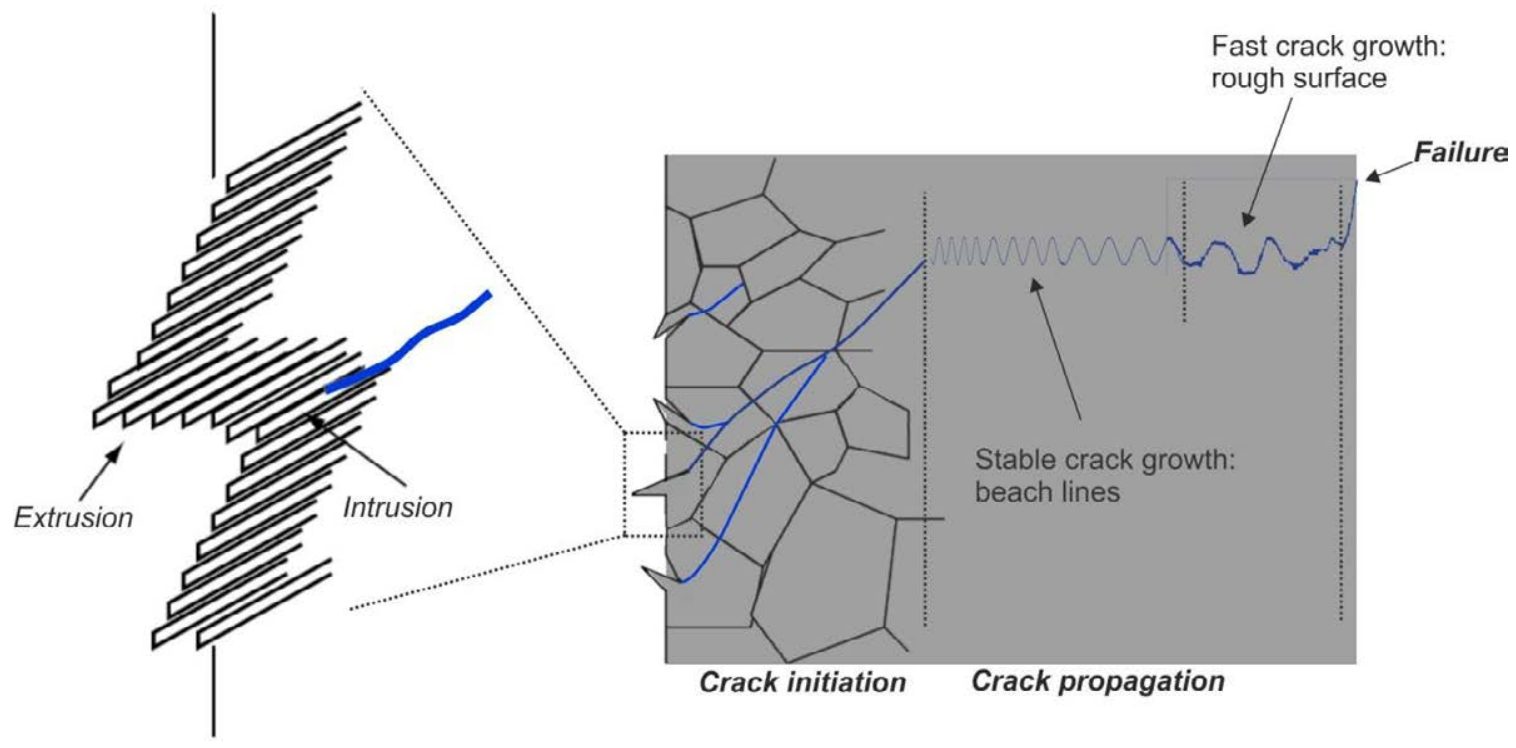

Figure 5 Fatigue crack evolution.

Once the crack has a relative significant size of several grains the second stage of the fatigue crack starts. At this stage, the advance direction changes and becomes perpendicular to normal load direction. This is the reason why ductile materials fractured by fatigue loads show brittle breakage.

At this point, the crack itself supposes a very high stress concentration source, so it propagates in a stable manner every cycle. The crack growth rate increases with the number of fatigue cycles applied, since the net resistant section to withstand the load becomes smaller, increasing the existing stresses. The crack growth rate also depends on the material characteristics: as a rule of thumb, cracks propagate faster on harder materials (i.e. high strength steels). It is possible to observe microscopic striations consequence of the cyclic advance of the crack during this stage; whose distance increases as the crack grows faster. Sometimes it is possible to distinguish macroscopic marks so-called 'beach lines' (Figure 4) formed by hundreds or even thousands of these striations.

As the crack advances and the resistant section decreases, the mentioned 'beach lines' disappear and the surface acquires an rough aspect similar to the one found in brittle breakages. 
El aspect of this surface is consequence of the static failure modes present at such elevated stresses at the crack tip, and the lack of friction between faces due to the rapid crack growth.

Eventually, the crack would have progressed at a point that the remaining resistant section would be too small to withstand on single load cycle, and the fatigue failure will occur. The lower the fatigue loads are, the smaller the section corresponding to this final failure will be.

\subsubsection{Approaches to fatigue life}

Due to the nature of fatigue phenomenon, the material failure is usually difficult to observe in advance, causing unexpected breakages in components where fatigue resistance has not been taken into account in the design process. These failures have a special catastrophic component in industries like railway, automotive or aircraft, where in many occasions involves human fatalities, or important material damages. This scenario has propelled important research efforts aimed to characterize the life expected by a material with respect to its load level, in order to predict and thus prevent/manage the fatigue failure with a certain confidence level.

There are three principal approaches to estimate fatigue life that will be presented in the next sub-sections: Stress-life, Strain-life and Linear Fracture Mechanics. There is not a clear rule to ensure the best choice for all situations, and the choice must be made attending to variables like material, load history, environment, geometry, or consequences of failure.

Currently, none of these methods are accurate enough to eliminate the need for components testing. In addition, the precision of them is a function of how accurate the input variables are defined.

\subsection{Stress-Life Based Approach}

The stress to life $(\sigma-N)$ approach was the first attempt to understand and quantify the fatigue of materials, developed by Wöhler more than 150 years ago. The fact that is the most simple approach, along with the large amount of data available of material fatigue properties using this method, makes it one of the most used approaches in the industry still today. The approach is best suited for applications where the stress levels are within the elastic range of the material, that is, what is known as the 'high cycle fatigue' (HCF).

The basis of this approach is the characterization of the material fatigue life versus the stress level, represented through the Wöhler curve in the $\sigma-\mathrm{N}$ diagram, which is usually presented on a log-log scale. Each point of this diagram corresponds to a different fatigue test. The most common testing procedure is the rotating bending test presented in Figure 3.

Figure 6 shows the typical aspect of the Wöhler curve for steels on the log-log scale, where three different slopes can be distinguished. The first slope change corresponds to the transition from the so-called 'low cycle fatigue '(LCF) to the 'high cycle fatigue' (HCF). The $\sigma-\mathrm{N}$ approach is not suitable for a fatigue life under this point. It can also be observed that for a sufficiently low stress value, the curve becomes horizontal. This stress value is known as the fatigue limit $\left(\sigma_{\mathrm{e}}\right)$, and a material subjected to stress cycles under this limit will not show failure. 


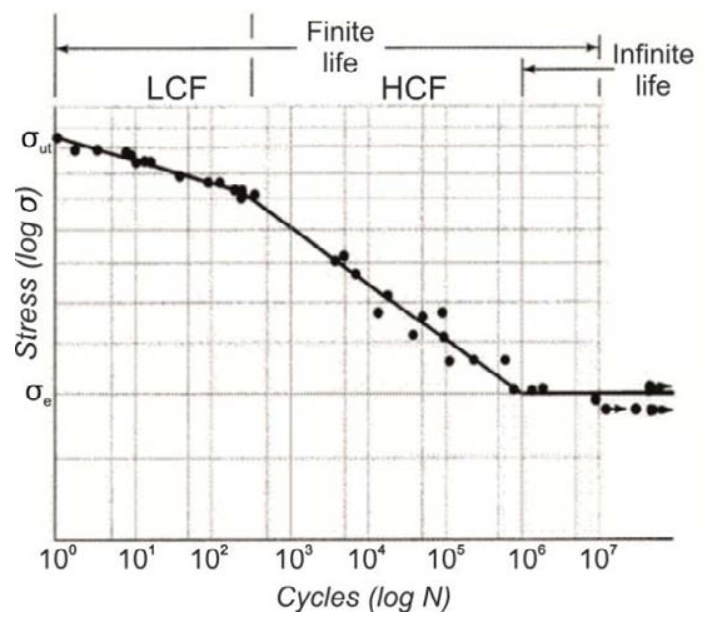

Figure 6 Stress life diagram and Wöhler curve.

Basquin observed that the part of the curve of the HCF can be accurately defined by a straight line in the logarithmic scale, which is in turn a power law function in the linear scale written as:

$$
\sigma_{a}=\sigma_{f}^{\prime}\left(N_{f}\right)^{b}
$$

Where:

- $\sigma_{\mathrm{a}}$ : is the fatigue stress amplitude.

- $\mathrm{N}_{\mathrm{f}}$ : is the number of cycles to failure.

- $\sigma_{f}^{\prime}$ : is the fatigue strength coefficient.

- $b$ : is the fatigue strength exponent.

According to this expression, only two fatigue test would be sufficient to determine the parameter ' $\sigma_{a}$ ' and ' $b$ '. Nevertheless, the scatter nature of fatigue compels to perform further experiments to obtain reliable results. In general terms the recommended number of test samples (ASTM E739-10) used to generate the $\mathrm{S}-\mathrm{N}$ curve is:

- 6-12 for preliminary and research and development tests.

- 12-24 for design allowable and reliability tests

\subsection{Strain-Life Based Approach}

The strain life based approach, also known as the local stress-strain or critical location approach, deals with the fatigue behavior of the material based on its total strain. Although most fatigue cases are related to elastic nominal stresses, the presence of notches, welds, or other stress concentrations in the component can result in local plastics deformation. In these conditions, a fatigue load cycle will entail a strain at the notch point composed by both elastic and plastic components, forming the hysteresis loop shown in Figure 7. 


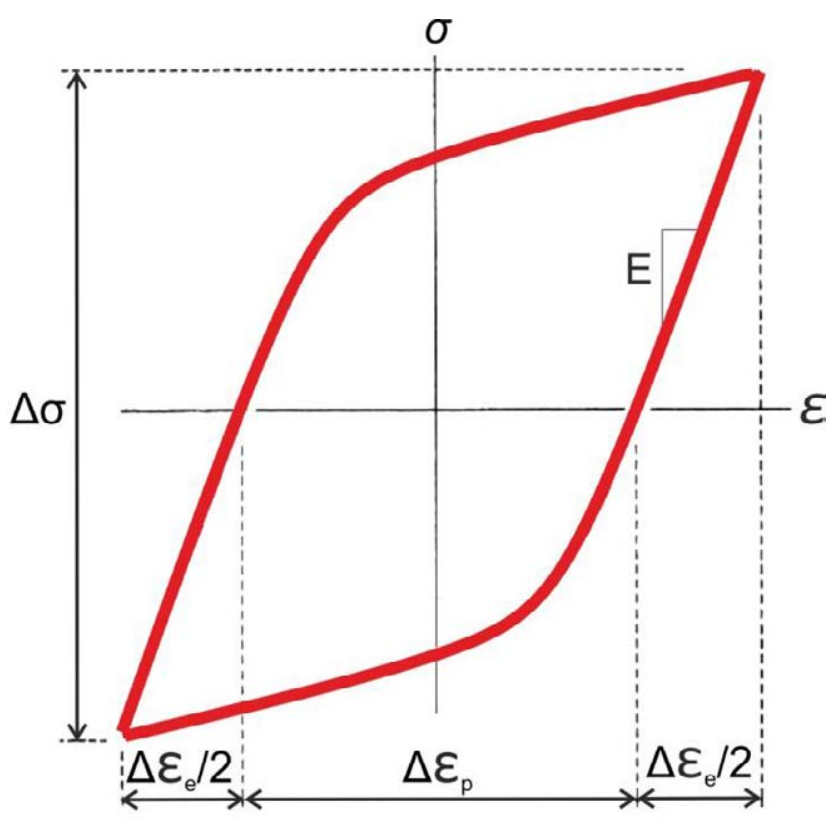

Figure 7 Strain hysteresis loop in fatigue load cycle.

Coffin and Manson first evaluated this effect, and observed that it is possible to represent the plastic deformation amplitude, $\Delta \varepsilon / 2$, versus the number of load reversals to failure in the logarithmic scale.

$$
\frac{\Delta \varepsilon_{p}}{2}=\varepsilon_{f}^{\prime}\left(2 N_{f}\right)^{c}
$$

Where $\Delta \varepsilon p$ is the total plastic strain on a complete cycle, $\varepsilon_{f}^{\prime}$ is the fatigue ductility coefficient, and $c$ is the fatigue ductility exponent.

On the other hand, based on Hooke's law, the elastic component of the load cycle hysteresis loop can be written as:

$$
\frac{\Delta \varepsilon_{e}}{2}=\frac{\Delta \sigma}{2 E}
$$

Where $E$ is the Young modulus of the material. Combining eq. (1.4) with Basquin's relation (1.2):

$$
\frac{\Delta \varepsilon_{e}}{2}=\frac{\sigma_{f}^{\prime}}{E}\left(2 N_{f}\right)^{b}
$$

Additionally, the total strain amplitude can be written as the sum of both the elastic and plastic component:

$$
\frac{\Delta \varepsilon_{t}}{2}=\frac{\Delta \varepsilon_{e}}{2}+\frac{\Delta \varepsilon_{p}}{2}
$$


Finally, substituting the expression proposed by Coffin Manson, and Basquin into eq. (1.6) it is obtained the total strain versus life relation.

$$
\frac{\Delta \varepsilon_{t}}{2}=\frac{\sigma_{f}^{\prime}}{E}\left(2 N_{f}\right)^{b}+\varepsilon_{f}^{\prime}\left(2 N_{f}\right)^{c}
$$

The first and second terms on the right hand side of eq. (1.7) represent the elastic and plastic contribution to fatigue failure respectively. This expression is also known as the CoffinManson relation, and forms the basis for the strain life approach. Figure 8 shows the graphical representation of the expression in logarithmic scale, together with the elastic and plastic components contribution.

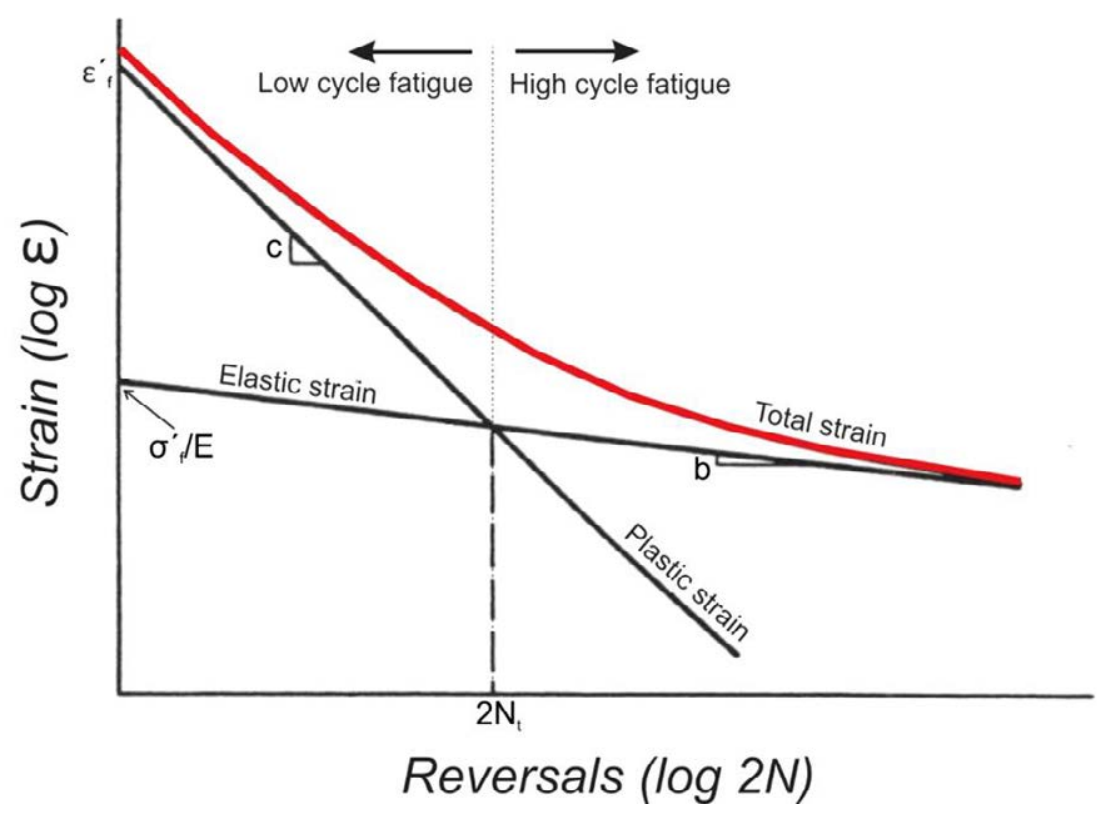

Figure 8 Strain based fatigue life.

It can be noticed in the figure the transition between the low and high fatigue life aforementioned. In the case of LCF, plastic strains are predominant, and therefore fatigue behavior will be controlled by the material ductility. On the contrary, for HCF the elastic strains will be more significant and the fatigue will be in turn dictated by the material fracture strength. The number of load inversions at which plastic and elastic contributions to fatigue are equal is known as the 'transition fatigue life' $\left(2 N_{t}\right)$, and can be deduced by equating eq. (1.3) and eq. (1.5):

$$
\varepsilon_{f}^{\prime}(2 N)^{c}=\frac{\sigma_{f}^{\prime}}{E}\left(2 N_{f}\right)^{b} \Rightarrow 2 N_{t}=\left(\frac{\varepsilon_{f}^{\prime} E}{\sigma_{f}^{\prime}}\right)^{\frac{1}{b-c}}
$$

Ultimately, the $\varepsilon-\mathrm{N}$ approach supposes a generalization of the $\sigma-\mathrm{N}$ approach in which the plastic strain contribution to fatigue is taken into consideration, making it a more complete method. Nevertheless, the complexity of the $\varepsilon-\mathrm{N}$ model is also increased since now the number 
of parameters to determine the strain-life curve are four $\left(\sigma_{f}^{\prime}, \varepsilon_{f}^{\prime}, b, c\right)$ against the two parameters needed in the $\sigma-\mathrm{N}$ model.

It can also be observed that for a sufficiently highly number of cycles, the plastic contribution to fatigue becomes negligible, making both $\varepsilon-\mathrm{N}$ and $\sigma-\mathrm{N}$ approaches to equate. Consequently, when a component design is limited to such number of cycles, the $\sigma-\mathrm{N}$ seems to be a more appropriate approach since it provides the same accuracy using a more simple model. On the other hand, when high strain cycles might be also present during the operation of the component, the $\varepsilon-\mathrm{N}$ approach becomes necessary to obtain reliable results.

\subsection{Linear Fracture Mechanics Based Approach}

The fatigue approaches presented in the last sections characterize the fatigue behavior of a component through the time or cycles up to failure. The Linear Fracture Mechanics approach differs a little from this idea: it focuses on studying an already cracked component to predict the crack propagation rate and thus the remaining life of the component. The investigations carried out in relation to this approach suppose an valuable information about the crack behavior and propagation phenomena.

This approach applies the elasticity theory, and is suitable to predict crack growth only during the stable propagation. It does not account for the material behavior during the initial crack nucleation phase, either during the last stage of fast propagation and failure. Therefore, it is not valid to estimate the total life of a non cracked component, where the initial phase of fatigue may take a significant number of cycles.

From the early sixties of the last century, many efforts were focused on relating the crack growth with the fatigue cycles. Cracked specimens were usually employed to perform characterization experiments, studying the influence on the crack growth rate of parameters like the material, crack shape or stress state. By way of example, Figure 9 shows the different crack growth behavior for three different stress ranges.

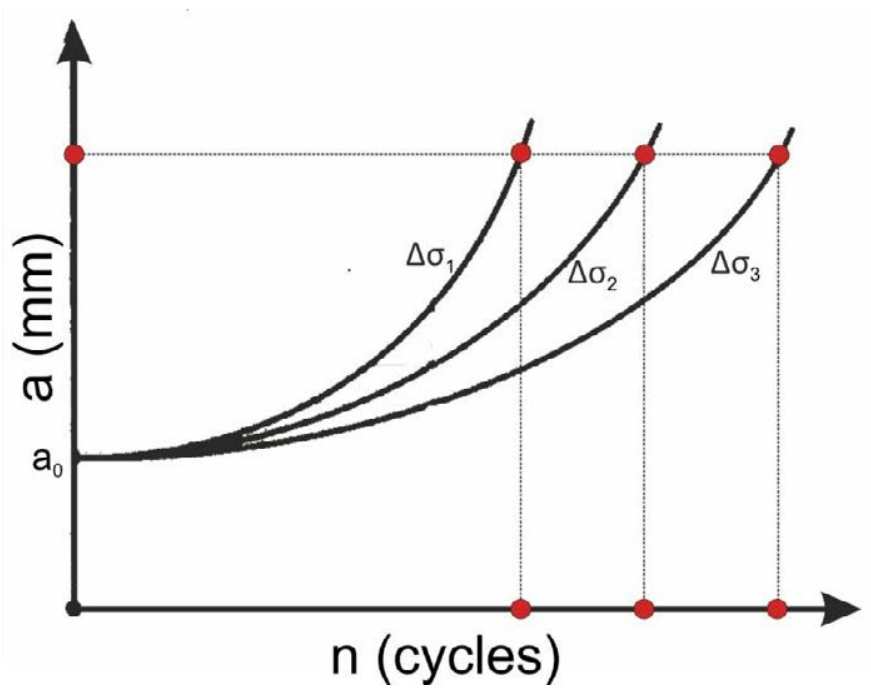

Figure 9 Crack growth for different stress ranges. 
Most of the investigations developed targeted to find an expression that predicts the crack growth rate $(d a / d n)$ in relation to the stress intensity factor increment $(\Delta K)$, which can be deduced from the maximum and minimum stresses during the load cycle as follows:

$$
\Delta K=\left(\sigma_{\max }-\sigma_{\min }\right) \alpha \sqrt{\pi a}
$$

The so-called geometric factor ' $\alpha$ ' is a coefficient that depends on the geometry of the components, the stress tensor in the vicinity of the crack tip and the size and position of the crack. An extensive database for this parameter was brought by D.P. Rooke and D.J. Cartwright (1974).

In this regard, Paris et al. (1961) were the first to propose an analytical relation between these two parameters:

$$
\frac{d a}{d n}=C_{p}(\Delta K)^{m_{p}}
$$

The expression (1.10) is known as "Paris equation", where the factors $C_{p}$ and $m_{p}$ are material dependent. It can be easily deduced that this expression corresponds to a straight line in the log-log scale. It shows good agreement to experimental results only in the stable crack growth phase (Figure 10).

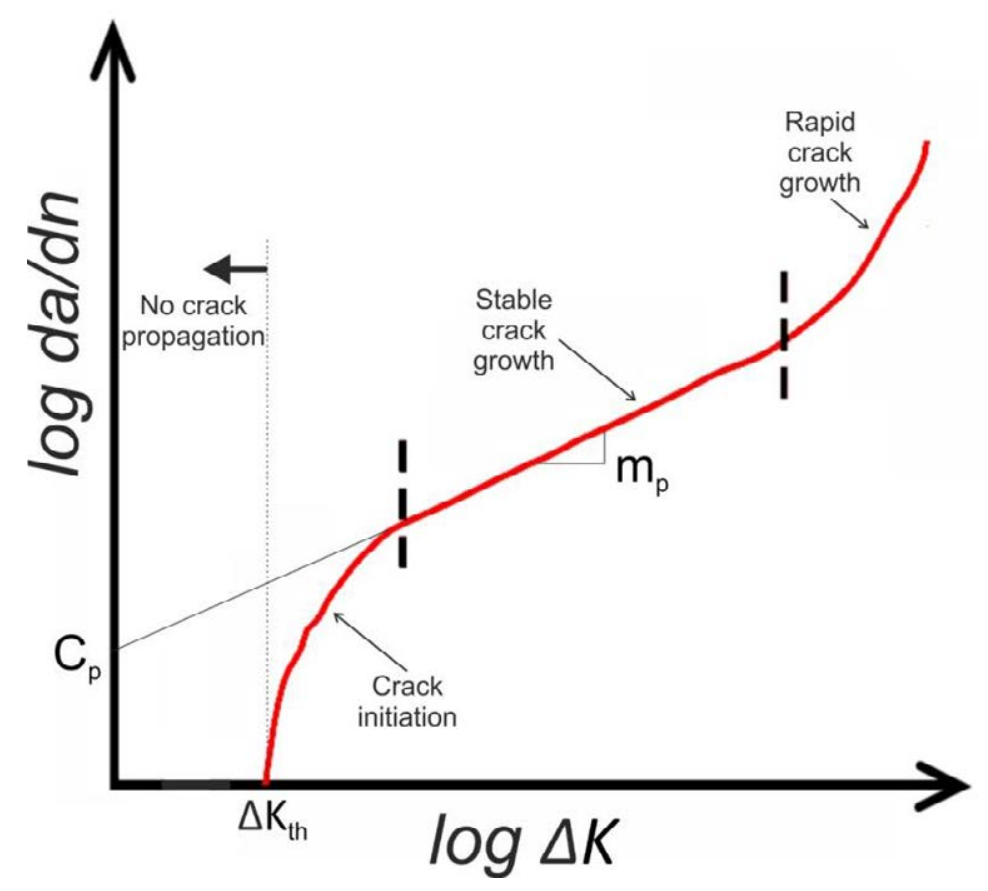

Figure 10 Crack growth rate versus stress intensity factor increment.

Finally, it should be pointed out that there is one threshold value for the stress intensity factor increment limit $\left(\Delta K_{t h}\right)$ below which the crack will not propagate. Clearly, this threshold value has direct relation with the fatigue limit $\left(\sigma_{e}\right)$. 


\subsubsection{Fatigue resistance modifying factors}

In general, the characteristics of the components and their working conditions differ significantly for those of the specimens for fatigue tests. Test specimens have a very well controlled geometry and a very fine surface finish and work in a very controlled environment, while actual mechanical components usually show rougher surfaces and might withstand corrosive or high temperature environments. These differences usually decrease the fatigue resistance of the component, and thus they must be considered in order to make and appropriate fatigue design.

There are two main approaches used to find the actual fatigue resistance of a component. One, more costly and accurate, is to manufacture the actual component and test it under similar conditions to those present during operation. The alternative is to apply a series of "modifying factors", empirically obtained, to adapt the fatigue resistance of the test specimens to the structural and functioning characteristics of the actual component. To this purpose, most common practice is to use the relation proposed by Marin (1962) to decrease the fatigue resistance of the specimen by the product of the different modifying factors:

$$
\sigma_{e(\text { part })}=\sigma_{e(\text { specimen })} K_{l} K_{f} K_{m} K_{t} K_{s}
$$

In the present thesis, for the sake of accuracy, the first alternative was followed, and actual welded junctions of bus structures where manufactured and tested under loads similar to those during normal operation. Nevertheless, it is considered relevant to present the principal fatigue modifying factors and its influence in fatigue life, since some of them are notably present in welded LPTV structures.

\subsection{Load type (K!)}

Reference fatigue specimens are tested by means of rotating bending loads, but actual operation of a component might include other type of load cycles like axial, torsion, or a combination of all of them.

Juvinall and Marshek (2000) proved experimentally that the load type factor takes a value close to 0.9 if the load is pure axial, while it can decrease up to 0.7 when it is combined with flexural loads according to Norton (2006). Shigley and Mischke (1990) studied the effect of flexural loads with additional torsion, recommending to adopt a factor of 0.923 or 1 , depending on if the ultimate strength of the steel is higher or lower than $1500 \mathrm{MPa}$ respectively. Regarding pure torsion loads, it is well accepted the Von Mises criterion, which corresponds to a factor of 0.577 according to the equivalent shear stress.

\subsection{Surface finish $\left(K_{f} L\right.$}

The fatigue crack usually initiate at the surface of the parts, since the superficial irregularities act as micro stress concentrators. Consequently, the higher the surface roughness, the less cycles will need the crack to initiate, decreasing the total life of the component. Figure 11 shows different surface finish factors for different steel specimens obtained experimentally. 
It is clearly noticed that more polished surfaces produce higher $K_{f}$ factors while, on the contrary, rougher finishes can lower the fatigue limit by up to $80 \%$. It can be also observed that higher strength steels show more sensitivity to the surface finish.

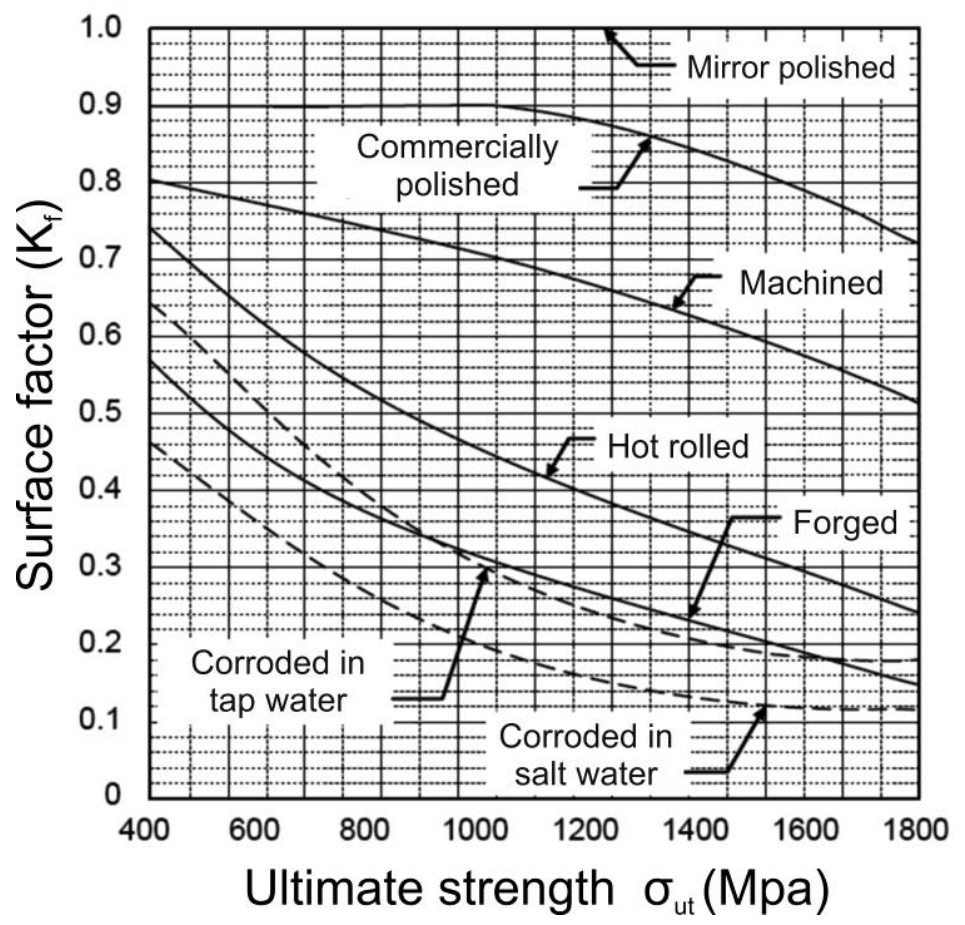

Figure 11 Surface finish factor (R.C. Juvinall and K.M. Marshek (2000)).

\subsection{Surface treatments $\left(K_{\underline{m}}\right)$}

Surface treatments encompasses both mechanical treatments and coating. In general the produce a positive effect on fatigue resistance, although the level of influence vary significantly depending on the treatment itself, the part geometry, and the operation characteristics.

Faupel and Fisher (1981) proposed the following analytical equation (1.12), where $Y$ adopts the values of Table 1 and Table 2 for shot peening and surface rolling mechanical treatments respectively. It can be observed that fatigue life improvement in already well finishes surfaces is less significant than in rougher surfaces.

$$
K_{m}=1+Y
$$

Table 1: Shot peening influence on fatigue resistance for different surface finishes

\begin{tabular}{|c|c|c|c|c|}
\hline & Polished & Machined & Rolled & Forged \\
\hline Y & $0.04-0.22$ & 0.25 & $0.25-0.5$ & $1-2$ \\
\hline
\end{tabular}

Table 2: Surface rolling influence on fatigue resistance for different materials

\begin{tabular}{|c|c|c|c|c|}
\hline & Steel shaft & Cast iron & Aluminum & Magnesium \\
\hline Y & $0.2-0.8$ & $0.2-1.9$ & $0.2-0.3$ & 0.5 \\
\hline
\end{tabular}


Among surface coating treatments, it is very usual to apply carburizing processes on the component, which produces a residual compressive stress with the subsequent positive effect on fatigue resistance. On the opposite, other coating treatments like nickel or chrome plated have a negative effect on fatigue resistance. Finally, galvanized parts do not show significant changes on fatigue life.

\subsection{Temperature $\left(K_{t}\right)$}

Mechanical properties of steels are closely linked to the temperature. At low temperatures they tend to increase the strength and become more brittle, while at temperatures over $500^{\circ} \mathrm{C}$ the fatigue resistance lowers notably, and the fatigue limits tends to disappear.

An approximate approach to quantify the influence of temperature on fatigue resistance, is to consider its variation equivalent to the variation of tensile strength with temperature:

$$
K_{t}=\frac{\sigma_{u t(T)}}{\sigma_{u t\left(20^{\circ} C\right)}}
$$

\subsection{Component size $\left(K_{s}\right)$}

Fatigue resistance shows a decreasing tendency when the part size increases due to three main reasons:

- Given any manufacturing technique, any bigger component will show more surface imperfections, micro cracks or inclusions than a smaller one.

- The volume of the part subjected to critical loads increases in a bigger part, and thus the probability of finding a defect to initiate the crack also increases.

- Manufacturing processes become more complicated in bigger parts due to the increasing difficulty for manipulations and inspecting the part.

Different analytical formulations have been proposed to relate the part diameter with the size factor, although none of them have thoroughly validated. By way of guidance it is possible to adopt the values presented in eq. (1.14). It should be noticed that the maximum part size is limited to $d=250 \mathrm{~mm}$, since the available data for bigger parts is very limited.

$$
\begin{array}{lll}
\text { Small } \quad(1 \leq d \leq 10) \mathrm{mm} & \rightarrow & K_{s}=1 \\
\text { Medium }(10 \leq d \leq 50) \mathrm{mm} & \rightarrow & K_{s}=0.85 \\
\text { Big } \quad(50 \leq d \leq 250) \mathrm{mm} \rightarrow & K_{s}=0.75
\end{array}
$$

For situations where components do not have circular cross section, an equivalent diameter $\left(\mathrm{d}_{\mathrm{eq}}\right)$ can be calculated for a rectangular section with cross dimensions $\mathrm{h}$ and $t$ undergoing bending from the following expression:

$$
d_{e q}^{2}=0.65 h t
$$




\subsubsection{Effect of mean stress}

Most of the fatigue tests obtained in laboratories from fatigue specimens, employ fully reversed loads i.e. zero mean stress on the load cycle. Nevertheless, in most of the occasions, mean stress values will not be null during actual operation of a component. It is therefore vitally important to determine the influence of nonzero mean stress load cycles.

A fatigue stress cycle is usually defined by the parameters shown in Figure 12 . Where $\sigma_{\max }$ and $\sigma_{\min }$ address to the maximum and minimum peak stress values over the cycle, while $\sigma_{m}$ and $\sigma_{a}$ correspond to the mean and alternate stresses. The later can be in turn determined from the peak stress values following equations (1.16) and (1.17) respectively.

$$
\begin{gathered}
\sigma_{m}=\frac{\sigma_{\max }+\sigma_{\min }}{2} \\
\sigma_{a}=\frac{\sigma_{\max }-\sigma_{\min }}{2}
\end{gathered}
$$

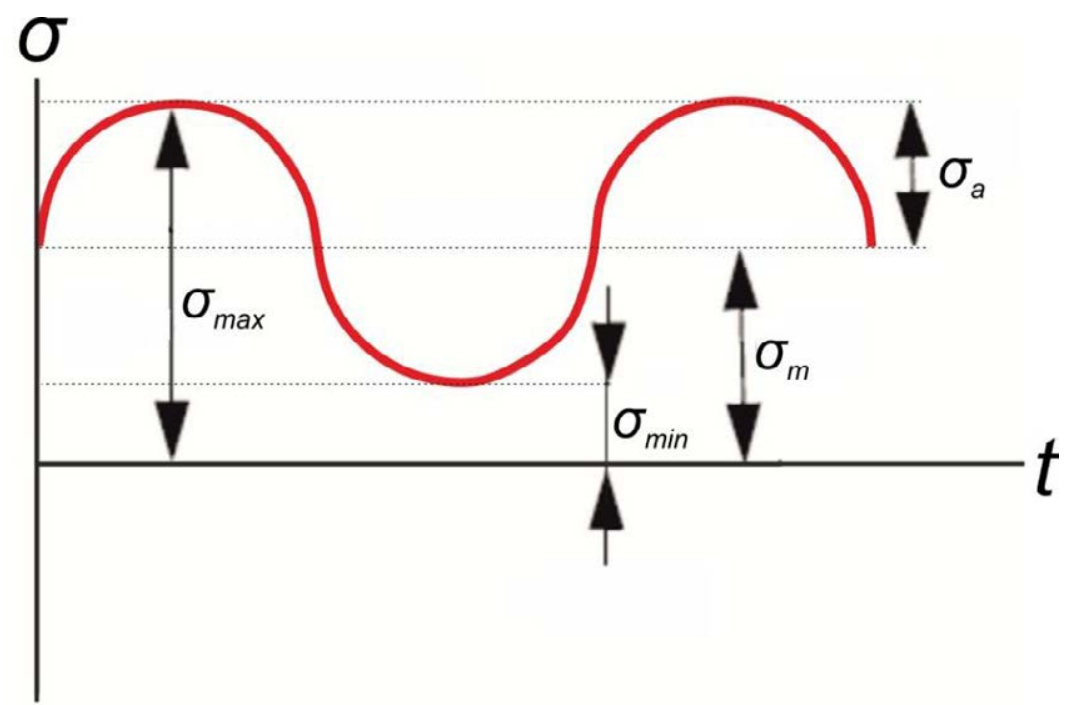

Figure 12 Fatigue cycle characteristic parameters.

In addition to this parameters, two ratios are also frequently used to represent fatigue cycle data:

$$
\begin{aligned}
& R=\frac{\sigma_{\text {min }}}{\sigma_{\text {max }}} \\
& A=\frac{\sigma_{a}}{\sigma_{m}}
\end{aligned}
$$

Where $R$ is so called the "Stress ratio", and $A$ the "Amplitude ratio". With this definition, the fully reversed bending fatigue tests described previously would correspond to a stress ratio $\mathrm{R}=-1$, and to an amplitude ratio $\mathrm{A}=\infty$. 
The following sections summarize the most important models employed to account for the effect of mean stresses on fatigue resistance for both $\sigma-\mathrm{N}$ and $\varepsilon-\mathrm{N}$ approaches.

\subsection{The effect of mean stress on the $\sigma-N$ approach}

As the mean stress increases, and the alternate stress keeps unchanged, the maximum stress will also increase, and therefore a fatigue life is expected to lessen. To quantify this effect, B.P. Haigh (1915) proposed to employ a diagram $\left(\sigma_{m}, \sigma_{a}\right)$, over which the fatigue limit for different combinations of mean and alternate stresses could be plotted. On these basis, different models have been proposed among which the most well known are the ones brought by Gerber (1874), Goodmann (1899) and Soderberg (1930), schematized in Figure 13.

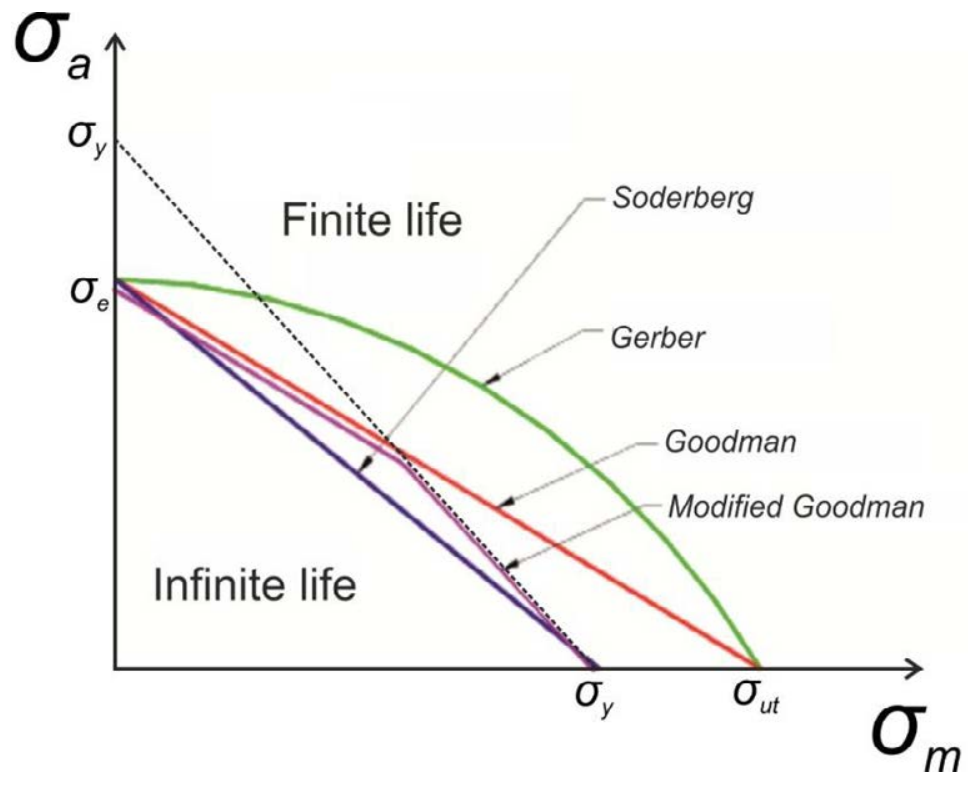

Figure 13 Mean stress effect models.

The parabolic criterion proposed by Gerber is the one that best matches the experimental observations, but also the least conservative, situating under its curve some failure points. Although most of these points can be attributed to test setup deviations like eccentricity on the loads or vibrations. According to this model, any point that meets eq. (1.20) will present infinite life.

$$
\frac{\sigma_{a}}{\sigma_{e}}+\left(\frac{\sigma_{m}}{\sigma_{u t}}\right)^{2} \leq 1
$$

On the other hand, Goodman proposed shortly after the line that today carries his name:

$$
\frac{\sigma_{a}}{\sigma_{e}}+\frac{\sigma_{m}}{\sigma_{u t}} \leq 1
$$

Experience has shown that actual test data tend to fall between the Goodman and Gerber curves. Nevertheless, these approaches do not consider failure in points that exceed the elastic limit $\left(\sigma_{y}\right)$. Although these stresses are usually not accepted in mechanical design of components 
and systems, Soderberg proposed a criterion based on the following line expression to overcome the dilemma:

$$
\frac{\sigma_{a}}{\sigma_{e}}+\frac{\sigma_{m}}{\sigma_{y}} \leq 1
$$

The Soderberg criterion eliminates thus the plastification issue, but at the cost of a considerably conservative approach. For this reason, a less conservative approach was developed to compound the higher accuracy of Goodman's line with the yield limit criterion. This combined solution is known as "modified Goodman" and writes as:

$$
\begin{aligned}
& \frac{\sigma_{a}}{\sigma_{e}}+\frac{\sigma_{m}}{\sigma_{u t}} \leq 1 \\
& \frac{\sigma_{a}}{\sigma_{y}}+\frac{\sigma_{m}}{\sigma_{y}} \leq 1
\end{aligned}
$$

It can be noticed that all criteria have been presented considering only positive mean stresses i.e. traction stress. In general terms, load cases with compressive mean stresses have a positive effect on fatigue life and thus the fatigue breakage in these situations is less common. Consequently, the efforts pointed to model the effect of mean stress on compressive states have been moderate. A frequently employed approach is to use symmetrical lines with the criteria presented before, which supposes the very conservative consideration of equivalent effect on fatigue of traction and compression loads. Another, less conservative approach proposed consists of considering no effect of compressive mean stresses on fatigue, but does considering yield failure, that is, an horizontal line in the negative $\left(\sigma_{m}, \sigma_{a}\right)$ up to the compressive yield line.

\subsection{The effect of mean stress on the $\varepsilon-N$ approach}

Experimental observations show that the effect of mean stresses is notably more significant at high cycle fatigue i.e. when elastic strains are predominant. The first model proposed to account for the mean stress effect in the strain-life approach was brought by Morrow (1968). He suggested taking into account this effect by modifying the elastic part of the Coffin-Manson relation by the mean stress $\left(\sigma_{m}\right)$ :

$$
\frac{\Delta \varepsilon_{t}}{2}=\frac{\left(\sigma_{f}^{\prime}-\sigma_{m}\right)}{E}\left(2 N_{f}\right)^{b}+\varepsilon_{f}^{\prime}\left(2 N_{f}\right)^{c}
$$

Or if preferred:

$$
\frac{\Delta \varepsilon_{t}}{2}=\left(\frac{\sigma_{f}^{\prime}}{E}-\varepsilon_{m}\right)\left(2 N_{f}\right)^{b}+\varepsilon_{f}^{\prime}\left(2 N_{f}\right)^{c}
$$


Graphical representation of the Morrow's approach is shown in Figure 14. Where it can be noticed the more significant influence of mean stresses on HCF. It should be pointed also that according to the model, a tensile stress would reduce the fatigue strength coefficient, whereas a compressive stress would increase it, and thus the fatigue resistance. This model have been extensively used for steels with special success in long life regime, where plastic amplitude is of little significance.

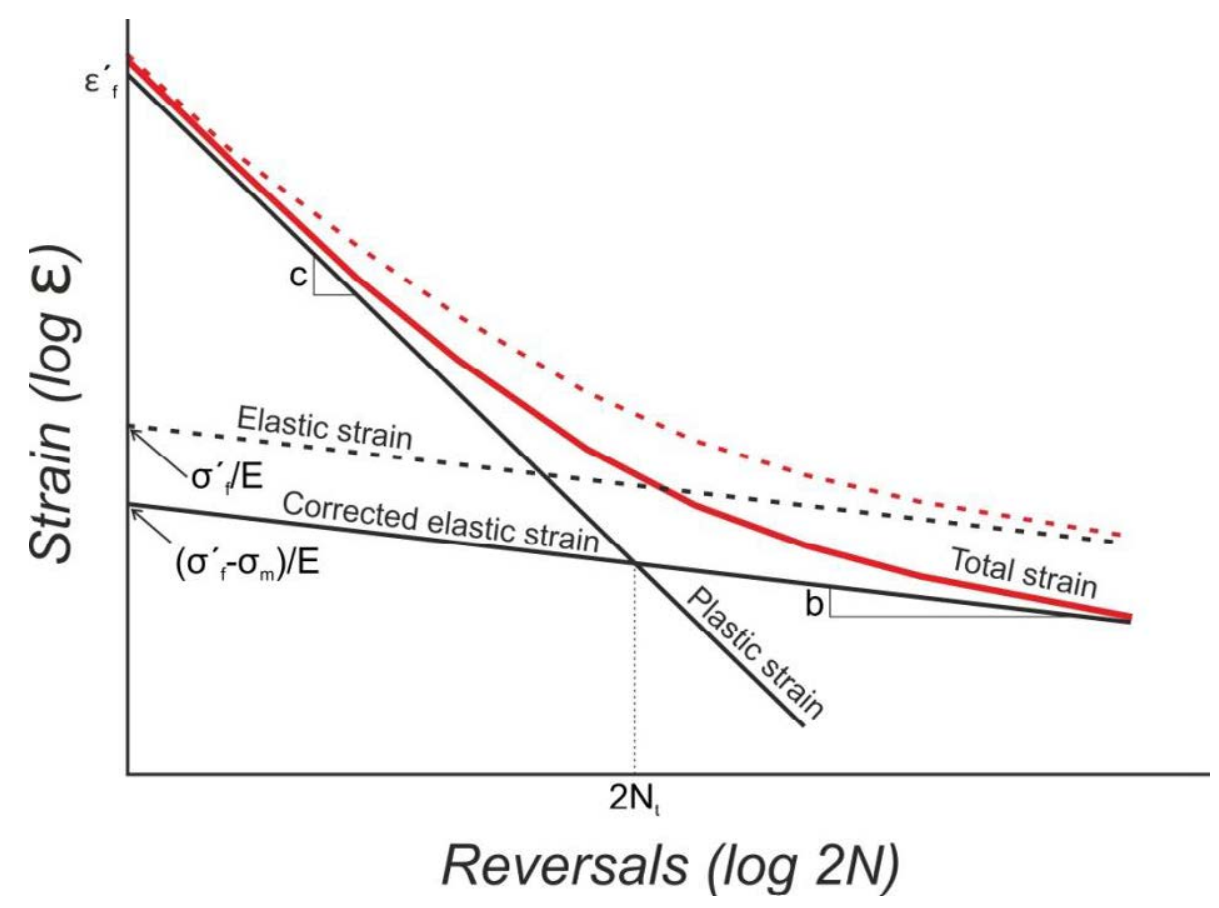

Figure 14 Morrow's mean stress correction.

An alternative model was proposed little after by K.N. Smith, et al. (1970). This approach, known as the SWT model, states that fatigue damage can be determined by the product of the strain amplitude $\left(\Delta \varepsilon_{t} / 2\right)$ and the maximum stress of the cycle $\left(\sigma_{\max }\right)$. Also, the model assumes that the product $\sigma_{\max } \cdot \Delta \varepsilon_{t} / 2$ remains constant for a specific number of cycles to failure $(N)$.

For fully reversed loads, the maximum stress coincides with the stress amplitude, so the Basquin equation can be written as:

$$
\sigma_{\max }=\sigma_{\mathrm{a}}=\sigma_{f}^{\prime}\left(2 N_{f}\right)^{b}
$$

Multiplying (1.26) by the strain life relation proposed by Coffin-Manson we obtain:

$$
\sigma_{\max } \frac{\Delta \varepsilon_{t}}{2}=\frac{\left(\sigma_{f}^{\prime}\right)^{2}}{E}\left(2 N_{f}\right)^{2 b}+\sigma_{f}^{\prime} \varepsilon_{f}^{\prime}\left(2 N_{f}\right)^{b+c}
$$

The SWT model is considered specially suitable for high strength steels and aluminum alloys. Nevertheless, it should be pointed that it predicts no fatigue damage when the maximum stress becomes zero or negative, which is not strictly true. 


\subsubsection{Cumulative damage approaches}

The approaches presented until now deal with constant amplitude loading. On the contrary, most operating service load histories present a variable amplitude and can be notably complex, where component will withstand a different $(k)$ amount of fatigue cycles $\left(n_{i}\right)$ at each different stress amplitude $\left(\sigma_{m}, \sigma_{a}\right)$ throughout the lifespan:

$$
n_{T}=\sum_{i=1}^{k} n_{i}
$$

Cumulative damage theories attempt to assess whether the component will present fatigue failure after the overall fatigue cycles supported $\left(n_{T}\right)$. They use damage fraction concept, defined as the life used up $\left(D_{i}\right)$ by a determined block of cycles $\left(n_{i}\right)$, and assume that failure will occur if the summation of all damage fractions is greater or equal to 1 :

$$
\sum_{i=1}^{k} D_{i} \geq 1
$$

The first damage rule was proposed by Palmgren in 1924 and further developed by Miner in 1945. This linear damage approach is today know as the "Palmgren-Minner rule" or sometimes just as the "Miner's rule". It states that the damage fraction at any supported stress level $\left(\sigma_{i}\right)$ is given by the relation between the number of cycles $\left(n_{i}\right)$ and the fatigue life at this stress level $\left(N_{i}\right)$ :

$$
D_{i}=\frac{n_{i}}{N_{i}}
$$

Consequently, the failure criterion could be written as:

$$
\sum_{i=1}^{k} \frac{n_{i}}{N_{i}} \geq 1
$$

The Miner's rule makes the following assumptions: first, that the sequence of application of the stress blocks does not affect in fatigue life, and second, that the damage accumulation is independent on the stress level.

Very good agreement to experimental observations has been observed when random or pseudo-random load histories are applied (J.A. Bannantine et al. 1990), which corresponds to the case study of the present thesis of the accelerations measured on LPTV vehicles as will be shown later.

On the other hand, Miner's rule tends to be non-conservative when higher stress cycles are first applied followed by blocks of lower stresses, and excessively conservative in the opposite case. Different nonlinear cumulative damage approaches have been proposed to 
improve the accuracy of Miner's rule in these situations, but its detailed description is out of the scope of the thesis.

\subsubsection{Multiaxial Fatigue Analysis Approach}

The previous chapters have introduced fatigue concepts based on uniaxial cyclic loading. However, in many situations, engineering components are subjected to more complex stress and strain states. Fatigue under these conditions is termed "multiaxial fatigue", and is a crucial consideration for reliable analysis and optimization of components and systems.

Multiaxial fatigue has been studied for the last hundred years, with an important work increase from the early 1970s. Despite the efforts, this area is still a topic of concentrated research nowadays, and no general consensus has been reached on the "best" theory, although some approaches have shown good agreement to experimental observations for specific applications and are very well accepted in the industry.

Within multiaxial fatigue, a distinction should be made between situations where the principal stress or strain directions remain constant (proportional loads) or not (nonproportional loads) during the load cycle, since different theories must be applied from one case to another. The difference between proportional and non-proportional loading is shown graphically for a biaxial strain state in the following Figure 15:

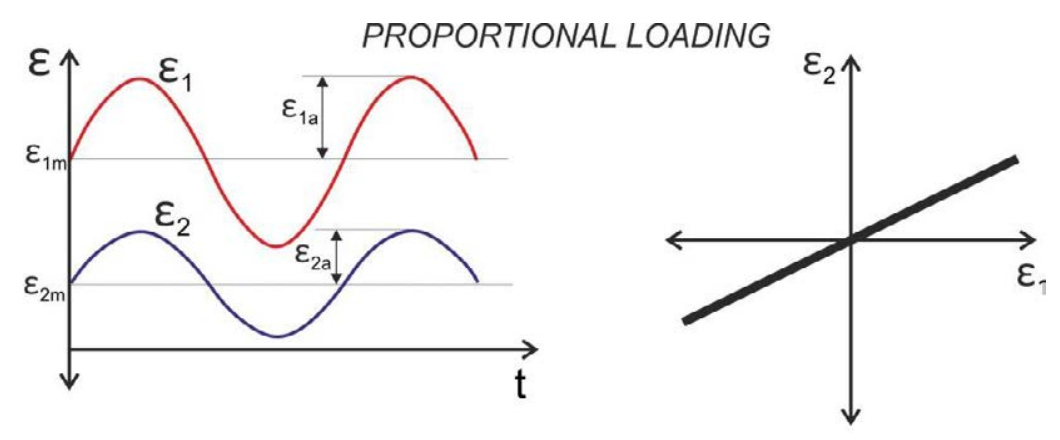

NON-PROPORTIONAL LOADING
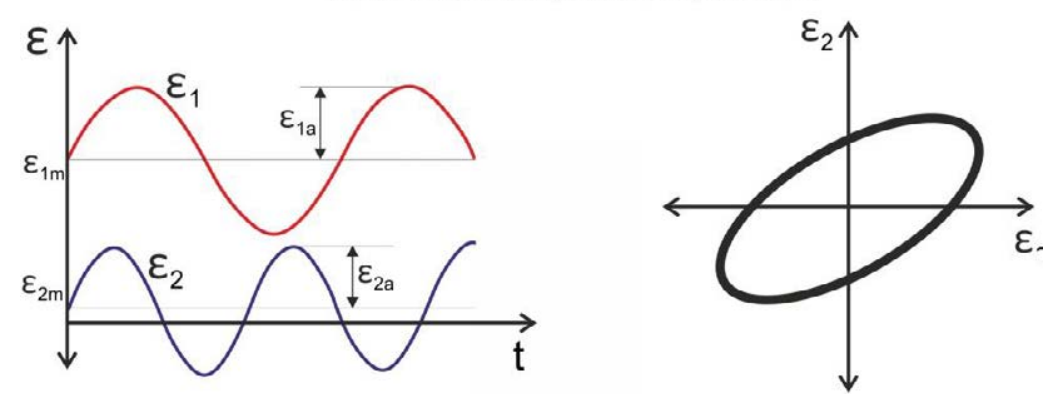

Figure 15 Proportional and non-proportional fatigue loads.

As it is shown, the relation of the principal strains remains constant during the load cycle in the case of proportional loading:

$$
\frac{\varepsilon_{1}}{\varepsilon_{2}}=c t e
$$


As will be shown later, the operating loads of LPTV structures produce predominantly bending forces that yield proportional multiaxial strain states at the strain concentration zone. Therefore, the multiaxial fatigue approach introduced in the current section will focus in this scenario.

In general terms, most multiaxial fatigue approaches are based on the determination of an equivalent stress or strain in order to substitute it by the stress or strain amplitude in Basquin or Coffin Manson equations respectively. Experimental observations have shown that employing the monotonic VonMises criterion to calculate this equivalent stress or strain present a good agreement to the actual behavior of specimens tested under proportional loading, especially in the HCF range. According to this approach, a material point (' $P$ ') subjected to fatigue loads will present failure due to distortion energy, following the Von Mises ellipsis for equal fatigue life under biaxial loading (Figure 16). This good agreement might be explained by the fact that fatigue cracks initiate due to shear stresses and strains, which is closely linked to the distortion energy criterion used to calculate the equivalent Von Mises stresses and strains.

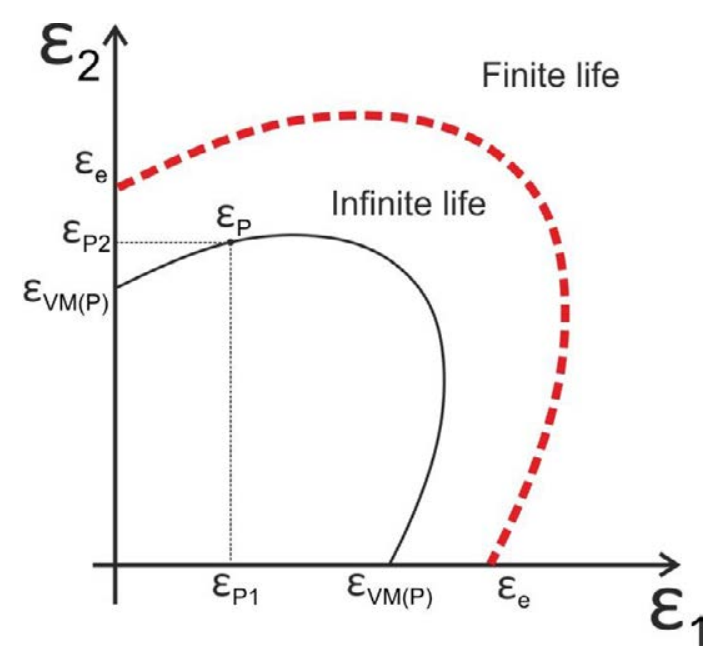

Figure 16 Equivalent uniaxial strains according to Von Mises criterion.

According to the figure, the equivalent Von Mises strain amplitude should verify the following inequality to ensure infinite fatigue life in a biaxial loading:

$$
\frac{\Delta \varepsilon_{t}}{2}=\varepsilon_{V M}=\frac{1}{\sqrt{2}(1+v)}\left[\varepsilon_{1}{ }^{2}+\varepsilon_{2}{ }^{2}-\varepsilon_{1} \varepsilon_{2}\right]^{0.5} \leq \varepsilon_{e}
$$

In order to differentiate tension and compression strain states, the most employed approach is to evaluate the sign of the hydrostatic stress. When this stress invariant is positive, traction load state is assumed and Von Mises will remain positive, while negative hydrostatic stresses will count as compressive states, and the Von Mises value will change sign to negative. This criterion is shown graphically in the following Figure 17. 


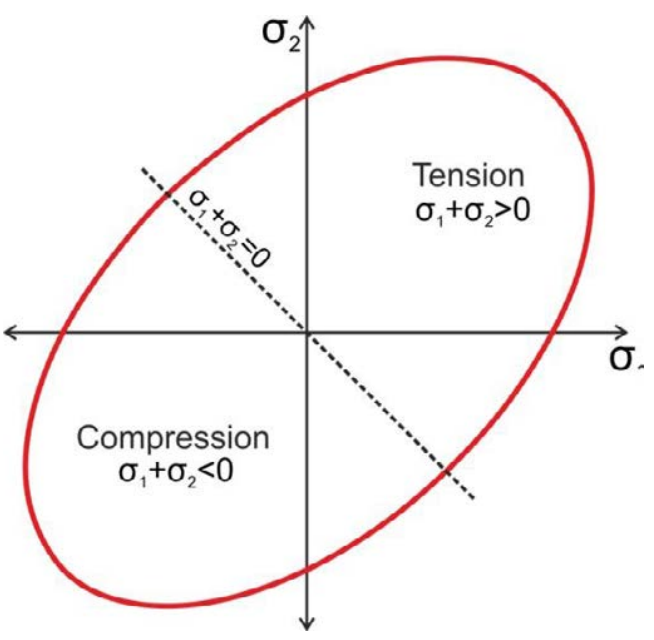

Figure 17 Von Mises sign criterion.

The process of application of this methodology to the strain-life approach with the mean stress correction proposed by Morrow (1.24) is schematized in Figure 18. As shown, the equivalent mean strain of the load cycle needs to be determined to apply the nonzero mean strain correction, and the equivalent strain amplitude as the strain input to obtain the number of reversals to failure.

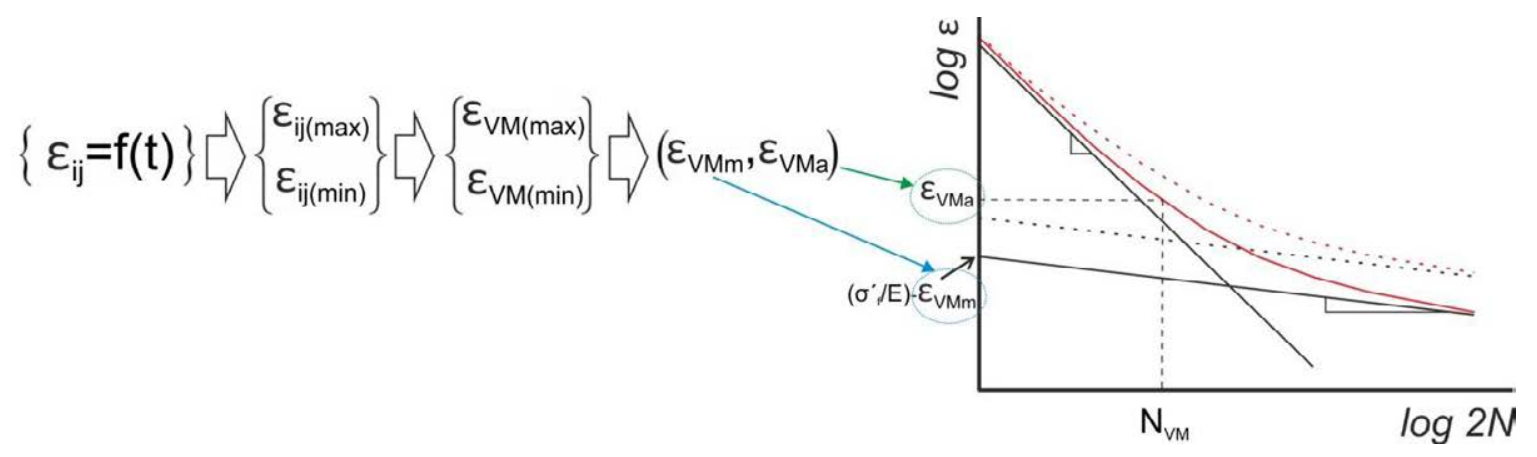

Figure 18 Application of the equivalent Von Mises strain criterion to multiaxial fatigue with proportional loading.

Any load cycle will in turn produce a strain cycle, with its respective maximum and minimum strain states. The Von Mises equivalent strain values for these maximum and minimum states can be subsequently calculated by means of eq. (1.34) and (1.35) respectively. It is worth to mention that the path followed by the strain cycle was found to have little influence on fatigue of steels in comparison to the peak values for frequencies under $200 \mathrm{~Hz}$ (I. Nonaka, et al. (2013)). Therefore, this methodology does not take into account the shape of the load cycle and performs the fatigue the evaluation only considering peak values.

$$
\begin{aligned}
& \varepsilon_{V M(\max )}=\frac{1}{\sqrt{2}(1+v)}\left[\left(\varepsilon_{1(\max )}-\varepsilon_{2(\max )}\right)^{2}+\left(\varepsilon_{2(\max )}-\varepsilon_{3(\max )}\right)^{2}+\left(\varepsilon_{3(\max )}-\varepsilon_{1(\max )}\right)^{2}\right]^{0.5} \\
& \varepsilon_{V M(\min )}=\frac{1}{\sqrt{2}(1+v)}\left[\left(\varepsilon_{1(\min )}-\varepsilon_{2(\min )}\right)^{2}+\left(\varepsilon_{2(\min )}-\varepsilon_{3(\min )}\right)^{2}+\left(\varepsilon_{3(\min )}-\varepsilon_{1(\min )}\right)^{2}\right]^{0.5}
\end{aligned}
$$


From these values, mean and alternate equivalent strains can be directly obtained by means of eq. (1.16) and (1.17). Finally, the mean component $\left(\varepsilon_{m}\right)$ will have a reducing effect on the Coffin-Manson curve and the alternate component $\left(\varepsilon_{a}\right)$ will be used as the input strain amplitude to obtain the number of reversals to failure $\left(2 \mathrm{~N}_{\mathrm{f}}\right)$, as written in the following equation:

$$
\varepsilon_{V M(a)}=\left(\frac{\sigma_{f}^{\prime}}{E}-\varepsilon_{V M(m)}\right)\left(2 N_{f}\right)^{b}+\varepsilon_{f}^{\prime}\left(2 N_{f}\right)^{c}
$$

\subsubsection{CYCLE COUNTING METHOD: RAINFLOW}

Fatigue load cycles acting on components are not always as clear to estimate or measure as in the examples presented in the previous sections. Very often, load histories are not known in advance, and acquisition equipments are necessary to measure them. The recorded loads usually have complex variation (similar to Figure 19 (a)) and the determination of load cycles from them is not direct.

Fatigue evaluation in these conditions requires the development of a process that enables obtaining the fatigue load cycles and thus the posterior assessment of the component in terms of fatigue damage.

Different cycle counting methods have been proposed in the last fifty years. They all aim to extract load sequences or blocks of constant mean and amplitude, and follow the assumptions that neither load rate or load cycle shape has significant effect on fatigue behavior of the component.

One of the most successfully used methods is the so-called 'Rainflow' cycle counting method, originally developed by Matsuishi \& Endo (1968), from which a number of variations have been proposed. Among these variants, the most well known are the three-point cycle counting techniques (included in the SAE Standard (1997), or ASTM E1049 Standard (1985)), and the four point cycle counts methods (Amzallag et al., 1994). Both lead to identical results (C.H. Mclnnes \& P.A. Meehan, 2008) and differences reside only in the cycle computation process. By way of example, the three point counting method will be described in this section.

Prior beginning the cycle counting process itself, some re-arrangement of the load history signal must be performed. First, all data that does not belong to load peaks or valleys should be discarded (Figure 19(b)). A load peak is defined as a point whose first derivate changes sign from positive to negative, equivalently, a valley is defined as a point whose derivate changes sign from negative to positive. Second, the load series must be reordered to begin and end with the overall maximum as shown in Figure 19 (c). 


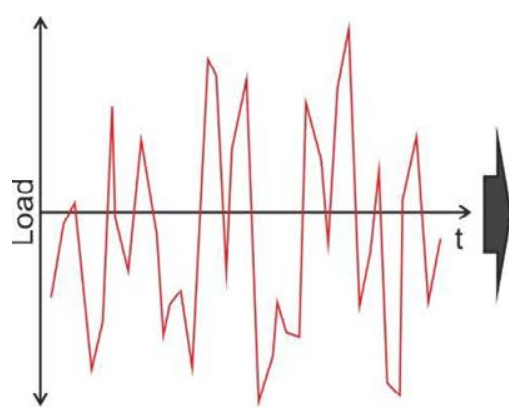

(a)

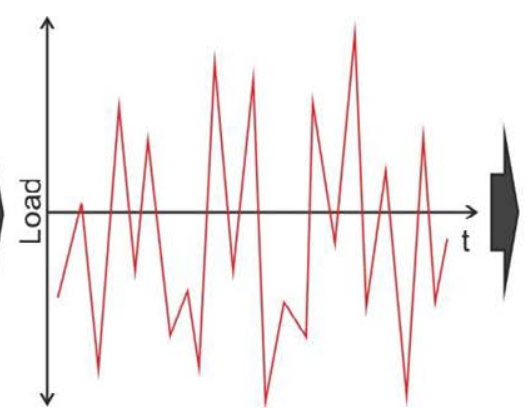

(b)

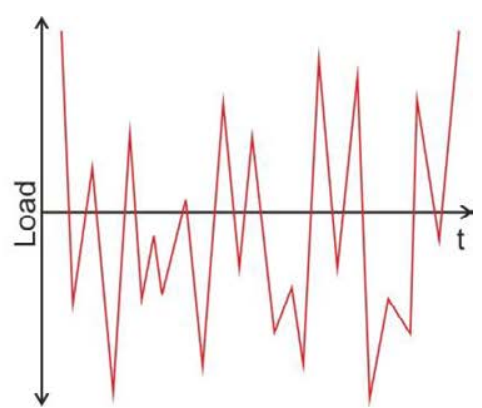

(c)

Figure 19 Time series rearrangement. (a) Original load history. (b) Peak and valleys of the load history. (c) Rearrangement to start with the overall maximum.

Once performed the initial arrangement, the load history would be ready to run the cycle counting algorithm. The three-point rainflow method evaluates 3 consecutive points obtaining two ranges. The first range is the result of the absolute difference between the first and second points evaluated. Analogously, the second range is the absolute difference between the second and third points. The condition to consider a cycle between the evaluated points is that the value of the first range is lower than the second one. If a cycle is localized, first and second evaluated points are removed from the load history and a new iteration starts. For a better understanding, the process is shown bellow step by step for the simple load history of Figure 20.

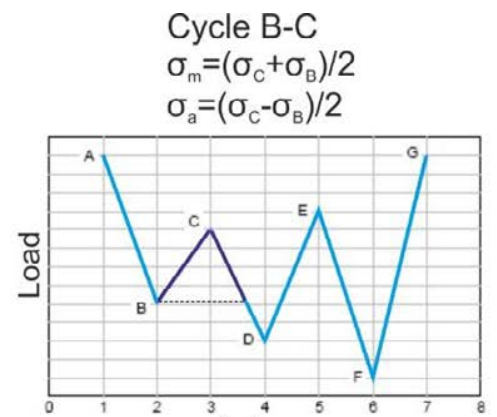

(a)

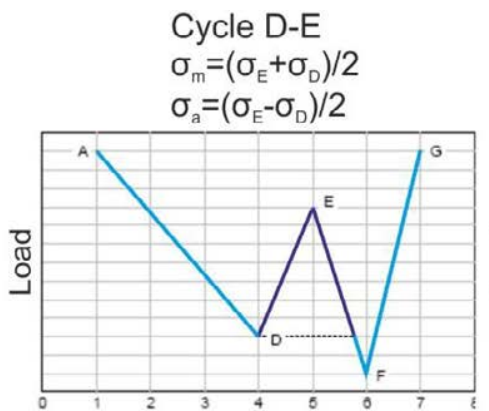

(b)

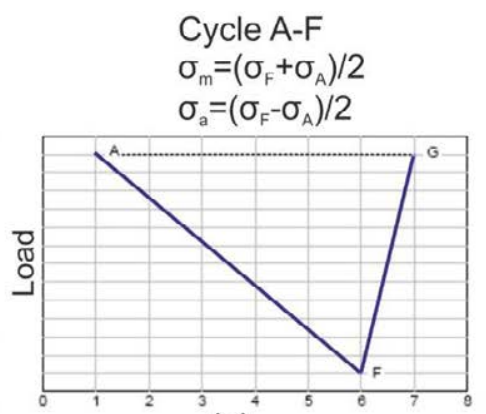

(c)

Figure 20 Three point Rainflow application example.

The steps carried out by the algorithm to extract the load cycles are the following:

1. Points A-B-C: $\left|\sigma_{B^{-}} \sigma_{A}\right|>\left|\sigma_{C^{-}} \sigma_{B}\right|$, no cycle, move to points $B, C$ and $D$.

2. Points B-C-D: $\left|\sigma_{C^{-}} \sigma_{B}\right|<\left|\sigma_{D^{-}} \sigma_{C}\right|$, cycle B-C localized, $\sigma_{m}$ y $\sigma_{a}$ are determined as shown in Figure 20 (a). $B$ and $C$ points removed from load history. Move to points $A, D$ and $E$.

3. Points A-D-E: $\left|\sigma_{D^{-}} \sigma_{A}\right|>\left|\sigma_{E^{-}} \sigma_{D}\right|$, no cycle, move to points $D, E$ and $F$.

4. Points D-E-F: $\left|\sigma_{E^{-}} \sigma_{D}\right|<\left|\sigma_{F^{-}} \sigma_{E}\right|$, cycle D-E localized, $\sigma_{m} y \sigma_{a}$ are determined as shown in Figure 20 (b). D and $E$ points removed from load history. Move to points $A, F$ and $G$.

5. Points A-F-G: $\left|\sigma_{F^{-}} \sigma_{A}\right|<\left|\sigma_{G^{-}} \sigma_{F}\right|$, cycle A-F localized, $\sigma_{m} y \sigma_{a}$ are determined as shown in Figure 20 (c). Only point $\mathrm{G}$ remaining, less than the three needed for a new iteration so routine is completed.

It should be noted that the load history presented in this example was prepared in advance so that no rearranged was needed prior starting the cycle counting algorithm, since the history already started and finished at the overall maximum, and all its points were peaks or 
valleys. It is characteristic from this cycle count method to obtain the highest amplitude cycle in the last iteration.

\subsection{FINITE ELEMENT METHOD FOR FATIGUE LIFE ASSESMENTS}

Very frequently, the complex geometry and loads of the component under evaluation makes almost impossible to estimate accurately the critical strains that will suffer during operation by means of traditional materials strength theories, leading to higher safety factors and thus to not optimized solutions. The notable development of Finite Element Methods (FEM) in the last years shows itself as a powerful alternative to overcome these drawbacks. This technique, joined with the increasing computing capabilities, enables more precise calculations and evaluations of the designed products. Additionally, since FEM is a virtual based simulation technique, it is possible to perform optimizing processes before manufacturing and testing the actual products, with the consequent costs and time savings.

Fatigue evaluations using FEM can be understood as a post processing operation, in which the outcomes of the simulation are employed to perform any of the fatigue assessments described in the previous sections. Therefore, the accuracy of these outcomes becomes crucial for a reliable fatigue life assessment. In turn, the accuracy of the results of a FEM calculation will depend on the precision of the inputs, the model quality and the solver algorithms.

A basic schema of a component fatigue design using FEM is shown in Figure 21. First, the inputs of the model need to be defined. In global terms, they are three: the geometry (discretized in a finite amount of small elements), loads and materials. Further detail on these three inputs will be given in the next subsections. Once these inputs have been defined, the virtual model can be solved by means of the specific solver algorithm needed. Finally, the fatigue analysis can be performed for the obtained results. If the result meets the design requirements the process finishes and the project can move to the next milestone, if not, redesign would be needed. To help in the new iteration, this type of analysis spots the most critical areas, giving valuable information about where the design improvements should be focused.

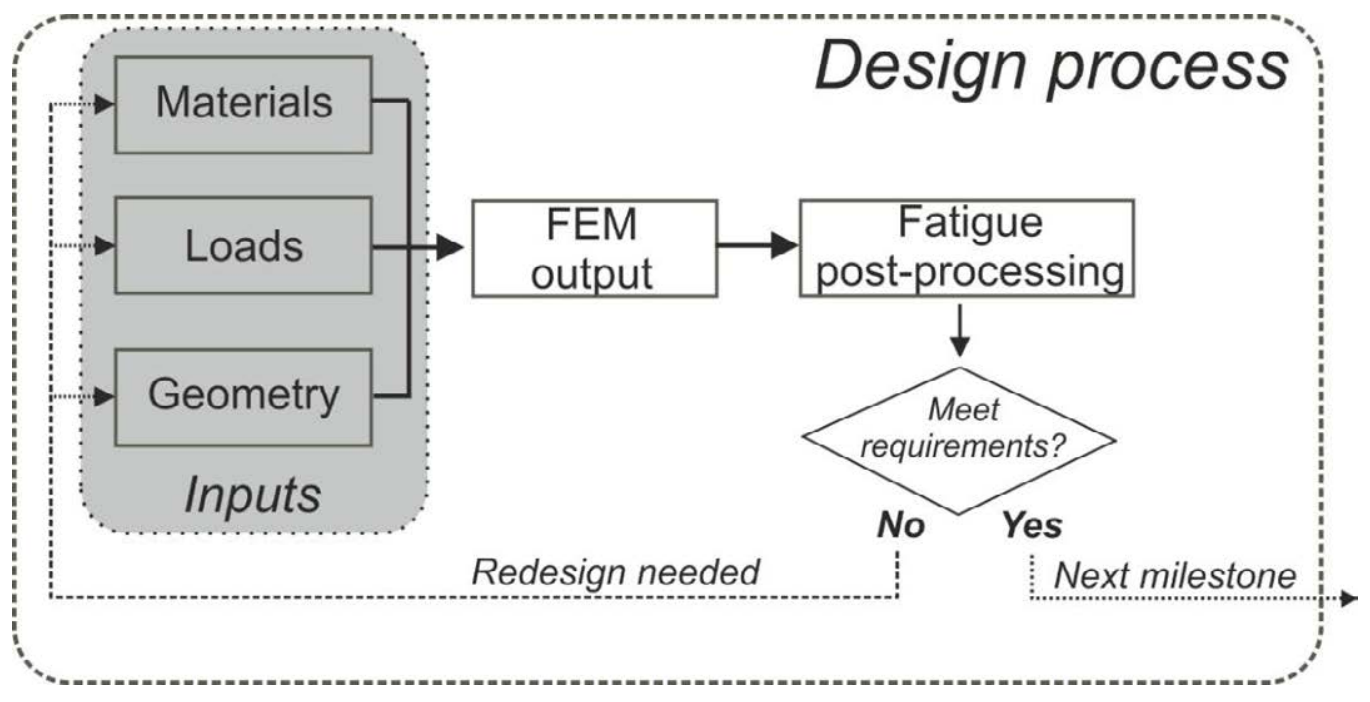

Figure 21 FEM based fatigue design process. 


\subsubsection{FINITE ELEMENT METHOD BASICS}

The Finite Element Method (FEM) can be defined as a numerical technique to approximate physical problems governed by differential equations. Its basic idea is so divide a continuous physical domain into a discretized finite number of more simple and interconnected domains, called elements, so that its governing equations can be written down and later solved simultaneously.

The elements are assumed to be connected by nodes, which are located at the edges and vertices of them. Therefore, the method can be also understood as the technique to approximate the domain response by solving the governing equations at a discrete number of points or nodes. In a general structural problem, the governing equation is written as:

$$
[M][\ddot{u}]+[C][\dot{u}]+[K][u]=[F]
$$

Where $M, C$ and $K$ are the mass, damping and stiffness matrices respectively, $F$ is the applied external forces and $u$ the state-vector response and unknown of the equation.

A significant amount of commercial FEM software products exist nowadays, covering a vast range of market needs from simple linear static problems, to highly nonlinear dynamics ones such as explosion simulations. Despite of the wide variety of commercial codes and physical problems to solve, FEM usually follows the next three principal steps:

- Preprocessing: In this first phase, the geometry model is constructed, to later divide it in elements and nodes through a process called 'meshing'. Some of these nodes will be prescribed with the proper loads and boundary conditions (i.e. imposed displacements, velocities, etc). Also material properties must be given at these elements during this phase. The preparation of the model can be significantly time consuming and is of vital importance for obtaining reliable results.

- Analysis: the information of the previously prepared model is used to construct and solve the equilibrium equations (1.37). The total number of equations is the number of degrees of freedom of the model. In the case structural models constructed with three dimensional solid type elements, the total number of DOF is three times the number of nodes of the model. Depending on whether the mass, damping or stiffness matrices changes throughout the simulation or not, the model is considered nonlinear or linear respectively. Increasing the number of DOF (i.e. mesh refinement), or adding nonlinearities, increases the complexity and thus the computational cost of the analysis.

- Postprocessing: From the nodal displacements obtained during the problem analysis, the stresses, strains, or other derived data can be calculated. For a clear interpretation of the results, modern commercial software display them as colored contours over the model shape to represent the different levels of the output variable shown. 
In FEM structural analysis, depending on the geometrical characteristics of the model as well as the precision needed, the elements employed for the discretización of the geometry can be of different types. Although a profound study on the capabilities and applications of each element type is out of the scope of the present thesis (deeper descriptions can be found in specific books like in R.L. Zienkiewicz \& O.C Taylor (2000), J.N. Reddy (2005) or V. Adams \& A. Askenazi (1999)), a general description of its main characteristics is listed below:

- Volume or solid elements: Are the most general elements in FEM analysis, since they are valid to study any type of structure or component. There are three main types of solid elements (Figure 22): tetrahedral, prismatic and hexahedral. Each of these elements can be in turn classified by its formulation order as linear, if nodes exist only at the elements vertices, or quadratic, if additional nodes are places on the edges of the element. Each node on solid elements has 3 DOF, corresponding to the three spatial displacements; the rotation of the elements is deduced by the composition of its nodal displacements.

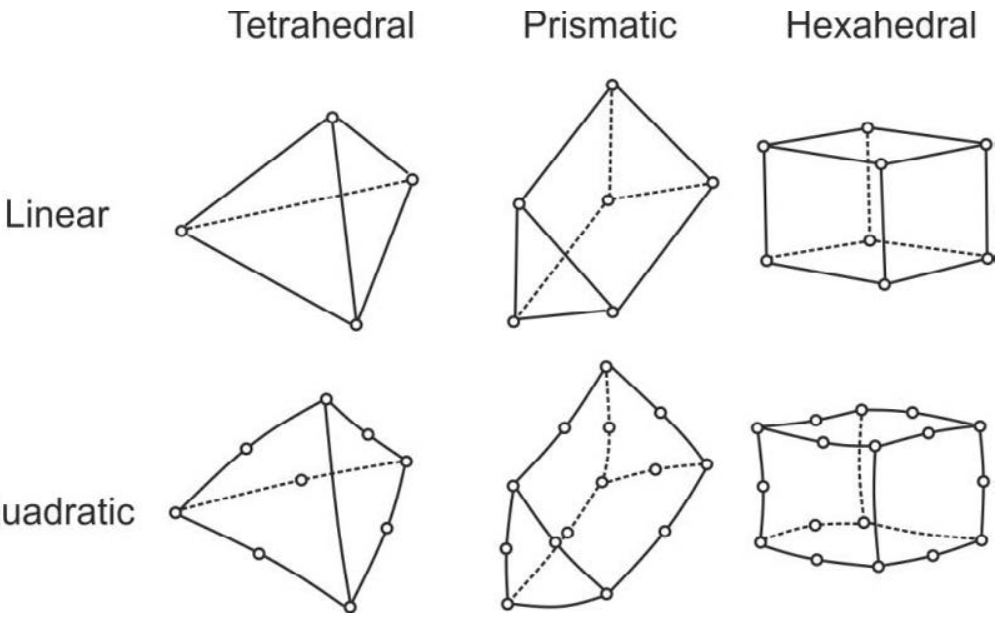

Figure 22 Volume or solid element types.

- Surface or shell elements: They are considered as a simplification of the general solid elements, they are suitable for model geometries where one of the dimensions is very small with respect to the other two. This simplification compels the user to input the missing geometrical information i.e. shell thickness. The most common types of shell elements are triangular or quadrilateral elements, and they can also have linear or quadratic formulations. Shell element nodes have $6 \mathrm{DOF}$, that corresponding to the three displacements and rotations on the space.

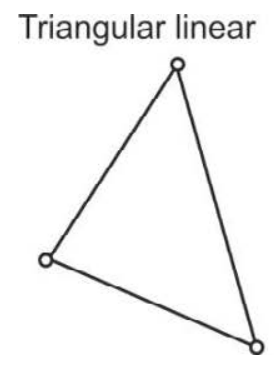

Triangular Quadratic

Quadrilateral lineal

Quadrilateral Quadratic
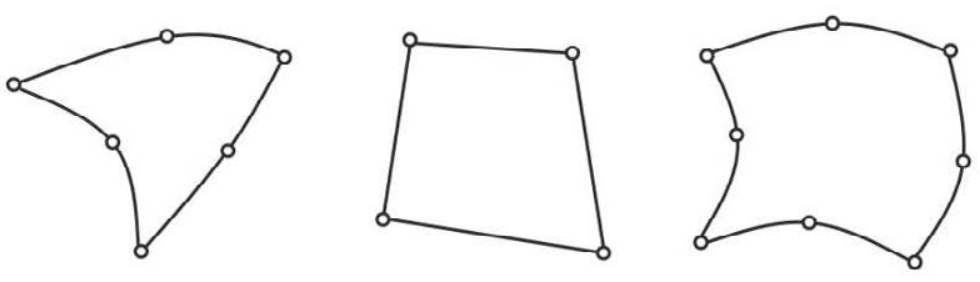

Figure 23 Shell element types. 
- Beam elements: these elements are in turn a simplification of shell elements, and are suitable for model geometries where two of the dimensions are very small with respect to the other one. Since these elements are one-dimensional, additional information about the other two must be input (i.e. cross sections in tubular structures). Most element formulations available in commercial software usually include an additional node or point to define the orientation of this cross section. Also linear or quadratic formulations can be found for beam type elements (Figure 24), and each node has 6 DOF as in the case of shell type elements.

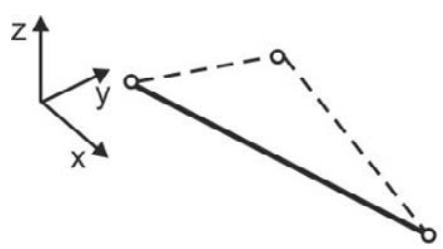

Beam Linear

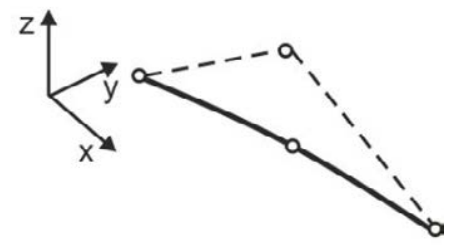

Quadratic Linear

Figure 24 Beam element types.

\subsubsection{LOAD INPUT}

By definition, any fatigue load is variable with respect to time, and therefore response of the structure or component will be dynamic. Nevertheless, for the sake of analysis simplicity, these cyclic loads are usually simplified to quasi-static ones during the model construction. This simplification entails eliminating any time dependent influence on the structure response i.e. the first two terms in eq. (1.37), obtaining the following simplified expression:

$$
[K][u]=[F]
$$

In fatigue analysis using FEM, it is common to simplify the continuous time varying load cycle by two quasi-static load cases corresponding to the maximum and minimum peak values (Figure 25). As stated before, these peak values are the ones of interest for fatigue analysis.

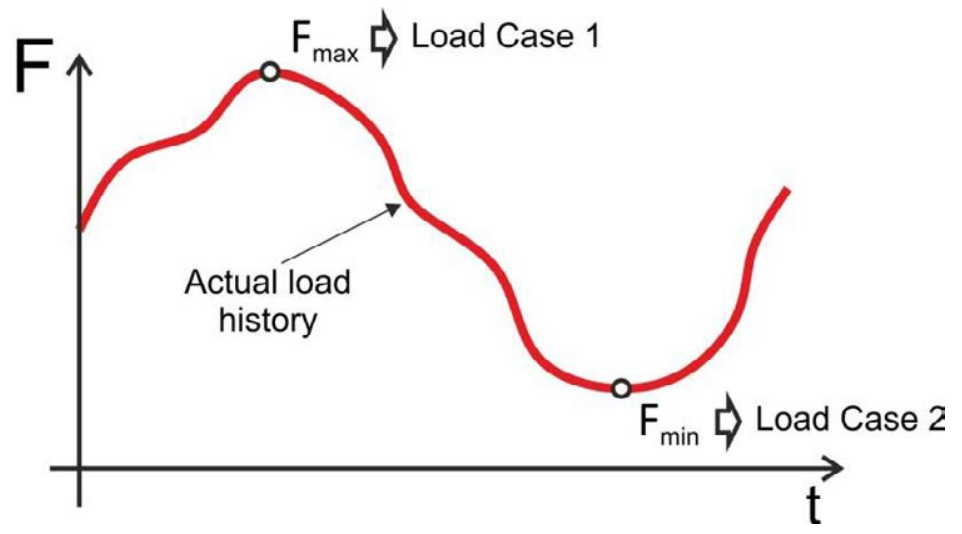

Figure 25 Quasi-static load simplification.

In any case, before adopting such simplifications, is important to evaluate the possible influence of dynamic effects on the results. The first term of eq. (1.37) refers to the inertial 
forces, and reads as mass times acceleration. It can be easily deduced that a heavier component would need less acceleration to produce significant inertial forces. On the other hand, heavier components are also likely to be more rigid and thus the deformations suffered under same loading would be less.

The second term of eq. (1.37) refers to the damping forces of the structure, and it is characterized by means of the damping coefficients matrix times the velocity (or frequency) of load application. As shown in Figure 26, the response of a structure loaded at frequencies close to its natural vibration mode can be amplified and in turn the stresses suffering by this structure will increase notably, especially in the case of low damping ratio $(\xi<<1)$. For damping coefficients close to 1 (critical damping) or higher (over-damped), the structure shows less sensible to the frequency of load application, so quasi-static simplification can be adopted for higher frequency solicitations.

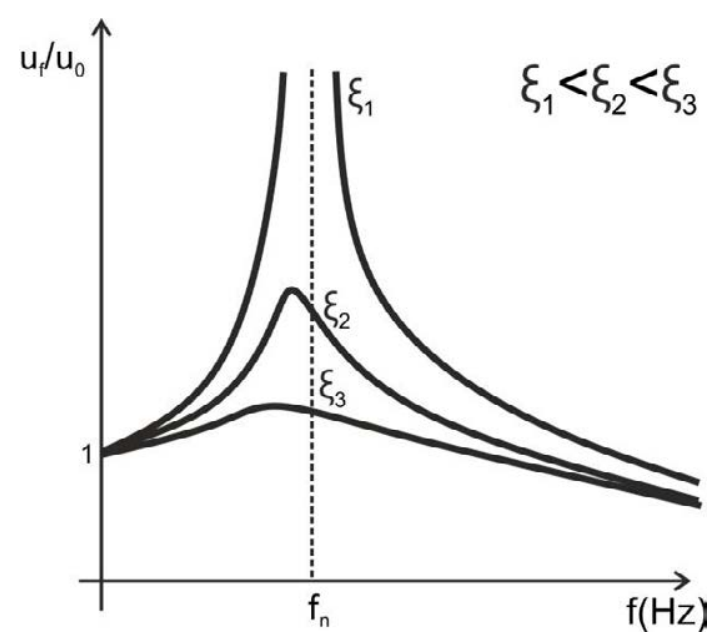

Figure 26 Damping influence on dynamic response.

In summary, dynamic effects on the response of a structure are always present in fatigue loads, and its magnitude is dependent on both structure and load characteristics. There is no general rule for whether accounting or not for these effects in fatigue analysis, but the decision should be consciously done depending on the aforementioned characteristics as well as the precision needed and the time available for the analysis.

\subsubsection{MATERIAL MODEL}

In FEM, the mathematical models used to describe the stress-strain relationship are called material models. The different parameters used to describe the material models are usually determined by data fitting using material test data.

Structural steels are isotropic non-linear plastic materials. A typical stress-strain relation under monotonic loading for a structural steel is shown in Figure 27 (a). Nonetheless, for calculation simplicity, the material models typically used in fatigue and structural analysis employ different levels of idealization:

- Linear elastic behavior(green line in Figure 27): is the most simplified material model. It is defined by Hooke's law and does not entail any material non-linearity. 
It therefore supposes the least computational cost of all material models. On the other hand, these models are only valid for stresses under the yield point of the material $\left(\sigma_{y}\right)$. It is widely used since this yield point is a very extended limit criteria in structural design.

- Bilinear elastic-plastic behavior (blue line in Figure 27): considers a non-linear material whose behavior is idealized by two lines, referring to the elastic and plastic behavior respectively. The transition between elastic to plastic areas supposes a change in the material stiffness and consequently in the stiffness matrix. This non-linearity entails additional iterations during the solution that complicate the process. Although these material models show higher accuracy that the linear one for stresses and strains beyond the yield point, it still shows significant deviations from reality at stress levels close to this point.

- Multi-linear elastic-plastic behavior (red line in Figure 27): this material model is equivalent to the previous described bilinear model, but instead of using two lines to characterize the whole stress-strain range, a set of lines are used. The more lines are defined to approach the real flow curve of the material, the more accurate the structure behavior will be; but on the other hand, the calculation process will have to deal with more stiffness changes and thus the number of convergence iterations will increase, slowing down the calculation process.

- Constitutive material models (orange line in Figure 27): these type of material modes attempt to describe the material behavior by means of a continuum relations. In this field, the relations brought by P. Ludwik (1909), Ramberg-Osgood (1943), J.H. Hollomon (1949) or H. Swift (1952) are remarkable examples of still great use nowadays.

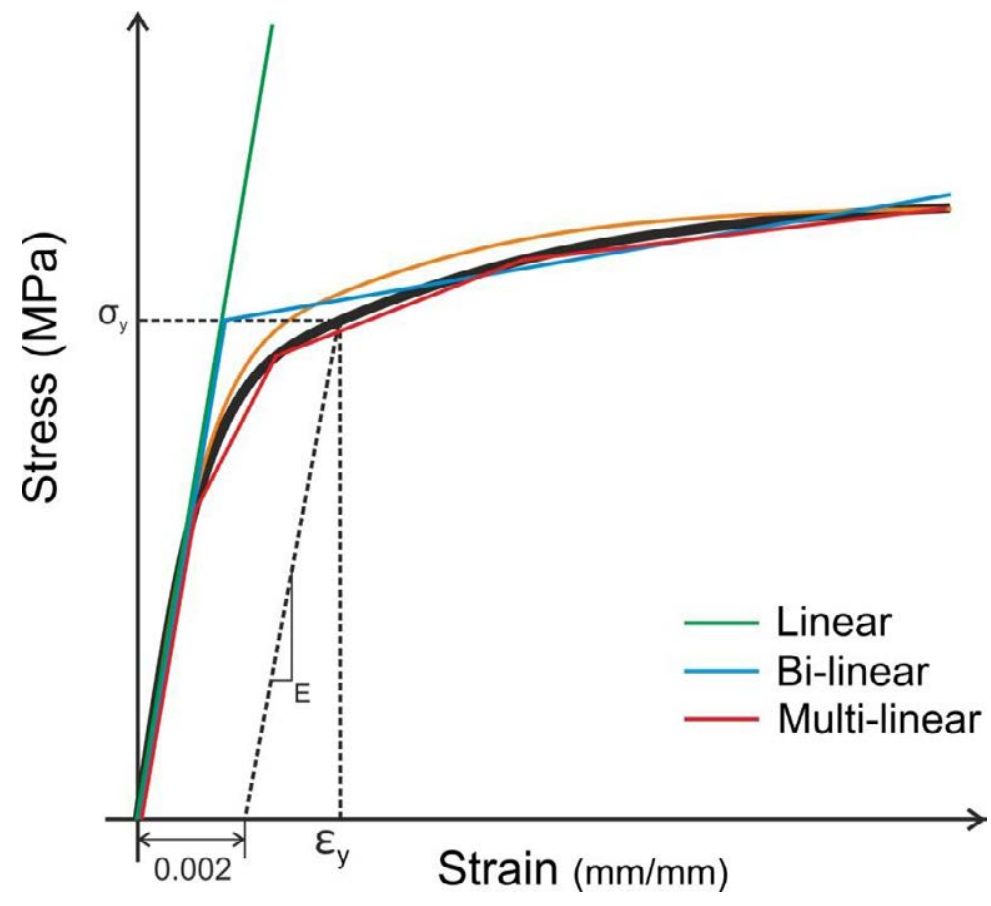

Figure 27 Types of material idealization. 
In some fatigue FEM analysis, apart from the monotonic stress-strain relation, there might be additional influences on the material behavior of high frequency and cyclic loading.

\subsubsection{CALCULATING LOCAL STRAINS IN WELDED JUNCTIONS}

As stated before, geometry must be discretized my means of the so-called 'meshing' process. In the case of a solid body, the geometry is divided in small hexahedral or tetrahedral elements as shown in Figure 22.

During the models solving, the displacements are determined at node locations $(u)$ which are later interpolated by means of the shape function to obtain the displacement fields $(d)$ :

$$
[N][u]=[d]
$$

Where $N$ is the so called matrix of shape functions. For first order elements the shape functions are linear, whereas for second order elements these functions are quadratic.

Finally, the displacement fields are derived at the integration points (usually gauss points) to obtain the strains and stresses on the structure.

It can be easily deduced from this reasoning, that the more elements form the model mesh, the more accurately it will describe its behavior. On the other hand, a small element size implies using a larger number of elements, and the computational resources and time needed to solve the model can increase significantly.

In the case of welded joints, the maximum local strains at the weld toe are highly dependent on the local geometrical characteristics, thus entailing the construction of very fine mesh at these regions if reliable results are desired. As we move away from these areas, the deformation becomes more homogeneous and thus larger elements can be used with no accuracy loss.

Among the different approaches of finite element modeling techniques of welded junctions, two main tendencies can be differentiated. On the one hand, there are techniques that do not consider (or consider in a simplified manner) the weld bead itself, and estimates the maximum stresses or strains by means of extrapolation formulae from nodal data at specific distances from the weld toe as schematized in Figure 28 (O. Doerk et al. (2003), W. Fricke (2001), ). This type of modeling offers a possibility of accounting for concentration effects from a relative simple models, although it presents some important drawbacks:

- Entails specific post-processing to obtain the maximum strains

- The extrapolation equations are based on experimental data, which is limited to some specific types of welding techniques and loading conditions.

- Cannot account for specific weld geometries such as corners in butt welds of rectangular beams, which, as will be shown later in the thesis, show the maximum strains of the junction. 


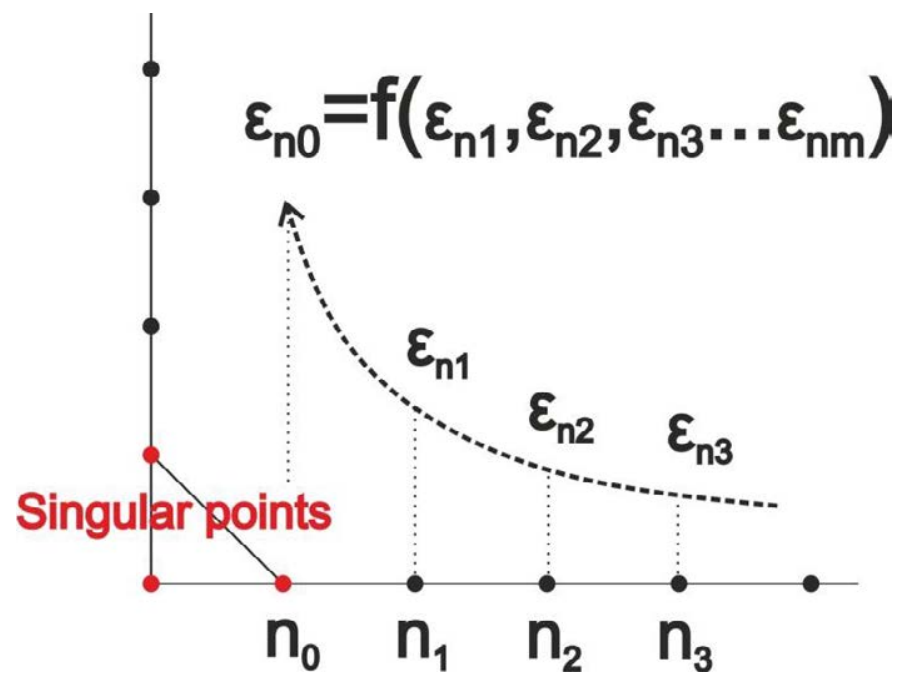

Figure 28 Strain extrapolation.

On the other hand, other modeling techniques aim to obtain the strain concentration behavior by including the geometric detail of the weld bead in the model (C.Y. Hou (2007), T. Mert (2009), A. Fouathia et al. (2014)). These type of modeling employ very fine meshes at the vicinity of the weld toe. In order to avoid working with excessively heavy models, submodeling techniques are commonly used in such detailed geometries (W. Fricke (2010)).

\subsubsection{REVIEW OF BUS BODY STRUCTURES MODELLING TECHNIQUES}

When the model gets more complex and the number and type of welded junctions becomes larger, as is the case of complete LPTV body structures, defining a FEM model at a very detailed level becomes an very laborious task (F. Badea et. al (2014)). Consequently, it is very common to resort on simplified modeling techniques based on beam type elements.

In this situation, most of the studies carried out on complete LPTV structure models focus on its global response. O. Ruiz et. al (2012), for example, presented an optimization process based on the output stresses in a beam type element model after proving the difficulties associated to a solid mesh for the whole structure. S. Manokruang and S. Butdee (2009) also used a beam type element model to implement an optimization process of a LPTV body based on the response comparison of the structure in terms of stiffness and stresses. A similar modeling procedure was proposed by D. Shinabuth et al. (2012) to analyze the structure of an electric bus body. In this work, stresses values were analyzed on the structure when subjected to different high severity operation maneuvers.

Although the utilization of this element type for LPTV modeling has a widespread acceptance, it shows some limitations, such as the impossibility of considering the geometrical characteristics of welded joints. These limitations produces erroneous strain and stress outcomes at the critical points of the welded junctions (i.e. local strain concentrations) and therefore outcomes from these type of models should be treated with special care.

With the improvement of the FEM software products and the increase of the computers capabilities, some models of complete bus body structures based on shell type elements arose. 
C.C. Liang and G.N. Le (2012), for example, presented a bus model based on 4-noded shell elements to perform a lightweight optimization concerning rollover safety. H. Hu et. al (2012) also relied on shell type elements to validate a finite element model of a transit bus by means of modal analysis.

Although shell element type models can reproduce the response of the structure more precisely than beam based models (F. Badea et. al (2014)), they also show clear limitations when predicting peaks stresses at welded joints due to the existence of singular points at the joints modeled this way. Consequently, both beam and shell element type models might fairly predict the general response of the structure, but are inaccurate in local maximum stress or strain outputs and cannot be taken as absolute rules for design criteria.

In any case, the level of detail that an accurate model of a welded junction demands makes the construction of a complete bus body an extremely costly task, both from the model preparation and from the computational points of view. In this scenario it is not surprising that the existing bibliography is found to approach separately the detailed local analysis of welded junctions from the global analysis of complete bus structures.

Considering that fatigue failure in LPTV structures is most likely to occur at the strain raises of the weld toe, these modeling techniques cannot be considered as a realistic option for fatigue assessments on such structures.

To overcome this limitation, this thesis develops a complete correlation process between beam and validated solid detailed models of typical welded junctions in LPTV structures.

\subsection{EXPERIMENTAL METHODS TO OBTAIN STRENGTH AND FATIGUE PROPERTIES ON WELDED JUNCTIONS}

This chapter aims to describe the different experimental techniques for the characterization of strength and fatigue properties of LPTV welded structures.

As it was mentioned before, $\mathrm{S}-\mathrm{N}$ fatigue life approach is the most simple method and is valid for most application within automotive industry since most designs are performed based on HLF or even infinite life criteria. Nevertheless, in notched components, i.e. welded junctions, local plastic deformations are likely to be present and therefore the strain-life approach becomes necessary to account for this nonlinearity.

Since the behavior of the material is no longer linear in the strain-life approach, some knowledge of the elastoplastic properties of the material becomes necessary to fully asses fatigue resistance. Test based characterization of these characteristics are detailed in the subsequent sections.

\subsubsection{MONOTONIC AND CYCLIC STRESS-STRAIN CURVES}

Monotonic stress-strain curve is generally obtained by means of uniaxial tensile test performed on tensile machines. This test consists of subjecting a geometrically controlled 
specimen to tensile loads that slowly increase up to breakage. The standards ASTM E606 and ASTM A739 provides a good description and recommendations for of the specimen preparation, test procedure and data analysis.

From the monotonic tensile test, different properties of the material that supply quantitative information about its capacity to resists loads (strength) and to strain (ductility) can be obtained. Figure 29 represents the most relevant data the can be extracted from this curves.

The engineering stress $\left(\sigma_{e n g}\right)$ is determined using the original cross-sectional area $\left(A_{0}\right)$ of the specimen, and reads as the relation between this area and the instantaneous tensile force applied:

$$
\sigma_{\text {eng }}=\frac{P}{A_{0}}
$$

Ultimate engineering stress $\left(\sigma_{u(e n g)}\right)$ can be therefore determined as:

$$
\sigma_{u(e n g)}=\frac{P_{\max }}{A_{0}}
$$

Analogously, the engineering strain $\left(\varepsilon_{\text {eng }}\right)$ is determined using the original length $\left(I_{0}\right)$ of the specimen, and reads as the relation between the instantaneous length and the original length:

$$
\varepsilon_{\text {eng }}=\frac{l-l_{0}}{l_{0}}=\frac{\Delta l}{l_{0}}=\lambda-1
$$

Where $\lambda$ is defined as the stretch gradient $\left(1 / I_{0}\right)$.

Ultimate engineering strain $\left(\varepsilon_{u(e n g)}\right)$ can be then obtained as:

$$
\varepsilon_{u(\text { eng })}=\frac{l_{\max }-l_{0}}{l_{0}}=\frac{\Delta l_{\max }}{l_{0}}
$$

Both stress and strain characteristics are of special interest to determine material properties regardless the dimensions of the component. These two properties can be related by means of the Hooke's law within the elastic range (i.e. stresses below $\sigma_{y}$ ):

$$
E=\frac{\sigma_{e n g}}{\varepsilon_{e n g}}
$$

Where $E$ is the Young or elastic module. This relationship is no longer valid beyond the yield point, which is commonly defined as the stress associated with a $0.2 \%$ plastic strain, as shown in Figure 29. 
To obtain the true stress $\left(\sigma_{\text {true }}\right)$, actual cross sectional area $(A)$ at each instant must be used instead of using the initial one.

$$
\sigma_{\text {true }}=\frac{P}{A}
$$

On the other hand, true or logarithmic strain $\left(\varepsilon_{\text {true }}\right)$ is defined as the instantaneous elongation per unit length of the specimen, thus accounting for the instant specimen length $(I)$ instead of the initial one:

$$
\varepsilon_{\text {true }}=\int_{l_{0}}^{l} \frac{d l}{l}=\ln \frac{l}{l_{0}}=\ln (\lambda)
$$

Assuming no volume change during tensile test, engineering and true stresses and strains can be related as follows:

$$
\begin{gathered}
\frac{A_{0}}{A}=\frac{l}{l_{0}}=\frac{\Delta l}{l_{0}}+\frac{l_{0}}{l_{0}}=1+\varepsilon_{\text {eng }} \\
\sigma_{\text {true }}=\frac{P}{A} \frac{A_{0}}{A_{0}}=\sigma_{\text {eng }}\left(1+\varepsilon_{\text {eng }}\right) \\
\varepsilon_{\text {true }}=\ln \frac{l}{l_{0}}=\ln \left(\frac{\Delta l}{l_{0}}+\frac{l_{0}}{l_{0}}\right)=\ln \left(\varepsilon_{\text {eng }}+1\right)
\end{gathered}
$$

It can be easily deduced that the differences between engineering and true stress-strain curves increase as the load and elongation increases. For practical applications where maximum deformations are close to the yield point or below, these differences are almost indistinguishable.

Both engineering and true strain measures are linear with respect to stretch of the material. Additionally, there are other proposed of strain measures widely used in engineering. One of them is the Lagrangian or Green strain, which is measured as follows:

$$
\varepsilon_{G L}=\frac{1}{2}\left(\frac{l^{2}-l_{0}^{2}}{l_{0}^{2}}\right)=\frac{1}{2}\left(\lambda^{2}-1\right)
$$

Also, Eulerian or Almansi strain measurer is defined as: 


$$
\varepsilon_{E A}=\frac{1}{2}\left(\frac{l^{2}-l_{0}^{2}}{l^{2}}\right)=\frac{1}{2}\left(1-\frac{1}{\lambda^{2}}\right)
$$

It is noted that the different strain measures can be easily related by means of the so called stretch gradient $\lambda$.

In ductile steels, the monotonic true stress-strain curve is commonly formulated by means of equations that separate elastic $\left(\varepsilon_{e}\right)$ and plastic $\left(\varepsilon_{p}\right)$ components of the total strain. The most widely used is written as eq. (1.52) and was first proposed by W. Ramberg \& W.R. Osgood in 1943. For simplicity, true stress $\left(\sigma_{\text {true }}\right)$ and true strain $\left(\varepsilon_{\text {true }}\right)$ will be referenced as stress $(\sigma)$ strain $(\varepsilon)$ from now on.

$$
\varepsilon=\varepsilon_{e}+\varepsilon_{p}=\frac{\sigma}{E}+\left(\frac{\sigma}{K}\right)^{1 / n}
$$

Where $n$ is the strain-hardening exponent and $K$ is the strength coefficient and they describe the stress-plastic strain relationship. This relation is valid in the range of deformations below the start of necking (i.e. approximately from $\sigma_{u(e n g)}$ )

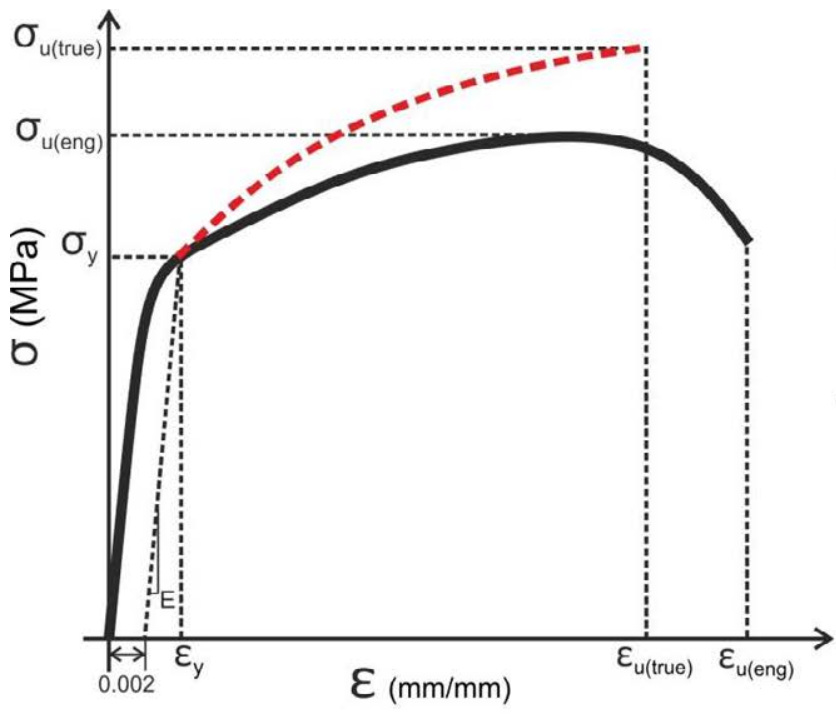

(a)

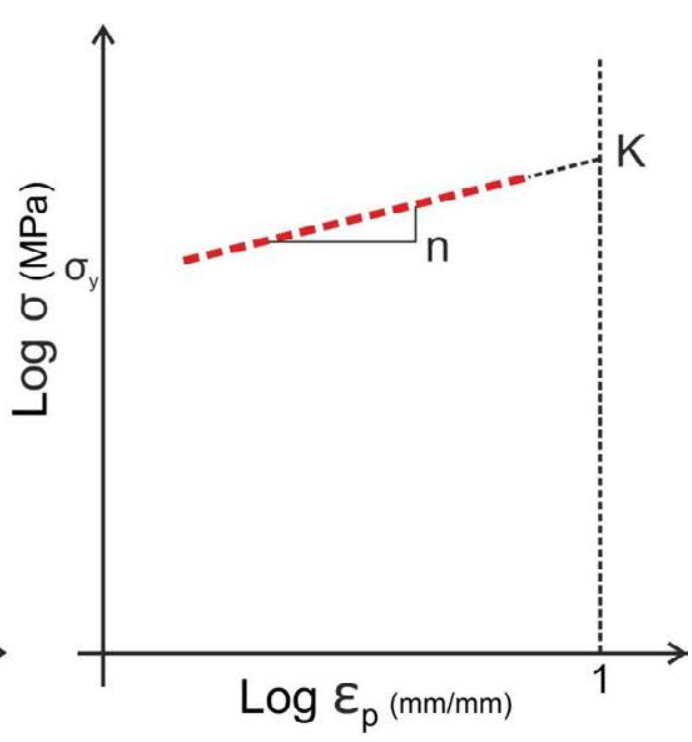

(b)

Figure 29 True and engineering stress-strain curves. (a) Stress versus strain curve in linear scale(b) stress versus plastic strain relation in Logarithmic scale.

The relations presented so far are valid provided that the load is applied monotonically. On the contrary, when a specimen is subjected to cyclic loads (i.e. fatigue loads), its stress-strain relation migth vary from that obtained through monotonic tensile tests.

If a component is repeatedly cycled with strain controlled loads, it will respond in one of the following manners: 
- Cyclic hardening (Figure 30, bottom): The stress developed in each successive strain reversal increases with the number of cycles applied.

- Cyclic softening (Figure 30, top): The stress decreases with the number of imposed cycles.

- Cyclically stable: The stress remains stable with the increase of the number of strain cycles.

- Mixed behavior: Might exhibit cyclic softening or hardening depending on the strain level.

This transient behavior is associated with the stability of the dislocations of the substructure in the crystal lattice of the material. Generally, soft and ductile materials will tend to cyclically harden whereas harder materials will tend to soften. Bannantines et al.(1990), proposed the following relation between yield and ultimate strength to determine the material's hardening or softening behavior:

$$
\begin{gathered}
\frac{\sigma_{u(\text { eng })}}{\sigma_{y}}>1.4 \Rightarrow \text { Hardening } \\
\frac{\sigma_{u(e n g)}}{\sigma_{y}}<1.2 \Rightarrow \text { Softening }
\end{gathered}
$$

For ratios between 1.2 and 1.4 the material usually exhibit stable or mixed behavior.

Cyclic hardening
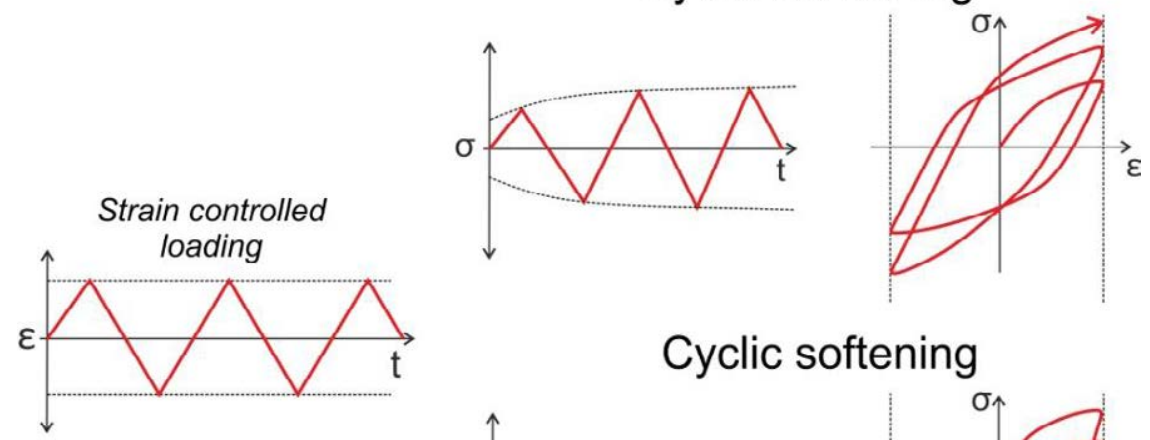

Cyclic softening
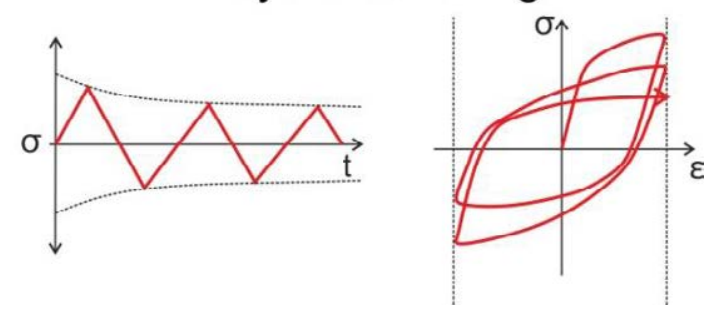

Figure 30 Cyclic hardening and softening behavior.

For both cyclic hardening and softening, the rate of stress change reduces gradually until reaching a stable state that will remain constant practically for the rest of the life of the test specimen. This stabilization usually takes place before the $10 \%$ of the total number of cycles to fatigue failure. 
The cyclic stable stress-strain hysteresis loop (usually taken at half of the fatigue life of the specimen) can be characterized by the parameters shown in Figure 31 (a). The peaks of the stable hysteresis loop peaks for various strain amplitudes will form the cyclic stress-strain curves (Figure 31 (b)).

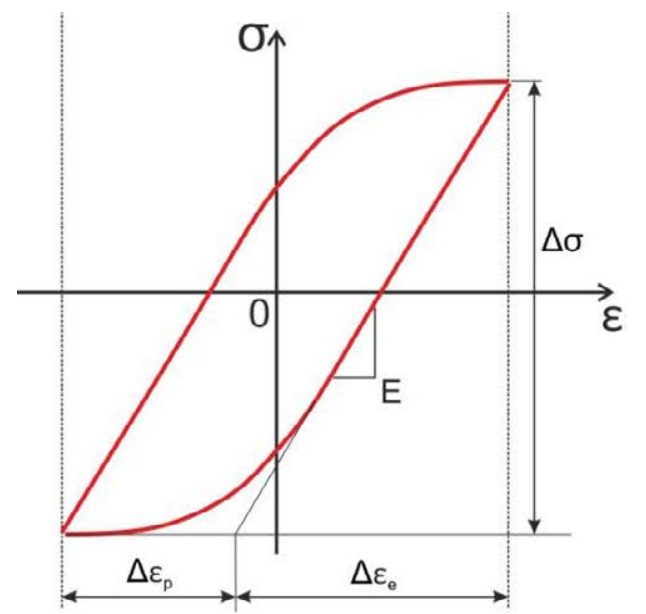

(a)

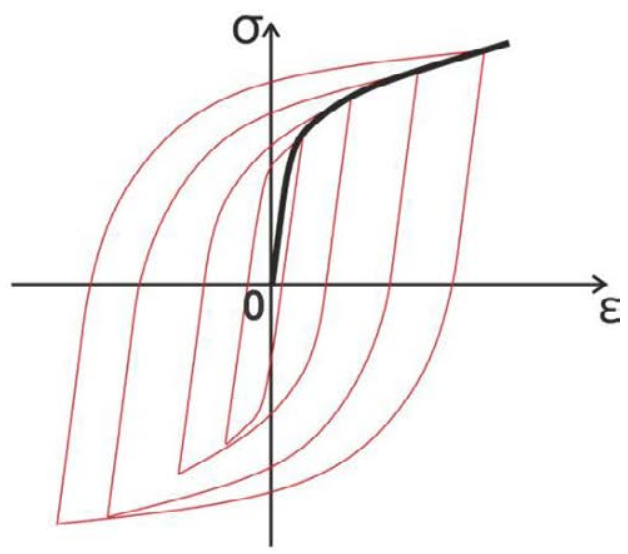

(b)

Figure 31 Cyclic stress-strain curve.

Similarly to monotonic curve, the stress-strain relation can be approximated by means of the following expression:

$$
\varepsilon=\varepsilon_{e}+\varepsilon_{p}=\frac{\sigma}{E}+\left(\frac{\sigma}{K^{\prime}}\right)^{1 / n^{\prime}}
$$

Where $n^{\prime}$ is the cyclic strain hardening exponent, and $K^{\prime}$ is the cyclic strength coefficient. It is observed on the expression that the elastic component of the strain curve remains equal with respect to the monotonic loading. On the other hand, the plastic straining behavior can be different between cyclic and monotonic loading. In Z. López et al. (2012), a complete work of correlation among cyclic and monotonic properties for a wide variety of steels can be found.

\subsubsection{DETERMINING STRAIN-LIFE CURVE FROM FATIGUE TEST DATA}

Four parameters need to be determined in order to fully characterize the strain-life behavior of a material according to eq. (1.7): fatigue strength coefficient $\left(\sigma_{f}^{\prime}\right)$, fatigue strength exponent $(b)$, fatigue ductility coefficient $\left(\varepsilon_{f}^{\prime}\right)$, and fatigue ductility exponent $(c)$. Although these parameters can be directly obtained by means of non linear least squares method (Langlays \& Vogel, 1996), it is usually preferred to use separate linear least squares fittings for both elastic and plastic strain amplitudes (Y.L. Lee et al., 2005). This approach enables to statistically emphasize the ductility and strength parameters separately by data discarding (C.R. Williams et al., 2003). The choice of the range of data for each curve depends in turn on range of the experimental data, and its positioning with respect to the transition fatigue life (eq. (1.8)): 
- If transition fatigue life lies within the experimental data range, this transition establishes a boundary for data separation. Due to the scatter nature of the fatigue behavior and the locations of the transition life point, the actual limit used for data separations becomes a subjective choice. By way of example, Williams proposed accounting any remaining data below the transition points and two data points immediately above it for ductility parameters analysis, and all life data points above and the two immediately below the transition life for strength parameters study.

- If transition fatigue life does not lie within the experimental data range, any data point with very low plastic strain (i.e. below $0.0005 \mathrm{~mm} / \mathrm{mm}$ approximately) should be discarded for ductility coefficients calculation. To determine the fatigue strength coefficients, all valid test data points can be accounted. It should be taken into account that data regression can show significant deviation as its curve moves away from the test range. Consequently, high plastic strain-life estimation obtained in this scenario should be treated with special care.

Both Basquin $\left(\varepsilon_{\mathrm{e}}-\mathrm{N}\right)$ and Coffin-Manson $\left(\varepsilon_{\mathrm{p}}-\mathrm{N}\right)$ are power relations and therefore can be approximated by the following linear relations in the logarithmic scale:

$$
Y=A+B \cdot X
$$

Where the values for each parameter for Basquin and Coffin-Manson relations are according to the following table:

Table 3: Linear regression parameters

\begin{tabular}{|c|c|c|c|c|}
\hline Equation & $\mathbf{X}$ & $\mathbf{Y}$ & $\mathbf{A}$ & $\mathbf{B}$ \\
\hline Basquin & $\log \left(\varepsilon_{e}\right)$ & $\log (2 N)$ & $(1 / b) \log \left(\sigma_{f}^{\prime}\right)$ & $1 / b$ \\
\hline Coffin-Manson & $\log \left(\varepsilon_{p}\right)$ & $\log (2 N)$ & $(1 / c) \log \left(\varepsilon_{f}^{\prime}\right)$ & $1 / c$ \\
\hline
\end{tabular}

The values of the best fit estimators for $A$ and $B$ can be obtained by least squares as follows:

$$
\begin{gathered}
\hat{A}=\bar{Y}-\hat{B} \cdot \bar{X} \\
\hat{B}=\frac{\sum_{i=1}^{n}\left(X_{i}-\bar{X}\right)\left(Y_{i}-\bar{Y}\right)}{\sum_{i=1}^{n}\left(X_{i}-\bar{X}\right)^{2}}
\end{gathered}
$$

Where: 


$$
\begin{gathered}
\bar{X}=\frac{1}{n} \sum_{i=1}^{n} X_{i} \\
\bar{Y}=\frac{1}{n} \sum_{i=1}^{n} Y_{i}
\end{gathered}
$$

In many occasions, fatigue designs are performed with a certain safety margin in order to ensure a probability of survival (i.e. $90 \%$ or $95 \%$ ) for a specific strain level. To this aim, the standard deviation can be very helpful to quantify the scatter of the test data with respect to the regression model, and thus establish confidence intervals:

$$
\hat{S}=\sqrt{\frac{\sum_{i=1}^{n}\left(Y_{i}-\hat{Y}_{i}\right)^{2}}{n}}
$$

An equivalent procedure can be conducted to obtain estimators for the parameters that define the plastic component of the cyclic Ramberg-Osgood equation (1.54), following the nomenclature correspondence of Table 4:

Table 4: Linear regression parameters for cyclic plastic strain-stress curve

\begin{tabular}{|c|c|c|c|c|}
\hline Equation & $\mathbf{X}$ & $\mathbf{Y}$ & $\mathbf{A}$ & $\mathbf{B}$ \\
\hline Basquin & $\log \left(\varepsilon_{p}\right)$ & $\log (\sigma)$ & $\log \left(K^{\prime}\right)$ & $n^{\prime}$ \\
\hline
\end{tabular}

Equating Basquin and Coffin-Manson relations to the elastic and plastic component of Ramberg-Osgood equation respectively, the following compatibility relations can be established:

$$
\begin{array}{r}
K^{\prime}=\frac{\sigma_{f}^{\prime}}{\left(\varepsilon_{f}^{\prime}\right)^{n^{\prime}}} \\
n^{\prime}=\frac{b}{c}
\end{array}
$$

It should be noticed that the parameters of these relations have been obtained by means of curve fittings, so these equities will be valid only in the case of ideal perfect fit regressions $(\hat{S}=0)$. Nonetheless, these compatibly equations suppose a suitable indicator of the regression fits quality.

Finally, if no fatigue experimental data is available, the four strain to life parameters can be approximated from monotonic mechanical properties of the material. In this regard, V.T. 
Troshchenko \& L.A. Khamaza (2010) bring a wide assessment of the most relevant methods proposed.

\subsubsection{STRAIN CONCENTRATION BEHAVIOR ON WELDED JUNCTIONS}

The assembly process of the rectangular tube beams that compound the LPTV's structures is carried out by means of various welding techniques. Most of these techniques imply the utilization of filling material that will protrude from the jointed material once the welding operation is finished (i.e. weld bead). It supposes a steep change in the geometry of the junction that will lead to a strain raise at the toe of the aforementioned weld bead.

The analysis of these local notches is usually performed by means of the stress $\left(K_{\sigma}\right)$ or strain $\left(K_{\varepsilon}\right)$ concentration factors, which reads as the ratio between the maximum local stress or strain and the nominal one (i.e. without influence of any local effect).

$$
\begin{gathered}
K_{\sigma}=\frac{\sigma_{\max }}{\sigma_{\text {nom }}} \\
K_{\varepsilon}=\frac{\varepsilon_{\max }}{\varepsilon_{\text {nom }}}
\end{gathered}
$$

The evolution of these parameters with respect to the local maximum stress is shown in Figure 32. It is noticed that both $K_{\sigma}$ and $K_{\varepsilon}$ remain equal and constant in the linear range of material stress strain curve. After local yielding the material flow curve is no longer linear, modifying the concentration behavior. In this local plastification situation, $K_{\varepsilon}$ tends to increase as $K_{\sigma}$ tends to decrease.

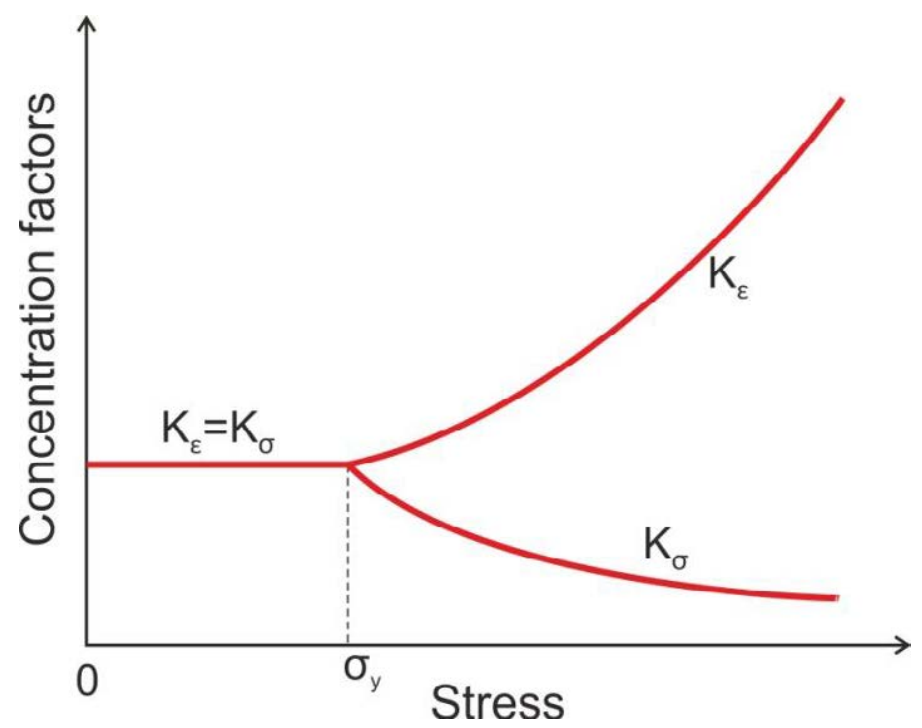

Figure 32 Concentration factors evolution. 
While the influence of nonlinear behavior of the stress and strain concentrations may have moderate influence in HCF, it might be significant for LCF due to the plastic deformation present in this fatigue life range.

A wide range of approaches have been carried out with the aim of quantifying the stress or strain concentration factors of different materials, which can be classified in the following methods:

- Parametric equations to relate nominal to local maximum stresses and strains. This procedure is probably the least time consuming option, but has the drawback that the proposed equations have limited application and accuracy. In this regard, of special relevance are the contributions of H. Neuber (1961) or G. Glinka \& A. Newport (1987).

- Finite element analyses. This theoretical approach implies extend knowledge of the geometrical shape that is producing the stress or strain raise, and its accurate modeling and meshing (W. Fricke, 2008), which entails heavy models that are in many occasions time and cost consuming to solve. Additionally, when non linear behavior must be accounted, the analysis becomes nonlinear as well, introducing a new source of complexity to the solving process.

- Experimental methods. In most of the cases suppose the most costly approach, but if the experiment setup and the data processing is correct, it is possible to obtain the most accurate results with the least previous knowledge of the component in study. The most extended technique is the use of strain gauges (F.R. Mashiri et al., 2004, W.M. Gho \& F. Gao, 2004), although it presents several drawbacks. First, they cannot be installed at geometric discontinuities (i.e. weld toe), compelling the use of extrapolation techniques to estimate actual maximum local strain. Second, due to the nature of the weld geometry, it is not possible to know in advance the exact location of the maximum strains, and inaccurate installation of the strain gauges inevitably leads to an erroneous prediction of the SCF. An alternative technique capable to overcome these limitations, known as Digital Image Correlation (DIC, M. Sutton et al., 2009), was preferred for the experimental analysis of the present thesis.

A more complete description of DIC technique and its potential for measuring strain concentrations in welded junctions is presented in the next subsection

\subsubsection{DIC for measuring strain concentrations in welded junctions}

Digital Image Correlation can be defined as a non contact optical technique that acquires, stores and performs image analysis to extract full field shape, displacements and strains. The displacement calculation procedure of DIC from unloaded and loaded images respectively is schematized in Figure 33. 


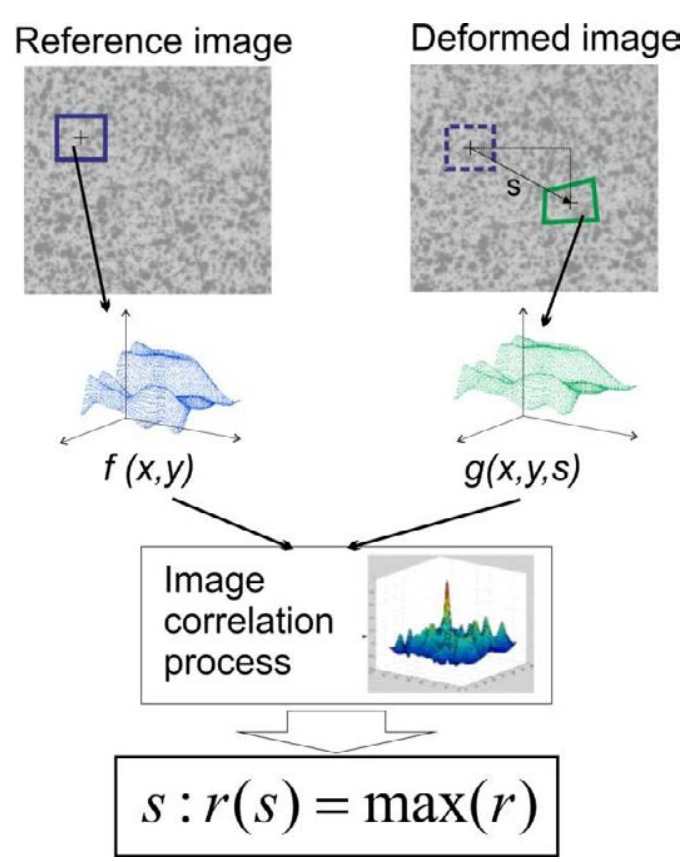

Figure 33 Image correlation schema.

First, the unloaded or reference image is divided into small squares formed by $2 N+1 \times 2 N+1$ pixels called subsets. Then, a grayscale function $(f(x, y))$ is constructed for each subset by means of bilinear or bicubic interpolators. On the basis that the subset position in the reference and deformed images are related via a transformation function $(f(x, y)=g(x, y, s))$, an iterative correlation process starts with the purpose of determining the parameters vector ' $s$ ' with the highest accuracy. This vector contains the information of the subset displacements $(u, v)$. The type of the transformation function employed for the correlation process will mark the size of the parameter vector ' $s$ ' and also the degree of deformation that can be accounted during the correlation process (Figure 34).

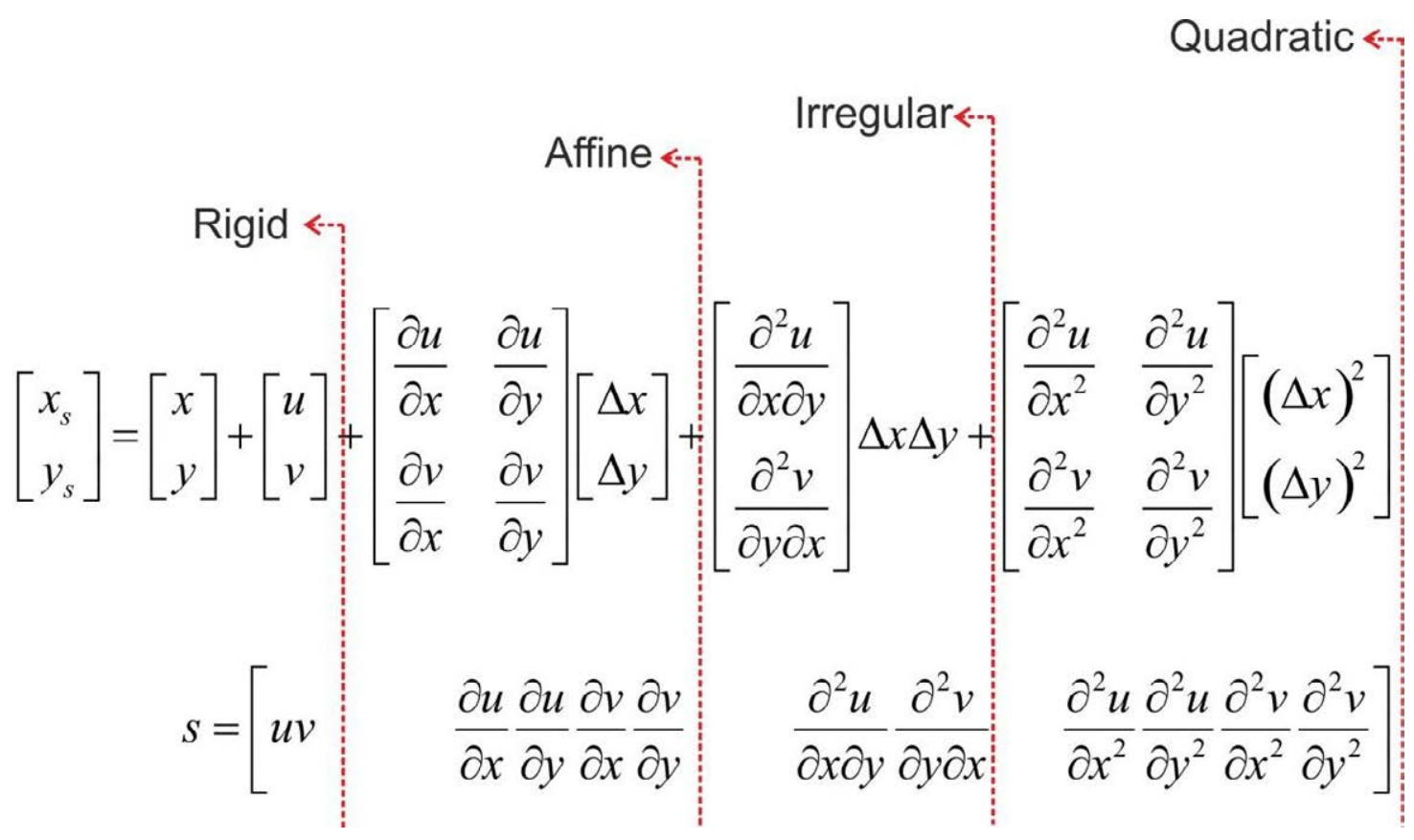

Figure 34 DIC Transformation functions. 
The iterative correlation process will halt once the desired similarity between reference and deformed image is reached. This similarity is typically measured by means of correlation functions such as the cross-correlation coefficient $r_{\mathrm{CC}}$ (eq. ) and the sum-of-squared-differences correlation coefficient $r_{\text {SSD }}$ (eq.) (P. Lava et al.,2009).

$$
\begin{aligned}
& \frac{\sum_{y=s c-N} \sum_{x=s c-N}^{s c+N} f \cdot g}{\left.\sum_{x=s c-N}^{s c+N} f^{2}\right)\left(\sum_{y=s c-N}^{s c+N} \sum_{x=s c-N}^{s c+N} g^{2}\right)} \\
& \sum^{s c+N} \sum^{s c+N}(f-g)^{2} \\
& r_{S S D}=\frac{y=s c-N x=s c-N}{\sqrt{\left(\sum_{y=s c-N}^{s c+N} \sum_{x=s c-N}^{s c+N} f^{2}\right)}}
\end{aligned}
$$

Where the subscript 'sc' refers to the subset center.

Once the correlation function is maximized, and the displacements obtained, the strains can be determined by means of the post-processing operation summarized in Figure 35.

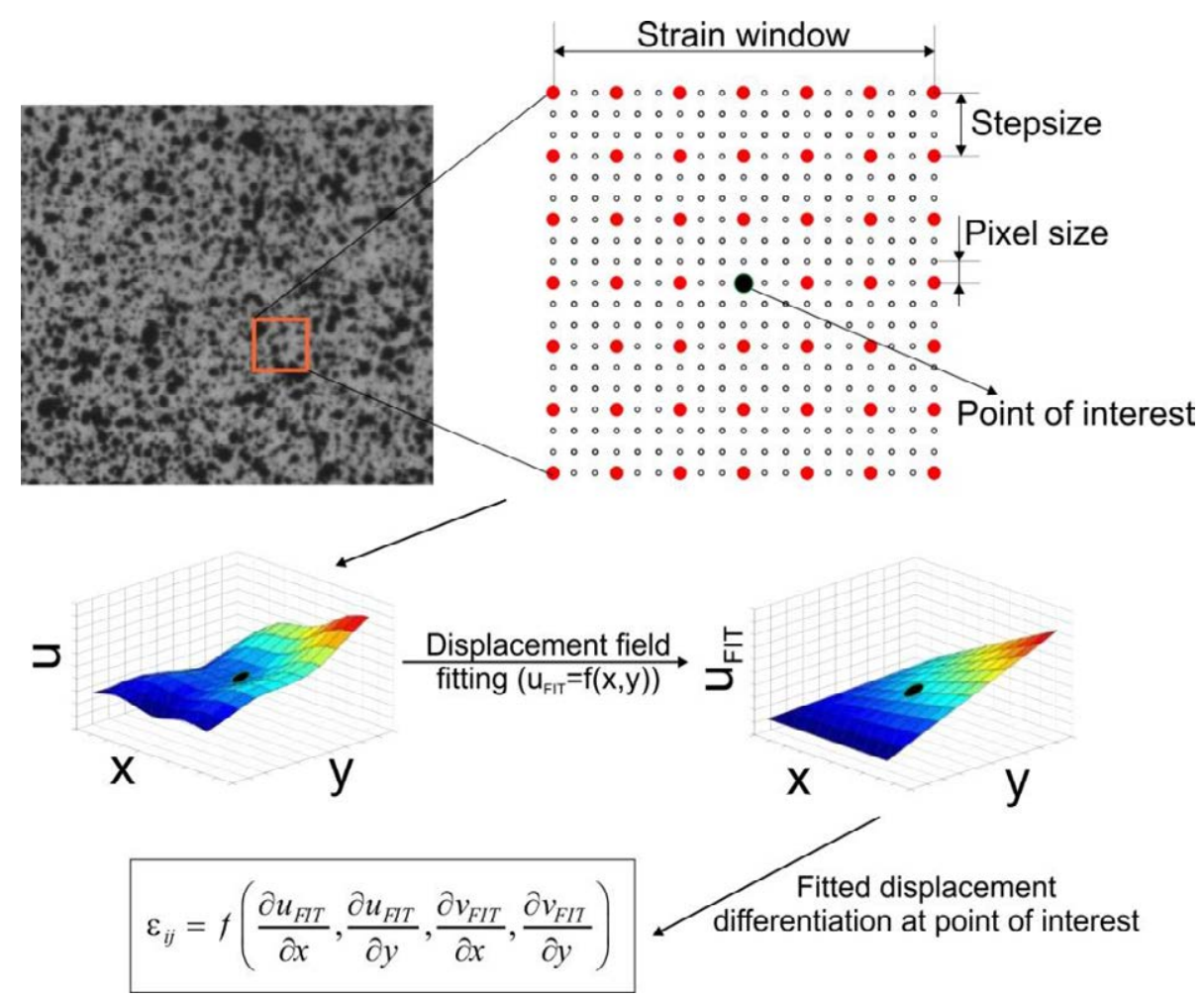

Figure 35 DIC strain calculation procedure. 
To obtain the strains on a point of the image, a square strain window (SW) containing $N x N$ data points in its vicinity is selected. Then, the previously obtained displacements from the DIC process at these data points are fitted using first or second order Lagrange polynomials. For the case of ' $u$ ' displacements:

$$
u_{F I T(x, y)}=\sum_{i=0}^{n} \sum_{j=0}^{n} a_{i j} x^{i} y^{j}
$$

If $n=1$, the expression will allow the strain window to deform into an irregular quadrangle, while second order polynomial $(n=2)$ will also account for quadratic deformation over this region. The parameters values ' $a_{i j}{ }^{\prime}$ of the fit functions are determined by minimizing the least squares differences between actual and fitted displacement values:

$$
S_{(x, y)}=\sum_{i=0}^{N^{2}}\left(u_{D I C}-u_{F I T}\right)^{2}
$$

Once the Lagrange polynomials coefficients are determined, the deformation gradient can be easily obtained by differentiation of the displacement fit functions $u_{F I T}$ and $v_{F I T}$ :

$$
F=\left[\begin{array}{cc}
1+\frac{\partial u_{F I T}}{\partial x} & \frac{\partial u_{F I T}}{\partial y} \\
\frac{\partial v_{F I T}}{\partial x} & 1+\frac{\partial v_{F I T}}{\partial y}
\end{array}\right\rfloor
$$

Finally, the strains can be obtained by from the deformation gradient using any strain tensor expression. Most commonly used strain tensors in DIC are Euler-Almansi (eq. (1.70)) and Green-Lagrange (eq.(1.71)).

$$
\begin{aligned}
& \varepsilon^{\ln E A}=\ln \left(F \cdot F^{T}\right)^{0.5} \\
& \varepsilon^{G L}=\frac{1}{2}\left(F^{T} \cdot F-I\right)
\end{aligned}
$$

From the parameters shown in Figure 35, it is easy to determine the size of the so-called Virtual Strain Gauge (VSG):

$$
V S G(m m)=[(S W-1) S s+1] P s
$$

Where $S W$ refers to the strain window, $S s$ to the step size and $P S$ to the pixel size. The larger the VSG size is, the less noisy strain fields will be obtained. On the contrary, since the 
displacement surface fit is limited to a second order polynomial, it will not be able to adapt correctly to heterogeneous displacement fields within the SW area.

Furthermore, is possible to relate the smoothing error with the VSG for heterogeneous strain fields due to local strain raising on welded junctions as it was proven in (J. Pérez et al., 2016). To do so, the strain calculation process previously described was implemented over results obtained from FEM models of 120 different weld bead shapes. This allows eliminating any other source of noise that might arise during image acquisition and/or processing, thus isolating the smoothing error.

In order to study the smoothing error in a consistent manner, it is important to keep the same pixel size (in our case, distance between data points or nodes), so that the VSG is also constant along the profile. This mimics the unrealistic situation of a single camera always perpendicularly aligned to the weld profile. To do so, the displacement profile was not extracted directly from FEM results, but obtained by discrete integration of the FEM strain profile as described in Figure 36.

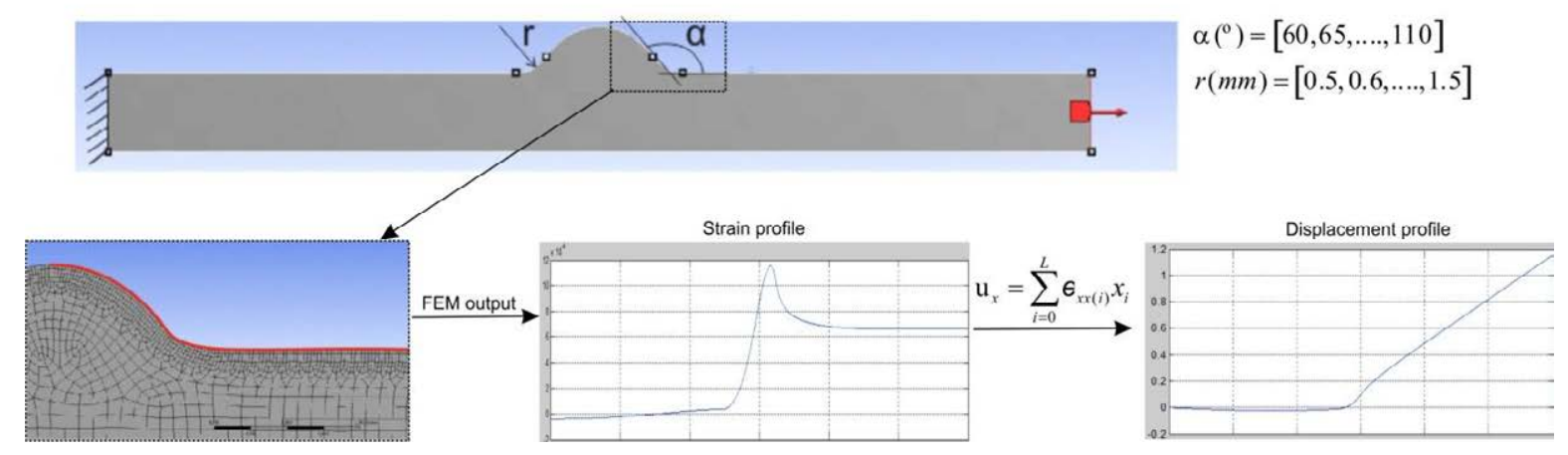

Figure 36 Theoretical SCF obtained with FE simulations (J. Pérez et al., 2016).

Once all displacement profiles were obtained, the post-processing process was applied to calculate the strains using VSG sizes from 0.1 to $2.3 \mathrm{~mm}$ for each of the 120 profiles. Afterwards, the relative smoothing error was obtained as the difference between peak strains obtained from FEM models and from the DIC processing:

$$
\text { Relative error }(\%)=\frac{\max \left(\varepsilon_{F E M}\right)-\max \left(\varepsilon_{C A L}\right)}{\max \left(\varepsilon_{F E M}\right)}
$$

The results obtained are shown in Figure 37. There can be distinguished vertical groups of 120 blue dots for each VSG size studied, each group belonging to a different weld profile geometry. The red line represents the mean error, and the black ones the mean error plus/minus one standard deviation.

As expected from the theoretical considerations, the smoothing error increases when increasing the VSG size. The maximum error remains negligible for VSG under approximately 0.3 $\mathrm{mm}$ regardless the weld shape. It is also observed that the relative error increasingly scatters with increasing VSG, evidencing more significant influence of the weld bead geometry for larger VSG sizes. 
In any case, deviations do not increase dramatically with the VSG, keeping the mean error around $15 \%$ for a VSG size of $2 \mathrm{~mm}$, not very difficult to achieve in normal conditions.

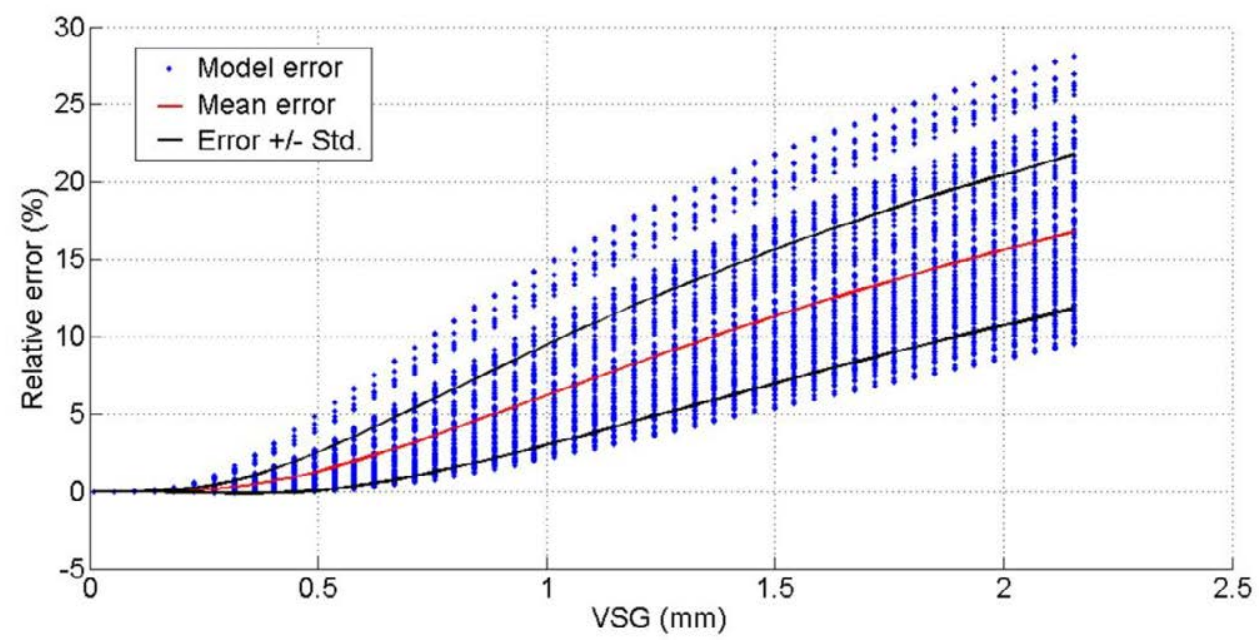

Figure 37 Relative smoothing error (J. Pérez et al., 2016). 


\section{DYNAMIC LOAD CYCLES ON LPTV STRUCTURES DURING OPERATION}

The direct impact of the input service loads on the fatigue analysis results have been evidenced in the theoretical background of previous sections. In some occasions, these loads can be easily calculated or measured. In other cases, however, these loads cannot be accurately known in advance, and extensive data acquisition and processing become necessary for a correct approach.

In the case of LPTV operation loads, it is common to base structural analysis on high severity specific load cases usually based on dynamic limits of the vehicle (J.P. Wideberg, 2004; B. Gombor, 2005). Although this approach may be appropriate to ensure vehicle's integrity under these extreme events, for fatigue studies a characterization of the actual operating loads takes on greater importance, since most likely they will be the cause structural failure, and not the high severely, least frequent ones. Therefore, representative data acquisition, and posterior determination of load cycles become necessary tasks to obtain reliable fatigue results.

LPTV's dynamic loads can be divided into longitudinal (acceleration and braking), lateral (turning) and vertical (road imperfections and slope changes). The first two occur at such frequencies that allow its characterization by means of GPS positioning data differentiation as will be shown later (J.A. Perez et al., 2014). Vertical accelerations, on the contrary, arise primarily from road imperfections, that produce a low amplitude and high frequency response, difficult to detected with common GPS equipments. For this reason, a portable acquisition system based on a triaxial accelerometer was designed to record these accelerations at a acquisition rate of $100 \mathrm{~Hz}$.

The subsequent sections present the procedure for obtaining the LPTV's acceleration time series from the two methods aforementioned. Later, the resulting load cycles obtained from the application of the 'Rainflow' counting cycle method are presented.

\subsection{LATERAL AND LONGITUDINAL OPERATING LOADS FROM GPS POSITIONING DATA}

As stated before, instant position data obtained from a Global Positioning System (GPS) was used to characterize lateral and longitudinal operating accelerations on LPTV's structures. To ensure the representativeness of the sample, data processing was performed out of GPS recordings coming from 10 different LPTV models belonging to the Empresa Municipal de Transportes de Madrid (EMT), monitored during an operating time of more than 600 hours over 13 different urban routes. These data were acquired by INSIA using a cinematic recording equipment under the project Ecotram (2009). The GPS acquisition rate was $1 \mathrm{~Hz}$.

The methodology for extracting the lateral and longitudinal accelerations was based on GPS recordings referred to World Geodetic System 1984, so the first step was to convert the east and north directions to instantaneous positions $\left(X_{i} Y_{i}\right)$ according to the Universal Transverse 
Mercator (UTM) coordinates. The algorithm employed to perform this conversion was developed by the United States Geological Survey (USGS), considering the earth as and ellipsoid with the following principal parameters:

Table 5: WGS84 ellipsoid parameters

\begin{tabular}{|c|c|c|c|c|}
\hline $\begin{array}{c}\text { Equatorial radius } \\
(\boldsymbol{a})\end{array}$ & Flattening (f) & Eccentricity $(\boldsymbol{e})$ & Polar radius (b) & $\begin{array}{c}\text { Longitude } \\
\text { origin }\left(\boldsymbol{\lambda}_{\boldsymbol{o}}\right)\end{array}$ \\
\hline 6378137 & $1 / 298.25722$ & 0.08181919 & 6399593.626 & 0.0523598 \\
\hline
\end{tabular}

From these parameters, a series of constants can be determined, which will be later the core of the final equation for UTM coordinates conversion. Given $\lambda$ and $\phi$ the longitude and latitude positions respectively:

$$
\begin{aligned}
& e^{\prime}=\frac{e^{2}}{1-e^{2}} \\
& N=\frac{a}{\sqrt{\left(1-e^{1^{2}} \cdot \sin ^{2}(\varphi)\right)}} \\
& T=\tan ^{2}(\varphi) \\
& C=e^{\prime} \cdot \cos ^{2}(\varphi) \\
& A=\cos (\varphi)\left(\lambda-\lambda_{0}\right) \\
& M=a \cdot\left[\begin{array}{l}
\left(1-\frac{e^{\prime 2}}{4}-\frac{3}{64}\left(e^{\prime^{2}}\right)^{2}-\frac{5}{256}\left(e^{\prime^{2}}\right)^{3}\right) \cdot \varphi- \\
-\left(\frac{3}{8} \cdot e^{1^{2}}+\frac{3}{32} \cdot\left(e^{\prime 2}\right)^{2}+\frac{45}{1024} \cdot\left(e^{\prime 2}\right)^{3}\right) \cdot \sin (2 \varphi)+ \\
+\left(\frac{15}{256}\left(e^{1^{2}}\right)^{2}+\frac{45}{1024}\left(e^{1^{2}}\right)^{3}\right) \cdot \sin (4 \varphi)- \\
-\left(\frac{35}{3072}\left(e^{1^{2}}\right)^{3}\right) \cdot \sin (6 \varphi)
\end{array}\right]
\end{aligned}
$$


Finally, with the use of these constants, the UTM $\left(X_{i} Y_{i}\right)$ positions can be obtained by means of the following expressions:

$$
\begin{aligned}
& U T M_{-} X=0.9996 \cdot N \cdot\left(A+\frac{(1-T+C) \cdot A^{3}}{6}+\frac{\left(5-18 \cdot T+T^{2}+72 \cdot C-58 \cdot e^{12}\right) \cdot A^{3}}{120}\right)+500000 \\
& U T M_{-} Y=0.9996 \cdot\left(M+N \cdot \tan (\varphi) \cdot\left(\begin{array}{l}
\frac{A^{2}}{2}+\frac{\left(5-T+9 \cdot C+4 \cdot C^{2}\right) \cdot A^{4}}{24}+ \\
+\frac{\left(61-58 \cdot T+T^{2}+600 \cdot C-330 \cdot e^{\prime 2}\right) \cdot A^{6}}{720}
\end{array}\right)\right)
\end{aligned}
$$

Once all recorded data have been converted, it is possible to reconstruct the LPTV journey and thus check the correctness of the conversion. By way of example, the following Figure 38 (J. Perez et al. 2014) shows the path of the $L 63$ route.

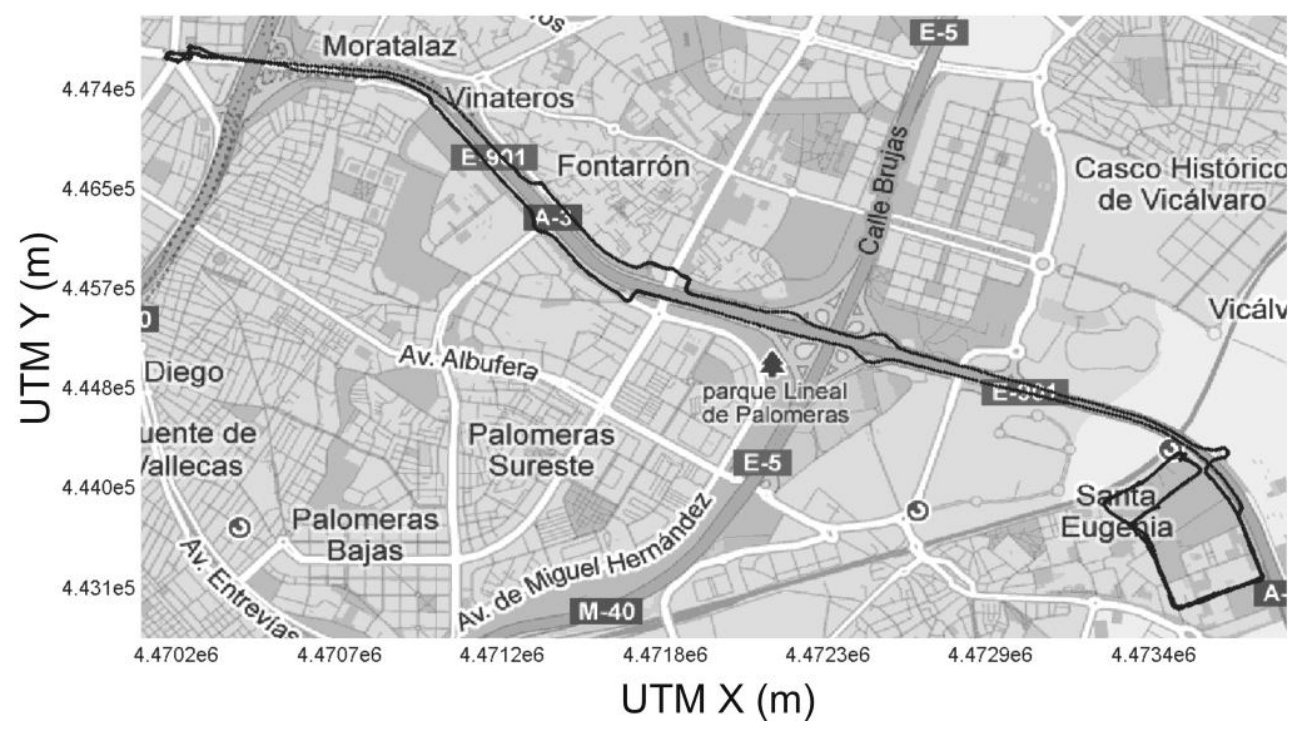

Figure 38 Example of urban route L63 in Madrid.

From the obtained instantaneous positions, longitudinal accelerations can be obtained using second order central differentiation (A.M. Bruton et al. 1999). According to this method, velocity is first obtained according to the equation:

$$
V_{i}=\frac{\frac{P_{i+1}-P_{i}}{\Delta t}+\frac{P_{i}-P_{i-1}}{\Delta t}}{2}=\frac{P_{i+1}-P_{i-1}}{2 \Delta t}
$$

Where $P_{i-1}=\left(X_{i-1}, Y_{i-1}\right), P_{i}=\left(X_{i}, Y_{i}\right)$ and $P_{i+1}=\left(X_{i+1}, Y_{i+1}\right)$, are the position vectors at $i-1, i$ and $i+1$ instants respectively, and $\Delta t$ is the increment of time between two recordings (i.e. 1 second). Results of eq.(1.82) are then differentiated again to obtain the instant accelerations values as following: 


$$
a_{\text {long }(i)}=\frac{V_{i+1}-V_{i-1}}{2 \Delta t}=\frac{P_{i+2}-2 P_{i}+P_{i-2}}{4 \Delta t^{2}}
$$

For the lateral accelerations, is it well know that they are related to the velocity and turning radius $\left(R_{i}\right)$ as follows:

$$
a_{\text {lat }(i)}=\frac{V_{i}^{2}}{R_{i}}
$$

Where $R_{i}$ is determined using the equation of a circumference that passes through three points $\left(\left(X_{i-1}, Y_{i-1}\right)\left(X_{i}, Y_{i}\right),\left(X_{i+1}, Y_{i+1}\right)\right)$ which writes as eq. (1.85) for a circumference of center at $(a, b)$ and radius $R$.

$$
X_{(t)}^{2}+Y_{(t)}^{2}+D X_{(t)}+E Y_{(t)}+F=0
$$

Substituting $X_{(t)}$ and $Y_{(t)}$ of eq.(1.85) by the actual positions at instants $i-1, i$ and $i+1$ it can be constructed a three equation system with three unknowns $(D, E, F)$. Once solved, the instantaneous circumference center and radius of curvature is obtained as follows:

$$
\begin{gathered}
a_{(i)}=-\frac{D}{2} \\
b_{(i)}=-\frac{E}{2} \\
R_{(i)}=\sqrt{a^{2}+b^{2}-F}
\end{gathered}
$$

Finally, by substituting eq.(1.82) in eq.(1.84) we can construct the expression for obtaining lateral accelerations as a function of the instant position and turning radius:

$$
a_{\text {lat }(i)}=\frac{\left(P_{i+1}-P_{i-1}\right)^{2}}{4 \Delta t^{2} R_{i}}
$$

Taking equations (1.83) and (1.89), it is possible to determine the time series for the longitudinal and lateral accelerations respectively. The following Figure 39 (J. Pérez et al. 2014) shows, as an example, accelerations obtained for a randomly taken period of 1000 seconds. 

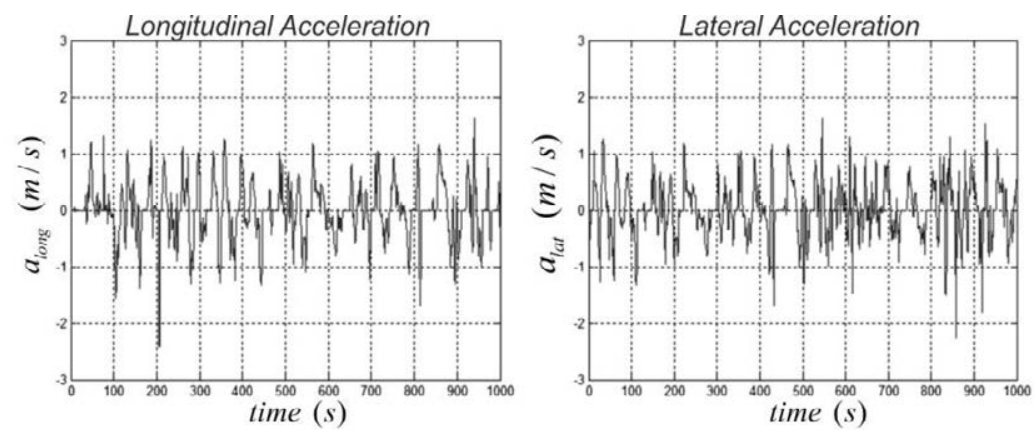

Figure 39 Example of longitudinal and lateral acceleration time series.

From the overall time series obtained, it is possible to evaluate the acceleration state, that is, the relation between lateral and longitudinal acceleration at each instant. This relation is shown in Figure 40, where a cross shape relation can be observed. This shape evidences that significant accelerations do not take place in a combined manner. For example, if a LPTV is taking a curve that supposes significant lateral acceleration, it is very unlikely that it breaks or accelerates significantly at the same time. Analogously, important brake or acceleration maneuvers are most likely to occur on straight stretches rather than in curves. This evidence goes in good accordance with the common driving habits that ensure passengers conformability.

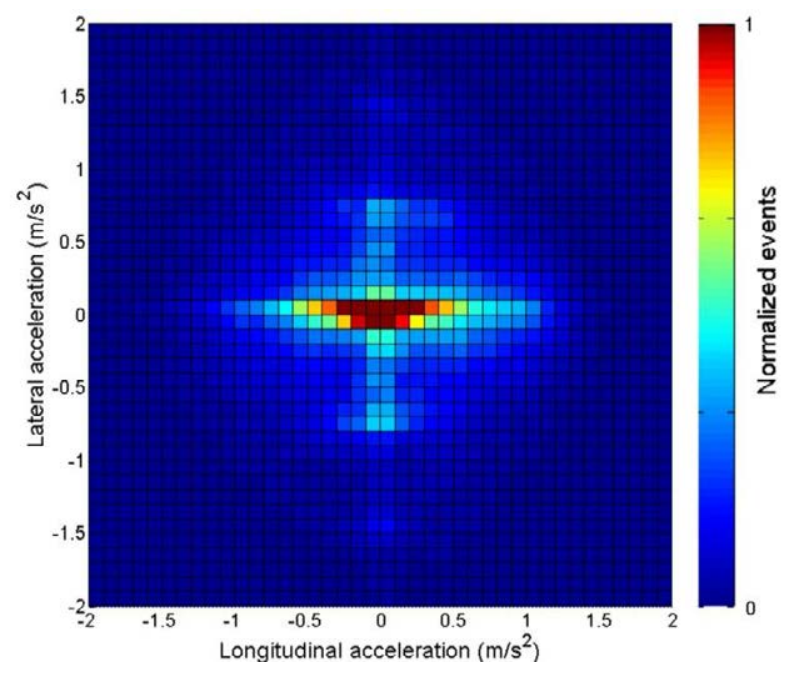

Figure $\mathbf{4 0}$ Lateral versus longitudinal acceleration.

Regarding the precision of the process, an extensive study of the measurement quality was carried out in J. Perez et al. 2014. It used the GPS positioning errors ' $\varepsilon$ ' (Figure 41) to calculate the consequent acceleration errors in the most unfavorable situations shown in Figure 42 and Figure 43. For longitudinal accelerations, it was evaluated the conservative assumption that every point contained in the angles $\alpha= \pm 20$ and $\alpha=180 \pm 20^{\circ}$ will cause the same error as if it were in the direction of movement. Similarly, every point contained in the angles $\alpha=90 \div \pm 20$ ㅇ and $\alpha=90^{\circ} \pm 20^{\circ}$ was supposed to cause the same error for lateral accelerations as if it were in the perpendicular to the movement at instant ' $i$ '. 


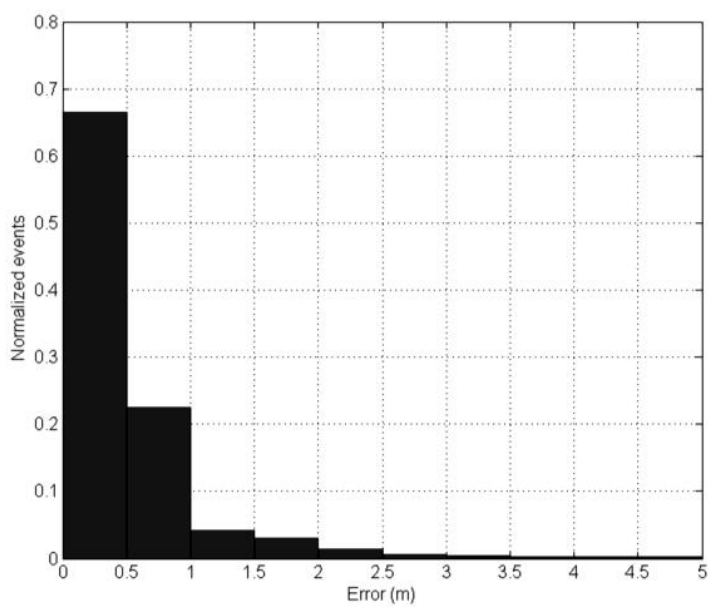

Figure 41 Positioning deviation (J. Perez et al., 2014).

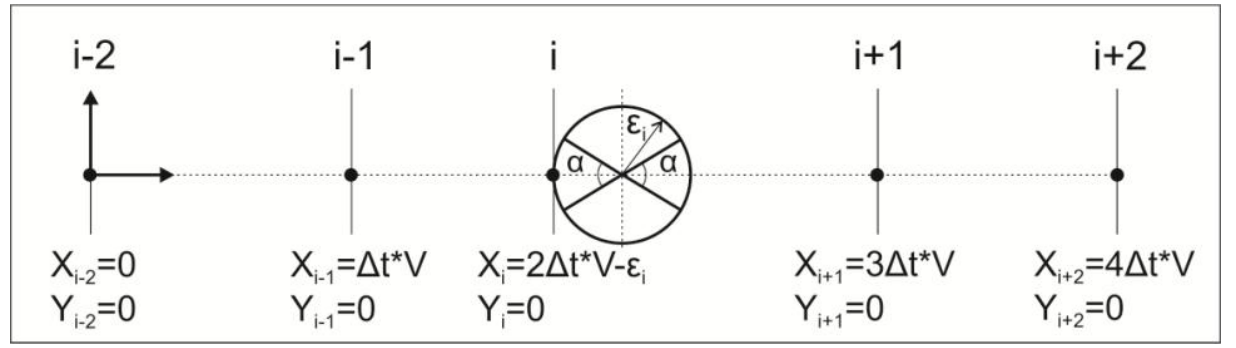

Figure 42 Most unfavorable error case for longitudinal accelerations (J. Perez et al., 2014).

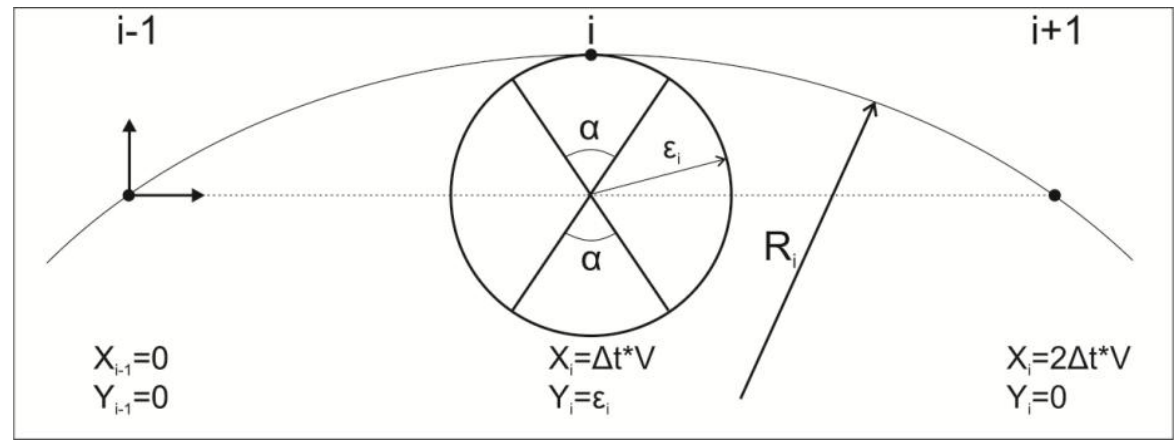

Figure 43 Most unfavorable error case for lateral accelerations (J. Perez et al., 2014).

Applying the second order central differentiation method to these specific cases situations it is possible calculate the longitudinal and lateral accelerations due to positions errors as follows:

$$
\begin{aligned}
& \varepsilon_{a_{\text {long }}(i)}=\frac{P_{i+2}-2 P_{i}+P_{i-2}}{4 \Delta t^{2}}=\frac{4 \Delta t V-2\left(2 \Delta t V-\varepsilon_{i}\right)+0}{4 \Delta t^{2}}=\frac{1}{2} \varepsilon_{i} \\
& \varepsilon_{a_{\text {lat }(i)}}=\frac{\left(P_{i+1}-P_{i-1}\right)^{2}}{4 \Delta t^{2} R_{i}}=\frac{\left(\sqrt{(2 \Delta t V-0)^{2}+(0-0)^{2}}\right)^{2}}{4 \Delta t^{2} R_{i}}=\frac{4 \Delta t^{2} V^{2}}{4 \Delta t^{2} R_{i}}=\frac{V^{2}}{R_{i}}
\end{aligned}
$$

The radius ' $R i$ ' can be expressed as follows in this situation: 


$$
R_{i}^{2}=V^{2}+\left(R_{i}-\varepsilon_{i}\right)^{2} \Rightarrow R_{i}=\frac{V^{2}+\varepsilon_{i}^{2}}{2 \varepsilon_{i}}
$$

Finally, substituting eq.(1.92) in eq.(1.91) it is obtained the expression (1.93) for the lateral accelerations error. It can be noticed that the term $\left(\varepsilon^{2} / \mathrm{V}^{2}\right)$ tends towards zero as the vehicle's velocity increases, becoming practically independent of the velocity for vehicle speeds over $5 \mathrm{~km} / \mathrm{h}$.

$$
\varepsilon_{a_{\text {lat }(i)}}=\frac{2 V^{2} \varepsilon_{i}}{V^{2}+\varepsilon_{i}^{2}}=\frac{2 \varepsilon_{i}}{1+\frac{\varepsilon_{i}^{2}}{V^{2}}} \approx 2 \varepsilon_{i}(\forall V>5(\mathrm{~km} / \mathrm{h}))
$$

Using equations (1.90) and (1.93) it is possible to calculate the acceleration error due to positioning uncertainty of the GPS system for longitudinal and lateral accelerations respectively. The values obtained for confidence levels of $90 \%$ and $95 \%$ and vehicle speeds $>5 \mathrm{~km} / \mathrm{h}$ are summarized in Table 6. As expected from the equations, the lateral acceleration errors are approximately four times the longitudinal acceleration errors.

Table 6: Acceleration uncertainty due to the GPS positioning error

\begin{tabular}{|c|c|c|}
\hline Confidence & $\begin{array}{c}\text { Lateral } \\
\text { acceleration }\end{array}$ & $\begin{array}{c}\text { Longitudinal } \\
\text { acceleration }\end{array}$ \\
\hline $90 \%$ & 0.024 & 0.006 \\
\hline $95 \%$ & 0.28 & 0.071 \\
\hline
\end{tabular}

Beyond this evaluation, a procedure to remove recordings that might come from erroneous positioning on the GPS system was carried out. The criteria used was based in the dynamic constrains of such type of vehicles and are the following:

- Recordings with longitudinal acceleration greater than $6 \mathrm{~m} / \mathrm{s}^{2}$. This limit was set based on test data produced by INSIA on the maximum acceleration attainable from equivalent vehicles.

- Recordings with decelerations greater than $10 \mathrm{~m} / \mathrm{s}^{2}$, which is the maximum possible value in a emergency braking over a high adhesive pavement.

- Recorded velocities above the maximum for an LPTV. A conservative value of 100 $\mathrm{km} / \mathrm{h}$ was taken even though most of these vehicles do not exceed $80 \mathrm{~km} / \mathrm{h}$.

- Data with turning circles under $4 \mathrm{~m}$. To attain this turning circle, a very short vehicle ( $4 \mathrm{~m}$ wheelbase) would need to turn the inner wheel over 60 .

- Recordings with lateral accelerations greater than $8 \mathrm{~m} / \mathrm{s}$, value that would make any vehicle of these characteristics overturn. 
As it can be observed, these limits where established on the basis that they are either physically impossible, or would have led to dynamic instability and thus to accidents, and none of these situations took place during the recorded period.

\subsection{VERTICAL OPERATING LOADS FROM ACCELEROMETER BASED ADQUISITION SYSTEM}

As previously stated, the source of vertical accelerations differs from those of the lateral and longitudinal ones since they are not originated during operation maneuvers, but from road imperfections. The principal consequence of this difference is the frequency at which they occur, which will in turn depend on the bump characteristics, and the LPTV passing velocity. In any case, vertical accelerations occur at higher frequencies than lateral and longitudinal ones, and they cannot be characterized on GPS based equipments. An acquisition system capable of recording at higher rates is thus required to accurately measure vertical accelerations.

On the other hand, accelerations should be measured during normal operation of the LPTV in order to obtain a representative sample. It compels to construct a least intrusive acquisition system that has no influence on the bus operation. It was also required by the EMT company to use a discreet acquisition system that does not disturb or attract the passengers attention.

For these reasons, it was decided to construct an ad-hoc acquisition system that meets the requirements (Figure 1). It consists of the following components:

- Triaxial accelerometer MMA7361: capacity micro-machined accelerometer, acquires the accelerations and sends them to the processor unit in form of milivolts.

- Microcontroller: contains the instructions of the acquisition process, it controls the accelerometer configurations, and sends the acquired data to a storage unit. Also supplies the necessary power to these devices.

- Micro SD storage unit: saves raw acceleration data in a 4 Gigabyte micro SD card.

- Battery: supplies the power to the microcontroller, which then distributes to the accelerometer and storage unit.

- Connection board: power supply and ground connection were made using this connection board.

The following Table 7 shows the most important acquisition characteristics. The micro controller has an ADC converter with 10 bit resolution, which means that the analog signal input is divided in $2^{10}$ discrete digital values over the voltage range (i.e. 3.3 Volts). Consequently, the minimum voltage change necessary to change the digital converted signal by one division (or level) is of 0.0032 volts. On the other hand, the accelerometer response changes at the rate of 0.8 Volts per g. Dividing this sensitivity value by the resolution by the microcontroller, the resolution of the measure is obtained as the acceleration change needed to change the digital converted signal by one division. It results on $0.004 \mathrm{~g}$ (or $0.0395 \mathrm{~m} / \mathrm{s} 2$ ) per digital level. 
Table 7: Acquisition characteristics

\begin{tabular}{|c|c|c|}
\hline \multirow{3}{*}{$\frac{0}{\frac{0}{2}}$} & ADC Resolution (bits) & $10\left(2^{10}\right.$ levels $)$ \\
\hline & Range (Volts) & 3.3 \\
\hline & Resolution (V/level) & $3.3 / 2^{10}=0.0032$ \\
\hline \multirow{3}{*}{ 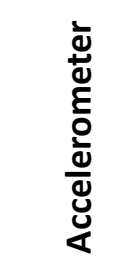 } & Acquisition rate $(\mathrm{Hz})$ & 100 \\
\hline & Sensitivity (V/g) & 0.8 \\
\hline & Resolution (g/level) & 0.004 \\
\hline
\end{tabular}

The equipment is prepared to work standalone. The battery was able to give power for around 3 hours. It was easily replaced each 2 hours to avoid unstable recordings.

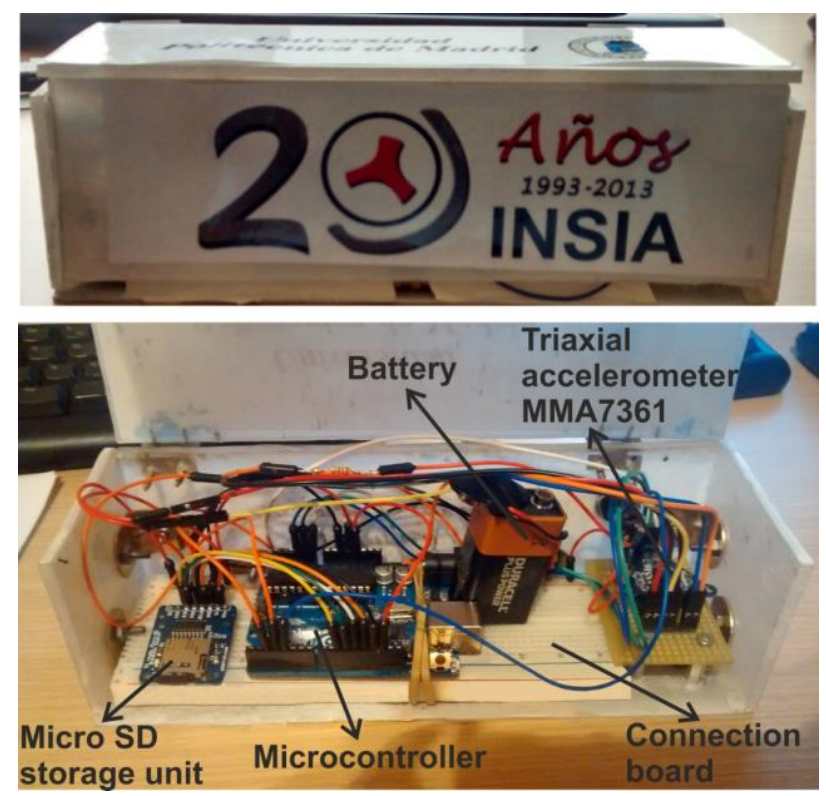

Figure 44 Vertical accelerations acquisition system.

Recordings were obtained from 5 different LPTV models of the EMT during normal operation over 5 different lines. In all, more than 8 hours of bus service were recorded. The acquisition frequency used was $100 \mathrm{~Hz}$.

\subsubsection{EVALUATION OF THE ACCELEROMETER MEASURING QUALITY}

In order to ensure that the resolution and precision of the accelerometer recordings are sufficient for the propose, several evaluations of the measuring quality were performed.

First, with the aim of assessing the stability and precision of the acquisition equipment, recording during steady state (i.e. no accelerations happening) were taken over 45 minutes, which is approximately the duration of a bus route. The resulting raw data of vertical acceleration time series and its histogram distribution are show in Figure 45. 

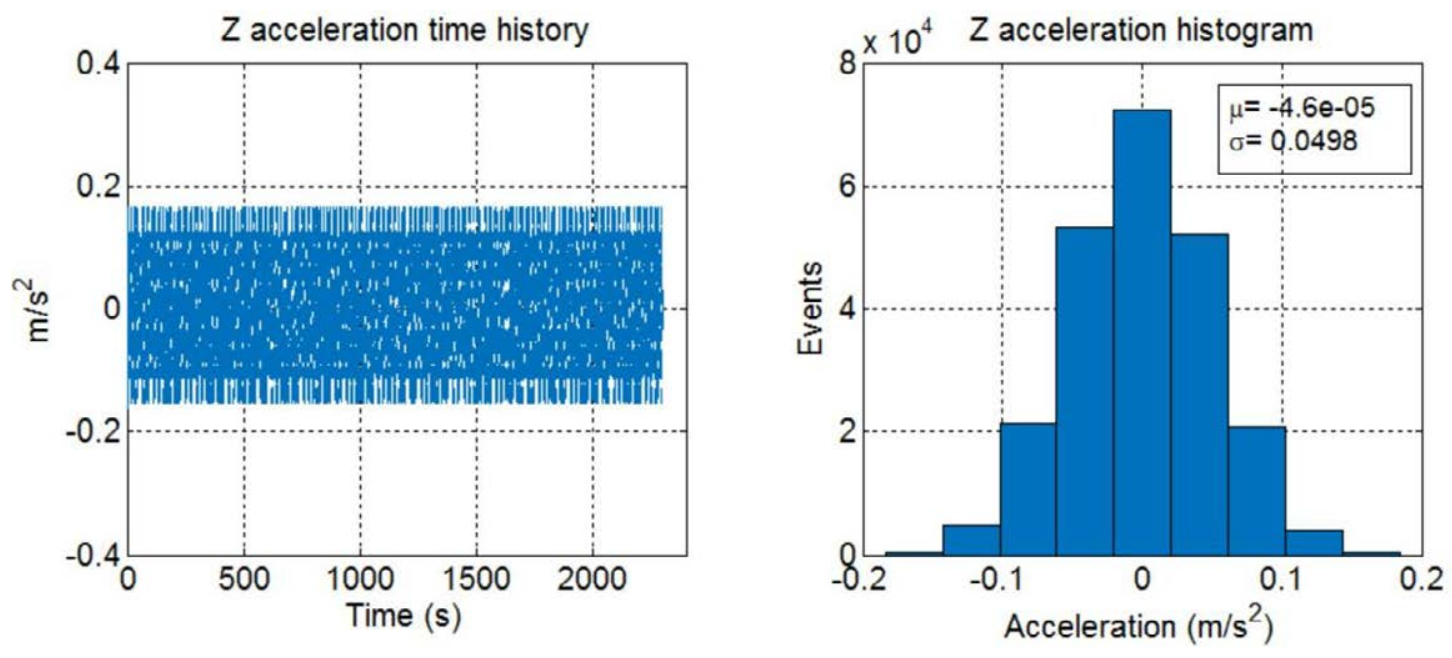

Figure 45 Vertical accelerations precision and stability evaluation.

From the observation of the left hand image of Figure 45, any drift on the acquisition equipment can be discarded, since the recordings remain stable over all measured time. The insignificant mean acceleration observed $\left(\mu=-4.6 \mathrm{e}^{-5}\right)$ in the histogram graph of the left hand image ratifies this behavior.

On the other hand, signal precision is a characteristic that depends not only on the accelerometer properties but also on the overall acquisition system, since quality of the wiring and connections can also aid further noise to the signal records. For a quantitative assessment of the noise influence on the acceleration measurements, it is necessary to compare the data dispersion obtained in stationary conditions with the recordings during real operation of the LPTV. To this end, an analog histogram to the one of Figure 45 have been obtained, but in this case from all recorded data (Figure 46).
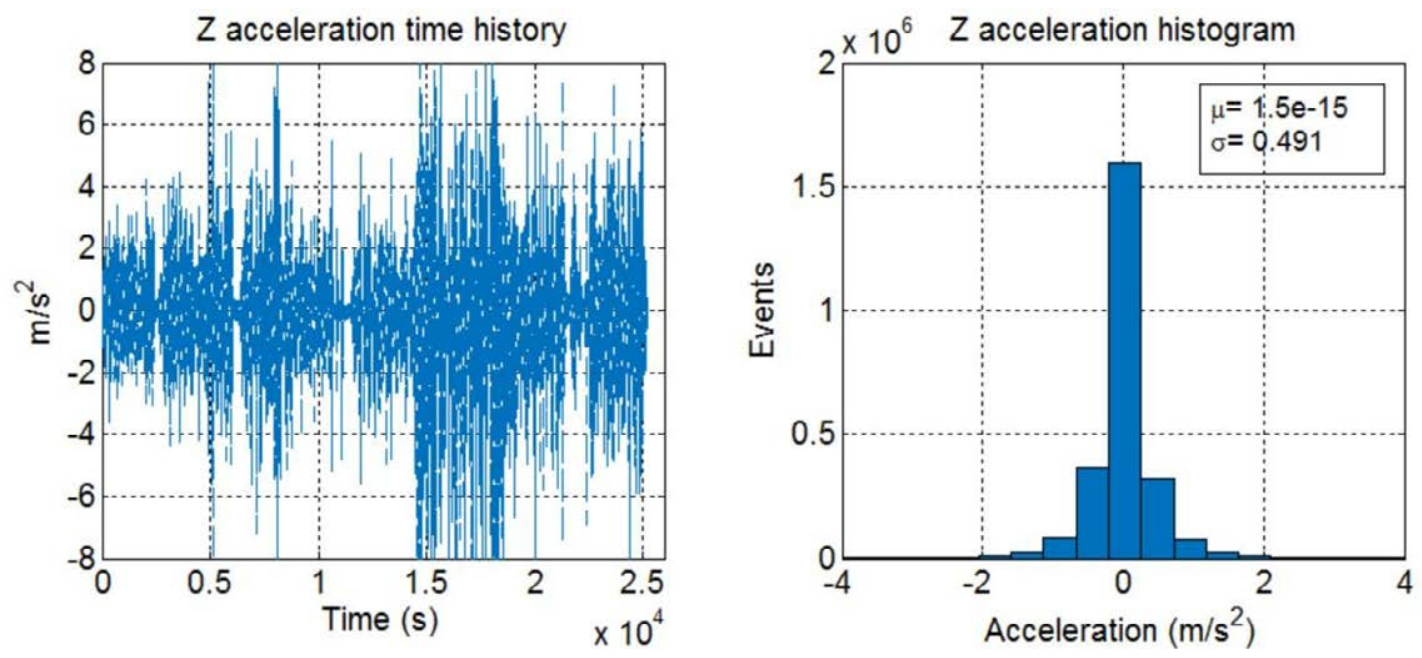

Figure 46 Vertical accelerations distribution during operation.

It is observed that noise is approximately one order of magnitude lower than the real test data dispersion. It should be noted that these results correspond to raw data, and thus further improvement of the precision will be achieved on the signal after the signal filtering presented in the next section. 
Finally, it is worth to notice that the probability distribution of the $\mathrm{Z}$ accelerations during LPTV normal operation is zero centered (since the overall mean is $\mu=1.5 \mathrm{e}^{-15}$ ) and has an important density at very low acceleration data. The same behavior was observed of lateral and longitudinal accelerations (J. Pérez et al. 2014).

\subsubsection{DATA FILTERING}

The procedure of filtering raw input data has the aim of reducing the noise and thus improving the precision by producing a clearer signal. Nevertheless, it is important to apply a correct filtering method to avoid missing information during the process.

For this purpose, a lowpass Butterworth filter was used. This type of filter reduces and rolls off towards zero data from the signal that occurs at higher frequencies than the cut-off established frequency $\left(w_{c}\right)$ according to the following frequency response $(H)$ equation:

$$
H(\omega)=\frac{1}{\sqrt{1+\left(\omega / \omega_{c}\right)^{2 n}}}
$$

Where $w$ is the frequency and $n$ is the Butterworth filter order. As can be deduced from the equation, the higher the order, the more pronounced will be the response decrease from the cutoff frequency.

In order to ensure no loss of information, the cutoff frequency should established in such way that only noise is removed from the signal. To determine this value, the frequency spectra of records obtained from stationary and real test conditions were compared (Figure 47). As it can be distinguished, the difference between actual acceleration amplitudes and noise becomes negligible from $15 \mathrm{~Hz}$ approximately. A conservative cutoff frequency of $20 \mathrm{~Hz}$ will be used to filter the acceleration data.
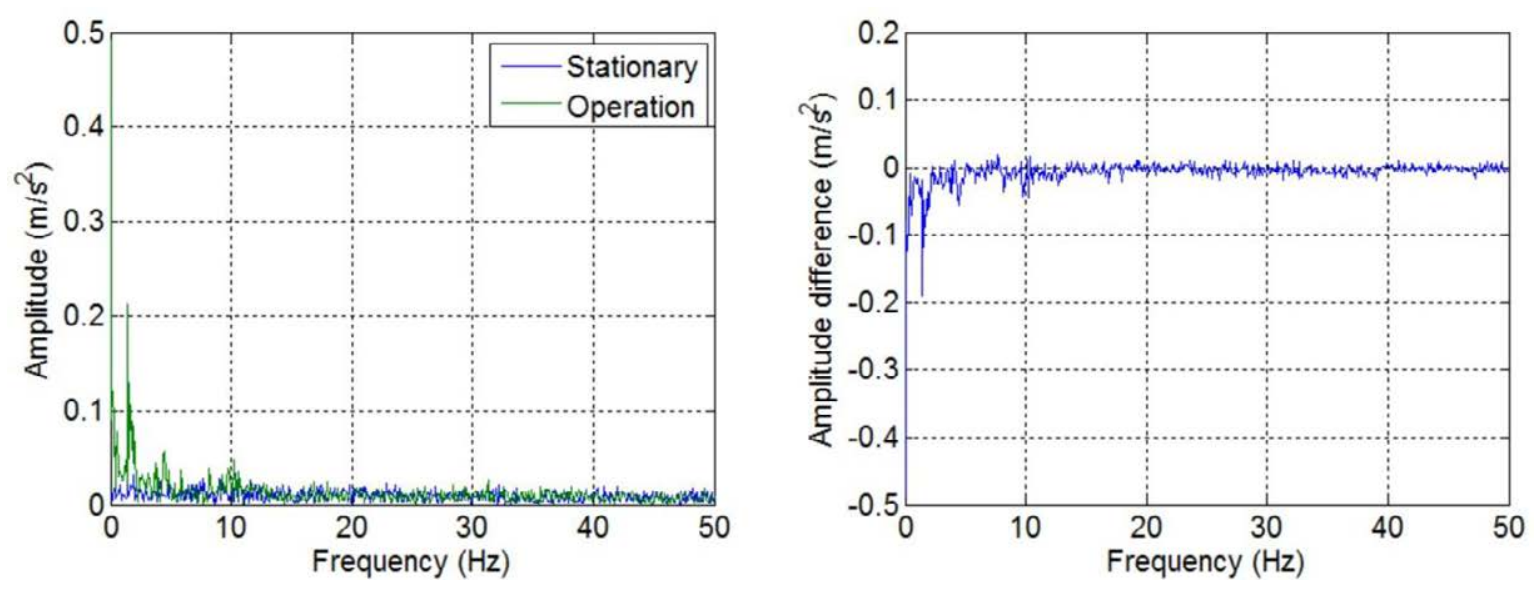

Figure 47 Comparison of accelerations in frequency domain.

Additional information can be extracted from the observation of Figure 47. First, most significant accelerations occur at low frequencies. Second, there is an amplitude raise at $2.5 \mathrm{~Hz}$ 
approximately, that most likely corresponds to the natural mode of vibration of the LPTVs sprung mass. Finally, some minor acceleration amplitudes are yet observed at frequencies up to $15 \mathrm{~Hz}$, evidencing the impossibility of a common GPS system to characterize this type of accelerations.

The frequency response and the resulting signal in frequency domain after the application of the 6th order Butterworth filter with a cutoff frequency of $20 \mathrm{~Hz}$ are shown in Figure 48 . This filter applies a maximally flat respond in the passband (no ripples), and considers the cutoff frequency at which the magnitude response of the filter is $1 / 2^{0.5}$.
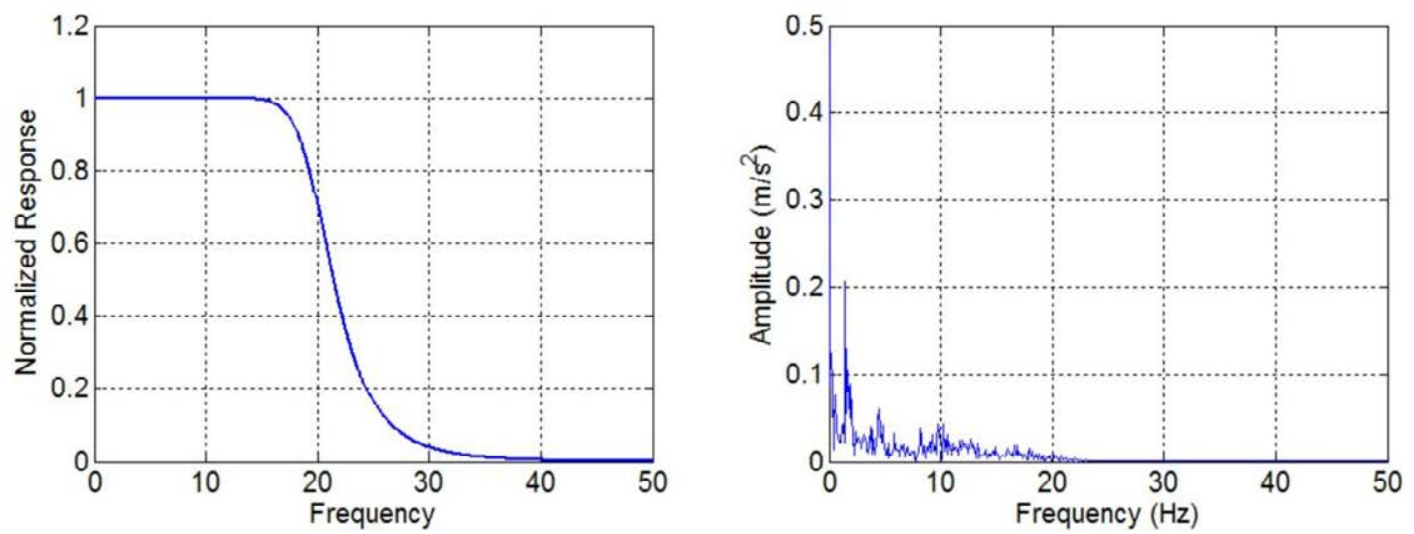

Figure 48 Butterworth filter application.

As expected, most significant amplitudes are kept unchanged, whereas noisy amplitudes that occur at higher frequencies are removed from the signal. The resulting acceleration data will be used as input for the cycle counting presented in the following section.

\subsection{LOAD CYCLES: IMPLEMENTATION OF RAINFLOW CYCLE COUNTING METHOD OVER THE ACCELERATION TIME SERIES}

The extraction of fatigue load cycles from time series is a necessary operation that enables posterior evaluation of fatigue damage on the structure. Additionally, these time series are sometimes sufficiently complex that fatigue cycles cannot be easily distinguished, as is the case of the LPTV accelerations recorded. In these cases, specific techniques of cycle counting must be applied. In the present thesis, the Rainflow counting method introduced in Section 3 will be employed.

The obtained acceleration cycles for lateral and longitudinal accelerations over 600 hours of operation are shown in Figure 49 and Figure 50 respectively. Due to the availability of the information about instant position and velocity, events that corresponded to bus stops have been removed from the time series to speed up the counting process. First and last stop instants have been kept to ensure no cycle loss during the beginning and end of the stop. Also, the graphs limits have been set to ensure enveloping maximum amplitude cycles.

It can be observed that for both lateral and longitudinal accelerations, high alternate acceleration cycles happen at low mean accelerations values and vice versa. High alternate and mean acceleration cycles would mean, in the case of lateral acceleration, performing a notable 
turning maneuver when already in a significant turn. For the longitudinal ones, it would refer to a sharp break or acceleration maneuver when already breaking of accelerating significantly. These situations are not very logic to happen during normal circulation and could lead to vehicle instability or even accidents. It is also seen that for low lateral acceleration cycles, the higher densities are not centered at zero mean. This is most likely due to the fact that during a complete turning maneuver (zero centered cycle), there are multiple small direction corrections from the driver that suppose nonzero accelerations cycles.

\section{Lateral acceleration cycles}

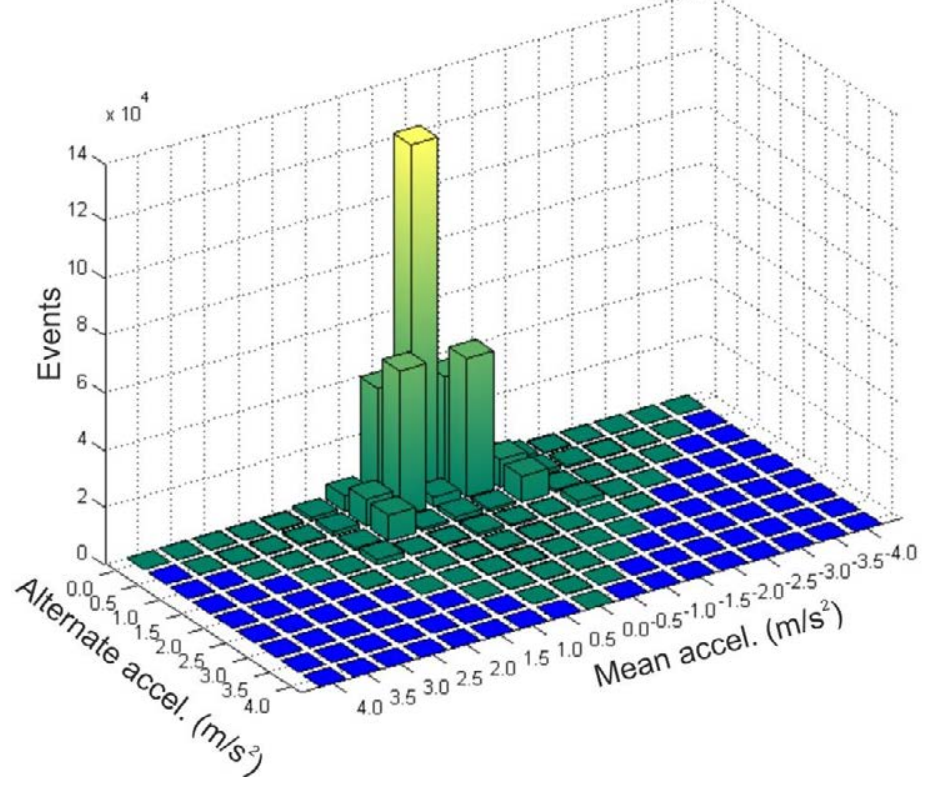

Figure 49 Rainflow cycle counting results for lateral accelerations.

\section{Longitudinal acceleration cycles}

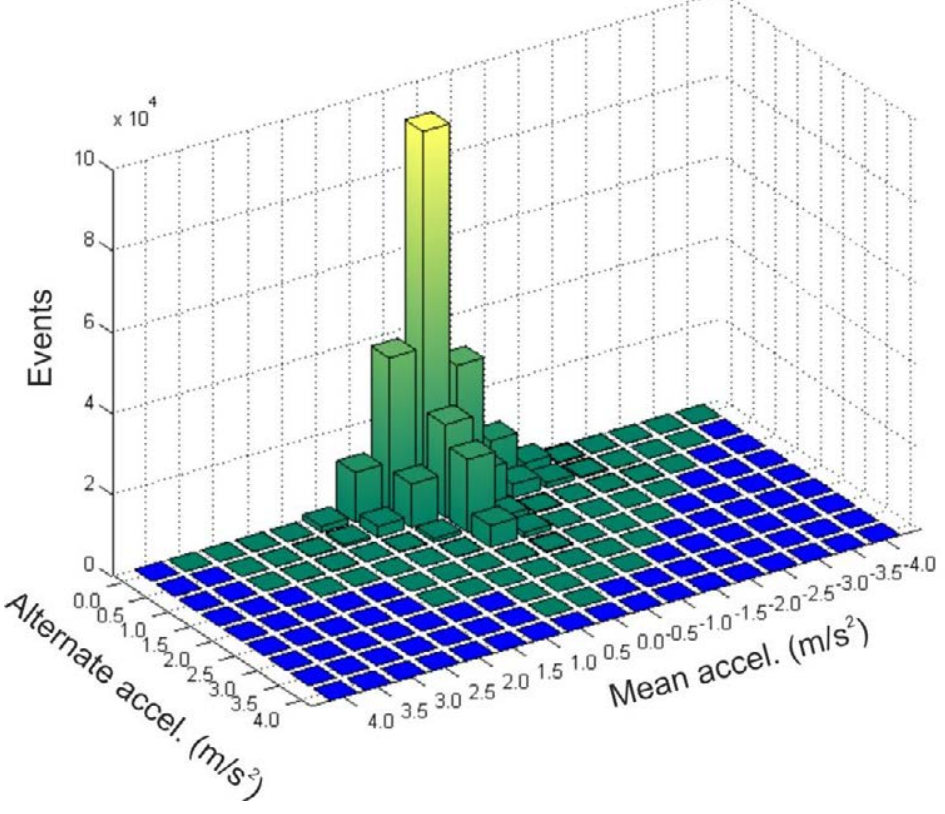

Figure $\mathbf{5 0}$ Rainflow cycle counting results for longitudinal accelerations.

The analog results obtained for vertical accelerations are plotted in Figure 51. Tendency of the cycles distribution is not as clear is in the lateral and longitudinal cases, since these 
accelerations do not come from vehicles maneuvers controlled by the driver, but from road imperfections. Additionally, occasional high alternate acceleration cycles have been located even thought the global standard deviation of vertical accelerations is not higher than the lateral or longitudinal ones. Finally, a high number of events very close to zero mean and alternate values can be noted, most likely caused by cycles accounted from signal noise during bus stops.

\section{Vertical acceleration cycles}

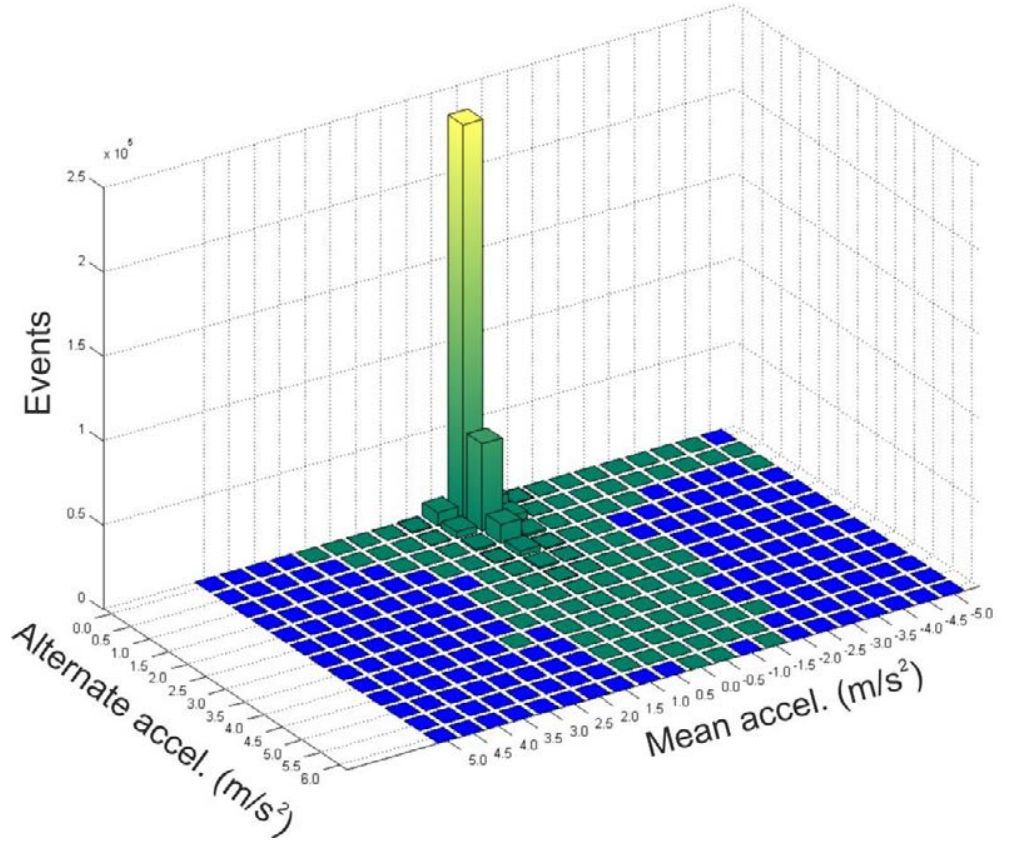

Figure 51 Rainflow cycle counting results for vertical accelerations.

It should be noted that the number of events of the vertical accelerations is higher than for the lateral and longitudinal ones in relation to the recorded time. This is due to the higher frequency at which this type of accelerations occur as shown in Figure 47, and to the acquisition rate for the high central density .

The acceleration cycles thus obtained, extrapolated to the desired lifespan of the vehicle will be used as the load input in the simplified model of the complete bus structure for the fatigue damage assessment. 


\section{STRUCTURAL AND FATIGUE CHARACTERISTICS OF WELDED RECTANGULAR TUBE BEAMS}

The present section covers all the experimental techniques carried out with the aim of characterizing the structural behavior of the rectangular beam welded junctions.

As mentioned in the introductory section, the $\varepsilon-\mathrm{N}$ approach will be employed to assess fatigue damage. This approach is most appropriate when notches are present on the fatigued component. A complete $\varepsilon-\mathrm{N}$ approach requires, on the on hand, knowing the flow curve of the material where highest strains are present (i.e. where failure is expected to occur), since this information will be used to separate elastic and plastic strain components from the total strain suffered by the junction during fatigue cycles. Also, the peak total strain needs to be known, since this will be the input strain value on the $\varepsilon-\mathrm{N}$ curve. Finally, a sufficient number of fatigue tests on these welded junctions must be performed to construct a representative $\varepsilon-\mathrm{N}$ curve.

In the following sections, these characterization experiments and the results obtained are presented in further detail.

\subsection{STRESS-STRAIN CURVE OF WELDED STRUCTURAL STEEL}

When a structural steel is subjected to a welding process, its microstructure and in turn its mechanical properties change in the vicinity of the weld bead (Z.L. Zhang et al., 2002). In the one hand, the filler material used during the welding process has usually different characteristics from those of the base material. Additionally, next to this filler material arises a zone where the material, although not melted, change its properties due to the heat input. This so-called heat affected zone (HAZ) presents a gradient on the material properties from the filler to the base material.

The microstructure and size of the HAZ depends on the chemical composition and the welding conditions (R. Kacar \& K. Kökemli, 2005). In general terms, grain size tends to grow in this zone, decreasing the material strength. In mig-mag arc welded rectangular tube beam structural steels typical of LPTV structures, the length of the HAZ is roughly between 3 and $5 \mathrm{~mm}$ (H. Malón, 2010; Z. Boumerzoug et al. 2010). Consequently, peak strains during fatigue loadings, that concentrate along the weld toe, will lie within the HAZ.

Despite that the influence of the HAZ on fatigue crack grown is believed to be limited (S.J. Maddox, 1992), it is important for the $\varepsilon-\mathrm{N}$ curve determination since fatigue failure occurs at this zone. Consequently, the characterization of the stress-strain curve at this zone becomes necessary to obtain elastic and plastic components of the total strain during fatigue loads.

\subsubsection{SPECIMEN CHARACTERISTICS AND TEST SETUP}

The dimensions of the welded specimens employed or the flow curve characterizations is shown in Figure 52 (a). They were produced by water cutting from a welded plate. A milling operation was later performed to remove the protruding material to avoid strain concentrations 
and facilitate measuring process (Figure 52 (b)). The weld bead was strategically placed in such way the one end lies at the middle of the gauge zone of the specimen. This, coupled with the small gauge zone, increases the probabilities of breakage in the HAZ zone. The base material used was S275JR, very common among LPTV structures, and the one used for the fatigue tests. Its mechanical properties according to the UNE-EN_10025 Standard are gathered in Table 8. The filling material was a $\varnothing 0.8 \mathrm{~mm}$ solid welding wire roll brand 'Zahurin', recommended for structural steels and following the standard UNE-EN440.

Table 8: Mechanical properties of S275JR according to UNE-EN_10025 Standard

\begin{tabular}{|c|c|}
\hline Property & Value \\
\hline Yield stress (MPa) & 275 \\
\hline Ultimate strength (MPa) & $410-560$ \\
\hline Strain to fracture (\%) & $15-19$ \\
\hline
\end{tabular}

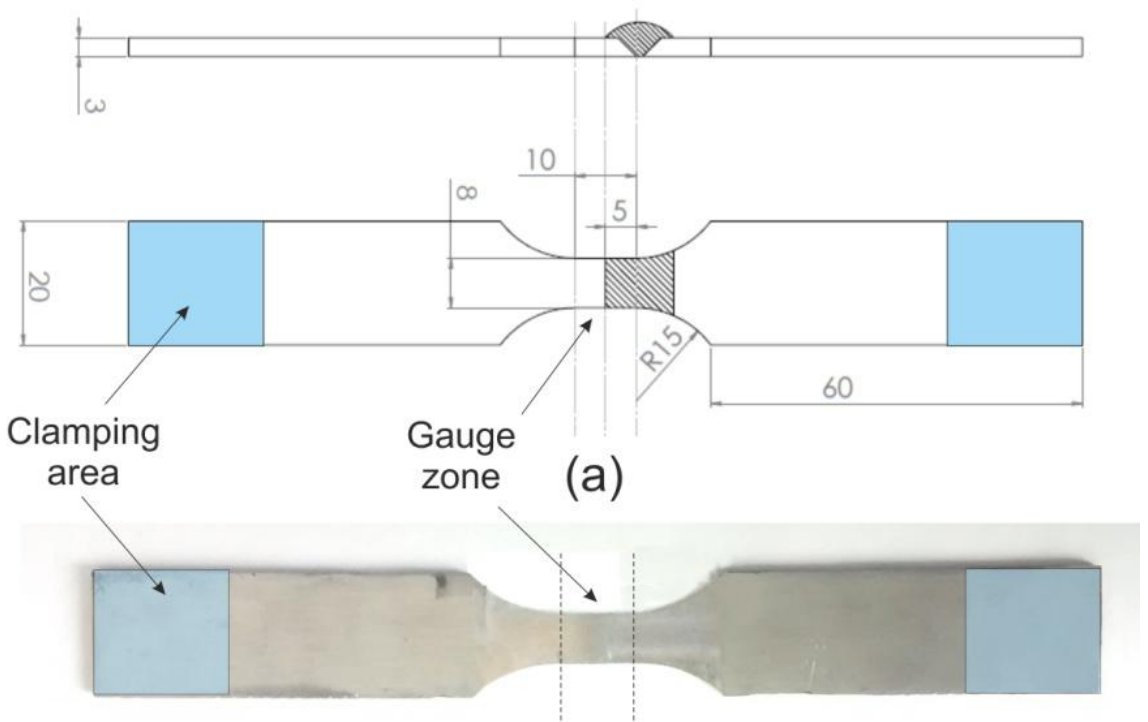

(b)

Figure 52 Tensile test specimen dimensions

Three specimens were tested using an $100 \mathrm{kN}$ Instron ${ }^{\circledR}$ Model1362 tensile machine. To extract instant strain values, a DIC equipment was used, which was triggered with the force analog signal from the tensile machine to ensure synchronized recordings for stress and strain determination. Using the DIC technique allows extracting the strain evolution during the tensile test at any location of the focused area. Furthermore this technique permits accurate estimations of the cross sectional area reduction during the test, which enables the determination of the true stress evolution. The test setup is shown in the following Figure 53. 


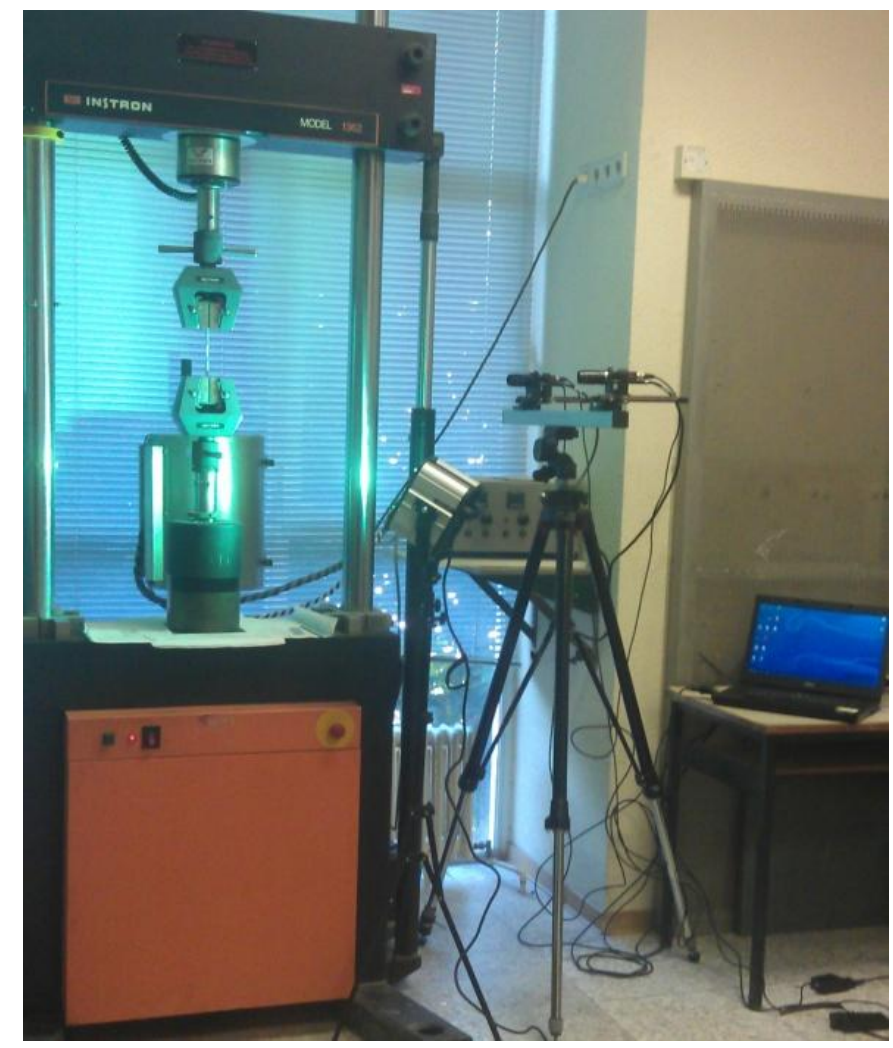

Figure 53 Tensile test setup

For the image correlation algorithm to work properly, a random contrast pattern over the measured surface must be created. This pattern is generated by first applying a spray of white matt paint followed by a black random speckle also applied with spray paint. Figure 54 shows an image taken by one of the DIC cameras of a speckled specimen in unloaded conditions.

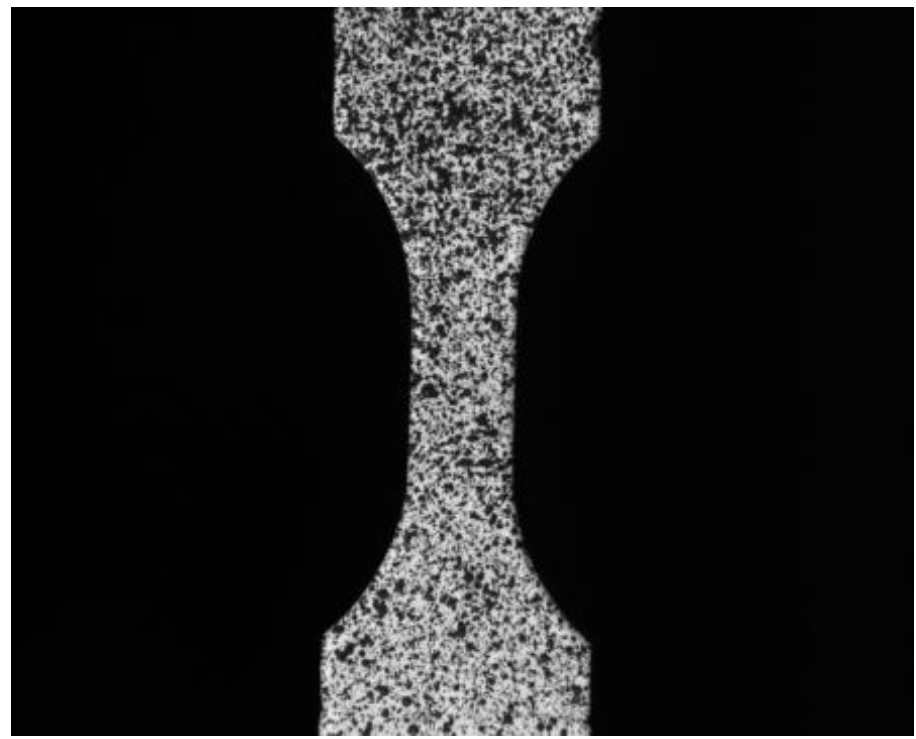

Figure 54 Speckled specimen

All images were processed using the commercial DIC software VIC3D. The processing settings utilized for image correlation and strain calculation are summarized in Table 9. 
Table 9: DIC processing settings

\begin{tabular}{|c|c|}
\hline Subset size & 33 pixels \\
\hline Step Size & 7 pixels \\
\hline Strain Window & 21 data points \\
\hline Strain tensor & Lagrange \\
\hline Pixel size & $0.025 \mathrm{~mm}$ \\
\hline
\end{tabular}

By way of example, Figure 55 shows the strain distribution over one of the specimens at three different stages of the test. It is appreciated how maximum strains concentrate at the center of the gauges length (i.e. where the HAZ is located). Significant necking of this zone is observed in the right image, which was taken just before specimen breakage.

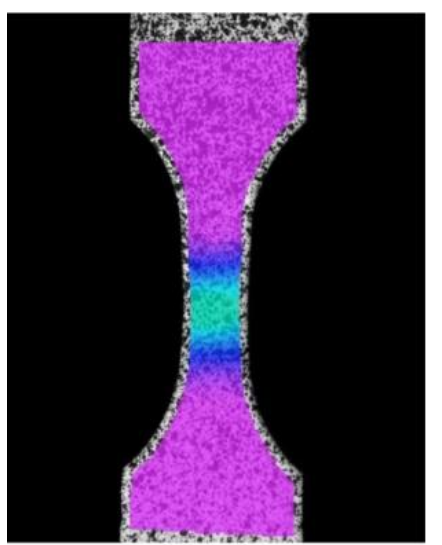

0

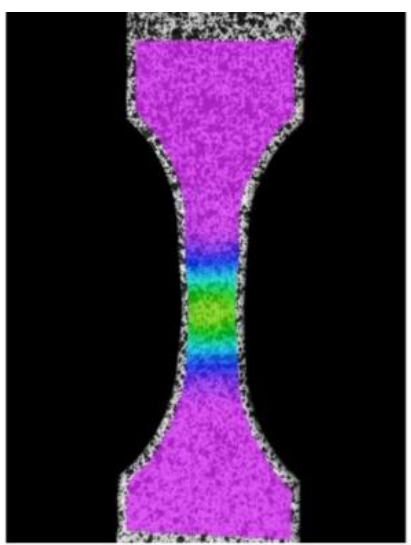

22.5

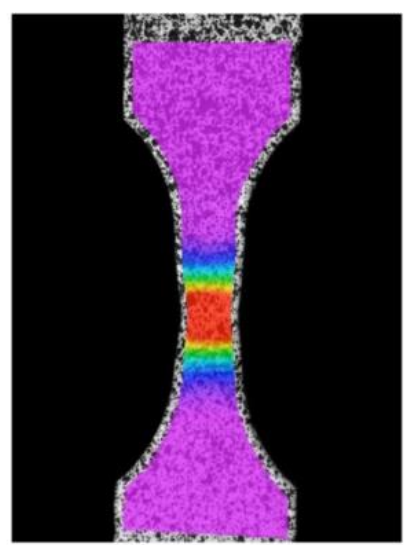

33.75
45

$\varepsilon_{y y}(\%)$ - Lagrange

Figure 55 DIC processed images

\subsubsection{TEST RESULTS: HAZ MATERIAL PROPERTIES}

The evolution of the strain was evaluated at a data point in the middle of the gauge zone. The following Figure 56 shows the monotonic tensile engineering and true stress-strain curves obtained. DIC equipment outputs strain measures according to Lagrarian tensor. These values were later used to calculate true and engineering strains by means of the stretch gradient described in Section 3.3.1. On the other hand, engineering stress was determined from the force acquisitions and the initial area of the gauge zone of the specimens. True stress was obtained from its relation to engineering stress and strain presented in (1.48). Strain values belonging to the necking process have been removed from the calculation since they can skew the determination of the strain hardening parameters $K$ and $n$. 


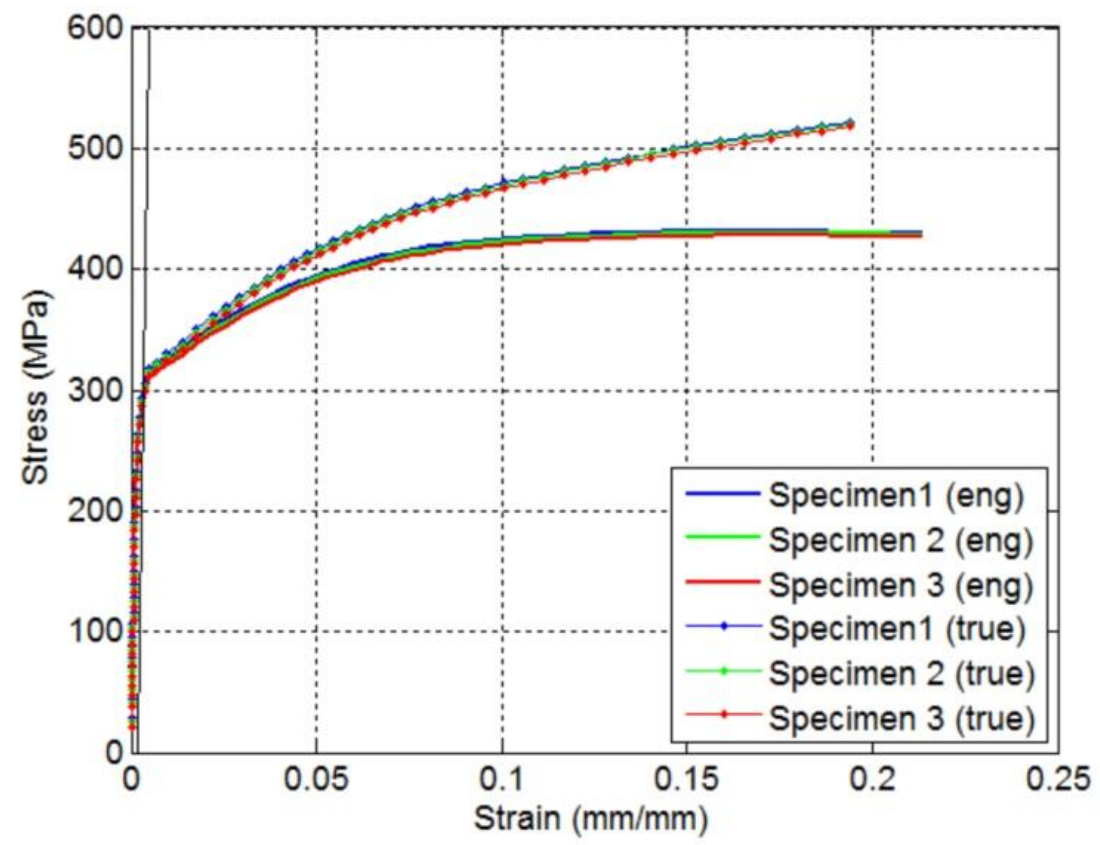

Figure 56 Monotonic engineering and true stress-strain relation

From these results, elastic modulus was calculated as the slope of the linear fit of stress strain data, always for stress values under the proportionality limit, according to the ASTM E11104 Standard. Young modulus enables separating elastic from plastic components of strain, and thus determining the strain hardening properties of the material by first order power fitting according to eq. (1.52) (Figure 57). The region between the yield and ultimate strength points was considered for the strain hardening parameters calculation according to the recommendations of the Standard ASTM E646-16.

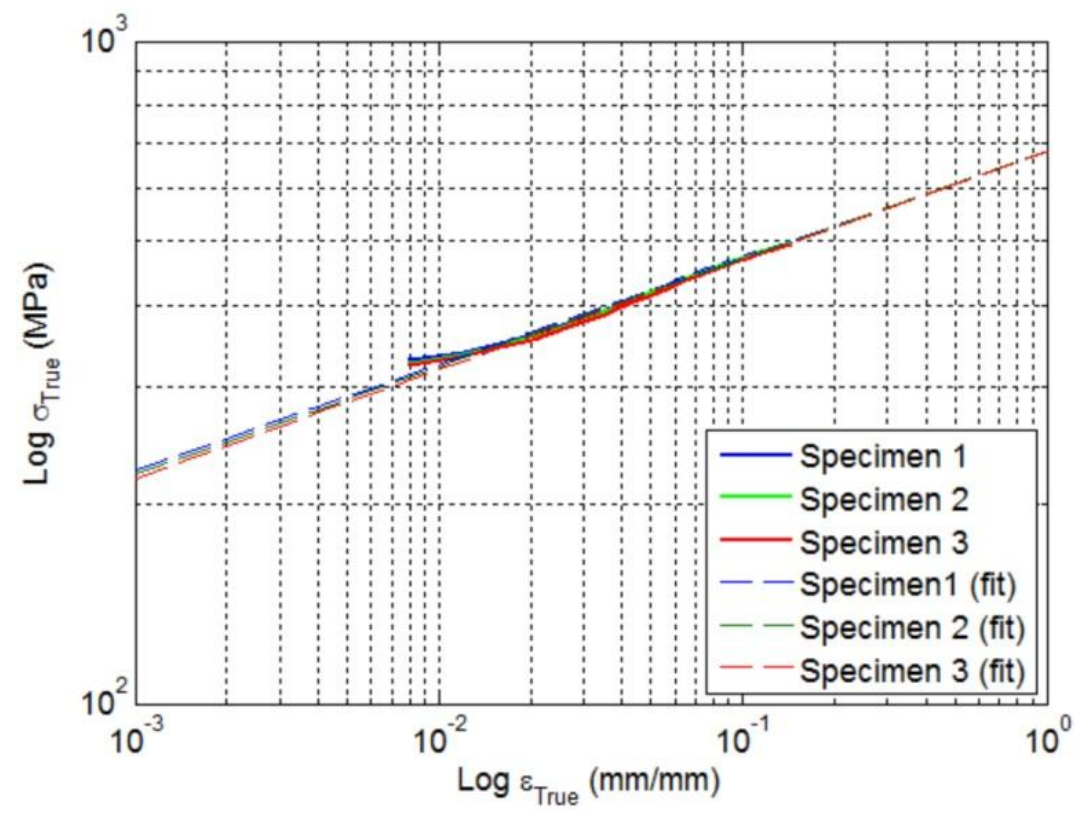

Figure 57 Strain hardening fit

Yield stress was determined according to the $0.2 \%$ offset method proposed by the ASTM A370-10 Standard, as the intersection between the flow curve, with the slope of Young Modulus that starts with a strain offset of $0.2 \%$. Practically, it refers to a yield stress associated with a 
$0.2 \%$ plastic strain. Finally, the ultimate stress is easily obtained as the maximum stress achieved during the tensile tests. The following Table 10 gathers all the characteristic properties of the $\sigma-\varepsilon$ relation of the material.

Table 10: Stress-strain properties

\begin{tabular}{|c|c|c|c|c|}
\hline Property & Test 1 & Test 1 & Test 1 & Mean \\
\hline Young Modulus (GPa) & 202.6 & 202.6 & 202.7 & 202.6 \\
\hline Yield stress (MPa) & 301.4 & 298.1 & 294.5 & 298 \\
\hline Ultimate strength (MPa) & 431.2 & 430.1 & 427.9 & 429.7 \\
\hline $\begin{array}{c}\text { Strain hardening } \\
\text { exponent (n) }\end{array}$ & 0.160 & 0.163 & 0.164 & 0.162 \\
\hline $\begin{array}{c}\text { Strength coefficient (K) } \\
\text { S }\end{array}$ & 680 & 681.9 & 680.6 & 680.1 \\
\hline
\end{tabular}

\subsection{STRAIN CONCENTRATION BEHAVIOR OF RECTANGULAR TUBE BEAM WELDED JUNCTIONS}

The protruding weld bead that remains from the welding process supposes a sharp geometric change that acts as a local strain raising (i.e. strain concentration). The location and magnitude of this strain raising strongly depends on the local shape characteristics of the weld bead. During the welding process, the exact geometry of the weld bead and the location of the bead waves are usually uncontrolled. Consequently, the magnitude and location of the maximum strain concentration is unknown in advance, compelling to experimental methods to characterize this behavior.

The DIC technique was employed in this thesis to evaluate the strain concentration on rectangular beam welded junctions typical on LPTV structures. The possibility of obtaining strain fields over a whole area of interest ensures capturing the maximum peak strain value regardless its location. The following subsections describe the welded junctions characteristics, test setup, and the results obtained for this characterization. The junctions characteristics and most of the test setup configurations will also apply to the fatigue tests of section 5.3.

\subsubsection{WELDED JUNCTION CHARACTERISTICS}

The great majority of the bus body structures are fabricated by means of rectangular section structural steel beams, joined by means of a welding process. This technique is the most extended solution for bus body manufacturing, since it supposes a flexible manner of producing a structure that fits the chassis of the vehicle with the needed resistance and stiffness characteristics. 
Most of the junctions that constitute a LPTV structure are profiles welded at 90 degrees, forming the so-called T-junctions. Regarding the dimensions of these profiles, it is a very common choice to use standardized sizes, since they suppose a less costly and greater availability option inasmuch as they are used for other many structural applications. Commonly used profile dimensions range from sections sides of 20 to $80 \mathrm{~mm}$, and 2 to $4 \mathrm{~mm}$ of thickness. By way of example, Figure 58 shows a CAD model of a LPTV structure.

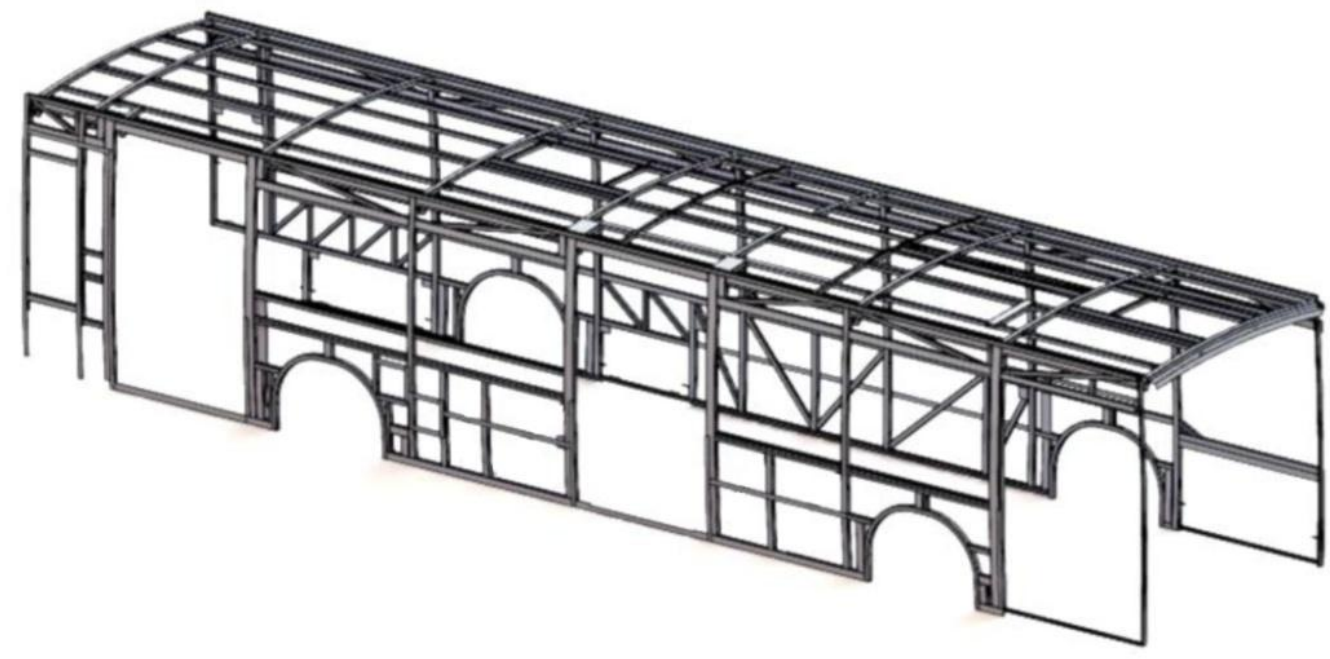

Figure $58 \mathrm{Cad}$ model of bus body structure

With respect to the material, different steels are employed for these structures. For example, higher strength structural steels are usually employed in parts of the structure involved in the energy absorption during rollover. On the contrary, more ductile materials are preferred for zones with little structural importance in order to save unnecessary costs.

In any case, the great majority of the steels used for LPTV structures are structural steels, which present very similar elastic properties. Also, during the welding process the properties of these materials are modified, increasing the grain size and thus lowering the material strength. Furthermore, it should be pointed that, in contrast with unwelded materials, fatigue failure in welded joints does not vary significantly with the material tensile strength (S.J. Maddox, 1992). Consequently, the material chosen for the structural analysis will have none or little impact on the strain concentration neither in the fatigue behavior of the welded joint.

In this scenario, two different welded junctions representing two possible configurations of T-welded profiles were chosen for the structural characterization. The dimensions of this structural components is shown in Figure 59. In both cases the profile section dimensions are $60 \times 40 \times 3 \mathrm{~mm}$. Finally, the material used for the tube profiles and for the weld filling was the same as in the monotonic material characterization of previous section. 


\section{Type A}

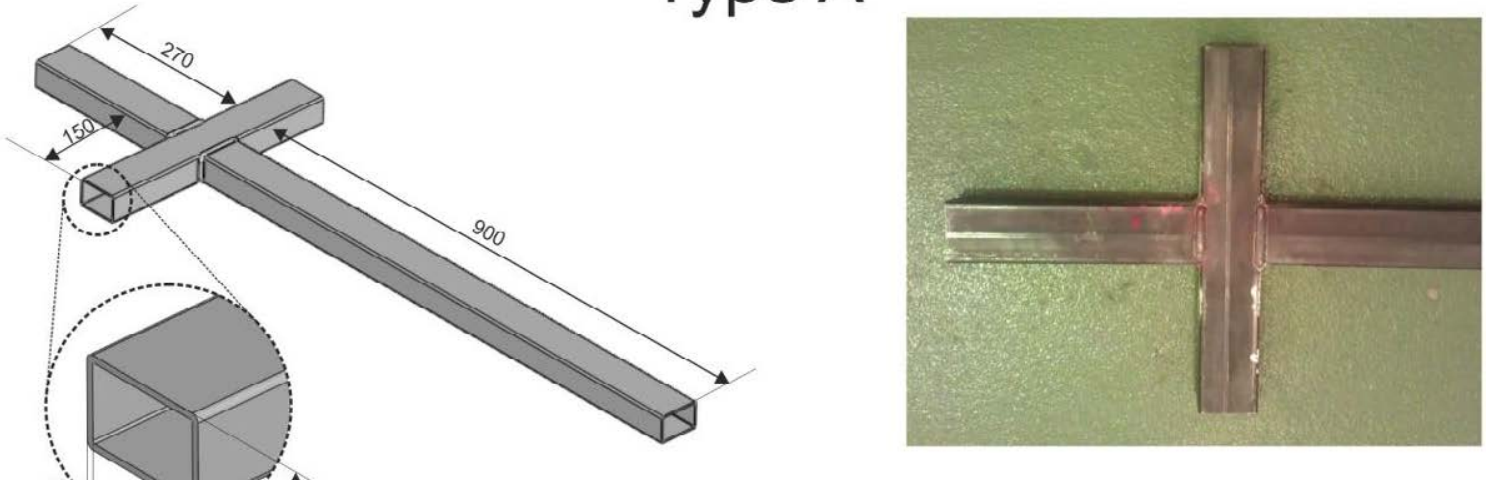

\section{Type B}
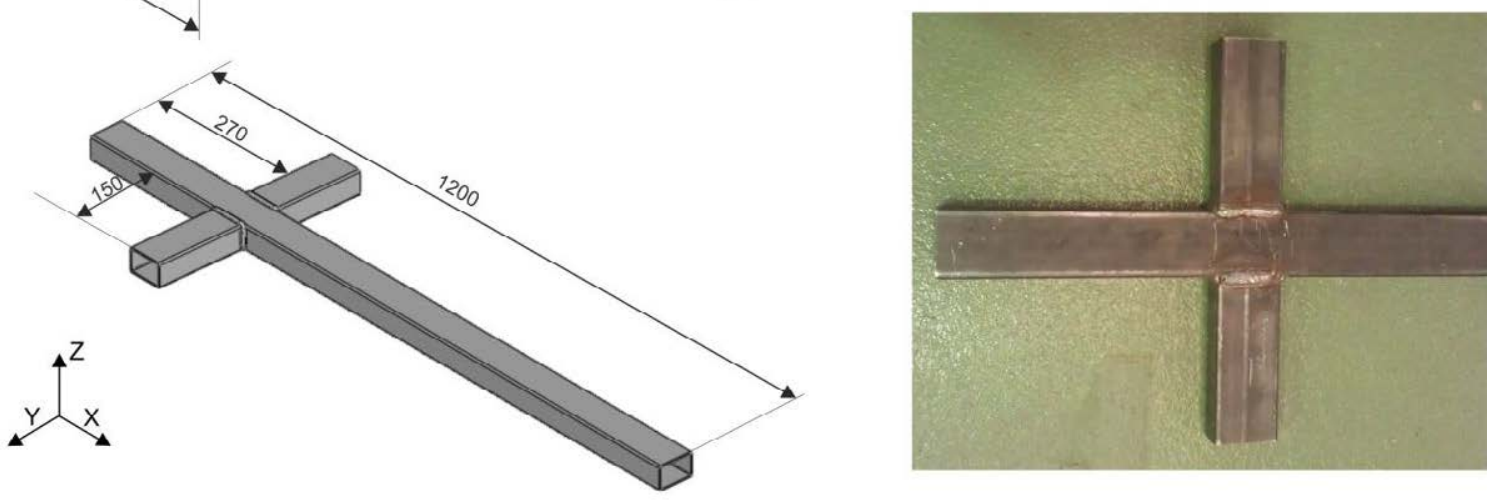

Figure 59 Welded junctions studied

\subsubsection{TEST SETUP}

For both welded junction types, the shorter profile braches were used to constrain the displacements at controller distances from the junction as shown in Figure 60.

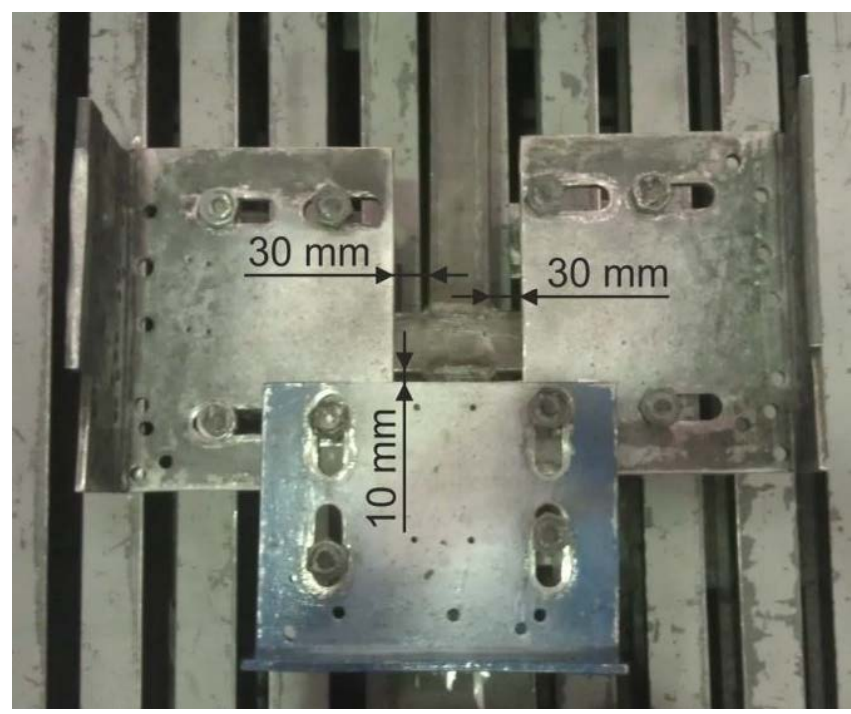

Figure 60 Displacement constrain support

The longer profile branch of the junction was connected to a hydraulic actuator Instron Schenk 1-PKT-2301. This actuator can supply a maximum force of $22.5 \mathrm{kN}$, and has a cylinder 
displacement range of $250 \mathrm{~mm}$. In order to record force and displacement data, the equipment is installed with a high precision load cell PM-K-25 (Table 11) designed for components testing and an differential inductor displacement transducer WLC250 respectively (Table 12).

Table 11: PM-K-25 load cell technical data

\begin{tabular}{|c|c|}
\hline Nominal Force & $25 \mathrm{kN}$ \\
\hline Nominal output signal & $1.6 \mathrm{mV} / \mathrm{V}$ \\
\hline Supply voltage & $\leq 10 \mathrm{~V}$ \\
\hline Relative nonlinearity & $0.02 \%$ \\
\hline Cross force effect & $0.2 \%$ \\
\hline Operating temperature range & $10 \circ \mathrm{C} . . . . .60 \circ \mathrm{C}$ \\
\hline
\end{tabular}

Table 12: WLC250 displacement transducer technical data

\begin{tabular}{|c|c|}
\hline Nominal Range & $\pm 125 \mathrm{~mm}$ \\
\hline Nominal output signal & $80 \mathrm{mV} / \mathrm{V}$ \\
\hline Supply voltage & $\leq 5 \mathrm{~V}$ \\
\hline Relative nonlinearity & $0.4 \%$ \\
\hline Operating temperature range & $-55 \circ \mathrm{C} . . . .100$ 으 \\
\hline
\end{tabular}

A total of 10 different welded junctions were measured for this study, each subjected to controlled loads applied in ' $Z$ ' and ' $Y$ ' directions according to the coordinate system of Figure 59. The point of load application was at the controlled distance of $840 \mathrm{~mm}$ to the closest face of the junction to the hydraulic cylinder. As it is observed in Figure 61 , ' $Z$ ' direction loads were directly applied by the actuator, where as to apply the load in ' $Y$ ' direction it was necessary to add a pulley in order to change the load direction by 90 . It should be noted that the test setup needed to be changed for positive and negative senses of ' $Y$ ' direction loads.
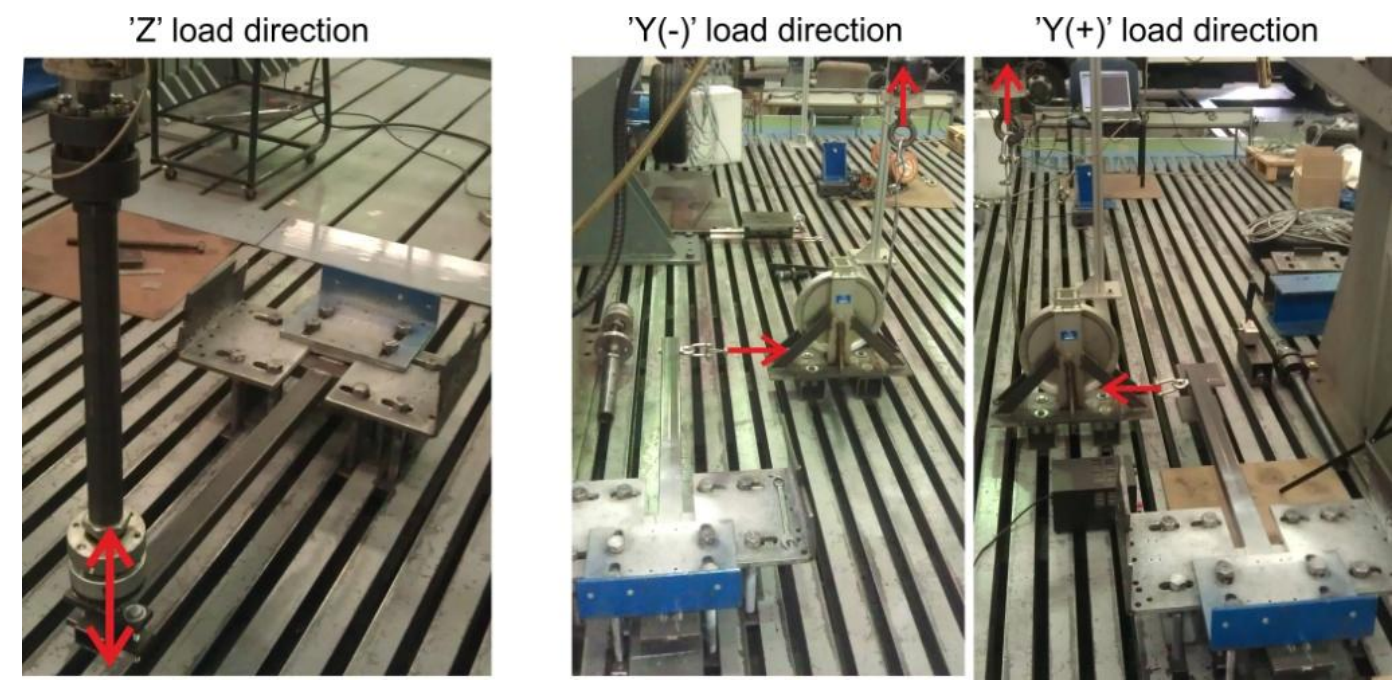

Figure 61 Welded junctions test installation 
The load was applied in form of controlled displacements ranged from $-12.5 \mathrm{~mm}$ to $12.5 \mathrm{~mm}$ with incremental load steps of $2.5 \mathrm{~mm}$. Nevertheless, outcomes from displacements of $\pm 2.5 \mathrm{~mm}$ were later discarded from the study since they produced such small strain fields that are irrelevant from the fatigue point of view, and were difficult to process with DIC due to the high influence of noise at these strain values.

The displacement in ' $\mathrm{Z}$ ' direction was directly controlled by the acquisition system integrated in the actuator. On the other hand, to control the displacement of the junction in ' $Y$ ' direction, the integrated system cannot be used due to the elongation of the cable used to transform the load direction. For this reason, it was installed an extra draw-wire displacement sensor ASM WS10 (Table 13) aligned with the load application point to register the true displacement of the junction.

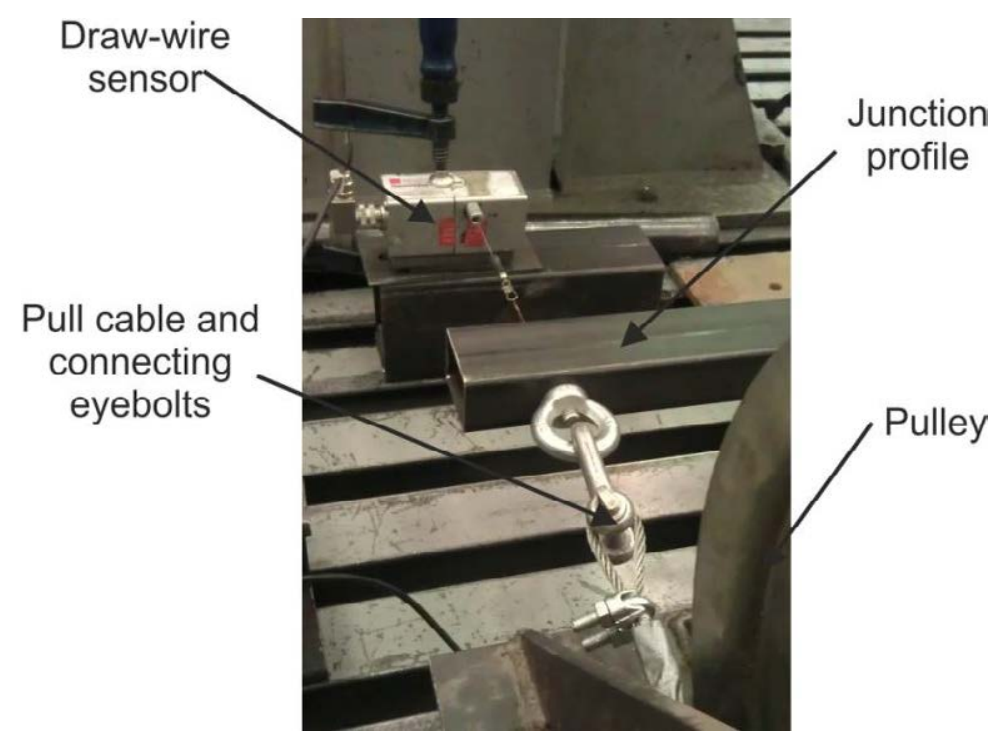

Figure 62 Draw wire displacement sensor

Table 13: ASM WS10 wire displacement sensor technical data

\begin{tabular}{|c|c|}
\hline Nominal Range & $\pm 1000 \mathrm{~mm}$ \\
\hline Output Voltage & $0-10 \mathrm{~V}$ \\
\hline Excitation voltage & $\leq 32 \mathrm{~V}$ \\
\hline Relative nonlinearity & $0.1 \%$ \\
\hline Operating temperature range & $-20 \circ \mathrm{C} . . . . .85 \circ \mathrm{C}$ \\
\hline
\end{tabular}

Finally, the forces applied by the actuator to achieve the required displacements were also registered in all cases in order to check any possible stiffness decrease due to slippage in the fixing supports, pulling cable failure, or any other malfunctions source. Load acquisitions for the different tested junction and load steps are shown in Figure 63. It can be observed a linear relation between displacement and applied force (i.e. constant stiffness). Also, it can be noticed that the stiffness in ' $Y$ ' direction is approximately twice the stiffness in ' $Z$ ' direction for both 
junction types, caused by the higher in moment of inertia in this direction. Finally, a slightly stiffer behavior can be observed in junction Type B in relation to Type $A$.

The force recordings will be later used as the input in the detailed FEM model of the junction in order to reproduce equivalent strain states independently of the stiffness difference of fixed supports.
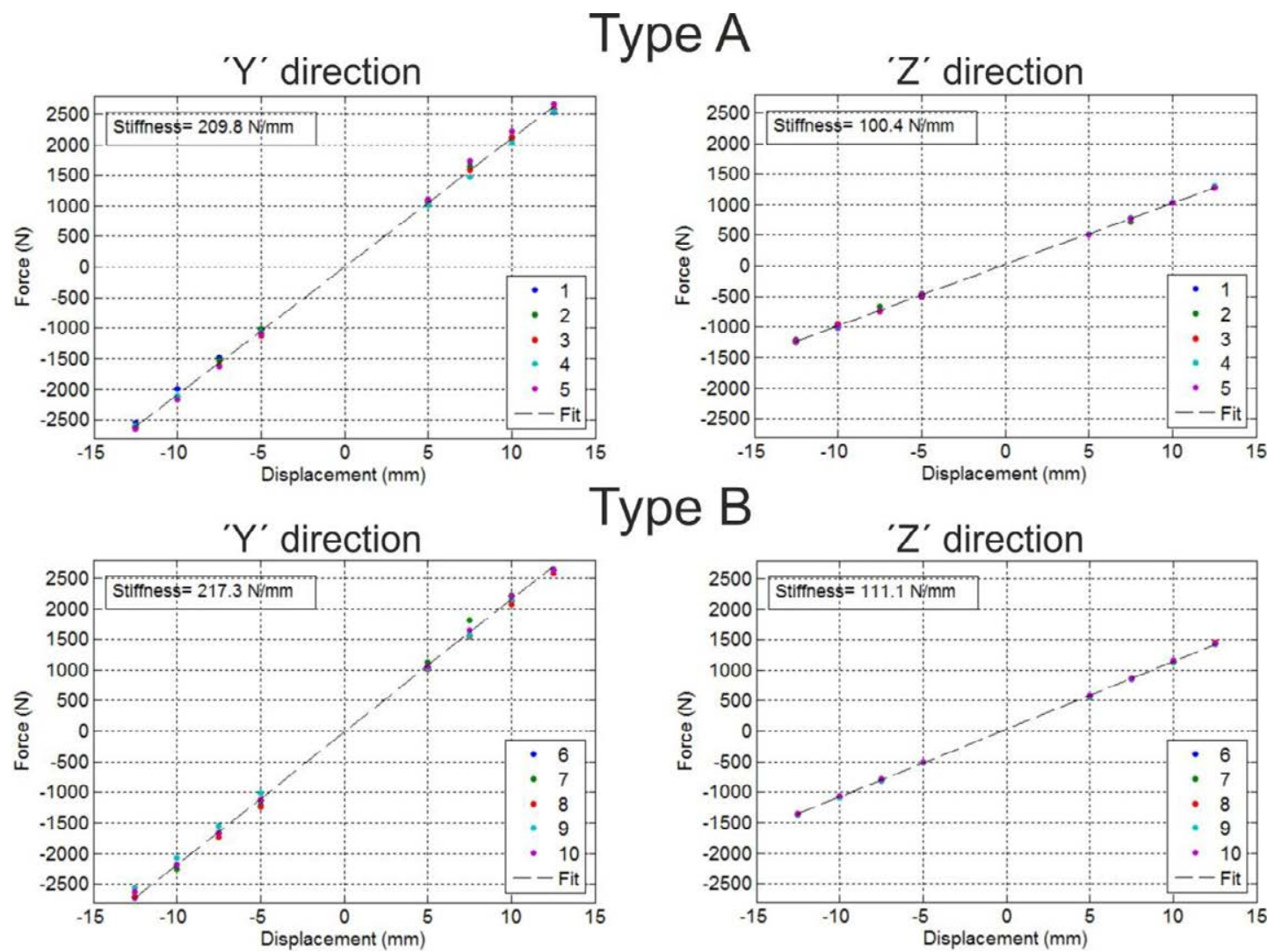

Type B

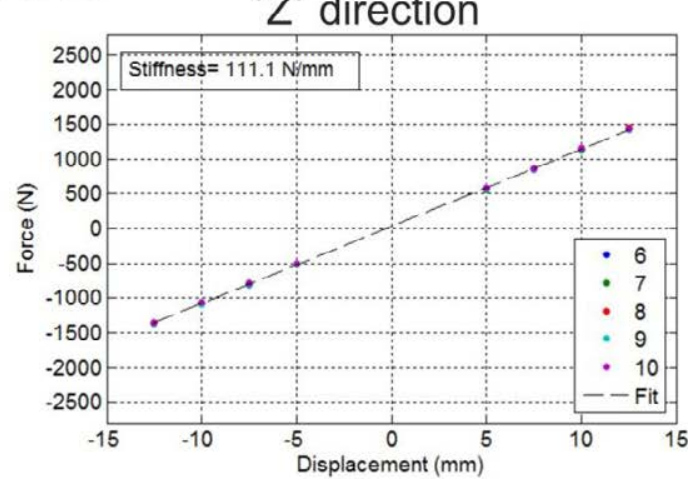

Figure 63 Displacement-Force relation for different junction types and load direction

Given the data of the force or displacement applied, and the geometric and material characteristics, nominal strain or stress values can be easily estimated from the classical strength of materials formulae. Nevertheless, local strain raisings strongly depends on the geometric characteristics of the weld, thus compelling to experimental methods to characterize this behavior. As already stated, a DIC equipment was employed in this study to obtain the strain fields at the vicinity of the junction.

The first step to obtain the strain fields with DIC is to acquire best possible quality images over the region of interest (ROI) of the specimen in unloaded and loaded states. Although the exact locations of the maximum strains are not possible to be predicted in advance, preliminary FEM calculations showed that it will locate in the vicinity of the corner of the junctions (J. Perez et al. 2013). Therefore these zones were chosen as region of interest for the DIC measurements.

As described in Section 3, the surface of the specimen that is going to be measured has to be first prepared with a random contrast pattern. By way of example, Figure 64 shows these patterns on a Type A and type B junction. As it can be noticed, black speckles are randomly distributed over the sprayed matt white layer. 

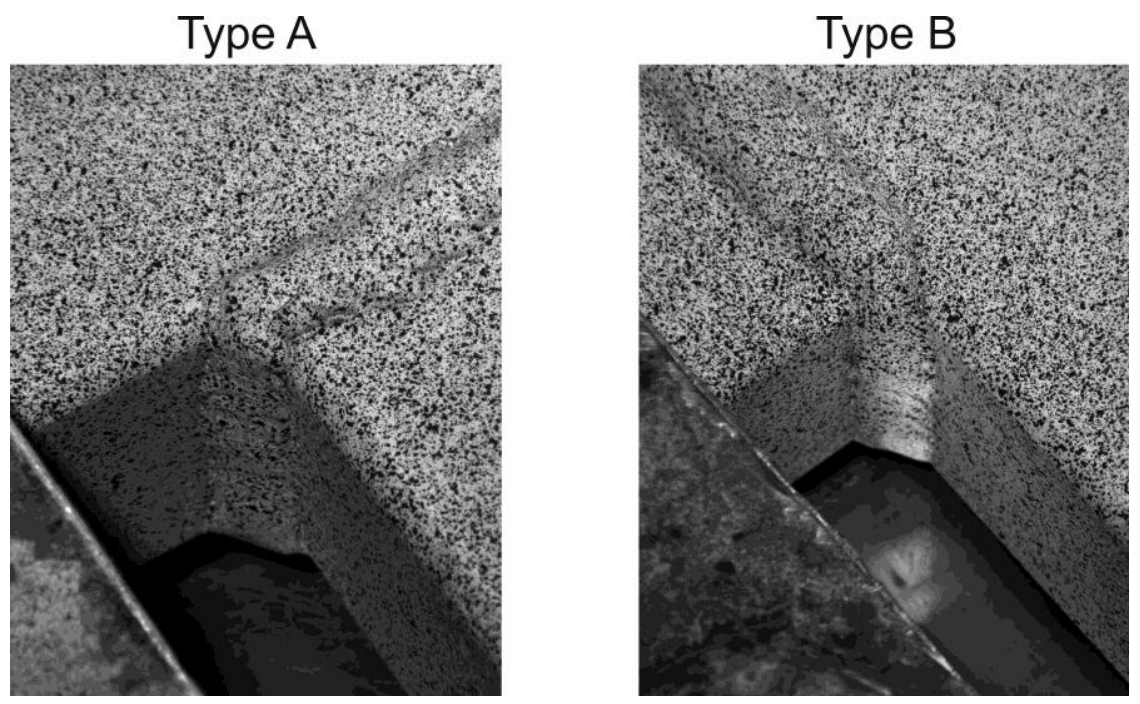

Figure 64 Speckled junctions

The DIC equipment setup is shown in Figure 65. Two cameras were installed to process stereo DIC so that displacements in the 3 dimensions can be accounted. Both cameras should be installed at approximately the same distance from the ROI to avoid issues arising from resolution (i.e. pixel size) differences. Also, extra lighting was applied by means of a spotlight to ensure sufficient and constant lighting over the ROI of the junctions.

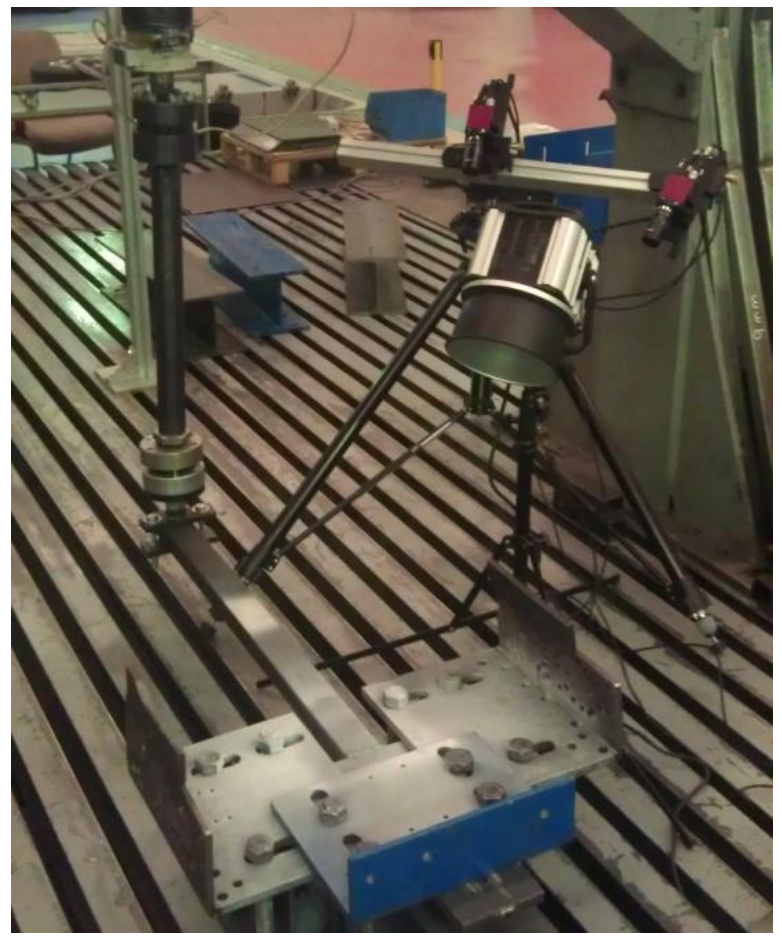

Figure 65 Stereo DIC equipment installation.

Together with each acquired image, analog information about force and displacement were also registered synchronously to later establish the relation between maximum strain and load. 


\subsubsection{IMAGE PROCESSING AND SMOOTHING ERROR}

The commercial software VIC-3D was used to process the acquired images. The processing settings used for image correlation and strain calculation are summarized in Table 14.

Table 14: DIC processing settings

\begin{tabular}{|c|c|}
\hline Subset size & 33 pixels \\
\hline Step Size & 2 pixels \\
\hline Strain Window & 21 data points \\
\hline Strain tensor & Lagrange \\
\hline
\end{tabular}

Once processed all test images, the displacement and strain fields were obtained. By way of example, the following image shows the stain fields obtained for one test image for each junction type and load direction. As it can be observed, a clear tendency is noticed in all cases to locally concentrate the maximum strains on the weld bead toe of the junction corner.

' $Y$ ' direction

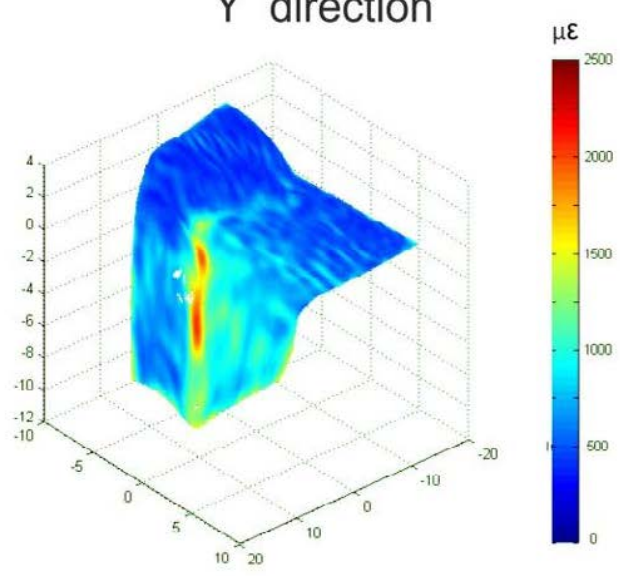

' $Y$ ' direction

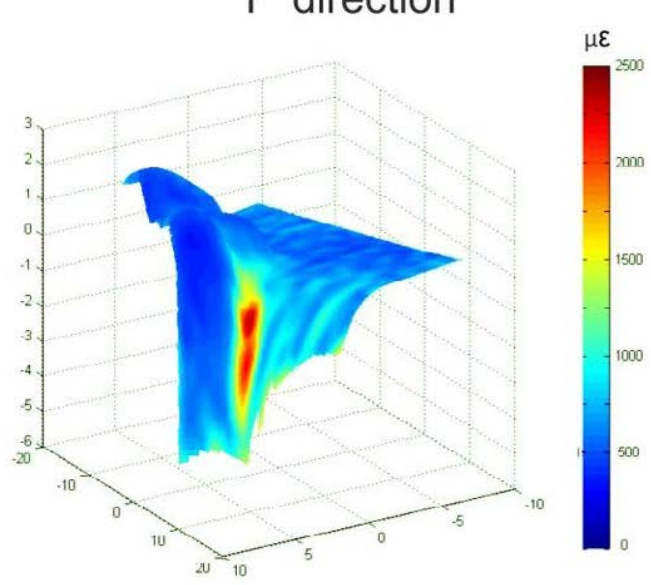

'Z' direction

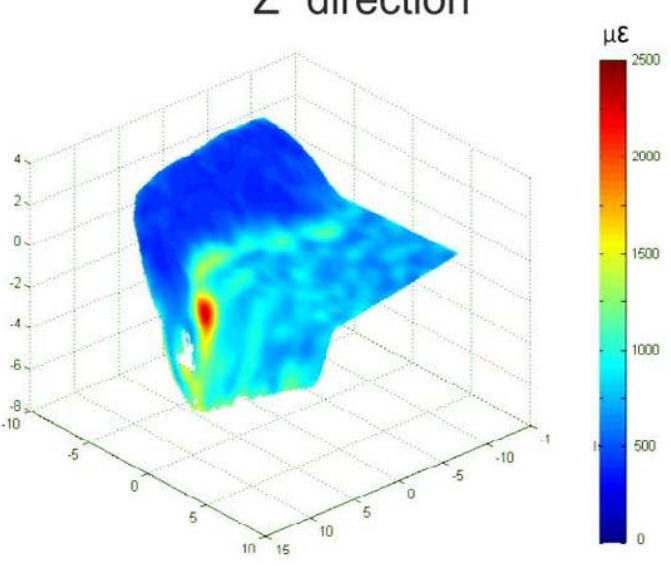

Type B

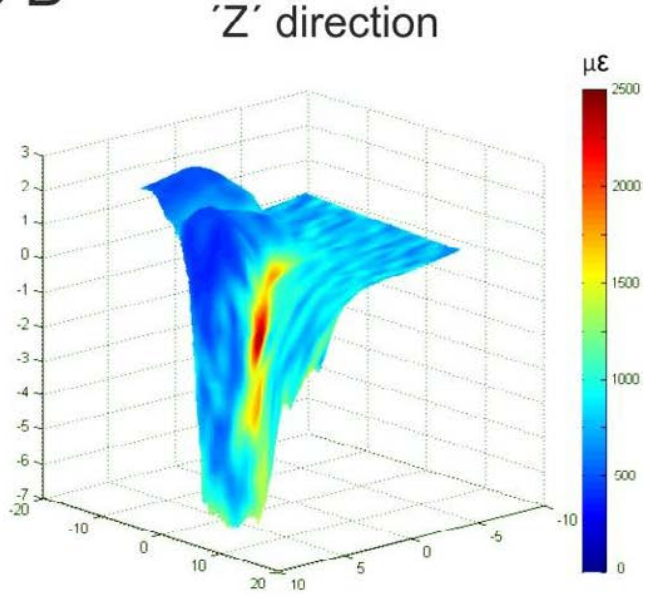

Figure 66 Strain fields obtained with DIC. 
Since the ROI measured has an irregular shape, its orientation with respect to the DIC cameras is not constant. Consequently, the size of the pixel will neither be constant and depend on the distance and orientation of the junction surface with respect to the cameras. The achieved pixel sizes at the location of maximum strains and the consequent VSG are listed in Table 15 and

Table 16 for Type A and Type B junctions respectively.

Table 15: Pixel size and VSG for junctions Type A

\begin{tabular}{|c|c|c|c|c|c|c|c|c|c|c|}
\hline Junction & \multicolumn{2}{|c|}{1} & \multicolumn{2}{c|}{2} & \multicolumn{2}{c|}{3} & \multicolumn{2}{c|}{4} & \multicolumn{2}{c|}{5} \\
\hline & $\begin{array}{c}\text { Pix.Size } \\
(\mu \mathrm{m})\end{array}$ & $\begin{array}{c}\text { VSG } \\
(\mathrm{mm})\end{array}$ & $\begin{array}{c}\text { Pix.Size } \\
(\mu \mathrm{m})\end{array}$ & $\begin{array}{c}\text { VSG } \\
(\mathrm{mm})\end{array}$ & $\begin{array}{c}\text { Pix.Size } \\
(\mu \mathrm{m})\end{array}$ & $\begin{array}{c}\text { VSG } \\
(\mathrm{mm})\end{array}$ & $\begin{array}{c}\text { Pix.Size } \\
(\mu \mathrm{m})\end{array}$ & $\begin{array}{c}\text { VSG } \\
(\mathrm{mm})\end{array}$ & $\begin{array}{c}\text { Pix.Size } \\
(\mu \mathrm{m})\end{array}$ & $\begin{array}{c}\text { VSG } \\
(\mathrm{mm})\end{array}$ \\
\hline $\mathrm{Z}$ & 47.4 & 1.75 & 46.1 & 1.71 & 41.8 & 1.55 & 37.8 & 1.40 & 41.3 & 1.53 \\
\hline $\mathrm{Y}(+)$ & 44.4 & 1.64 & 39.8 & 1.47 & 46 & 1.70 & 38.7 & 1.43 & 39.6 & 1.46 \\
\hline$Y(-)$ & 40.4 & 1.49 & 42.3 & 1.57 & 40.3 & 1.49 & 32.9 & 1.22 & 48.1 & 1.78 \\
\hline
\end{tabular}

Table 16: Pixel size and VSG for junctions Type B

\begin{tabular}{|c|c|c|c|c|c|c|c|c|c|c|}
\hline Junction & \multicolumn{2}{|c|}{6} & \multicolumn{2}{c|}{7} & \multicolumn{2}{c|}{8} & \multicolumn{2}{c|}{9} & \multicolumn{2}{c|}{10} \\
\hline & $\begin{array}{c}\text { Pix.Size } \\
(\mu \mathrm{m})\end{array}$ & $\begin{array}{c}\text { VSG } \\
(\mathrm{mm})\end{array}$ & $\begin{array}{c}\text { Pix.Size } \\
(\mu \mathrm{m})\end{array}$ & $\begin{array}{c}\text { VSG } \\
(\mathrm{mm})\end{array}$ & $\begin{array}{c}\text { Pix.Size } \\
(\mu \mathrm{m})\end{array}$ & $\begin{array}{c}\text { VSG } \\
(\mathrm{mm})\end{array}$ & $\begin{array}{c}\text { Pix.Size } \\
(\mu \mathrm{m})\end{array}$ & $\begin{array}{c}\text { VSG } \\
(\mathrm{mm})\end{array}$ & $\begin{array}{c}\text { Pix.Size } \\
(\mu \mathrm{m})\end{array}$ & $\begin{array}{c}\text { VSG } \\
(\mathrm{mm})\end{array}$ \\
\hline$Z$ & 46 & 1.70 & 40.3 & 1.49 & 40.2 & 1.49 & 41.8 & 1.55 & 34.6 & 1.28 \\
\hline$Y(+)$ & 46.4 & 1.72 & 44.3 & 1.64 & 38.1 & 1.41 & 45.8 & 1.69 & 41.1 & 1.52 \\
\hline$Y(-)$ & 48 & 1.77 & 47.7 & 1.76 & 38.7 & 1.43 & 39.6 & 1.47 & 35.8 & 1.32 \\
\hline
\end{tabular}

As it was already described in section 3, it is of crucial importance to achieve an small virtual strain gauge (VSG) when measuring small and high heterogeneous strain fields as is the present case. Furthermore, it was shown the relation between VSG and smoothing error when calculating peak strain values obtained in J. Perez et al. (2016). Using the mean values of this relation (red line in Figure 37), and the achieved VSG for each test it is possible the determine the resulting smoothing error for each test carried out. Table 17 and Table 18 show these outcomes for junctions Type $A$ and Type $B$ respectively.

Table 17: Smoothing error for junctions Type A

\begin{tabular}{|c|c|c|c|c|c|}
\hline & \multicolumn{5}{|c|}{ Smoothing error $\left(S_{E}\right)$} \\
\hline Junction & 1 & 2 & 3 & 4 & 5 \\
\hline$Z$ & 0.136 & 0.132 & 0.118 & 0.103 & 0.116 \\
\hline$Y(+)$ & 0.126 & 0.111 & 0.131 & 0.116 & 0.109 \\
\hline$Y(-)$ & 0.113 & 0.119 & 0.112 & 0.085 & 0.138 \\
\hline
\end{tabular}


Table 18: Smoothing error for junctions Type B

\begin{tabular}{|c|c|c|c|c|c|}
\hline & \multicolumn{5}{|c|}{ Smoothing error $\left(S_{E}\right)$} \\
\hline Junction & 1 & 2 & 3 & 4 & 5 \\
\hline$Z$ & 0.132 & 0.112 & 0.112 & 0.118 & 0.092 \\
\hline$Y(+)$ & 0.133 & 0.126 & 0.104 & 0.131 & 0.115 \\
\hline$Y(-)$ & 0.137 & 0.137 & 0.106 & 0.109 & 0.096 \\
\hline
\end{tabular}

These values can be then used as compensating factors to estimate the actual maximum strain $\left(\varepsilon_{a c t}\right)$ on each junction and load type.

$$
\varepsilon_{a c t}=\frac{\varepsilon_{D I C}}{\left(1-S_{E}\right)}
$$

And the consequent strain concentration factor can be established as follows:

$$
K_{\varepsilon}=\frac{\varepsilon_{D I C}}{\varepsilon_{n o m}\left(1-S_{E}\right)}
$$

The nominal strain is understood as the strain that would exist at a location if there was not any rising effect. This value could be obtained by extrapolating DIC result sufficiently separated from the strain rising zone. Nevertheless, two limitations were found when trying to perform this operation. On the one hand, the small region of interest used (necessary to obtain a fair small pixel size and VSG) leaves a very small, or sometimes inexistent, portion were the strains could be considered nominal. Additionally, the noise becomes significant for such small strain values, specially for lower loads. Due to these limitations, it was not possible to find consistent nominal strain values from DIC results extrapolation. Consequently, it was used a theoretical approach of the nominal strain calculated as the existing bending strain in relation to the imposed displacement at the location of the maximum strains:

$$
\varepsilon_{n o m}=D \frac{3(h / 2)}{l^{2}}
$$

Where $D$ is the applied displacement amplitude, $l$ is the distance from the point of force application to the maximum strain location, and $h$ is the length of the beam section in the force application direction.

This approach was proven to show great agreement with FEM validated models as will be shown in the next sections.

\subsubsection{STRAIN CONCENTRATION FACTOR RESULTS}

Once extracted all peak strain values from each test, and after the application of the compensating factors, the actual maximum strains on each strain field measured were obtained. Although the strains are caused by flexural loads, a multiaxial strain state arises locally at the 
weld toe due to the irregular shape present at this zone. For this reason, equivalent VonMises strain will be used in the present study in order to take into account the contribution of all components of the strain state. The maximum strains for each junction and load type in relation to the imposed displacements are shown in Figure 67. No significant non-linearity is observed in the evolution of the maximum strains with the displacements. It can also be observed that some junctions present higher strain than others with independence of the load type or displacement applied, which denotes differences in the strain concentration behavior.

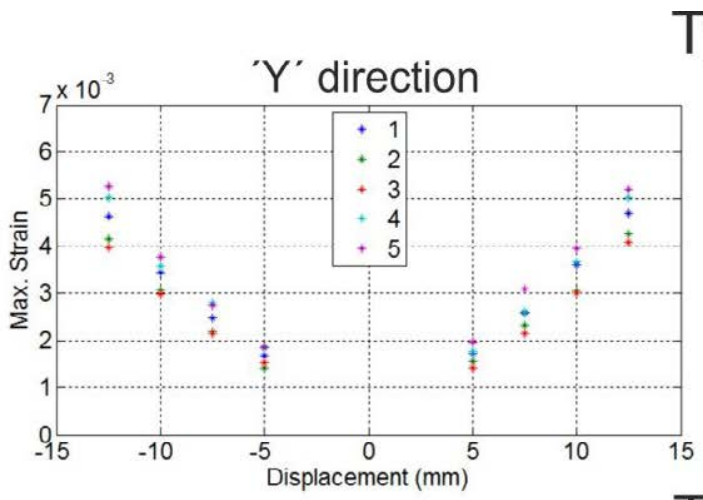

Type A

\section{' $Y$ ' direction}

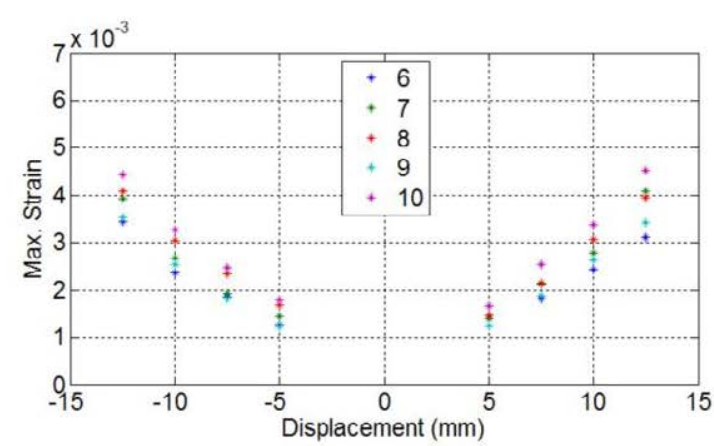

'Z' direction

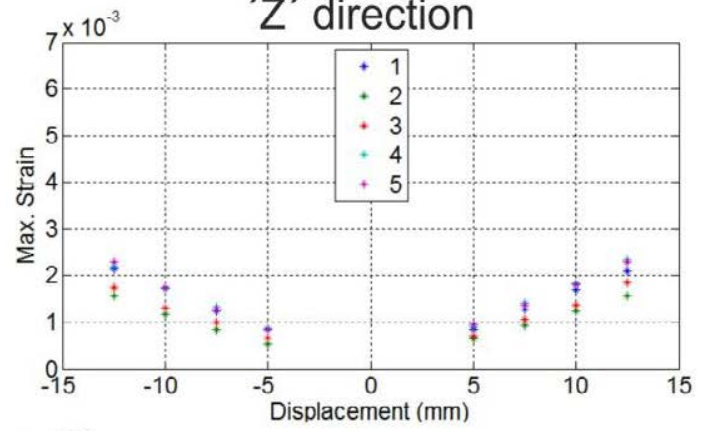

'Z' direction

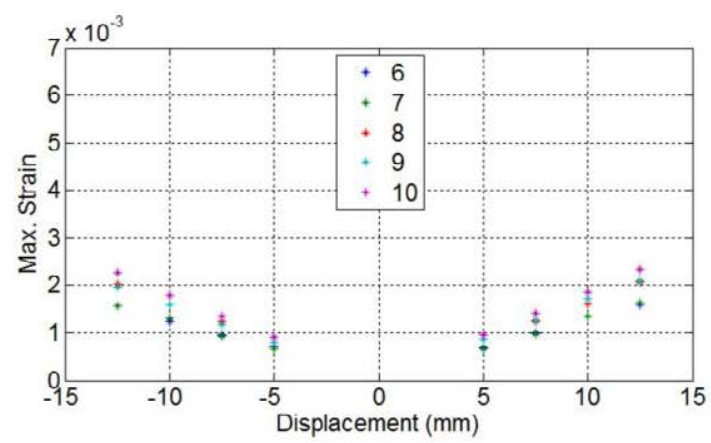

Figure 67 Maximum strains versus imposed displacement.

The strain concentration behavior of the different junctions and load types can be better studied in the following Figure 68, which shows the SCF with respect to the imposed displacements. It can be noticed that, in general terms, when the junctions are loaded in ' $\mathrm{Y}$ ' direction, the SCF values are slightly higher than when loaded in ' $Z$ ' direction, specially for junctions Type A. Additionally, no significant difference of the SCF is appreciated with the increasing displacement when load is applied in ' $Z$ ' direction. On the contrary, for both types of junctions it is noticed a slight increment of the SCF for displacements of $\pm 12.5 \mathrm{~mm}$ when the load is applied in ' $Y$ ' direction. It is most likely caused by the effect of local non linear behavior of the material at the strain concentration zone. 

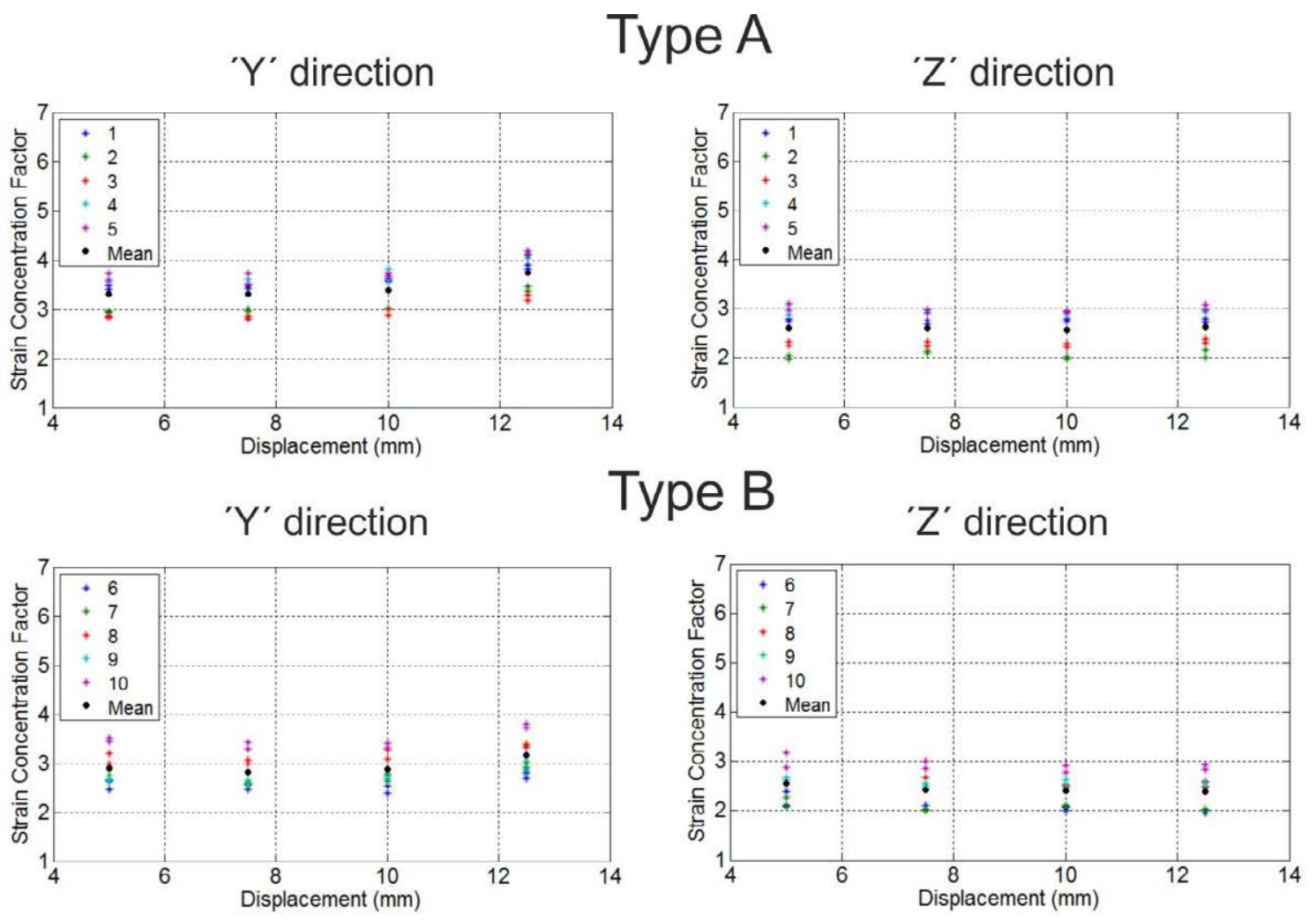

Figure 68 SCF versus imposed displacement.

To further study the evolution of the SCF with the displacement increment, it was decided to evaluate the SCF increment $\left(\Delta K_{e}\right)$ with respect to the one measured at a displacement of 5 $\mathrm{mm}$ (where elastic behavior can be assumed), for each junction type (i), load direction (j), and imposed displacement ( $n$ ). The operation for obtaining the SCF increment is represented in the following equation:

$$
\Delta K_{\varepsilon(i, j, n)}=K_{\varepsilon(i, j, n)}-K_{\varepsilon(i, j, \pm 5 m m)}
$$

It can be deduced from the equation that all $\Delta K_{e}$ will take null values for imposed displacements of $\pm 5 \mathrm{~mm}$, since they will equal the subtracting component. Therefore, they were removed from the SCF study.

The values thus obtained enables to assess the impact of the strain level over the SCF in an isolated manner. Results are shown in Figure 69 and Figure 70, plotted with respect to the maximum and nominal strains respectively. It is noticed an increasing tendency of the SCF with the increasing strains of the junction. This tendency also shows to be independent load direction, or junction type, depending uniquely on the response of the material to the strain level, which is in turn dictated by the material flow characteristics. Consequently, it is very likely that the power behavior of the SCF increment is in close relation with the increment of the plastic deformation when increasing the total strain, which is known to show great agreement to power functions as it was shown in sections 3 and 5. Furthermore, other well known theoretical approaches to the evolution of the SCF with the plastic strain (H. Neuber, 1961 or G. Glinka, 1987) also propose power relations based on the flow curve data of the material. 


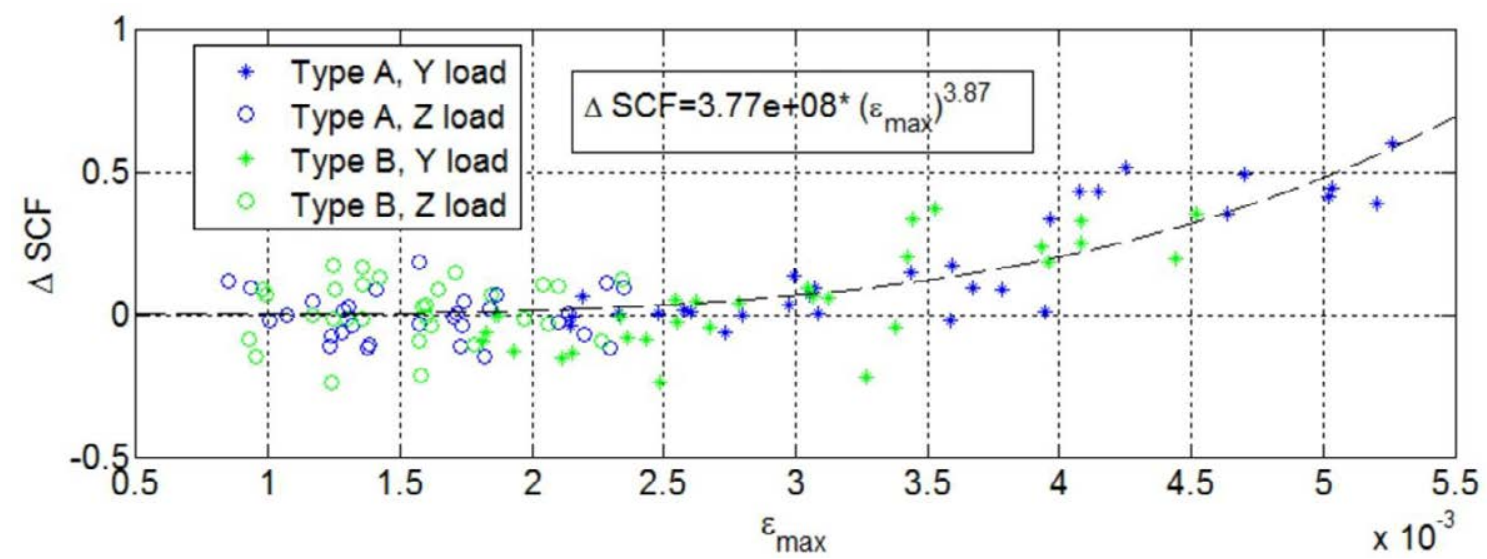

Figure 69 SCF increment tendency in relation to the maximum strain .

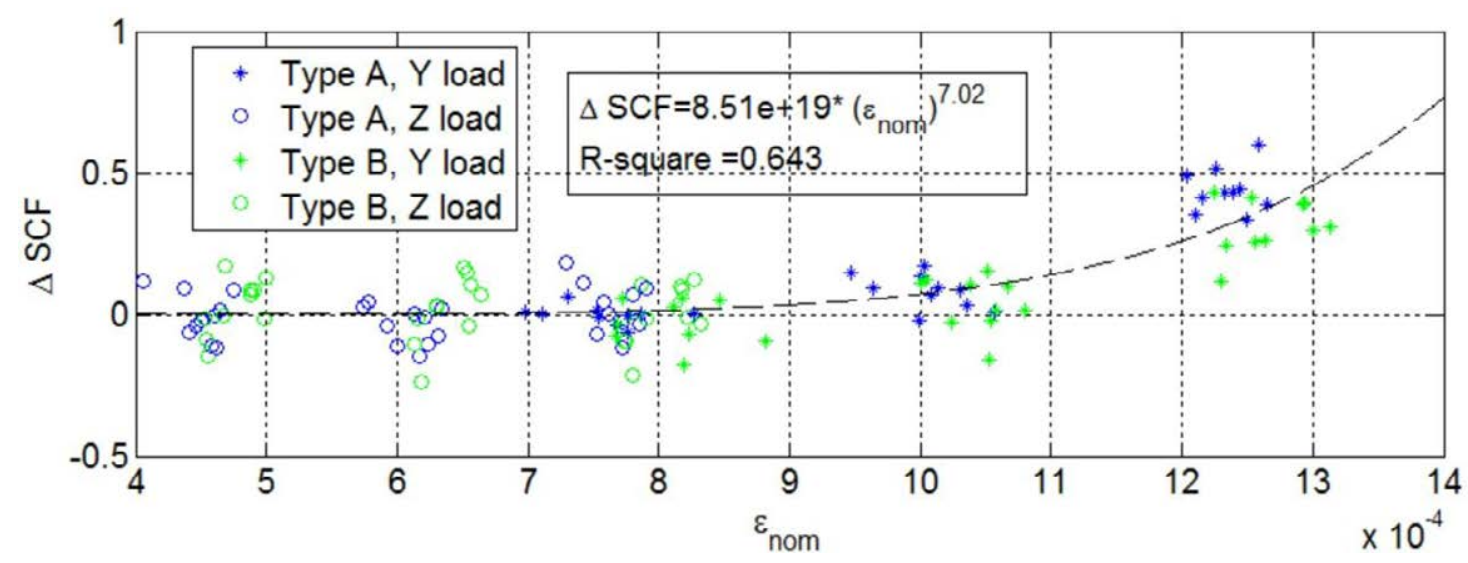

Figure 70 SCF increment tendency in relation to the nominal strain.

The regression function obtained will be later employed during the application of the fatigue life prediction methodology as a correcting factor of the SCF. It should be noted that, although Figure 69 has more physical meaning given that peak strains actually govern nonlinear behavior of the material, it is not possible to know in advance this peak value in a LPTV modeled with beam type elements as will be shown later. Therefore, the power regression brought in Figure 70 will be used for this correction.

In any case, as it will be seen later, such high strain values correspond to high strain fatigue cycles (i.e. low fatigue life), that are not very common to occur during regular operation of common LPTV structure designs.

An additional characteristic that can be extracted from Figure 68 is the significant scatter of the SCF values, which has a direct lowering effect on the R-square of the regression (0.643). This scattering does not show appreciable variation with the junction type, load direction or displacement level. In this scenario, the only difference between same junction types subjected to same load direction and level is the local geometry of the weld bead toe, and thus it can be considered as the main cause of the SCF scatter. In relation to this, it is also observed that a junction that shows a high SCF, keeps this behavior regardless the load direction or displacement level, and vice versa. 
To study this phenomenon with more clarity, next Figure 71 shows the difference between the SCF and its mean value calculated for each junction type (i), load direction (j), and imposed displacement ( $n$ ) (black dots in Figure 68), according to equation (1.99). It is presented in the figure in relation to the maximum strain. This allows evaluating the dispersion of the SCF in with independently of the SCF level of each test.

$$
\operatorname{Dev}\left(K_{\varepsilon(i, j, n)}\right)=K_{\varepsilon(i, j, n)}-\frac{\sum_{k=1}^{k(i, j, n)} K_{\varepsilon(i, j, n)}}{k(i, j, n)}
$$

Where ' $k$ ' is the number of junctions test of a specific type, load directions and imposed displacement level.

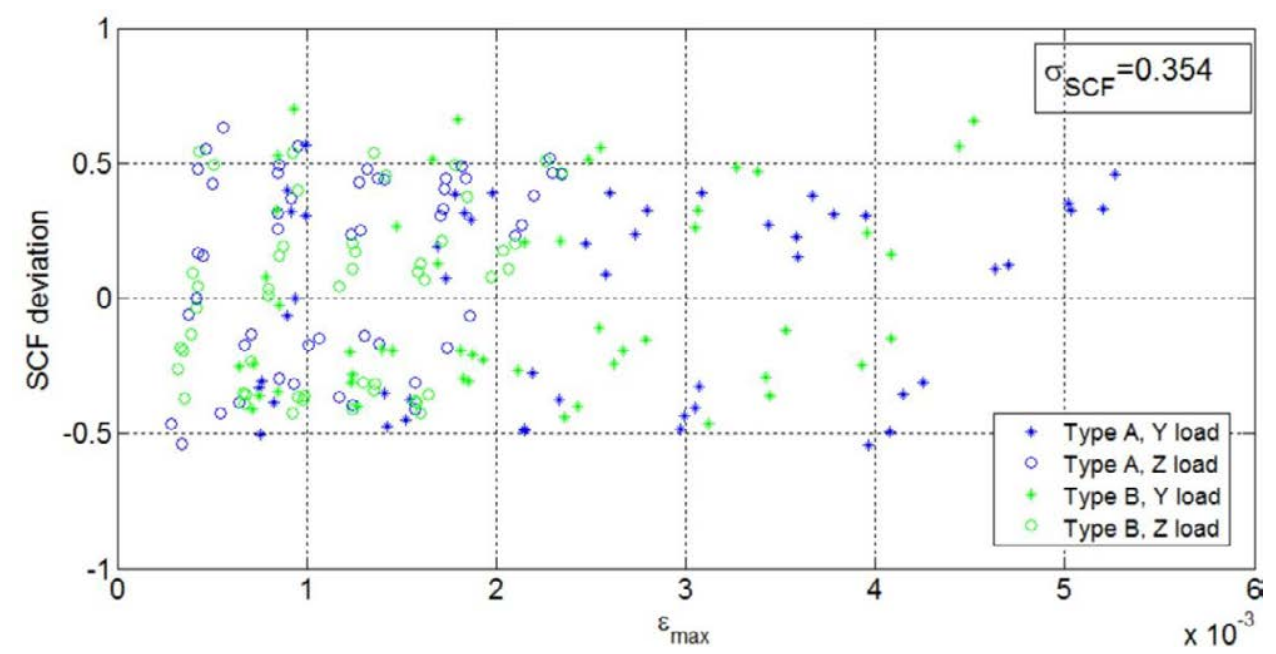

Figure 71 SCF scatter.

It is clearly observed that there is no tendency on the scatter behavior of the SCF regardless the junction type, load direction, displacement level or maximum strain. The overall standard deviation of the SCF results is 0.354 . This value will be used on the statistical operations of the SCF that will be carried out on the subsequent sections.

\subsection{FATIGUE RESISTANCE OF RECTANGULAR TUBE BEAM WELDED JUNCTIONS: STRAIN-LIFE CURVE}

As previously mentioned, the properties of the material are modified during the welding process. Also, it was shown an important influence of geometry of the weld bead on the SCF. In this scenario, experimental procedures become crucial for obtaining reliable fatigue characteristics of LPTV welded junctions.

This section describes the research actions carried out to experimentally determine the parameters that compose the e- $\mathrm{N}$ fatigue life curve presented in Section 3. 


\subsubsection{TEST CONFIGURATION: OBTENTION OF MAXIMUM STRAIN VS LIFE DATA}

The characteristics of the welded junctions, and the clamping configuration coincide with the one used in the previous section for the SCF study, so no further details will be given in this regard.

A total of 30 welded junctions were tested under flexural load in ' $Z$ ' direction. The objective of each test is to establish a strain amplitude versus life point in the $\varepsilon-\mathrm{N}$ diagram. Therefore, it is necessary, on the one hand, to estimate the strain amplitude and its plastic and elastic components in each test. On the other hand, it is necessary to establish a failure condition (based on stiffness decrease of the junction) and record the number of cycles until this failure occurs.

\subsubsection{Strain amplitude determination}

As can be observed from the specimen clamping (Figure 60), the four corners of the junction closer to the load application point will equally act as strain concentrators. Hence, it is unknown in advance which corner will tend to concentrate higher strains and therefore propagate the fatigue crack. As a consequence, it is not possible to take the maximum strain value obtained in the previous section, since it only considers one of the corners, and thus would understate the actual maximum strain in the complete junction, leading to nonconservative results.

From the characterization of the statistical dispersion of the maximum strains, it is possible to determine the maximum strain value statistically expected over all four corners $(p)$ for each junction type (i), load direction (j), and load level (n). Given that $K_{\varepsilon(i j, k)(\max )}$ is the maximum of the SCF of each of the four corners of the junctions:

$$
K_{\varepsilon(i, j, n)(\max )}=\max \left(K_{\varepsilon(i, j, n)(p)}\right) \quad \forall p=(1, \ldots, 4)
$$

The cumulative probability distribution of the maximums $\left(F\left(K_{\varepsilon(i, j, k) \text { max }}\right)\right)$ can be easily determined as the nth power (number of samples, in this case $p=4$ ) of the cumulative probability distributions of the SCF in study:

$$
F\left(K_{\varepsilon(i, j, n)(\max )}\right)=F\left(K_{\varepsilon(i, j, n)}\right)^{p}
$$

Consequently, the expected value (or average, $\left.\left.\left(F\left(K_{\varepsilon(i, j, k) \text { max }}\right)\right)=0.5\right)\right)$ of the maximums corresponds to a value of $\left(0.5^{(1 / 4)}\right)$, which is in turn 0.9982 standard deviations of the mean value of $K_{\varepsilon(i, j, n)}$. Obtaining:

$$
K_{\varepsilon(i, j, n)(\max )}=\bar{K}_{\varepsilon(i, j, n)}+0.9982 \sigma_{S C F}
$$

The obtained expected values for each junction type, load direction, and load level ( $n$ ) are summarized in Table 19. 
Table 19: expected SCF values

\begin{tabular}{|c|c|c|c|c|}
\hline & \multicolumn{2}{|c|}{ Type A } & \multicolumn{2}{c|}{ Type B } \\
\hline Disp $(\mathrm{mm})$ & $'{ }^{\prime}$ & ${ }^{\prime} Z^{\prime}$ & $'{ }^{\prime}$ & $'{ }^{\prime}$ \\
\hline $\pm 5 \mathrm{~mm}$ & 3.67 & 2.96 & 3.25 & 2.90 \\
\hline $\pm 7.5 \mathrm{~mm}$ & 3.66 & 2.96 & 3.17 & 2.78 \\
\hline $\pm 10 \mathrm{~mm}$ & 3.75 & 2.92 & 3.24 & 2.76 \\
\hline $\pm 12.5 \mathrm{~mm}$ & 4.10 & 2.99 & 3.57 & 2.74 \\
\hline
\end{tabular}

Finally, according to eq. (1.64) and eq. (1.97), the total strain amplitude of the fully reversed fatigue cycles applied during the experiments can be determined as follows:

$$
\varepsilon_{\max }=K_{\varepsilon(i, j, n)(\max )} D \frac{3(h / 2)}{l^{2}}
$$

\subsubsection{Obtaining the number cycles to failure}

As it was described before, loads were applied in the form of displacement controlled cycles. It is known that fatigue experiments can entail the application of hundreds of thousands or even millions of cycles, and thus can be significantly extend over time.

A direct way to shorten the test period is by increasing the load frequency. Nevertheless, it has to be taken into account that the frequency must be sufficiently low with respect to the natural frequency of the components that does not influence on its structural response.

In order to estimate the frequencies of the first modes of vibration of the clamped junctions and thus establish a test frequency upper limit, a finite element model modal calculation was performed for both junctions types. In both cases, the displacements of the junction were constrained at the areas in contact the clamping assembly (Figure 60). The deformed shapes and frequencies obtained are shown in (Figure 72). Both junction types show natural frequencies slightly over $50 \mathrm{~Hz}$, so well lower frequencies should be used for the fatigue tests.

It should be also noted that the simulations assumes the zones of fixed constrains as infinity rigid, whereas as the actual assembly the clamping components have high, but finite stiffness. Consequently, the simulation is based in an overall stiffer assembly than reality, and thus the real modes of vibration expected in actual tests would be slightly lower. 


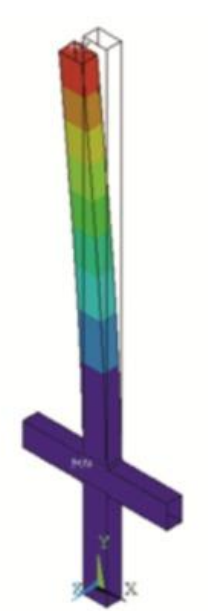

$50.963 \mathrm{~Hz}$

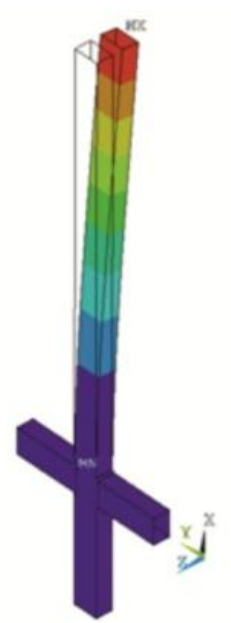

$53.570 \mathrm{~Hz}$

\section{Type A}

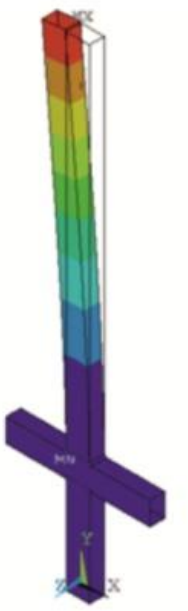

$70.541 \mathrm{~Hz}$
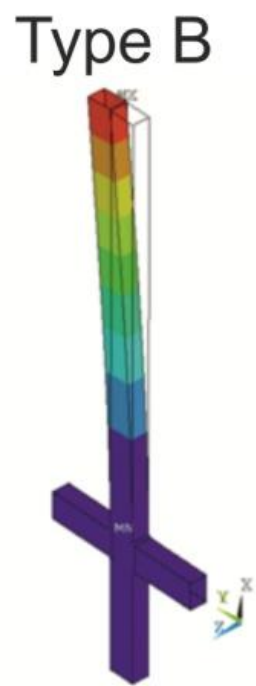

$74.741 \mathrm{~Hz}$

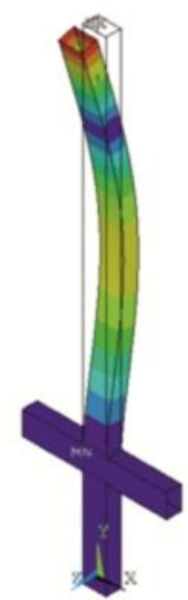

$314.34 \mathrm{~Hz}$

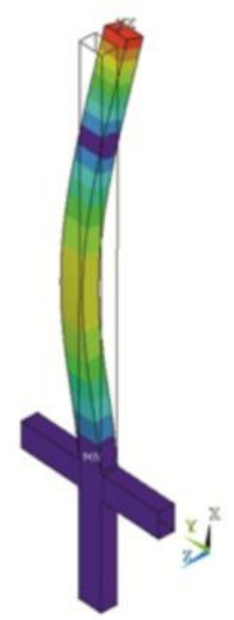

$325.20 \mathrm{~Hz}$

Figure 72 Modal frequencies of tested junctions.

Another restriction on the test frequency arises from to the difficulties of the hydraulic system to control high displacement amplitudes at high frequencies due to the oil flow rate limitations. This effect was visible at frequencies of $6-7 \mathrm{~Hz}$ for imposed displacement amplitudes of $12.5 \mathrm{~mm}$, so it was decided to use a constant test frequency of $4 \mathrm{~Hz}$ independently of the displacement amplitude. This frequency is low enough with respect to the natural frequencies of the junctions that its effect on the structure response can be neglected.

Once decided the test frequency, the last step is to establish a failure criteria at which the hydraulic system will halt. This criterion can be established from the force and displacement data of the hydraulic actuator. Given that the fatigue test is displacement controlled, the load is expected to decrease as fatigue crack advances until the resistance section becomes so small that cannot stand once single cycle.

During the last stage of the crack propagation, the growth rate becomes significantly high, and the stiffness decreases rapidly with every fatigue cycle. The hydraulic system was configured to stop when the force dropped to $50 \%$ from the initial value, or if the relative force decrease in 
two consecutive cycles exceeds 5\%. It was observed that these conditions corresponds to the last stage of the crack propagation, and the crack growth rate is so high in this point that the junction is expected to completely cut off in a few cycles.

By way of example next Figure 73 and Figure 74 show the evolution of the force and displacement during a fatigue test for type $A$ and type $B$ junctions respectively, as well as the cracked component.

Type A, Disp=10mm
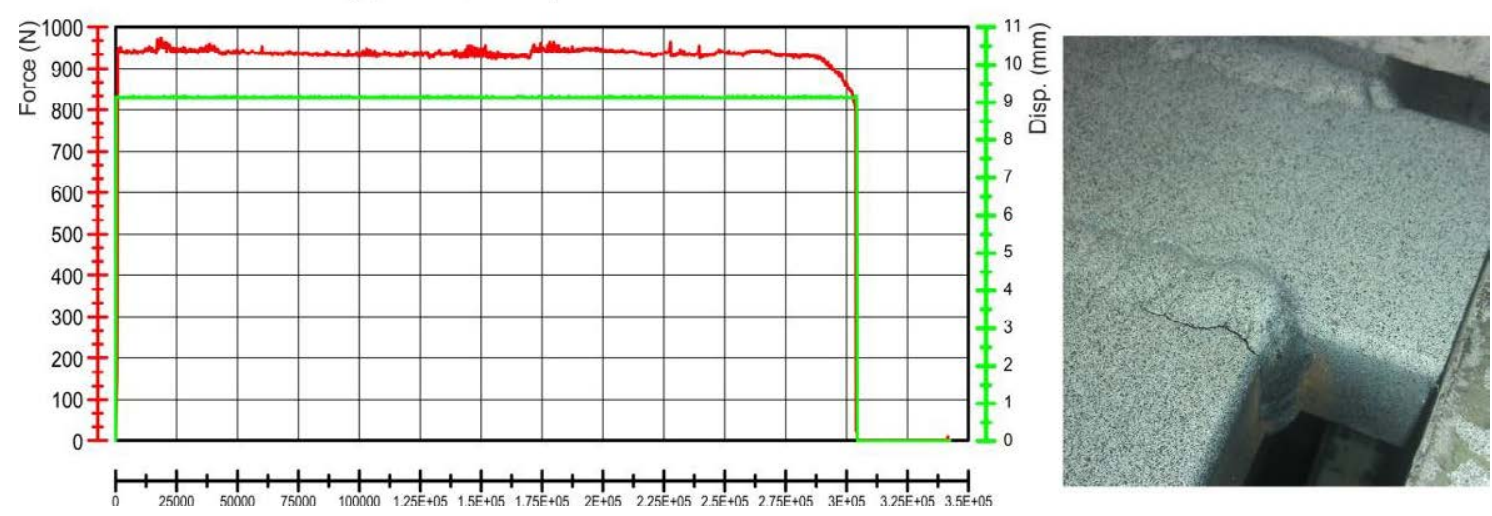

Figure 73 Fatigue test on a Type $A$ junction.

Type B, Disp=10mm
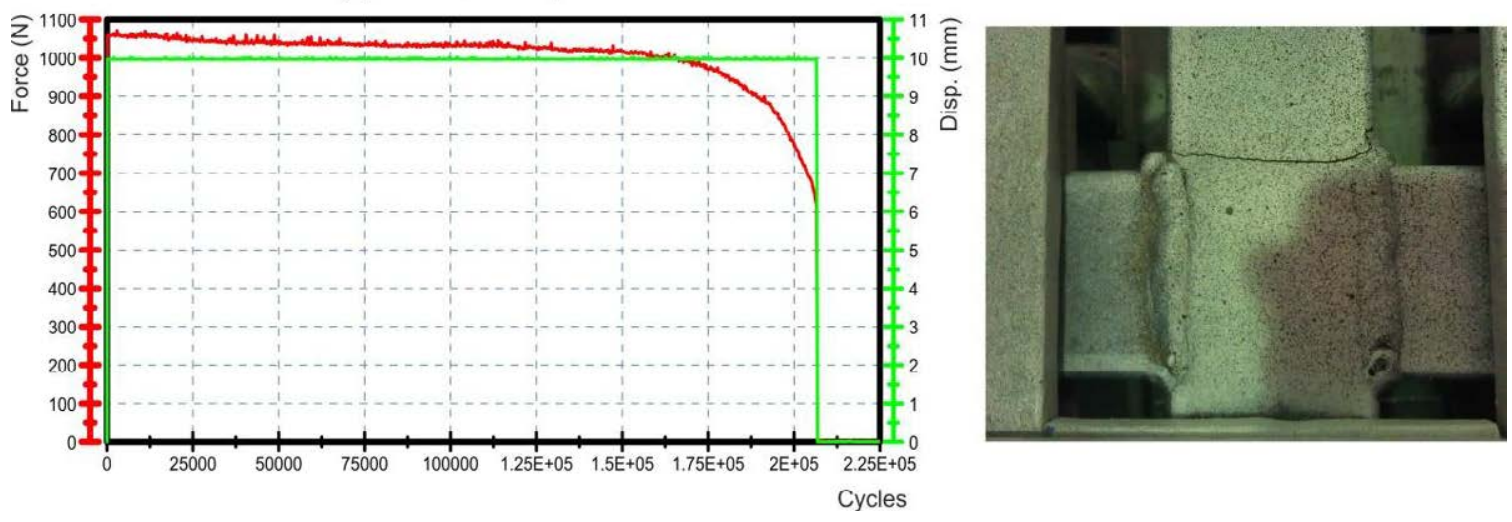

Figure 74 Fatigue test on a Type $B$ junction.

\subsubsection{TEST RESULTS: STRAIN-LIFE CURVE PARAMETERS}

Once all the fatigue test have been carried out, and the criteria for strain amplitude and number of cycles are set in Sections 5.3.1.1 and 5.3.1.2 respectively, it is possible to obtain one point in the $\varepsilon-N$ curve for each of the welded junctions tested. The following Table 20 summarizes the test conditions and results obtained. It can be observed that although a total of 30 junctions were tested, 5 of them were discarded since they produced unexpected outcomes, principally due to instabilities on the clamping system and the hydraulic cylinder. Therefore, the table gathers the results for the 25 valid tests. 
Table 20: Fatigue test conditions and results

\begin{tabular}{|c|c|c|c|c|c|}
\hline Junction No. & Type & $\Delta \varepsilon_{t}$ & $\Delta \varepsilon_{e}$ & $\Delta \varepsilon_{p}$ & Reversals (2N) \\
\hline 1 & $A$ & 0.0022 & 0.0013 & 0.0008 & 140000 \\
\hline 2 & $A$ & 0.0019 & 0.0013 & 0.0006 & 320000 \\
\hline 3 & $A$ & 0.0014 & 0.0011 & 0.0003 & 910000 \\
\hline 4 & A & 0.0012 & 0.0011 & 0.0001 & 3544000 \\
\hline 5 & $\mathrm{~A}$ & 0.0016 & 0.0012 & 0.0004 & 526000 \\
\hline 6 & $A$ & 0.0017 & 0.0012 & 0.0005 & 640000 \\
\hline 7 & $A$ & 0.0014 & 0.0011 & 0.0003 & 800000 \\
\hline 8 & $A$ & 0.0017 & 0.0012 & 0.0005 & 362000 \\
\hline 9 & $A$ & 0.0018 & 0.0012 & 0.0006 & 580000 \\
\hline 10 & $A$ & 0.0018 & 0.0012 & 0.0006 & 336000 \\
\hline 11 & $A$ & 0.0014 & 0.0011 & 0.0003 & 860000 \\
\hline 12 & $\mathrm{~B}$ & 0.0018 & 0.0013 & 0.0005 & 370000 \\
\hline 13 & $A$ & 0.0022 & 0.0013 & 0.0009 & 98000 \\
\hline 14 & $A$ & 0.0022 & 0.0013 & 0.0009 & 102000 \\
\hline 15 & $\mathrm{~B}$ & 0.0023 & 0.0013 & 0.0010 & 86000 \\
\hline 16 & $\mathrm{~B}$ & 0.0018 & 0.0013 & 0.0005 & 500000 \\
\hline 17 & $B$ & 0.0018 & 0.0013 & 0.0005 & 210000 \\
\hline 18 & $B$ & 0.0014 & 0.0011 & 0.0003 & 1590000 \\
\hline 19 & $A$ & 0.0016 & 0.0012 & 0.0004 & 370000 \\
\hline 20 & $A$ & 0.0016 & 0.0012 & 0.0004 & 232000 \\
\hline 21 & $\mathrm{~B}$ & 0.0014 & 0.0011 & 0.0003 & 1260000 \\
\hline 22 & $A$ & 0.0014 & 0.0011 & 0.0003 & 1180000 \\
\hline 23 & $A$ & 0.0013 & 0.0011 & 0.0002 & 1310000 \\
\hline 24 & $B$ & 0.0018 & 0.0013 & 0.0005 & 182000 \\
\hline 25 & B & 0.0013 & 0.0011 & 0.0002 & 1560000 \\
\hline
\end{tabular}

Applying separate linear least squares fittings to the elastic and plastic strain components of the deformation amplitude as described in Section 3, it is possible to obtain the four fatigue parameters that compose the Coffin-Manson relation. Although very low plastic deformation data is usually recommended to be discarded from the linear fit of the plastic strain component, it was found that the best fit to experimental data was achieved when using all experimental data for both curve fittings. Therefore, both fits are performed based on all valid data obtained from the experimental data.

The obtained parameters are shown in Table 21. As would be expected, both elastic and plastic slopes in the logarithmic scales $(b$ and $c$ ) are negative. This slope is notably more pronounced in the case of the plastic strains (-0.063 versus -0.374$)$, evidencing that for high cycle fatigue, elastic deformations are predominant, and thus dictate fatigue failure. On the opposite, the intercept $(\mathrm{N}=0)$ is significantly higher in the plastic deformations than in the elastic ones $\left(\sigma_{f^{\prime}} / E=0.0027\right.$ versus $\left.\varepsilon_{f^{\prime}}=0.063\right)$. Therefore, ductility of the material will dominate the fatigue behavior in low cycle fatigue.

Table 21: Coffin-Manson equation parameters

\begin{tabular}{|c|c|c|c|}
\hline $\boldsymbol{\sigma}_{f^{\prime}} / \boldsymbol{E}$ & $\boldsymbol{b}$ & $\boldsymbol{\varepsilon}_{f^{\prime}}$ & $\boldsymbol{c}$ \\
\hline 0.0027 & -0.063 & 0.063 & -0.374 \\
\hline
\end{tabular}


By substituting the obtained values in the Coffin-Manson relation, the final expression of the e-N curve can be written as follows:

$$
\frac{\Delta \varepsilon_{t}}{2}=0.0027\left(2 N_{f}\right)^{-0.063}+0.063\left(2 N_{f}\right)^{-0.374}
$$

This expression is represented graphically in Figure 75, over which the experimental results of the tests junctions are plotted. The graph ratifies the aforementioned decreasing influence of plastic deformations as the number of cycles to failure increase. It can be also observed that no significant differences in fatigue resistance are noticed between Type $A$ and Type B junctions.

It is also observed that the transition fatigue life $\left(2 N_{f}\right)$ is around $4 \mathrm{e}^{4}$ reversals. This points is out of the range of fatigue life covered in the tests (from 9e4 to 3.5e6). It should be pointed that the curve fitting can present poorer precision out of this range, and fatigue data should be taken with care in this situation. In any case, usual operating loads are very rare to incur in such low fatigue lives in usual LPTV designs as it will be seen later.

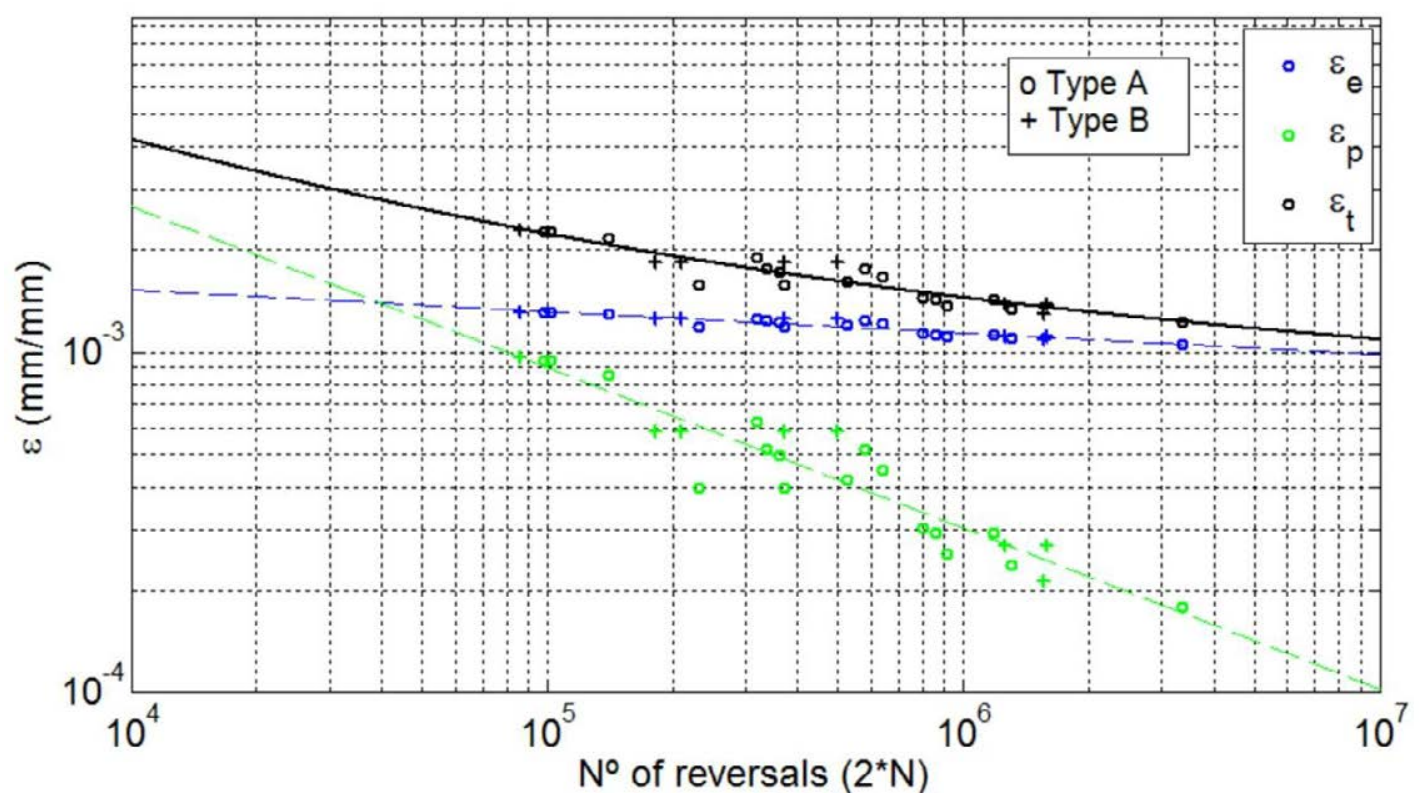

Figure 75 Strain-life curve.

Finally, it should be taken into account that the fatigue tests have been conducted in zero mean strain conditions. In order to account the contribution of nonzero mean strains that might arise in actual load cycles in LPTV structures, the eq. (1.104) should be transformed in the following eq. (1.105). This expression minimizes the elastic component of fatigue resistance by the mean strain according to the proposal of Morrow (1968) described in Section 3.

$$
\frac{\Delta \varepsilon_{t}}{2}=\left(0.0027-\varepsilon_{m}\right)\left(2 N_{f}\right)^{-0.063}+0.063\left(2 N_{f}\right)^{-0.374}
$$




\section{VALIDATION OF A FINITE ELEMENT JUNCTION MODEL}

In the last sections, it has been evidenced the costly process to characterize the strain concentration behavior of two different welded junctions types, each in two different load directions. Taking into account that LPTV structures use thousands of different junction types, an experimental characterization of the strain concentration behavior over all these junctions becomes an hardly feasible option.

To overcome this issue, the use of finite element techniques to calculate a wider range of welded junctions presents itself as an clear alternative. For this purpose though, it is necessary to perform a previous validation process in order to ensure that the models behave in accordance to reality.

The present section shows the construction and validation process of a detailed FEM model of the welded junctions previously studied experimentally.

\subsection{FINITE ELEMENT MODEL CONFIGURATION}

As already pointed in section 3 , it is a common practice to use simplified modeling techniques of beam like structural models. However, this is not a feasible option for the present objective since the junction strain concentration behavior was proven to strongly depend on the geometrical details in the vicinity of the welded bead, which cannot be accounted using simplified models. Even detailed models that does not account in a very precise manner of the weld bead geometry were proven to show important deviations from experimental results (J.A. Pérez et al.,2013).

In this scenario, a profound study and precise implementation of these geometrical details on the FEM model becomes crucial to obtain satisfactory outcomes. Other configuration options that must be consciously accounted are the material model, meshing characteristics (especially important at the weld bead corner), and boundary conditions. It can be noticed that all these factors are the ones that condition the stiffness matrix of the model, and therefore dictates its response.

\subsubsection{GEOMETRY}

The rectangular section of the welded beams was modeled as shown in Figure 76. It can be noticed that it considers the blend radius in the section corners. This radius, although might have a minor contribution to the global stiffness of the model, has a notable influence on the geometry of the welded junction corner, which is where the highest strain concentrations are located as already shown experimentally. Consequently, its inclusion on the model is of crucial importance for the present purpose. 


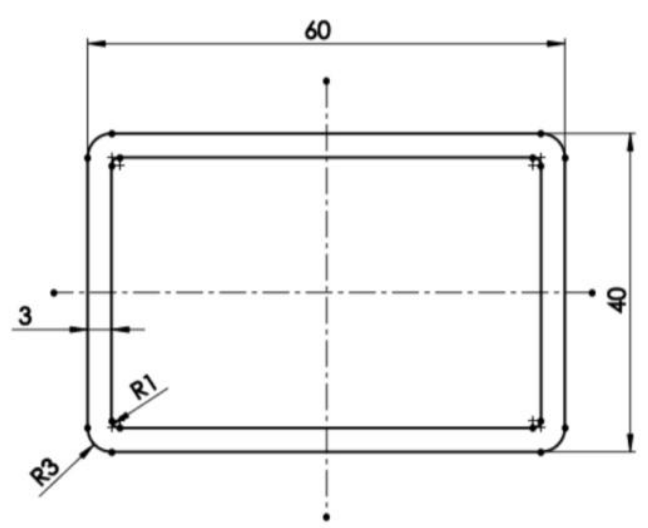

Figure 76 Beam rectangular profile section.

Regarding the welded bead geometry, it is not as constant and easy to determine as the cross section. Its variability is also the main cause of the strain concentration scatter observed in the experimental tests. Therefore, peak strain values obtained from the FEM model will be highly sensitive to this geometry.

For the characterization of the geometry, the shape of the weld bead was supposed as a circumference arc that protrudes from the base profile by a height of ' $\mathrm{H}$ ' along a total length of ' $\mathrm{B}$ ', and has an accordance radius of ' $\mathrm{R}$ ' as it is schematized in Figure 77.

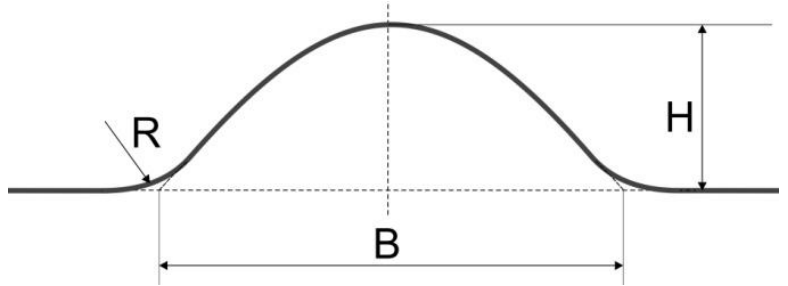

Figure 77 Weld bead section parameters.

From the junctions tested by means of the DIC equipment, it is possible to obtain the position of each data point along the measured field, which in turn enables obtaining the shape of the weld bead in this area. In total, profiles of ten sections belonging to different welded junctions were studied to obtain the parameters mentioned. The following Figure 78 show the shape of this sections, as well as the average values of each parameter. It can be noticed the significant variability among profiles.

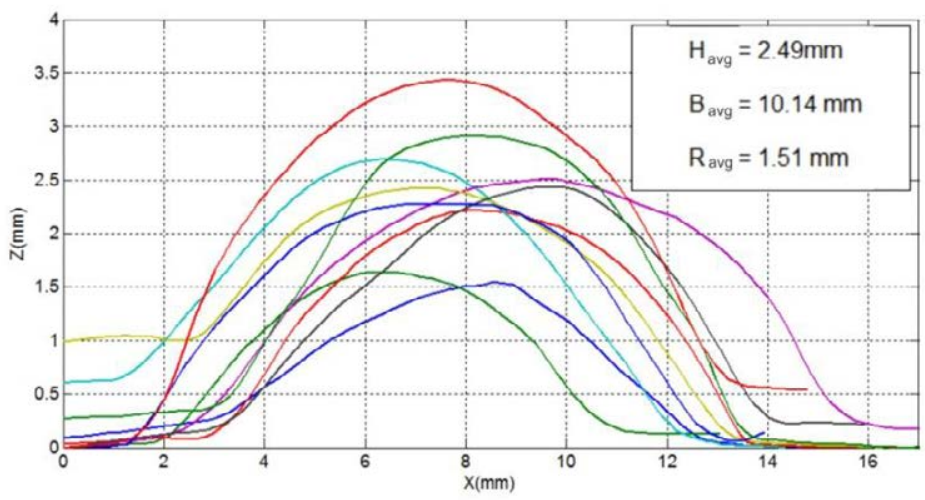

Figure 78 Weld bead section measurements. 
The rounded values of the obtained averages $H=2.5 \mathrm{~mm}, B=10 \mathrm{~mm}$, and $\mathrm{R}=1.5 \mathrm{~mm}$ will be used as input dimensions for the modeling of the weld bead of the junction. The resulting geometry of the junction models are shown in Figure 79. The corner of the junctions has been modeled as an independent body in order to apply concrete mesh refinement conditions as will be shown later.

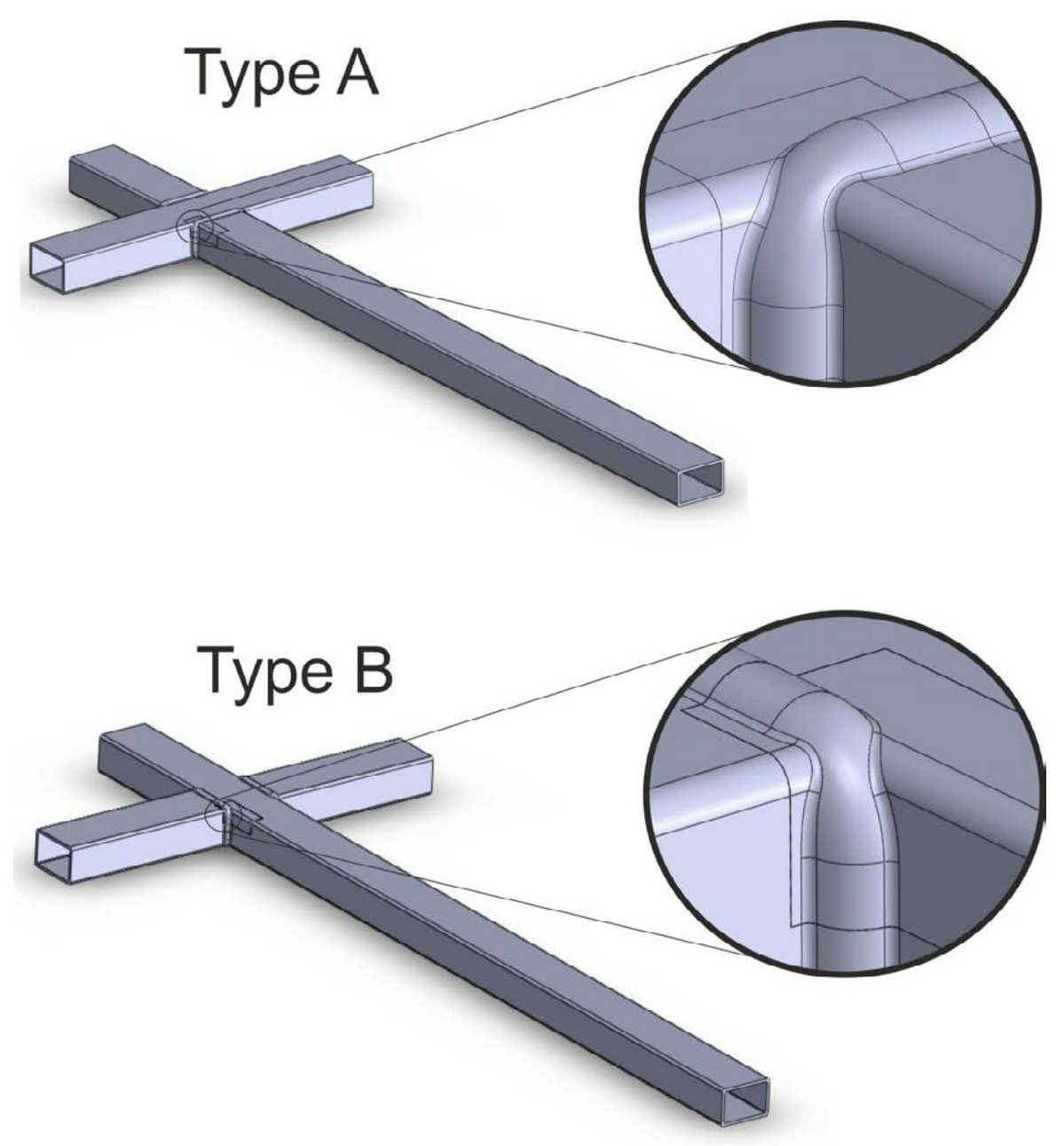

Figure 79 Junction models.

\subsubsection{MATERIAL}

Although no significant plastification was observed in the tested junctions in the range of the applied loads, most of the peak strain values obtained exceed the proportionality limit. Despite the behavior of the material is still considered elastic up to the yield point, the young modulus start to decrease from this point.

In order to avoid any source of deviation arising from local overstepping of the proportionality limit, it was decided to consider the material as nonlinear, and adjust it to the flow curve determined experimentally in Section 5.1 by means of the multilinear approximation shown in Figure 80. It was decided to use such multilinear material models instead of a constitute model due to the higher flexibility that it shows to make iterative adjustments. 


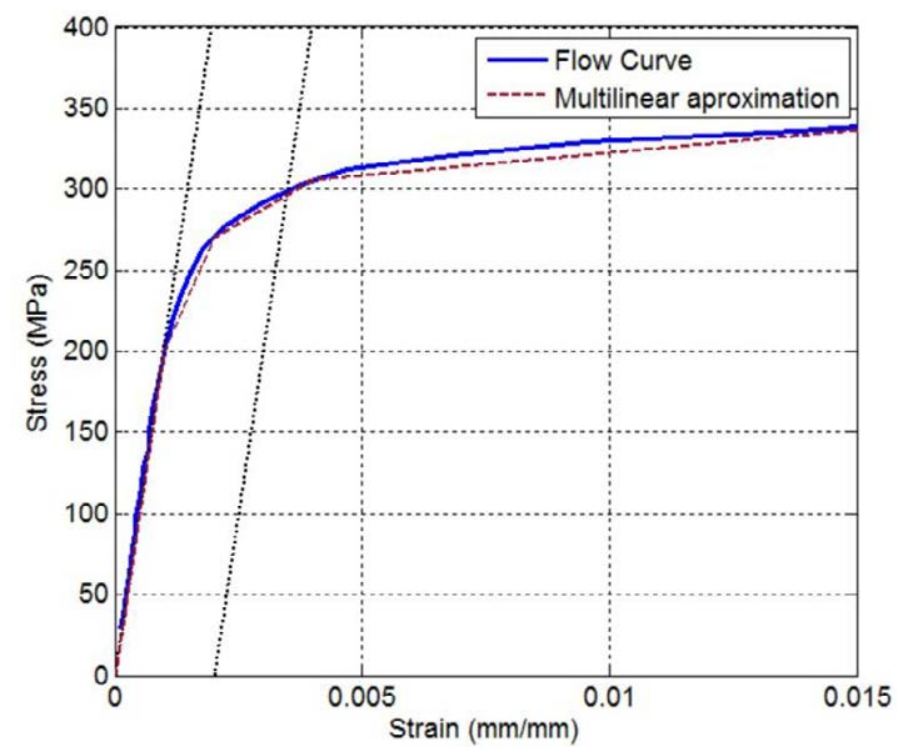

Figure 80 Multilinear approximation to flow curve.

It should be pointed that the base material will likely follow a slightly different flow curve from the one of the weld toe. Nevertheless, the elastic modulus of a structural steel such as the base material (S275J) rounds 200GPa. Additionally, non-linear behavior will not take place in this base material for the range of the applied loads. In this scenario, no significant deviation is expected to be introduced on the calculation by using the same material properties on the entire model.

\subsubsection{MESHING CHARACTERISTICS}

An appropriate meshing process is crucial for obtaining reliable results. Those zones of the model where the strain gradient is small can be accurately calculated using a coarse meshing of linear elements. On the contrary, if precise outcomes are necessary in areas where the strain field is highly heterogeneous (i.e. strain concentration areas), utilizing a sufficiently small element size becomes compulsory. The International Institute of Welding (IIW) (W. Fricke, 2010) states that the mesh size on a weld section should be small enough to guarantee the existence of at least three elements along the weld bead toe in case of using second order elements. Following this recommendation, the studied junctions should have an approximate element size of $0.28 \mathrm{~mm}$ in this area and quadratic elements type should be employed.

Local mesh refinement in the vicinity of the weld bead corner was performed in order to ensure meeting the IIW recommendation. From this zone, the element size was gradually increased up to the nominal value of $5 \mathrm{~mm}$ used for the rest of the model. In all, the meshes of Type A and Type B junctions are composed respectively of 69800 and 66300 quadratic hexahedral elements. The meshed models are shown in Figure 81, where it can be noted that the length of the fixed support have been reduced in order to optimize calculation process, since these zones will not have any contribution to the response of the junction. Similarly, the length of the longest arm of the junction was set to $840 \mathrm{~mm}$, corresponding to the point of load application, the extra length of the was also removed for calculation process optimization. Additionally, the corner where the mesh refinement was performed was modeled as a different 
body in order to apply independent mesh conditions. Both bodies are joined by means of 'bonded' contact conditions which ensures no relative displacement in the contact faces.
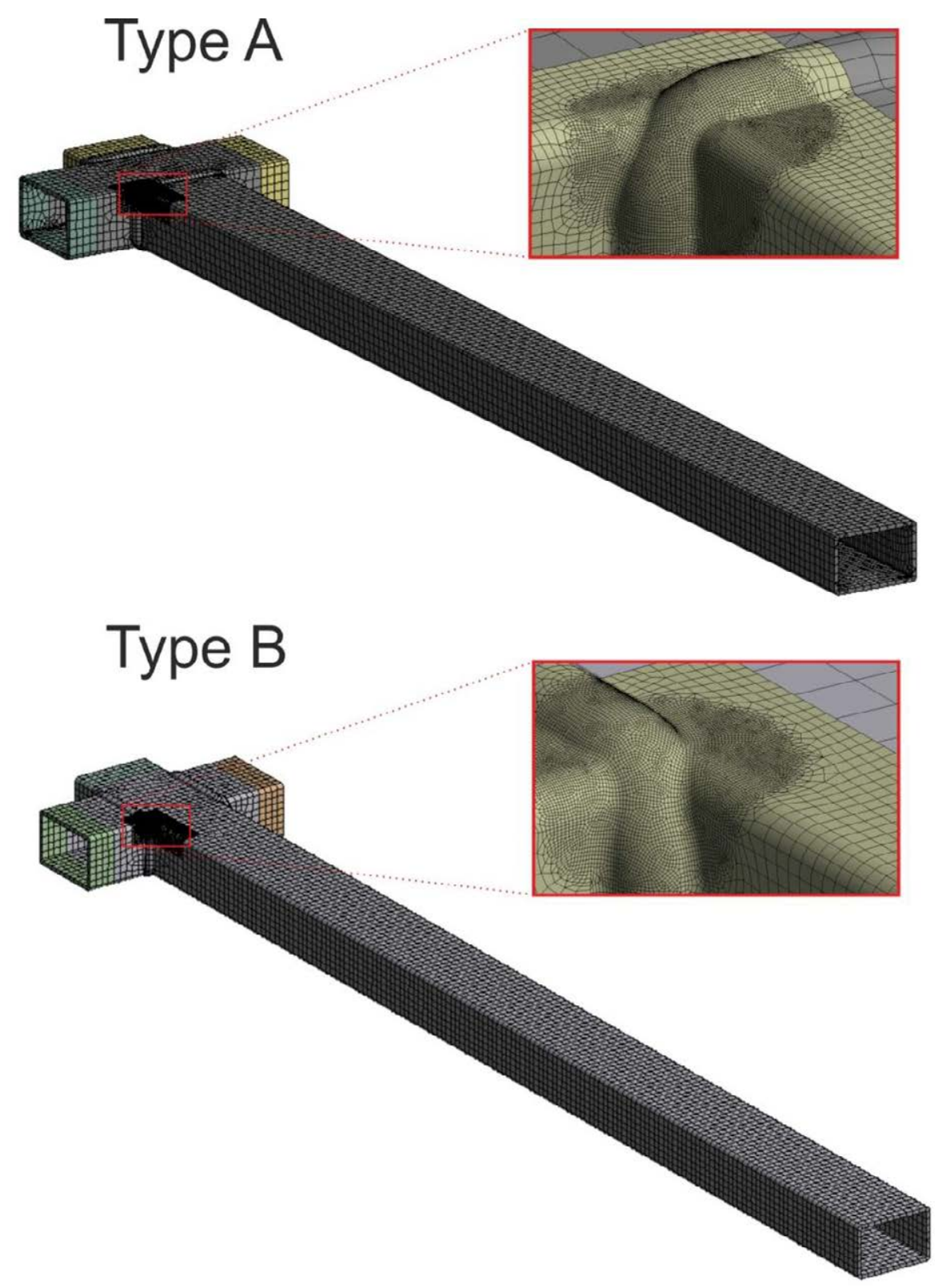

Figure 81 Meshed junction models

Finally, it should be pointed that a complete LPTV model constructed with such detailed junctions, given that they are formed approximately by 520 welded junctions (F. Badea, 2014), would suppose to employ around $35 \mathrm{e} 6$ quadratic elements. It is easy to deduce that running the load cases extracted from the Rainflow data over such heavy model supposes a non feasible option.

\subsubsection{BOUNDARY CONDITIONS}

With respect to the displacement constrains, fixed supports were set at the areas of the junctions corresponding with those clamped in the actual tests (Figure 60). 
Regarding load conditions, in order to assure equivalent load states both in experimental test and virtual calculation, the input of the later should be the forces obtained for the different imposed displacements. In this manner, any deviation arising from the stiffness difference between the clamping of the actual junction and the fixed support of the virtual model of the junctions is overcame.

It should be kept in mind that displacement restrictions in FEM models is equivalent to an infinitely stiff clamping system, which is impossible to achieve in actual tests. Therefore, higher forces would be needed to meet the same displacement conditions, leading to higher strains.

On the contrary, given that the stiffness of the welded junction is very similar in both actual junctions and virtual models since geometry has been carefully modeled, the load state will in turn be very similar, and the total deflection of the FEM model will be less due to the mentioned higher stiffness of the fixed supports.

This fact is easily observed by the relation of the nominal strain on the vicinity the corner, which writes as follows according to the classical strength of materials formulation.

$$
\varepsilon=\frac{\mathrm{M} c}{E I}=\frac{(F d) \mathrm{c}}{E I}
$$

Where $F$ is the applied force, $d$ is the distance from the junction corner to the point of load application, $c$ is the distance from the cross section center to the top face of the profile, $E$ is the young modulus and $I$ is the moment of inertia of the tube beam cross section. It can be seen in this relation how the nominal bending strain will increase linearly as the applied force increases.

For validation purposes, the corresponding loads to imposed displacements of $+12.5 \mathrm{~mm}$ and $+7.5 \mathrm{~mm}$ for the $Z$ and $Y$ load directions respectively were used. The following Table 22 and

Table 23 gather the force values recorded for these displacements over the different junctions tested. The average force values obtained for each junction type and load direction will be used as the input forces on the FEM models. The forces will be applied to the end face of the long profile of the models shown in Figure 81.

Table 22: Force needed to impose displacements of $+\mathbf{1 2 . 5} \mathrm{mm}$ and $+7.5 \mathrm{~mm}$ for $\mathrm{Z}$ and $\mathrm{Y}$ directions respectively. For 'Type $A$ ' junctions.

\begin{tabular}{|c|c|c|}
\hline Junction no $^{\mathbf{0}}$ & Z Force (N) $^{\text {Y Force (N) }}$ \\
\hline 1 & 1277 & 1536 \\
\hline 2 & 1298 & 1593 \\
\hline 3 & 1288 & 1598 \\
\hline 4 & 1307 & 1547 \\
\hline 5 & 1276 & 1684 \\
\hline Mean & $\mathbf{1 2 8 9}$ & $\mathbf{1 5 9 2}$ \\
\hline
\end{tabular}


Table 23: Force needed to impose displacements of $12.5 \mathrm{~mm}$ and $7.5 \mathrm{~mm}$ for $Z$ and $Y$ directions respectively. For 'Type B' junctions.

\begin{tabular}{|c|c|c|}
\hline Junction n $^{\mathbf{0}}$ & Z Force (N) & Y Force (N) \\
\hline 1 & 1432 & 1665 \\
\hline 2 & 1425 & 1676 \\
\hline 3 & 1450 & 1730 \\
\hline 4 & 1423 & 1550 \\
\hline 5 & 1440 & 1670 \\
\hline Mean & $\mathbf{1 4 3 4}$ & $\mathbf{1 6 5 9}$ \\
\hline
\end{tabular}

\subsection{FINITE ELEMENT MODEL VALIDATION}

In total, 4 different FEM results need to be validated against experimental data, coming from two load directions for each of the two junctions types evaluated. The solutions settings of the models are summarized in Table 24 . The total time employed to solve each model rounds the 5 minutes in 6 core T1100 AMP processor with 16Gb of RAM memory.

Table 24: Solution information.

\begin{tabular}{|c|c|}
\hline Number of steps & 1 \\
\hline Initial number of substeps & 1 \\
\hline Minimum number of substeps & 1 \\
\hline Maximum number of substeps & 10 \\
\hline Auto time stepping & On \\
\hline Solver & Direct with sparse matrix \\
\hline Solution controls & Full Newton-Raphson \\
\hline Convergence controls & Use defaults \\
\hline
\end{tabular}

The following Figure 82 shows the strain fields obtained for each case in the vicinity of the junction corner. Good agreement is observed from a qualitative comparison with Figure 66, which shows the equivalent strain fields obtained experimentally. 

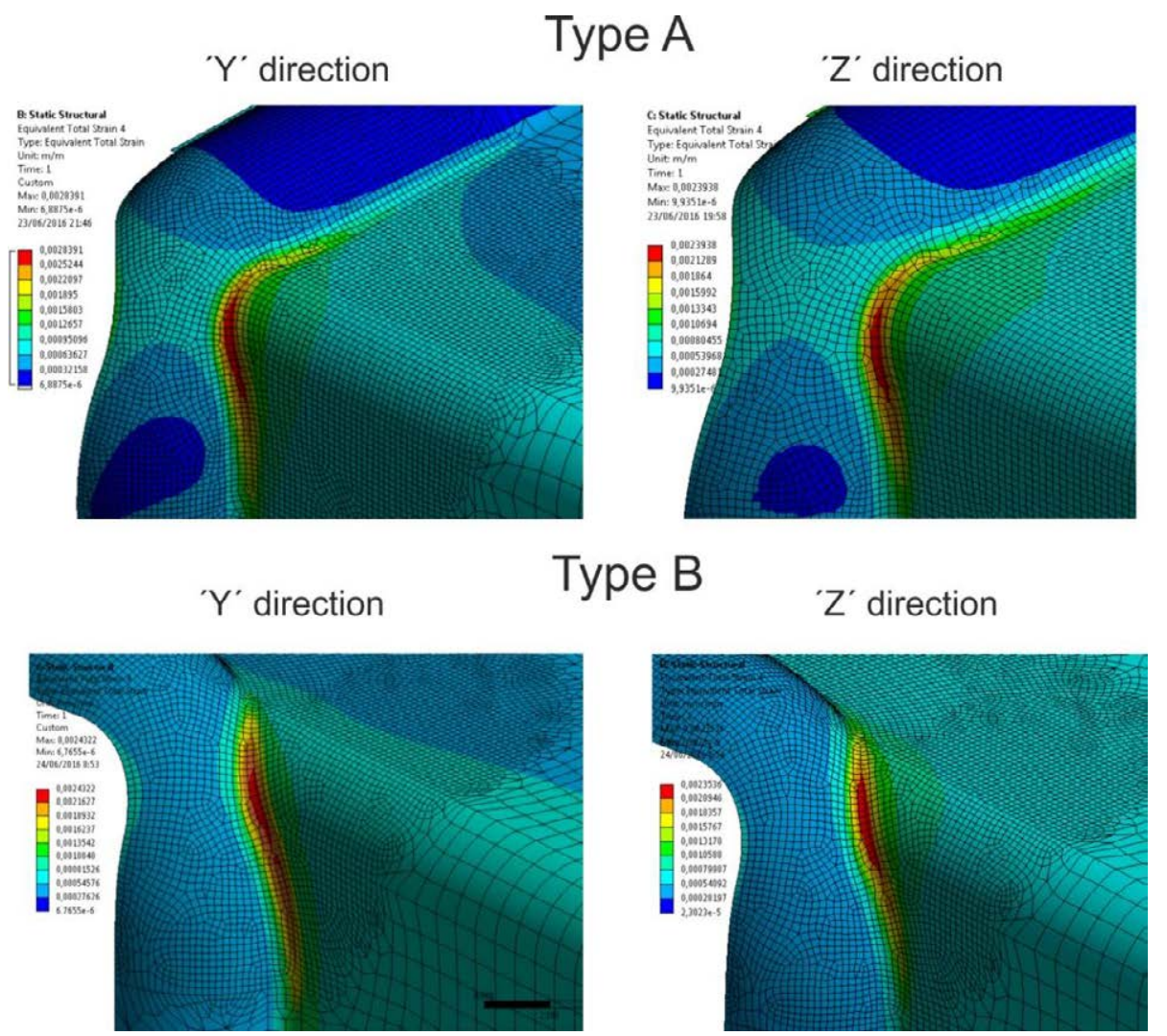

Figure 82 Strain fields obtained from the FEM models

Additionally, in order to assess the consistency of the nominal strain theoretical approach, the strain outputs over a path that moves in $\mathrm{X}$ direction from the maximum value obtained were extracted and compared to the ones obtained theoretically. This comparative is shown in Figure 83. Good agreement between FEM results and theoretical calculation is found. It can be seen on the graphs that the strain take between 8 to $13 \mathrm{~mm}$ to meet the nominal values. This distance is very close to the limit of regions of interest on DIC measurements, which explains the difficulties found when trying to obtain nominal strain values from DIC data extrapolation.
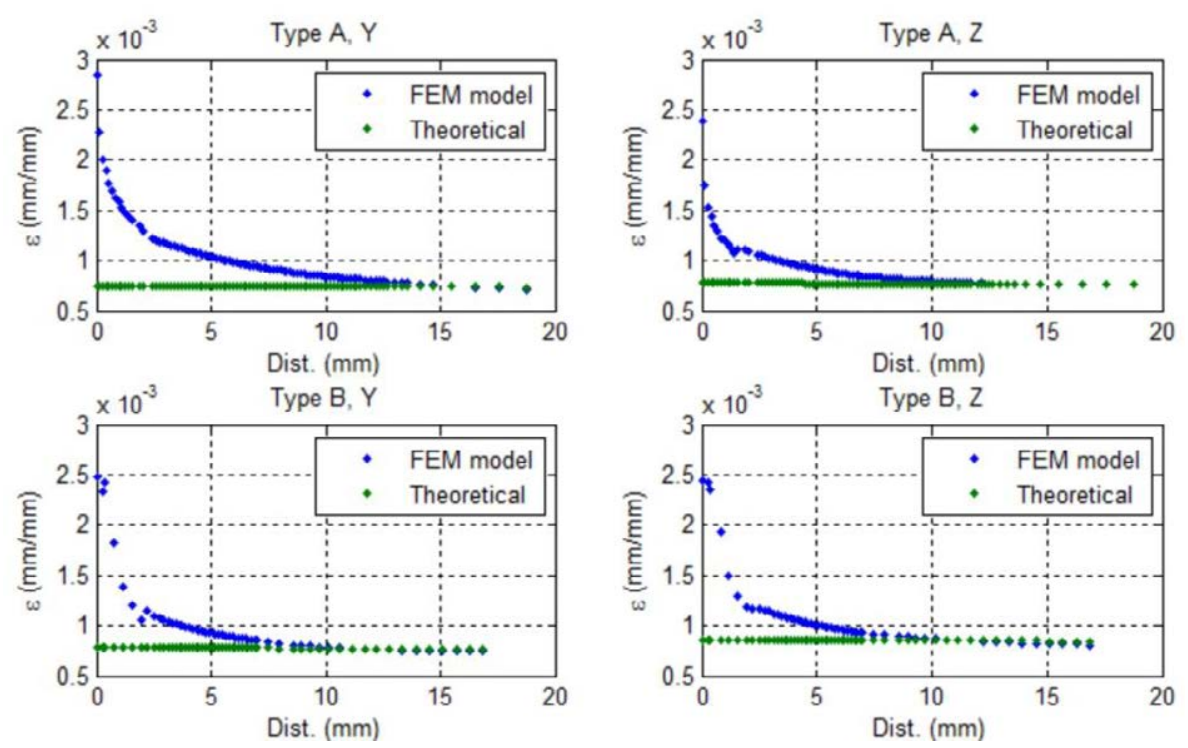

Figure 83 Strain evolution: FEM models versus theoretical nominal 
Although the similarities between tested and calculated strain fields might be a good indicator of the accurate behavior of the later, in fatigue calculations is imperative a precise agreement between peak strain values, or in turn in the SCF, since it will mark the highest strain cycle and therefore the least fatigue life point. The following Table 25 shows the peak strains and SCF values obtained from the FEM models and the Figure 84 shows graphically the SCF values over the experimental observation. It can be observed that the scatter of the SCF phenomenon itself is already significantly larger than the deviation of the FEM outcomes.

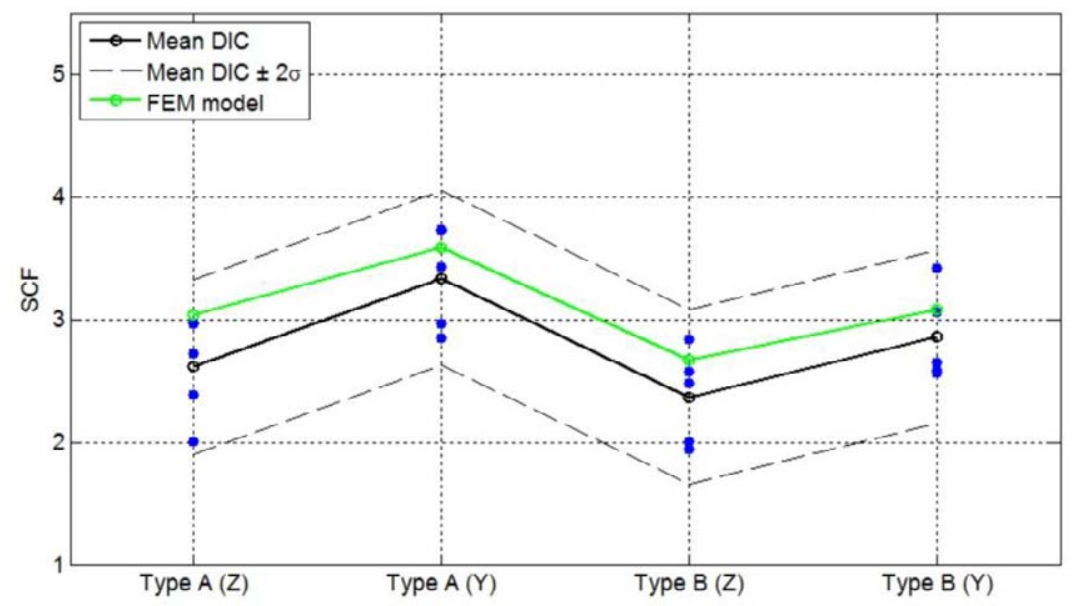

Figure 84 SCF comparison between DIC observation and FEM model results

Table 25: Strain and SCF values obtained from FEM model results.

\begin{tabular}{|c|c|c|c|}
\hline Junction -Load & $\boldsymbol{\varepsilon}_{\max }$ & $\boldsymbol{\varepsilon}_{\text {nom }}$ & SCF \\
\hline Type A - Z direction & 0.00239 & $7.77 \mathrm{e}^{-4}$ & 3.07 \\
\hline Type A - Y direction & 0.00284 & $7.75 \mathrm{e}_{-4}$ & 3.66 \\
\hline Type B - Z direction & 0.00235 & $8.62 \mathrm{e}_{-4}$ & 2.73 \\
\hline Type B - Y direction & 0.00243 & $7.81 \mathrm{e}_{-4}$ & 3.11 \\
\hline
\end{tabular}

In order to quantify the deviation of the FEM model outcomes, a validation metric (W.L. Oberkampf et al., 2006) based in confidence intervals was employed. In accordance to the central limit theorem, the probability interval with a level of confidence $100(1-\alpha) \%$ for the true SCF mean $(\mu)$ for each junction type (i) and load direction (j) can be denoted as:

$$
\mu_{(i, j)} \sim\left(\bar{K}_{e(i, j)}-t_{\alpha / 2, v} \cdot \frac{\sigma_{S C F}}{\sqrt{n}}, \bar{K}_{e(i, j)}+t_{\alpha / 2, v} \cdot \frac{\sigma_{S C F}}{\sqrt{n}}\right)
$$

Where $\sigma_{S C F}$ is the standard deviation of the SCF experimental observations determined in Section 5.2, $n$ is the number of observations ( 5 in this case: for each load level and junction type) and ' $t_{\alpha / 2, v}$ ' is the T-student distribution with $v$ degrees of freedom ( $n-1$ observations). The following Figure 85 shows these intervals for a level of confidence of $95 \%$ plotted over the FEM results for each junction and load type. 


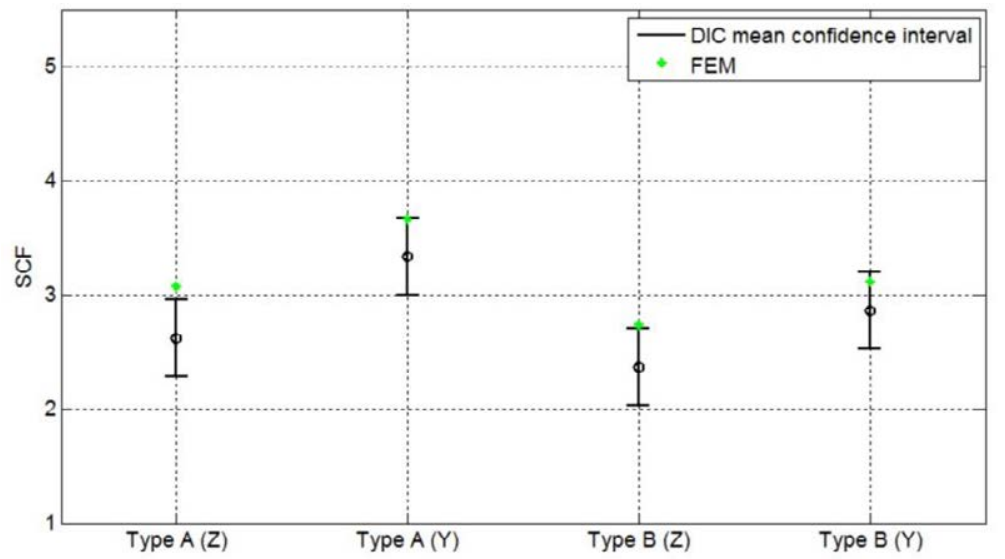

Figure 85 Mean SCF confidence intervals and FEM model results

Additionally, it is known that the error is defined as the deviation between the actual and predicted values, in this case:

$$
E_{(i, j)}=K_{F E M(i, j)}-\mu_{(i, j)}
$$

Where $K_{F E M(i, j)}$ is the predicted SCF by the FEM model of each jucntion and load type. The error can be also expressed in relative terms as:

$$
E_{\text {rel(i,j) }}(\%)=\frac{K_{F E M(i, j)}-\mu_{(i, j)}}{\mu_{(i, j)}} \cdot 100
$$

Finally, the corresponding interval of the true absolute error can be obtained by substituting eq.(1.108) into eq.(1.107):

$$
E_{(i, j)} \sim\left(\left(\bar{K}_{e(i, j)}-K_{F E M(i, j)}\right)-t_{\alpha / 2, v} \cdot \frac{\sigma_{S C F}}{\sqrt{n}},\left(\bar{K}_{e(i, j)}-K_{F E M(i, j)}\right)+t_{\alpha / 2, v} \cdot \frac{\sigma_{S C F}}{\sqrt{n}}\right)
$$

Similarly, by substituting eq.(1.109) into eq.(1.107), the interval of the relative error is written as:

$$
E_{r e l(i, j)} \sim\left(\frac{K_{F E M(i, j)}}{\bar{K}_{e(i, j)}-t_{\alpha / 2, v} \cdot \frac{\sigma_{S C F}}{\sqrt{n}}}-1, \frac{K_{F E M(i, j)}}{\bar{K}_{e(i, j)}+t_{\alpha / 2, v} \cdot \frac{\sigma_{S C F}}{\sqrt{n}}}-1\right) \cdot 100
$$

The limits of the absolute and relative error intervals for a confidence level of $95 \%$ are shown in Figure 86 and Figure 87 respectively. A general good agreement is seen between FEM calculations and DIC measurements, specially for loads in ' $Y$ ' direction, were the average relative error is of $9.6 \%$ and $8.7 \%$ percent for Type 'A' and Type 'B' junctions respectively.

It is also noticed that in all cases the error is positive, meaning that the predicted SCF is higher than the actual one, which will lead to slightly conservative results. This tendency is more accentuated for loads in ' $Z$ ' direction, were the average relative error scales to $17.4 \%$ and $15.1 \%$ 
percent for Type ' $A$ ' and Type ' $B$ ' junctions respectively. In any case, these deviations are notably small in comparison with the proper scatter of the SCF itself as was observed in Figure 84.

Finally, it is seen that the FEM models behave in accordance with experimental observations in terms of strain concentration behavior tendency, as in both cases the configuration with the highest SCF is junction Type ' $A$ ' and ' $Y$ ' load direction, followed by junction Type ' $B$ ' and ' $Y$ ' load direction, junction Type ' $A$ ' and ' $Z$ ' load direction, and junction Type 'B' and ' $Z$ ' load direction.

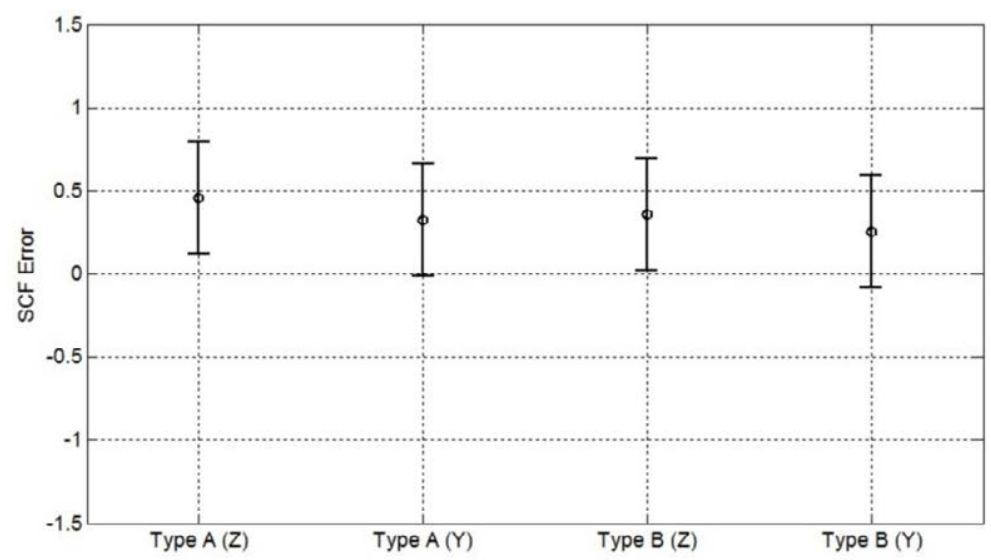

Figure 86 FEM model error intervals

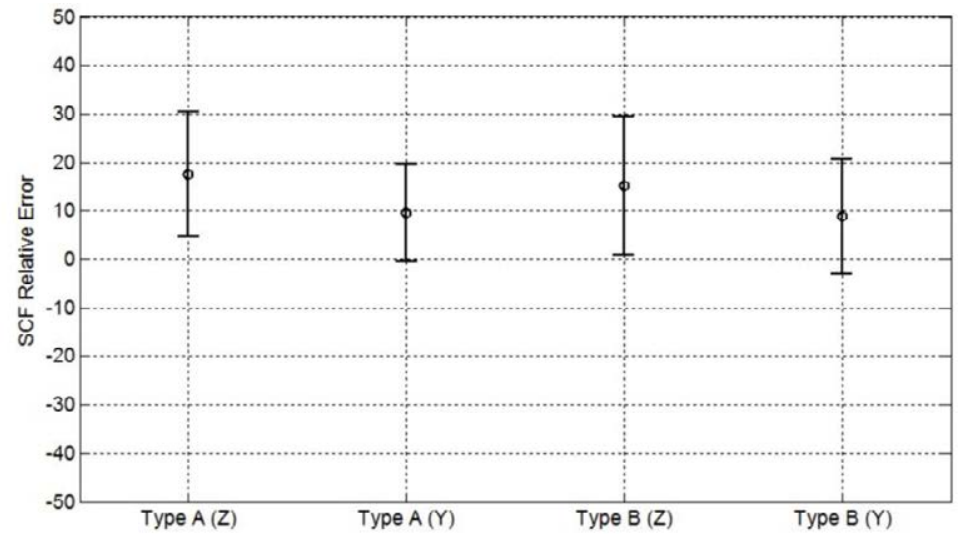

Figure 87 FEM model relative error intervals

As a result, the global behavior of the virtual models constructed on the basis of the technique described in this section is considered appropriated for the study of present thesis. Showing slightly conservative results, but good agreement in terms of values and tendency. This modeling procedure will be therefore employed to construct the detailed junction models of the subsequent sections, that will be used to establish a relation between them and simplified models built on the basis of beam type elements.

\subsection{STRAIN STATE UNDER COMBINED LOADS: PROPORTIONALITY STUDY}

Once the model has been validated to provide accurate results, and additional evaluation that should be carried out is the evolution strain state of junction under combined loads, that is, 
when applied loads in both ' $Z$ ' and ' $Y$ ' directions simultaneously. This situation will actually be the most frequent un actual junctions old LPTV structures. The objective is to assess whether the strain state shows proportionality under different load combinations. As was already stated in Section 3.1., fatigue approaches might vary significantly from proportional to nonproportional loading cases.

To do so, both type ' $A$ ' and type 'B' models were first calculated for the load steps described in Figure 88. The main idea is to cover a wide variety of possible combinations of loads in ' $Y$ ' and ' $Z$ ' directions. Low load level were chosen in order to avoid possible results biasing due to local material plastification. The rest of the boundary conditions and settings were left unchanged.

Tabular Data
\begin{tabular}{|l|l|l|l|l|l|l|}
\hline & Steps & Time [s] & $\sqrt{ } X[\mathrm{~N}]$ & $\sqrt{ }$ \\
\hline 1 & 1 & 0, & $=0$, & 100, & $=0$, \\
\hline 2 & 1 & 0,1 & $=0$, & 100, & 0, \\
\hline 3 & 2 & 0,2 & $=0$, & 100, & 25, \\
\hline 4 & 3 & 0,3 & $=0$, & 100, & 50, \\
\hline 5 & 4 & 0,4 & $=0$, & 100, & 75, \\
\hline 6 & 5 & 0,5 & $=0$, & 100, & 100, \\
\hline 7 & 6 & 0,6 & $=0$, & 75, & 100, \\
\hline 8 & 7 & 0,7 & $=0$, & 50, & 100, \\
\hline 9 & 8 & 0,8 & $=0$, & 25, & 100, \\
\hline 10 & 9 & 0,9 & 0, & 0, & 100, \\
\hline
\end{tabular}

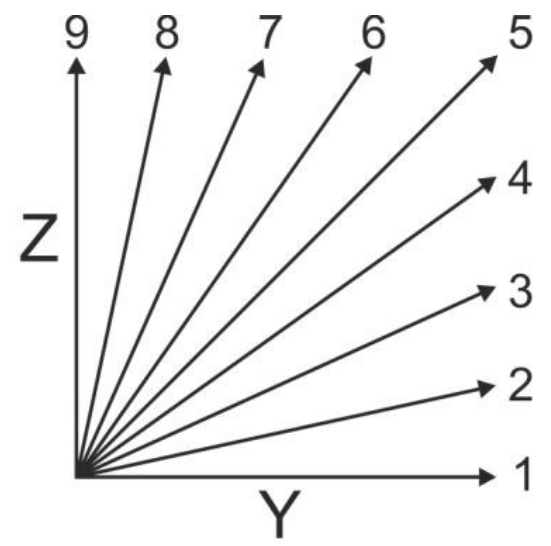

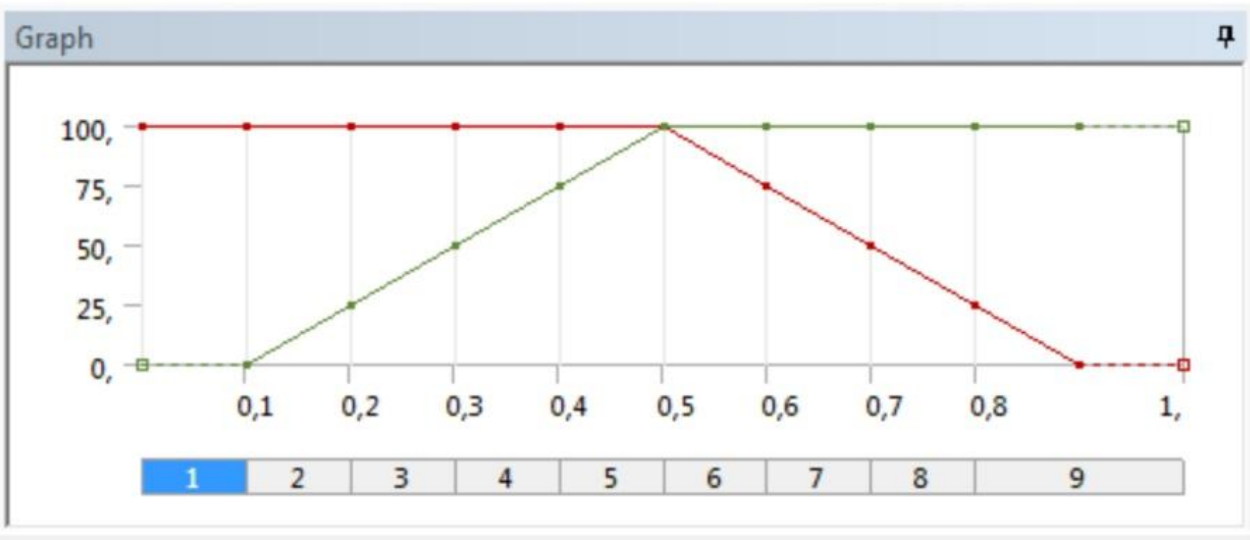

Figure 88 Proportionality study load cases

Afterwards, it was selected a node situated in the vicinity of the highest strains to monitor the evolution of its principal components. The following Table 26 and

Table 27 shows these outcomes as well as the equivalent strain for Type ' $A$ ' and Type 'B' junction types respectively.

From the observation of the results tables, it can be easily deduced that the equivalent Von Mises strain of a combined load state is equivalent to the sum of the equivalent strains of its components, in agreement with the superposition principle. 
Table 26: Strain outcomes for Type 'A' junction model.

\begin{tabular}{|c|c|c|c|c|c|c|}
\hline $\begin{array}{c}\text { Load } \\
\text { Step }\end{array}$ & $\begin{array}{c}\text { Z Force } \\
(\mathbf{N})\end{array}$ & $\begin{array}{c}\text { Y Force } \\
(\mathbf{N})\end{array}$ & $\boldsymbol{\varepsilon}_{\mathbf{1}}$ & $\boldsymbol{\varepsilon}_{\mathbf{2}}$ & $\boldsymbol{\varepsilon}_{\mathbf{3}}$ & $\boldsymbol{\varepsilon}_{\mathbf{V M}}$ \\
\hline 1 & 0 & 100 & $1.9 \mathrm{e}^{-4}$ & $-6.77 \mathrm{e}^{-5}$ & $-2.25 \mathrm{e}^{-5}$ & $1.88 \mathrm{e}^{-4}$ \\
\hline 2 & 25 & 100 & $2.33 \mathrm{e}^{-4}$ & $-8.32 \mathrm{e}^{-5}$ & $-2.75 \mathrm{e}^{-5}$ & $2.30 \mathrm{e}^{-4}$ \\
\hline 3 & 50 & 100 & $2.76 \mathrm{e}^{-4}$ & $-9.87 \mathrm{e}^{-5}$ & $-3.31 \mathrm{e}^{-5}$ & $2.72 \mathrm{e}^{-4}$ \\
\hline 4 & 75 & 100 & $3.18 \mathrm{e}^{-4}$ & $-1.14 \mathrm{e}^{-4}$ & $-3.86 \mathrm{e}^{-5}$ & $3.15 \mathrm{e}^{-4}$ \\
\hline 5 & 100 & 100 & $3.61 \mathrm{e}^{-4}$ & $-1.30 \mathrm{e}^{-4}$ & $-4.34 \mathrm{e}^{-5}$ & $3.57 \mathrm{e}^{-4}$ \\
\hline 6 & 100 & 75 & $3.14 \mathrm{e}^{-4}$ & $-1.13 \mathrm{e}^{-4}$ & $-3.74 \mathrm{e}^{-5}$ & $3.10 \mathrm{e}^{-4}$ \\
\hline 7 & 100 & 50 & $2.66 \mathrm{e}^{-4}$ & $-9.59 \mathrm{e}^{-5}$ & $-3.13 \mathrm{e}^{-5}$ & $2.63 \mathrm{e}^{-4}$ \\
\hline 8 & 100 & 25 & $2.19 \mathrm{e}^{-4}$ & $-7.90 \mathrm{e}^{-5}$ & $-2.53 \mathrm{e}^{-5}$ & $2.16 \mathrm{e}^{-4}$ \\
\hline 9 & 100 & 0 & $1.71 \mathrm{e}^{-4}$ & $-6.21 \mathrm{e}^{-5}$ & $-1.93 \mathrm{e}^{-5}$ & $1.69 \mathrm{e}^{-4}$ \\
\hline
\end{tabular}

Table 27: Strain outcomes for Type 'B' junction model.

\begin{tabular}{|c|c|c|c|c|c|c|}
\hline $\begin{array}{c}\text { Load } \\
\text { Step }\end{array}$ & $\begin{array}{c}\text { Z Force } \\
(\mathbf{N})\end{array}$ & $\begin{array}{c}\text { Y Force } \\
(\mathbf{N})\end{array}$ & $\boldsymbol{\varepsilon}_{\mathbf{1}}$ & $\boldsymbol{\varepsilon}_{\mathbf{2}}$ & $\boldsymbol{\varepsilon}_{\mathbf{3}}$ & $\boldsymbol{\varepsilon}_{\mathbf{V M}}$ \\
\hline 1 & 0 & 100 & $1.36 \mathrm{e}^{-4}$ & $-4.94 \mathrm{e}^{-5}$ & $-2.01 \mathrm{e}^{-5}$ & $1.33 \mathrm{e}^{-4}$ \\
\hline 2 & 25 & 100 & $1.77 \mathrm{e}^{-4}$ & $-6.43 \mathrm{e}^{-5}$ & $-2.56 \mathrm{e}^{-5}$ & $1.73 \mathrm{e}^{-4}$ \\
\hline 3 & 50 & 100 & $2.18 \mathrm{e}^{-4}$ & $-7.93 \mathrm{e}^{-5}$ & $-3.11 \mathrm{e}^{-5}$ & $2.12 \mathrm{e}^{-4}$ \\
\hline 4 & 75 & 100 & $2.58 \mathrm{e}^{-4}$ & $-9.42 \mathrm{e}^{-5}$ & $-3.66 \mathrm{e}^{-5}$ & $2.52 \mathrm{e}^{-4}$ \\
\hline 5 & 100 & 100 & $2.99 \mathrm{e}^{-4}$ & $-1.09 \mathrm{e}^{-4}$ & $-4.21 \mathrm{e}^{-5}$ & $2.92 \mathrm{e}^{-4}$ \\
\hline 6 & 100 & 75 & $2.65 \mathrm{e}^{-4}$ & $-9.68 \mathrm{e}^{-5}$ & $-3.71 \mathrm{e}^{-5}$ & $2.58 \mathrm{e}^{-4}$ \\
\hline 7 & 100 & 50 & $2.31 \mathrm{e}^{-4}$ & $-8.45 \mathrm{e}^{-5}$ & $-3.25 \mathrm{e}^{-5}$ & $2.25 \mathrm{e}^{-4}$ \\
\hline 8 & 100 & 25 & $1.97 \mathrm{e}^{-4}$ & $-7.21 \mathrm{e}^{-5}$ & $-2.79 \mathrm{e}^{-5}$ & $1.92 \mathrm{e}^{-4}$ \\
\hline 9 & 100 & 0 & $1.63 \mathrm{e}^{-4}$ & $-5.98 \mathrm{e}^{-5}$ & $-2.29 \mathrm{e}^{-5}$ & $1.59 \mathrm{e}^{-4}$ \\
\hline
\end{tabular}

The following graphs show the relations between the three principal strains for Type ' $A$ ' and Type ' $B$ ' junction respectively. On the one hand, the nonzero values of all principal strains at the strain concentration zone, indicates the multiaxial strain state in this area. On the other hand, it is observed a linear relation among all the combinations of these strains, which is in accordance with the proportionality condition presented in eq.(1.32). Consequently, the fatigue strain cycles that the welded junctions will suffer under the different load combinations can be considered as proportional loads, and thus the corresponding multiaxial fatigue approach described in Section 3.1 can be employed. 

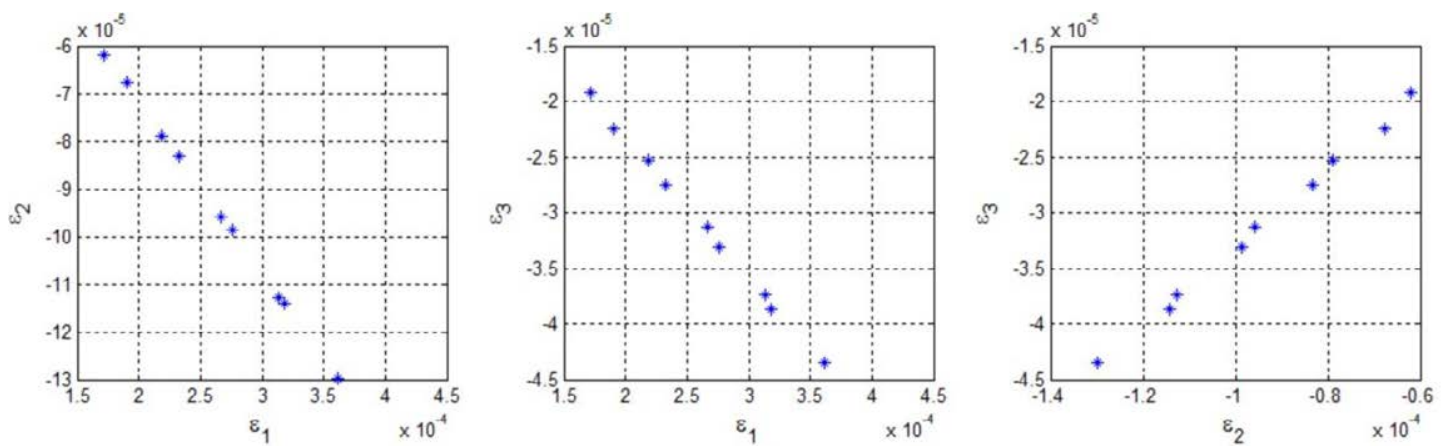

Figure 89 Principal strains relations for Type A junction.
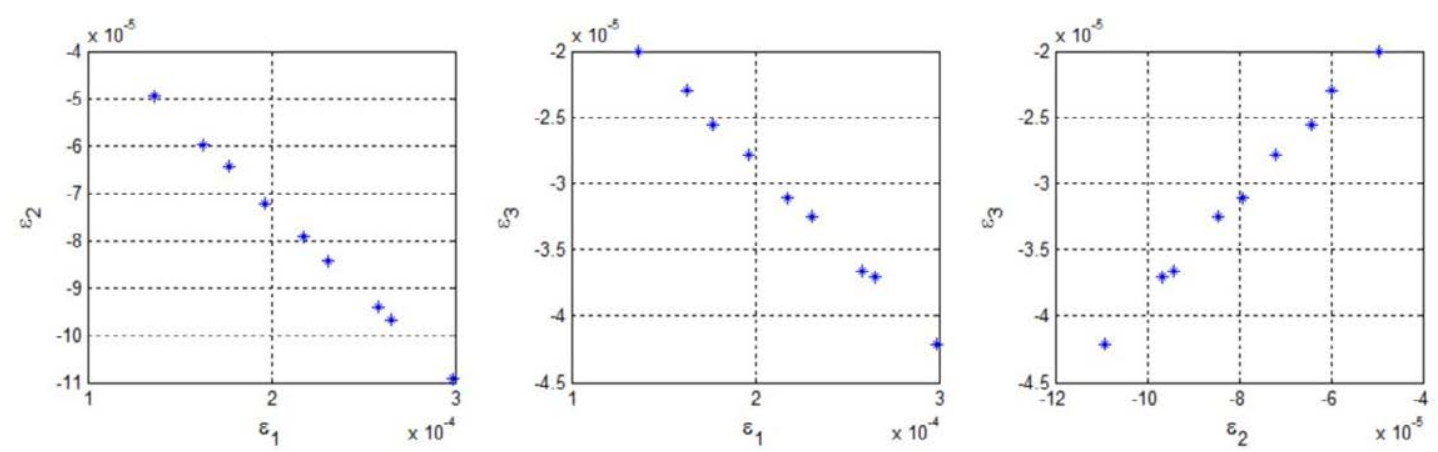

Figure 90 Principal strains relations for Type B junction. 


\section{CORRELATION BETWEEN DETAILED AND SIMPLIFIED MODELS OF RECTANGULAR TUBE BEAM WELDED JUNCTIONS}

As it was stated, LPTV structure models based on simplified beam type models cannot account strain concentrations since these type of elements are unable to adapt to the local stiffness changes provoked by the weld bead. Consequently, important deviations will be incurred by any fatigue approach based on the outcomes of these models.

This limitation could be overcame by using LPTV models that account for each junction geometrical details in an equivalent manner as the model validated in the previous section. Nevertheless, it would lead to a very complex model, consisting of millions of degrees of freedom that would suppose an enormous computational cost.

This section aims therefore to establish a series of correcting parameters that compensate the deviations of the simplified outcomes derived of these limitations. To do that, over 2000 welded junctions using different rectangular tube beam dimensions were calculated using both detailed and simplified modeling techniques. From the obtained results, regression models were constructed that permit establishing the aforementioned correcting factors for any dimensional combination of the profiles joined.

Finally, the regression models were validated by assessing its accuracy in new models with different dimensions from the ones used initially.

The subsequent sections describe this process in further detail.

\subsection{BEAM MODEL DESCRIPTION}

Modeling based on beam type elements are constructed using lines, since this type of elements are one-dimensional. The cross section characteristics are configured separately in order to provide the elements with the necessary information about the geometrical stiffness (i.e. inertial moments).

Using this modeling technique to construct welded junctions of tube beams lead to a configuration of four lines that converge to a single node representing the junction itself (Figure 91). A direct consequence of this modeling technique is the impossibility of making a difference between the type of junction: both Type ' $A$ ' and ' $B$ ' junction lead to the same simplified model. Additionally, a single node does not have the ability to deform, so the stiffness of the junction itself will be infinite (F. Badea et al. 2014). 


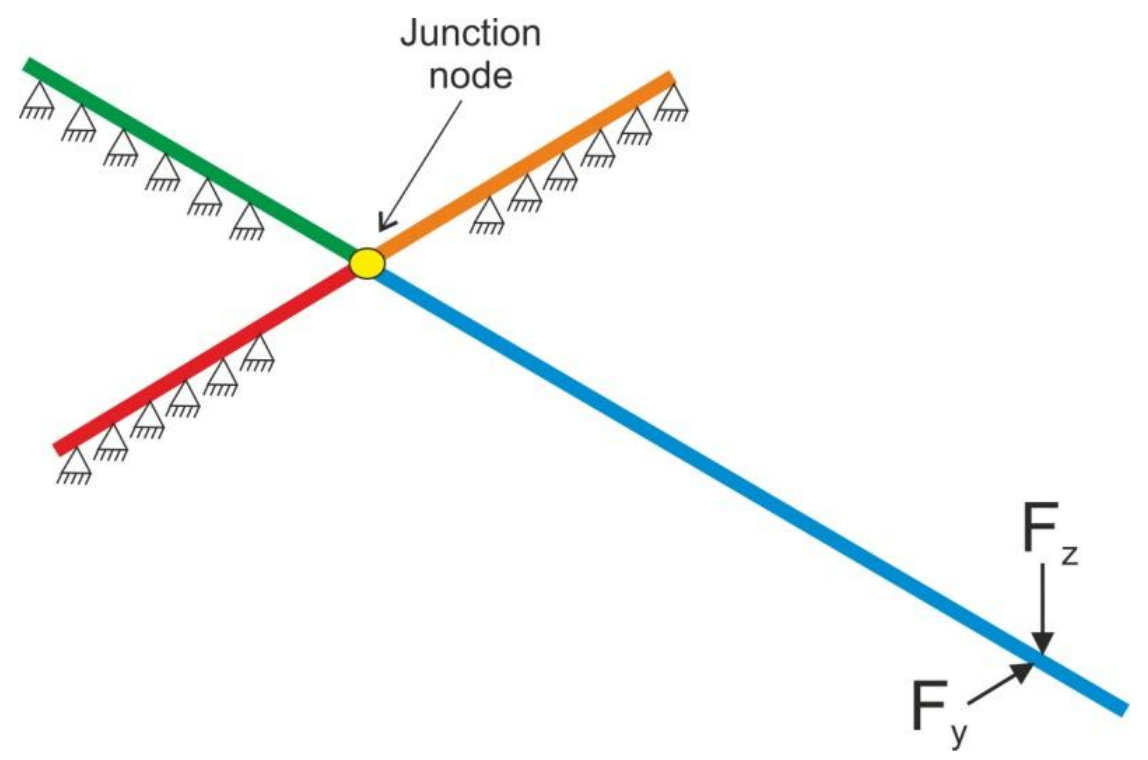

Figure 91 Simplified model of the welded junctions.

Like in the case of the detail models, the commercial software Ansys ${ }^{\circledR}$ was used to model and solve the simplified models of the junctions, and the element type BEAM188 was used. This type of element is based on Timoshenko beam theory, which considers constant shear strain over the section, i.e. cross sections remain plane after the deformation, although not perpendicular to the neutral axis of the beam. This type of beam formulation is preferred with respect to alternatives based on Euler-Bernoulli for modeling complex structures where some short beam might exist, since it shows significant precision improvement in these cases (A.T. Beck \& C.R.A. da Silva, 2011, Ansys ${ }^{\circledR}$ R14.0 Help).

The element BEAM188 is a two node element with 6 degrees of freedom in each node, including translations in the $x, y$, and $z$ directions and rotations about the $x, y$, and $z$ axis. $A$ third node can be optionally used to define the orientation of the beam. This element type can stand linear, quadratic or cubic shape functions. Higher order elements are recommended when the element might undergo highly heterogeneous deformations.

Quadratic, 3mm long elements were chosen to conform the mesh of the models of the present study. This configurations gives models that consists of 523 elements and 1047 nodes, of which half are mid-side internal nodes created to provide the element with quadratic shape function capacity. Finally, the total number of degrees of freedom of the model is 5322, slightly lower to the number of nodes times the DOF of each node $\left(1047^{*} 6\right)$, since some of them are fixed by the supports.

By way of example, the Von Mises strain results for loads of $100 \mathrm{~N}$ both ' $\mathrm{Z}$ ' and ' $\mathrm{Y}$ ' directions over a simplified welded junction model with the same dimensions as in the detailed models of previous Section 6 is shown in Figure 92. For a better understanding, the cross section of the beam elements has been rendered, nevertheless it is important to note that the geometry of these elements are one dimensional lines, and therefore the differences between Type ' $A$ ' and Type ' $B$ ' junctions cannot be modeled. 
It can be noticed that, as expected, no local strain raisings are accounted in these models, and in fact the maximum strains obtained in these models show great agreement with the nominal strains calculated by classic strength of material relation presented in eq. (1.106). Consequently, the relation between simplified and detailed models coincides with the SCF.
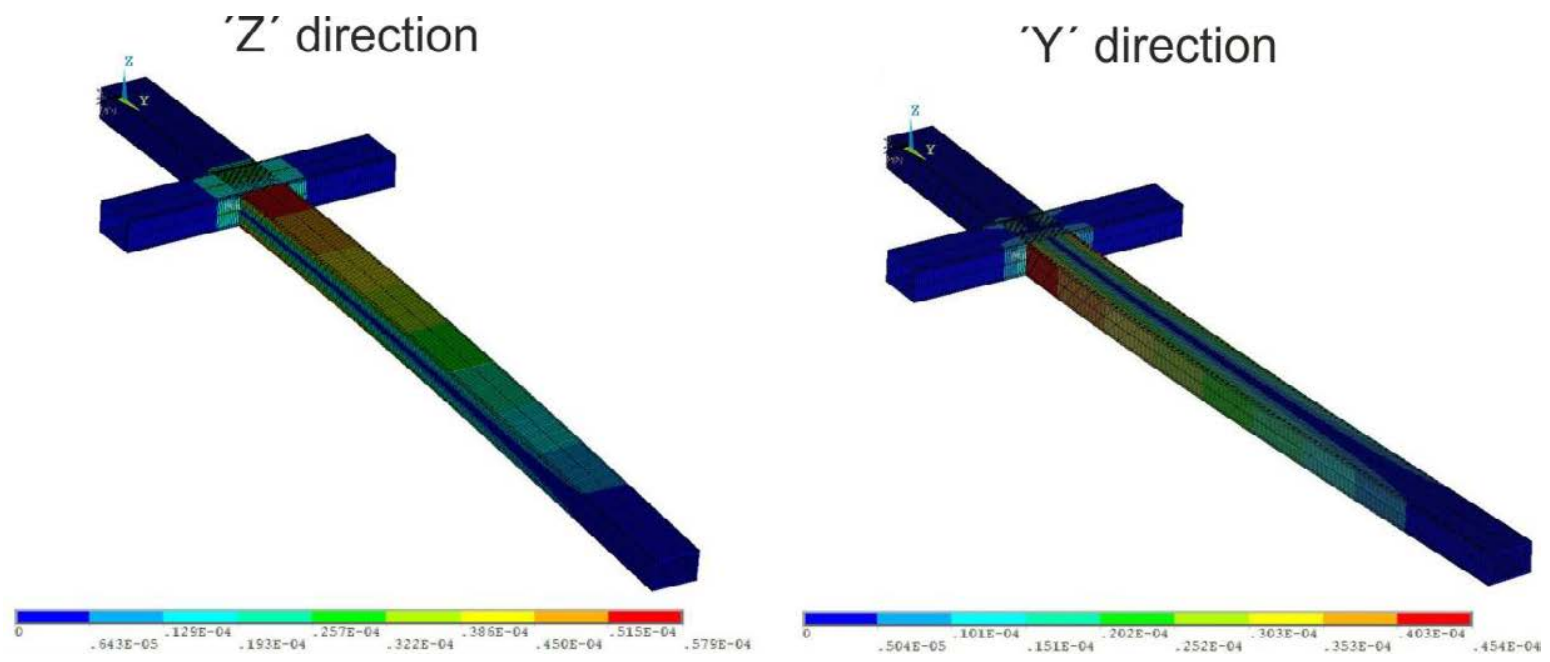

Figure 92 Simplified beam model Von Mises strain results.

Finally, it should be also pointed that coarser elements could be used in regions where the deformations are more homogeneous. Nevertheless, the total CPU time to solve these model is hardly a couple of seconds, so an extra operation of mesh refinement is not considered necessary in this case.

\subsection{RECTANGULAR TUBE BEAM DIMENSION COMBINATIONS CALCULATED}

A vast variety of combinations can be found in the dimensions of the tube beam welded junctions. In general terms, the cross section sides range between 20 and $80 \mathrm{~mm}$, and thicknesses between 2 and $4 \mathrm{~mm}$.

Additionally, a representative initial dimensional data from which to construct the regression models deviations is crucial for its accuracy. The possible dimensional variables that exist in a tube beam T-welded junction plus the two load directions are shown in the following Figure 93. 


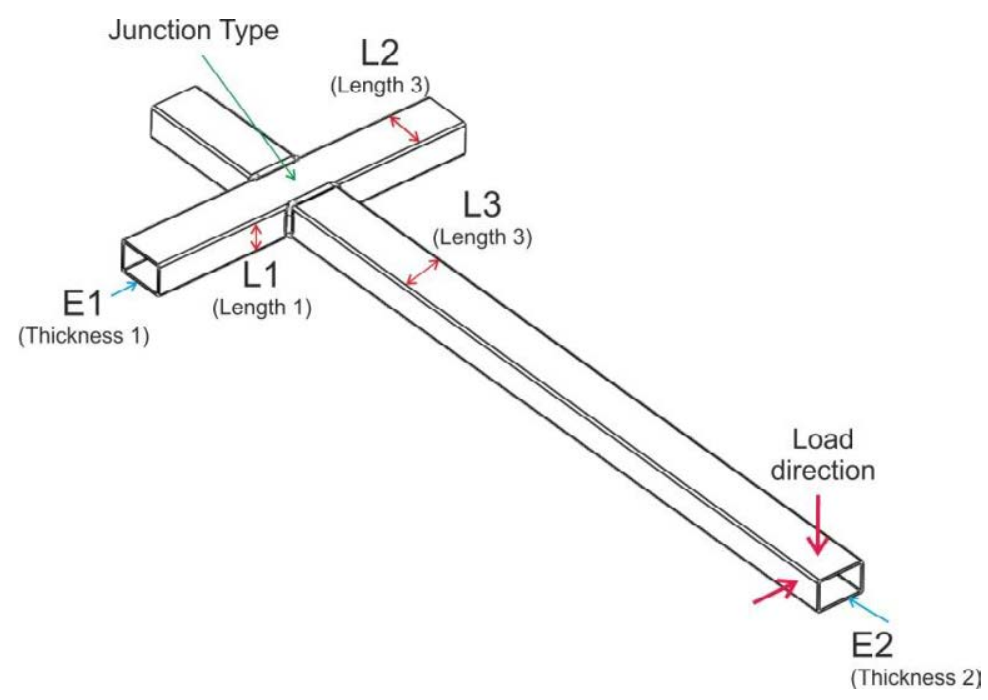

Figure 93 Dimensional variables of T-welded junctions.

The values of the different dimensional variables studied are summarized in Table 28. In total, there were constructed and calculated 2304 detailed and 1152 simplified models arising from all possible combinations of these values. It was developed a Matlab ${ }^{\circledR}$ script that modifies the models, calculates it and extracts maximum strains in an automated manner in order to save manipulation time. Additionally, a small force of $100 \mathrm{~N}$ was applied in both models and load directions in order to ensure linear behavior of all the models. Even so, there were necessary over 1000 computer hours to calculate all models. Obviously, the great majority of this computing cost arises from the calculation of the detailed junction models.

Table 28:Dimensional variables sizes.

\begin{tabular}{|c|c|c|c|c|c|c|}
\hline $\begin{array}{c}\mathbf{L 1} \\
(\mathbf{m m})\end{array}$ & $\begin{array}{c}\mathbf{L 2} \\
(\mathbf{m m})\end{array}$ & $\begin{array}{c}\mathbf{L 3} \\
(\mathbf{m m})\end{array}$ & $\begin{array}{c}\mathbf{E 1} \\
\mathbf{( m m})\end{array}$ & $\begin{array}{c}\mathbf{E 2} \\
\mathbf{( m m})\end{array}$ & $\begin{array}{c}\text { Junction } \\
\text { Type }\end{array}$ & $\begin{array}{c}\text { Load } \\
\text { direction }\end{array}$ \\
\hline 20 & 20 & 20 & 2 & 2 & $\mathrm{~A}$ & 'Z' \\
\hline 40 & 40 & 40 & 3 & 3 & $\mathrm{~B}$ & $' \mathrm{Y} \mathrm{Y}^{\prime}$ \\
\hline 60 & 60 & 60 & 4 & 4 & & \\
\hline 80 & 80 & 80 & & & & \\
\hline
\end{tabular}

\subsection{RESULTS: DETAILED VS SIMPLIFIED}

Once all the models were calculated, and the results were storaged, it was carried out a qualitative analysis of the relation between simplified and detailed models outcomes in order to extract conclusions of the behavior of the different junction types and load directions from graphical information.

It should be noted that the main goal of the present section is to correct the deviation of the maximum strain outputs of simplified welded junctions models with the aid of the validated detailed ones described in Section 6. For this reason, the following graphs are focused in studying the ratio between the output of both models. This value can be understood as the needed correcting factor to convert the results of the simplified models into the results of the detailed ones. 
Additionally, as it was already stated, beam type element models do not consider local strain raisings due to stiffness changes at the junction. Therefore, these models would be calculating the nominal bending strain according to the Timoshenko beam theory at each node location of the model, which gives very similar outputs to classical formulation for small bending loads of slender beam as is the case. In this scenario, the obtained ratio between detailed and beam models can be considered as an approximation of the SCF tendency of the models.

In the subsequent figures, the aforementioned ratio have been represented separately for the different junction types and load directions. Figure 94 shows the global results of the models, whereas Figure 95, Figure 96 Figure 97 Figure 98 and Figure 99 show the results in relation to the different geometrical variables studied. It was also included an additional graph (Figure 100) to account for a possible combined effect of the relation of the thicknesses (E1/E2). The reason for including this graph arises from the idea that the strains concentrations are originated from steep stiffness changes. According to this, differences in the thicknesses of the profiles might have a local effect on the stiffness change and thus in the SCF.

The following significant characteristics can be extracted from the observation of the graphs:

- Maximum ratios are observed for junctions Type A subjected to load in $Y$ direction, which exceed values of 10 in some cases, while for the rest of configurations the maximums outcomes hardly overtake values of 5 . In this regard, it is noticed that the range of values is also significantly more scatter for this configuration.

- Independently of the junction type or load direction, minimum ratio values are close to 2. It implies that the maximum strains of the detailed models at least doubles the ones of the simplified models. This scenario evidences the impossibility to perform any fatigue analysis based on simplified models without corrective actions.

- The variable L1 has a clear influence in both types of junctions, especially when load is applied in $Y$ direction. Increasing L1 also increases the ratio. This effect is already seen in the global graph for Type A junctions, where four steps belonging to the four different values of L1 are clearly noticed. This effect is much more moderate for load in Z direction, specially for junctions Type B.

- For L2, no significant influence is observed for junctions Type A regardless the load direction. Nevertheless, junctions Type B show a decreasing tendency of the detailed to simplified models relation as $L 2$ increases, when load is applied in $Y$ direction.

- Similarly to L2, variable L3 shows no significant influence for Type A junctions. On the opposite, for Type B junctions, and both load directions, it is noted an increasing tendency of the ratio when L3 increases.

- With respect to the variables related to the thicknesses (E1 and E2), they almost do not alter the ratio of the Type $B$ junctions. Conversely, they do show influence on Type $A$ junctions in both load directions. The effect of E1 is opposite to the effect of E2: while increasing E1 decreases the ratio values of Type $A$ junctions, E2 tends to increase them. 
- The influence of the thicknesses relation is only observed for Type A junctions. Higher ratio values are obtained for small E1/E2 relations, i.e. when E2 taken significantly larger values with respect to $E 1$. It is noted that, although the stiffness change is the same when $E 1 / E 2=0.5$ than when $E 1 / E 2=2$, the junctions show only to be sensitive to this effect in the first case.
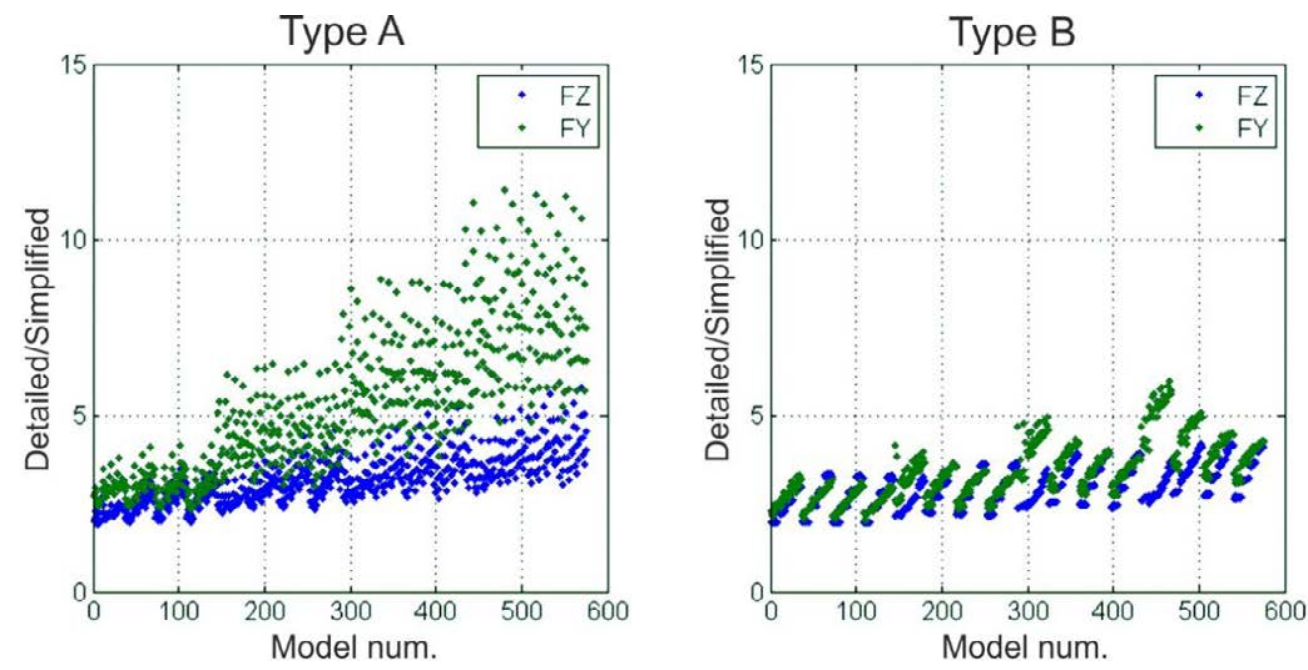

Figure 94 Overall detailed versus simplified models ratio.
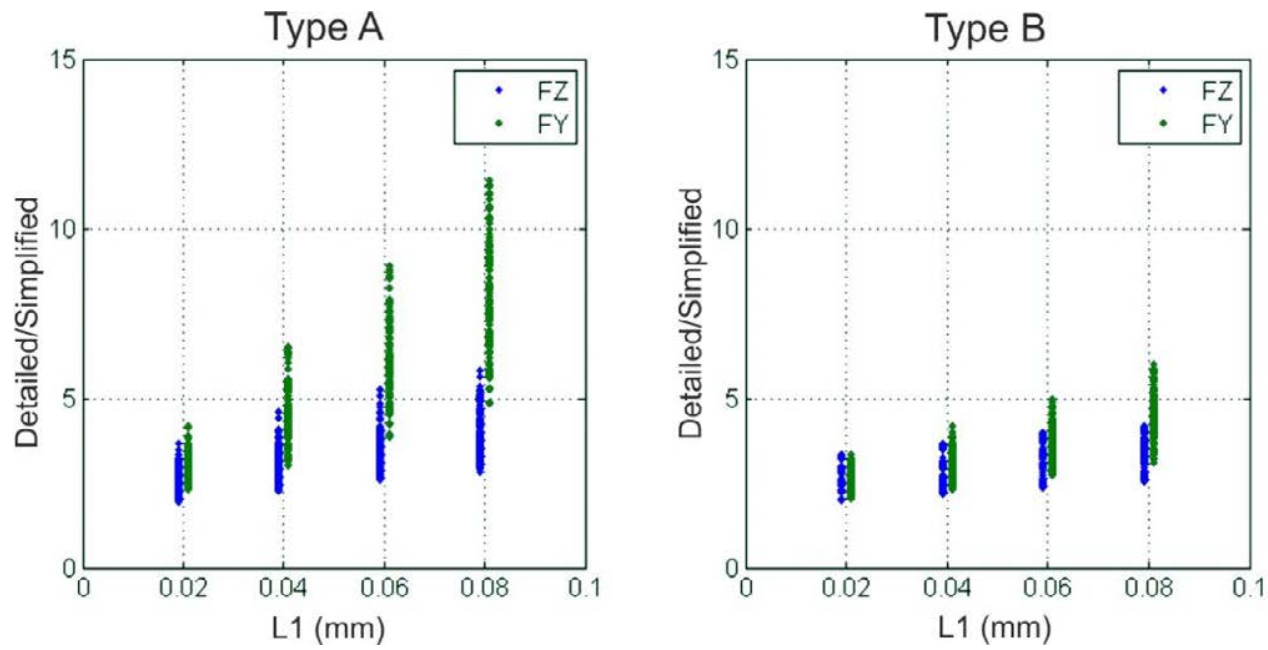

Figure 95 Detailed versus simplified models ratio: relation with L1.
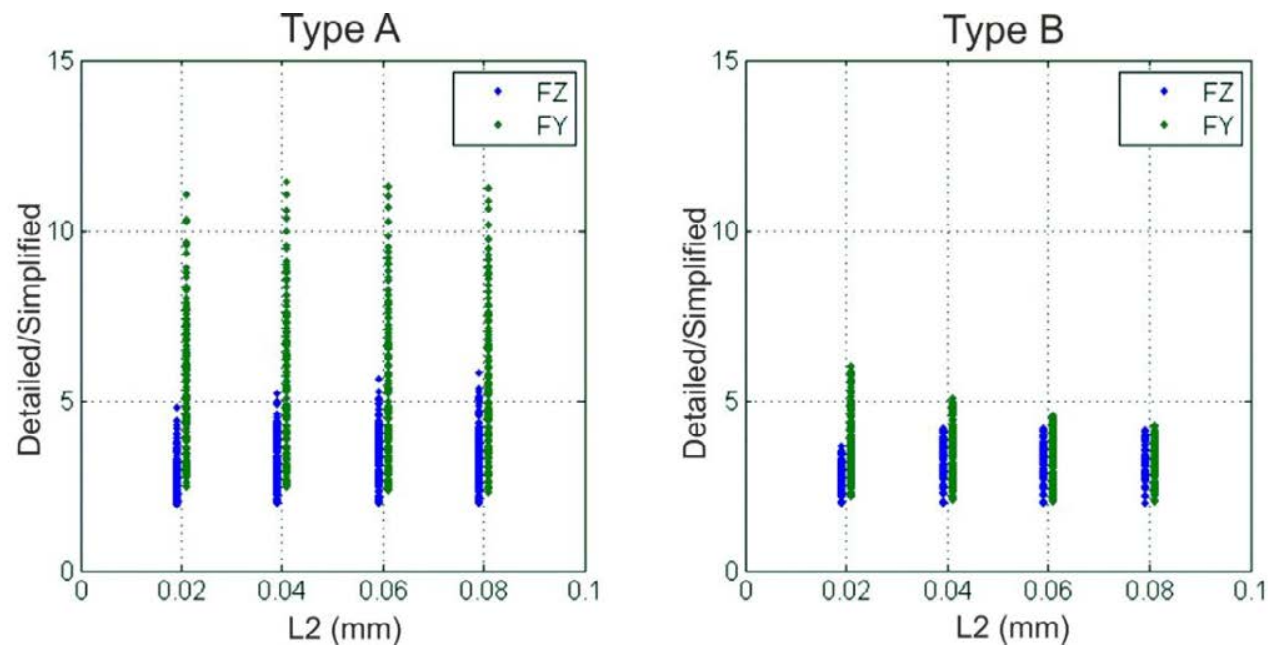

Figure 96 Detailed versus simplified models ratio: relation with L2. 

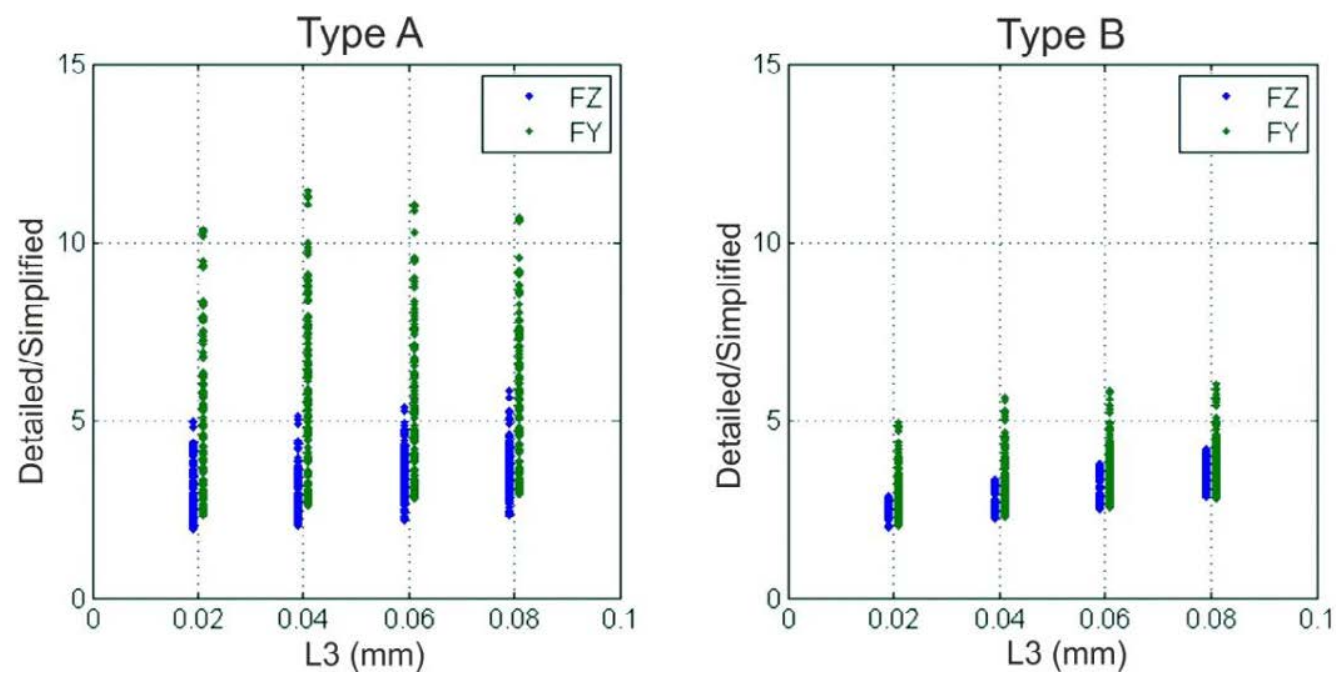

Figure 97 Detailed versus simplified models ratio: relation with L3.
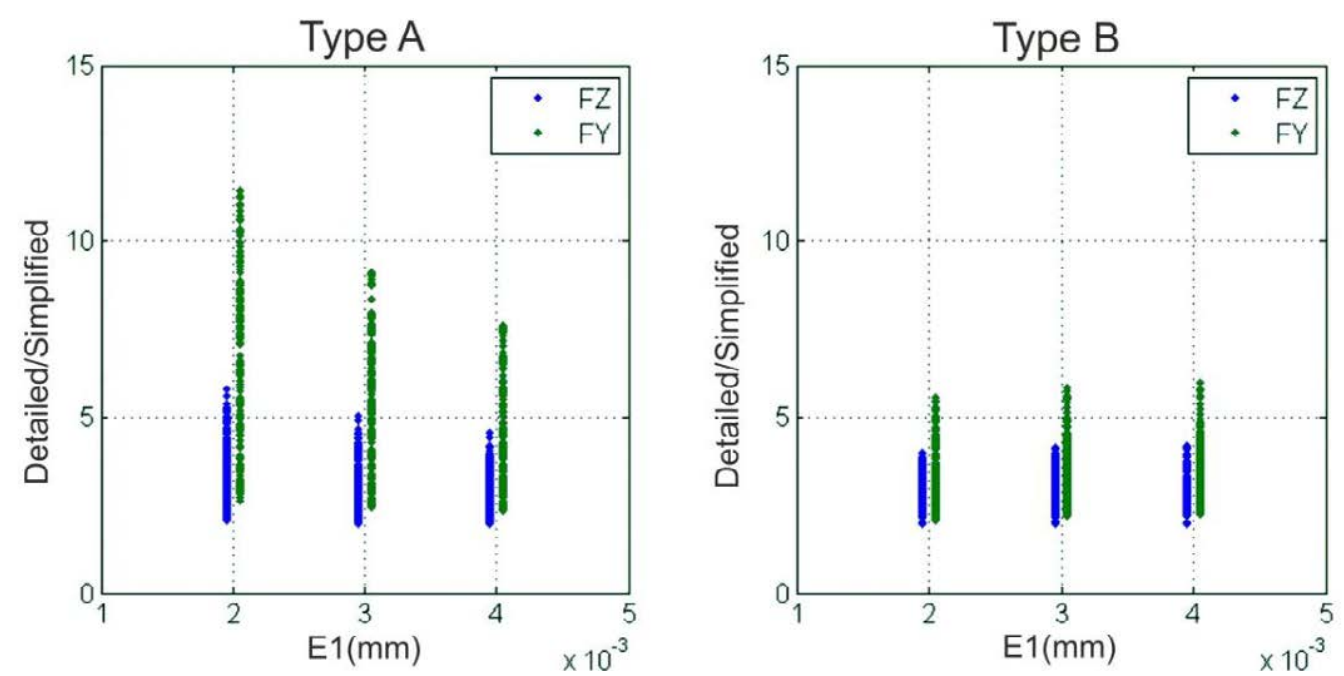

Figure 98 Detailed versus simplified models ratio: relation with E1.
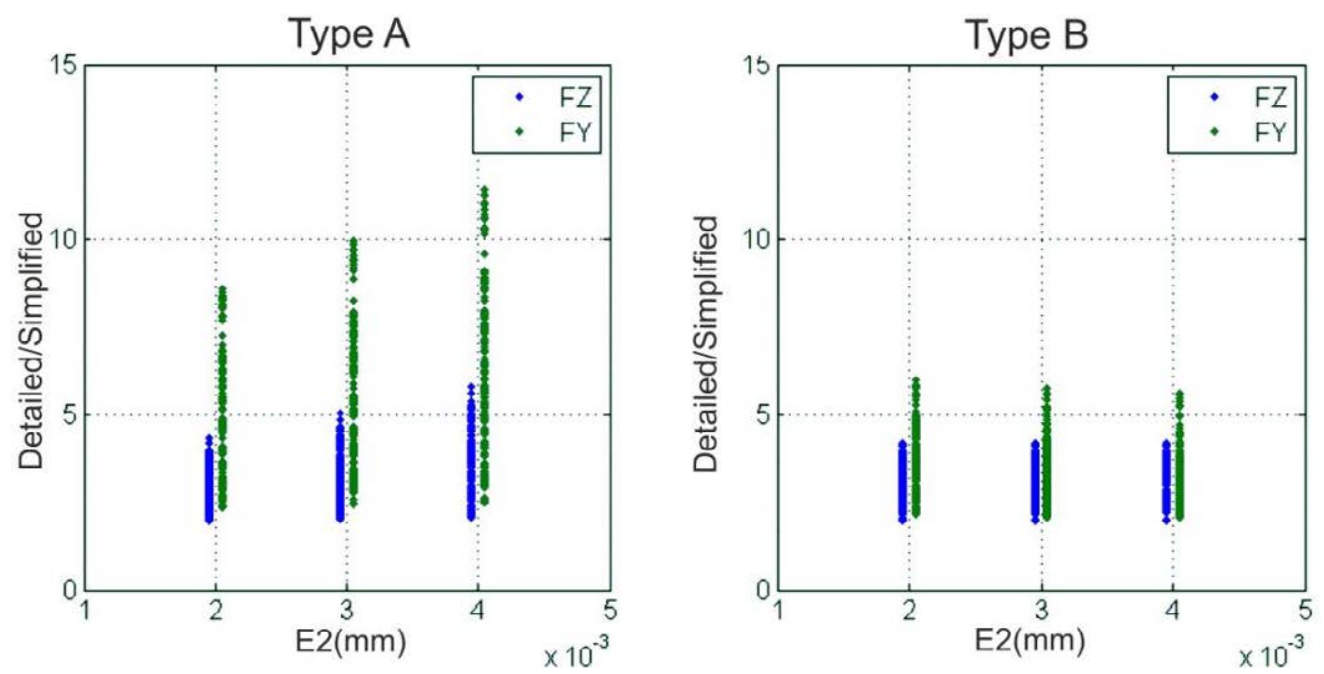

Figure 99 Detailed versus simplified models ratio: relation with E2. 

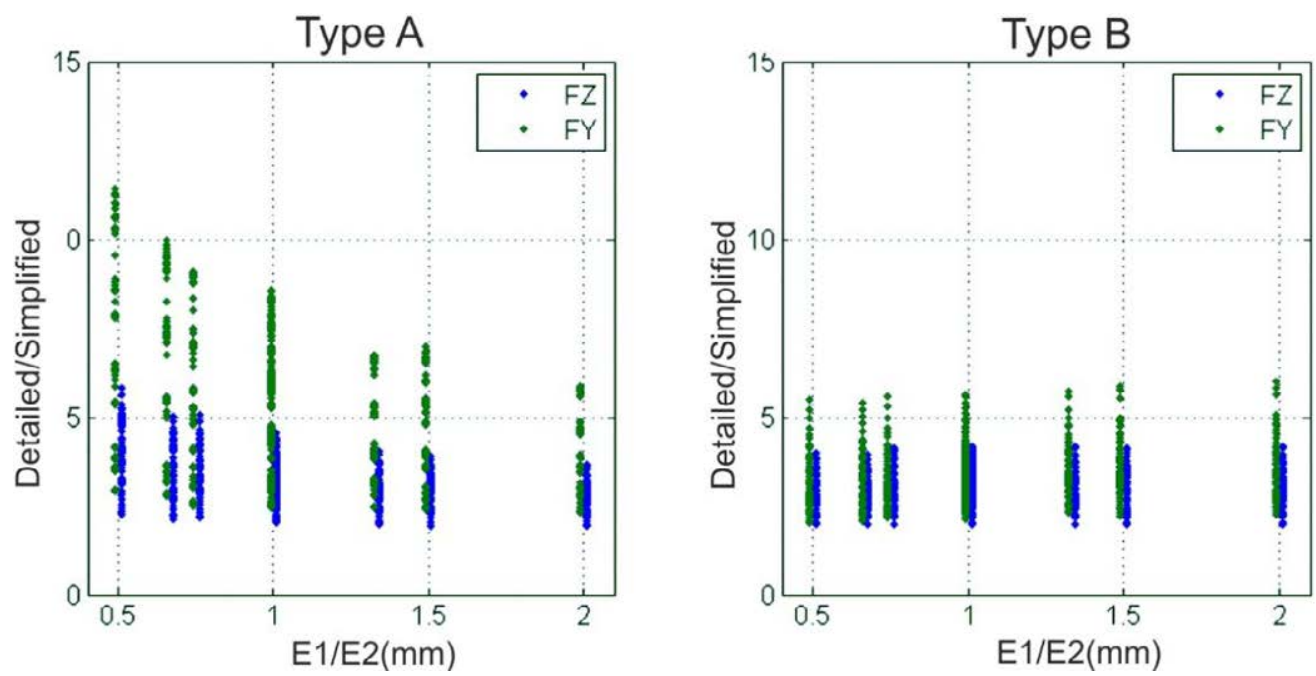

Figure 100 Detailed versus simplified models ratio: relation with E1/E2.

Overall, it can be concluded that the general behavior for Type A junctions differ significantly from the behavior of Type $B$ junctions. Within each type of junction, the influence of the different geometric variables have similar tendencies in both load directions. Nevertheless, in some cases these tendencies are notably more pronounced for one load direction with respect to the other.

In these scenario, it was decided to construct four independent regression models (for each junction type and load direction) in order to avoid adaptability problems due to different behavior under the same geometric conditions. The process of the determination of these regression models for each case is detailed in the following section.

\subsection{REGRESSION MODELS}

The last section covered a qualitative analysis of the influence of the different geometric variables isolated. Nevertheless, it is likely to exist other influences resulting of the interaction of them, more difficult to interpret graphically. Furthermore, the influence of each variable might be linear, non-linear or both.

Since it is not possible to know in advance the geometric variables, or combination of them, that have a significant influence on the response of the junction, the initial regression models should consider a wide number of combinations. Then, those parameters with no significant contribution (i.e. does not induce and increase of the error of the model in case of removal) can be discarded from the model.

In this scenario, the initial model proposed is a multiple non-linear regression model composed by $k=21$ terms as shown in eq. (1.112) and the consequent same number of coefficients $\left(B_{0} \ldots B_{20}\right)$. The first 6 terms are pure linear, followed by 10 interaction linear ones, and 5 more pure quadratic terms at the end. 


$$
\begin{aligned}
& y_{i}=\beta_{0}+\beta_{1} L 1_{i}+\beta_{2} L 2_{i}+\beta_{3} L 3_{i}+\beta_{4} E 1_{i}+\beta_{5} E 2_{i}+\beta_{6} L 1 L 2_{i}+ \\
& +\beta_{7} L 1 L 3_{i}+\beta_{8} L 1 E 1_{i}+\beta_{9} L 1 E 2_{i}+\beta_{10} L 2 L 3_{i}+\beta_{11} L 2 E 1_{i}+ \\
& +\beta_{12} L 2 E 2_{i}+\beta_{13} L 3 E 1_{i}+\beta_{14} L 3 E 2_{i}+\beta_{15} E 1 E 2_{i}+\beta_{16} L 1_{i}{ }^{2}+ \\
& +\beta_{17} L 2_{i}{ }^{2}+\beta_{18} L 3_{i}{ }^{2}+\beta_{19} E 1_{i}{ }^{2}+\beta_{20} E 2_{i}{ }^{2}
\end{aligned}
$$

Where ' $y{ }^{\prime}$ ' is the response (i.e. the relation between detailed and beam models in our case) of the 'ith' FEM model calculated.

Each coefficient ' $\boldsymbol{B}_{k}$ ' of the model is estimated by ' $b_{k}$ ' from the sample data using the method of least squares. The consequent estimated response can be written as:

$$
\begin{aligned}
& y_{i}=\hat{y}_{i}+e_{i}=b_{0}+b_{1} L 1_{i}+b_{2} L 2_{i}+b_{3} L 3_{i}+b_{4} E 1_{i}+b_{5} E 2_{i}+b_{6} L 1 L 2_{i}+ \\
& +b_{7} L 1 L 3_{i}+b_{8} L 1 E 1_{i}+b_{9} L 1 E 2_{i}+b_{10} L 2 L 3_{i}+b_{11} L 2 E 1_{i}+ \\
& +b_{12} L 2 E 2_{i}+b_{13} L 3 E 1_{i}+b_{14} L 3 E 2_{i}+b_{15} E 1 E 2_{i}+b_{16} L 1_{i}{ }^{2}+ \\
& +b_{17} L 2_{i}{ }^{2}+b_{18} L 3_{i}{ }^{2}+b_{19} E 1_{i}{ }^{2}+b_{20} E 2_{i}{ }^{2}+e_{i}
\end{aligned}
$$

Where ' $\mathrm{e}_{i}$ ' is the residual associated with the response if the ith model, or the difference between the ratio calculated by the ith FEM model, and the one estimated by the regression model.

To express it in a compacted manner, let ' $x_{k i}$ ' be the $k$ term of the ith model. Using matrix notation the model can be written as:

$$
y=X b+e
$$

Where ' $y$ ' is an $\mathrm{n} \times 1$ vector with the FEM models results, ' $X$ ' is an $\mathrm{n} \times \mathrm{k}$ matrix of the levels of the regression variables, ' $e$ ' is an $n \times 1$ vector of the residuals and ' $b$ ' is a $k \times 1$ vector of the regression parameters estimates.

$$
\begin{array}{rlrl}
y & =\left[\begin{array}{l}
y_{1} \\
y_{2} \\
\vdots \\
y_{n}
\end{array}\right], & \mathrm{X} & =\left[\begin{array}{ccccc}
1 & x_{11} & x_{12} & \cdots & x_{1 k} \\
1 & x_{21} & x_{22} & \cdots & x_{2 k} \\
\vdots & \vdots & \vdots & \ddots & \vdots \\
1 & x_{n 1} & x_{n 2} & \cdots & x_{n k}
\end{array}\right] \\
e=\left[\begin{array}{l}
e_{1} \\
e_{2} \\
\vdots \\
e_{n}
\end{array}\right], & b=\left[\begin{array}{l}
b_{1} \\
b_{2} \\
\vdots \\
b_{k}
\end{array}\right]
\end{array}
$$


The least squares method involves finding the coefficients estimators ' $b$ ' which minimizes the sum of squared error (SSE) function:

$$
S S E=\sum_{i=1}^{n} e_{i}^{2}=e^{\prime} e=(y-X b)^{\prime}(y-X b)
$$

It can be noticed that SSE might me expressed as:

$$
\begin{aligned}
S S E & =y^{\prime} y-b^{\prime} X^{\prime} y-y^{\prime} X b+b^{\prime} X^{\prime} X b \\
& =y^{\prime} y-2 b^{\prime} X^{\prime} y+b^{\prime} X^{\prime} X b
\end{aligned}
$$

And the estimators ' $b$ ' must therefore satisfy:

$$
\frac{\partial(S S E)}{\partial b}=-2 X^{\prime} y+2 X^{\prime} X b=0
$$

which simplifies to:

$$
X^{\prime} X b=X^{\prime} y
$$

Rearranging this expression, we can write the solution of the regression coefficients estimators as:

$$
b=\left(X^{\prime} X\right)^{-1} X^{\prime} y
$$

As it is deduced from the last expression, the parameters estimators of the regression models can be obtained by solving a set of $k$ equations and same number of unknowns.

Given that the influence of the different parameters of the base regression model are initially unknown, and with the idea of obtaining an optimized solution (i.e. minimized error with the least number of variables), it was decided to evaluate all possible combinations of the said initial regression model. This implies solving a total of $2^{(k-1)}$ different models for each junction type and load direction, that is, $2.1 \mathrm{e}^{6} \times 4=8.4 \mathrm{e}^{6}$ regression models in all.

The total error of each regression model ' $e_{r}$ ' was calculated as the sum if the residuals.

$$
e_{r}=\sum_{i=1}^{n} e_{i}=\sum_{i=1}^{n}\left(y_{i}-X_{(i, 1 \ldots k)} b_{r}\right)
$$

These errors were later normalized with respect to the overall minimum error achieved in each junction type and force direction regression model for comparative purposes.

$$
e_{r(\text { norm })}=\frac{e_{r}}{\min \left(e_{1}, \ldots, e_{2^{(k-1)}}\right)}
$$


The following Figure 101 shows the minimum normalized error obtained in relation to the number of variables used. It should be noted that, for each number of variables, there are different possible combinations of regression models, depending on the variables discarded. Since, only the combination with the smallest error is of interest, the rest are automatically discarded due to the higher error shown using the same number of variables.

Figure 101 shows clearly how the error decreases rapidly for lower number of variables used, and notably slower or even inexistent when the number of variables approach to the maximum. This evidences a compromise between model simplicity and accuracy. Additionally, it is observed that the error decrease behaves differently depending on the junction type and load direction.

In this scenario, it was decided to choose the regression models of each junction type and load direction as function of the normalized error, independently of the number of variables. It was selected the model whose achieves a normalized error under 1.05 with the least number of variables. It means that the overall error of the selected regression will be less than $5 \%$ with respect to the overall best regression model registered among the $2.1 \mathrm{e}^{6}$ calculated. Selected models for each type are highlighted with green circles on the graphs of Figure 101, and the values of the parameters estimators are gathered in Table 29, where the three parameters with the highest estimator have been turned bolt.

\section{' $Y$ ' direction}

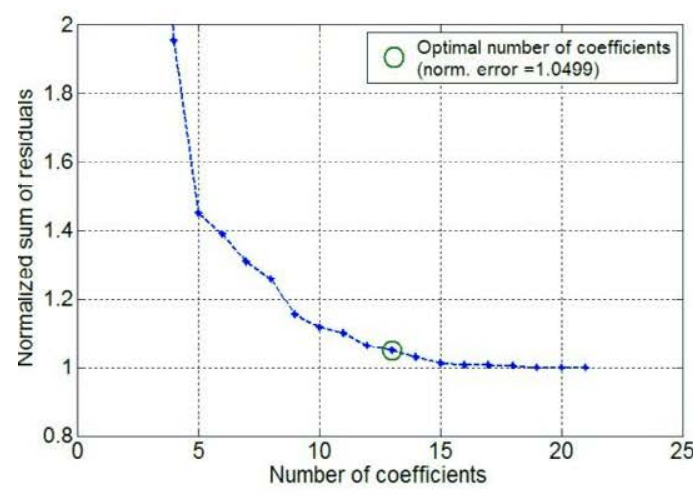

\section{Type A}

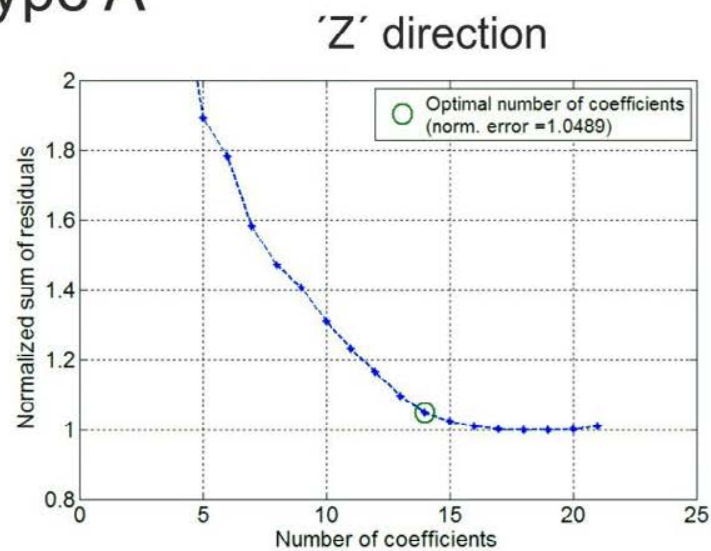

Type B

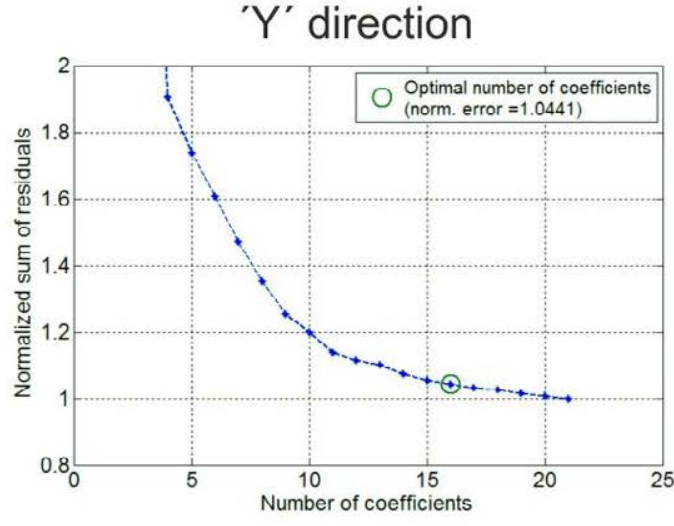

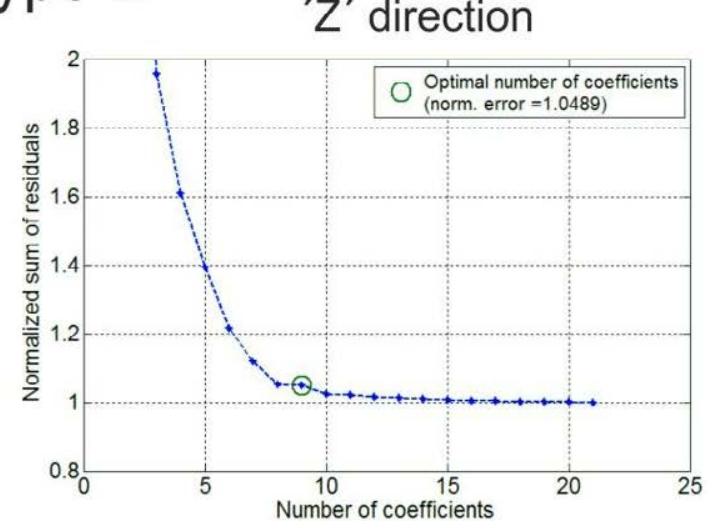

Figure 101 Regression models normalized sum of residuals. 
Table 29:Parameter estimators of the selected regression models.

\begin{tabular}{|c|c|c|c|c|c|}
\hline Variable & $\begin{array}{l}\text { Parameter } \\
\text { Estimator }\end{array}$ & $\begin{array}{c}\text { Type A } \\
\text { 'Y' Load }\end{array}$ & $\begin{array}{c}\text { Type A } \\
\text { 'Z' Load } \\
\end{array}$ & $\begin{array}{c}\text { Type B } \\
\text { 'Y' Load } \\
\end{array}$ & $\begin{array}{c}\text { Type B } \\
\text { 'Z' Load }\end{array}$ \\
\hline--- & $b_{0}$ & 0.55 & 1.04 & 2.07 & 1.11 \\
\hline L1 & $b_{1}$ & 101.05 & 26.68 & 31.22 & 7.71 \\
\hline $\mathrm{L} 2$ & $b_{2}$ & & 15.88 & -17.44 & 20.69 \\
\hline L3 & $b_{3}$ & & & 16.88 & 11.14 \\
\hline E1 & $b_{4}$ & & & 106.66 & \\
\hline E2 & $b_{5}$ & 386.02 & & -281.16 & \\
\hline $\mathrm{L} 1 * \mathrm{~L} 2$ & $b_{6}$ & & & -328.00 & 84.00 \\
\hline L1*L3 & $b_{7}$ & & -117.36 & 105.78 & \\
\hline L1*E1 & $b_{8}$ & -23257.37 & -5057.65 & & \\
\hline $\mathrm{L} 1 * \mathrm{E} 2$ & $b_{9}$ & 12946.04 & 5846.29 & -1364.98 & \\
\hline $\mathrm{L} 2 * \mathrm{~L} 3$ & $b_{10}$ & 183.95 & 216.52 & -22.49 & 138.42 \\
\hline L2*E1 & $b_{11}$ & & -1448.93 & -567.61 & \\
\hline L2*E2 & $b_{12}$ & & 2254.89 & 2447.85 & \\
\hline L3*E1 & $b_{13}$ & 6320.79 & & & 905.02 \\
\hline L3*E2 & $b_{14}$ & 5977.95 & & 2443.39 & -287.18 \\
\hline E1*E2 & $b_{15}$ & -237619.65 & -103238.29 & & \\
\hline $\mathrm{L}^{2}$ & $b_{16}$ & 78.67 & & 130.02 & \\
\hline $\mathrm{L}^{2}$ & $b_{17}$ & -72.72 & -201.24 & 177.40 & -264.52 \\
\hline $\mathrm{LS}^{2}$ & $b_{18}$ & -362.94 & 49.12 & -130.47 & \\
\hline$E 1^{2}$ & $b_{19}$ & 105702.40 & 62260.83 & & \\
\hline$E 2^{2}$ & $b_{20}$ & & 35996.41 & & \\
\hline
\end{tabular}

From the observation of the results, it is noticed that the regressions of Type $A$ junctions clearly show the highest estimators for variables related to the thicknesses of the sections, denoting a significant influence of these geometrical variables on the ration between detailed and beam models. This effect was already seen during the qualitative analysis (Figure 100)

On the contrary, the estimators of the Type 'B' junctions are more uniformly distributed and there is not such clear tendency. For load in ' $Y$ ' direction, although not very significant, the models seems to be specially sensible to the relation between E2 thickness and the different lengths of the junctions cross section. This effect is not appreciable for $Z$ load direction, where no special sensitivity to any geometrical variable can be remarked.

With the purpose of assessing the accuracy of the selected models, the FEM results where compared to the regression predictions (Figure 102), and the relative difference between them(Figure 103), for each junction where calculated as follows:

$$
e_{\text {rel }(i)}=\frac{y_{i}-X_{(i, 1 \ldots k)} b_{r}}{y_{i}} \quad i=(1 \ldots n)
$$

Figure 102, which mainly has a qualitative interpretation, states the general good agreement of the select models to the FEM results. 


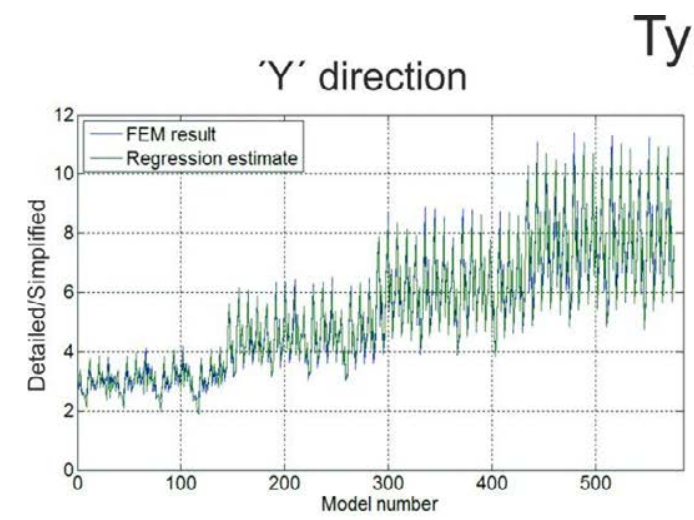

Type A
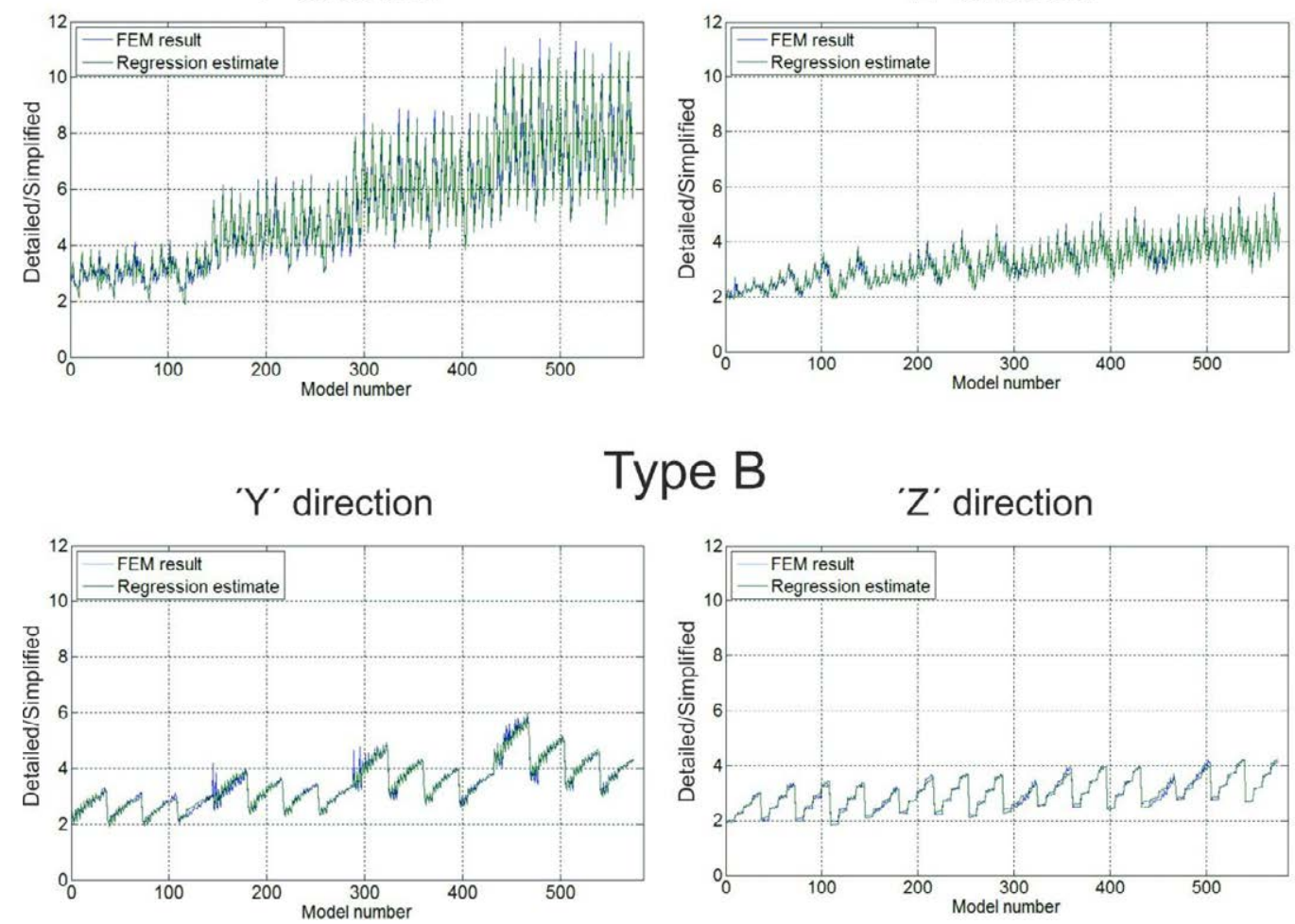

Type B

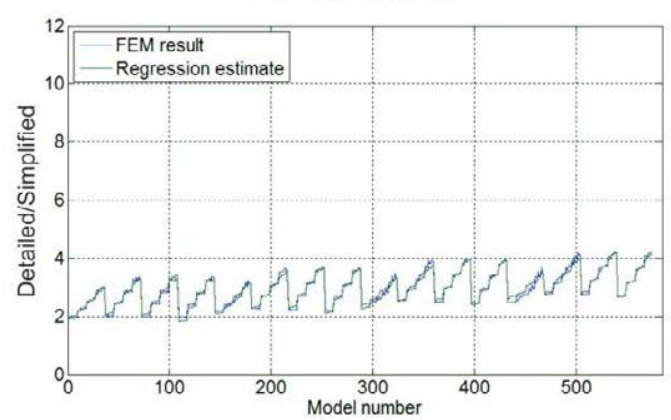

Figure 102 Output of FEM models versus regression estimations.

' $Y$ ' direction

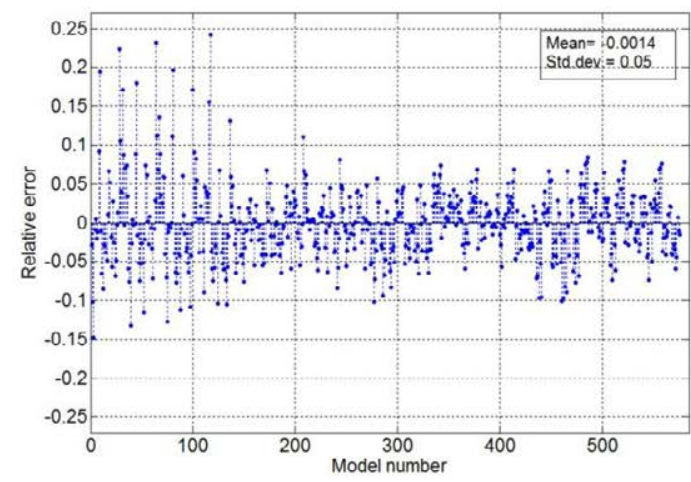

' $\mathrm{Y}$ ' direction

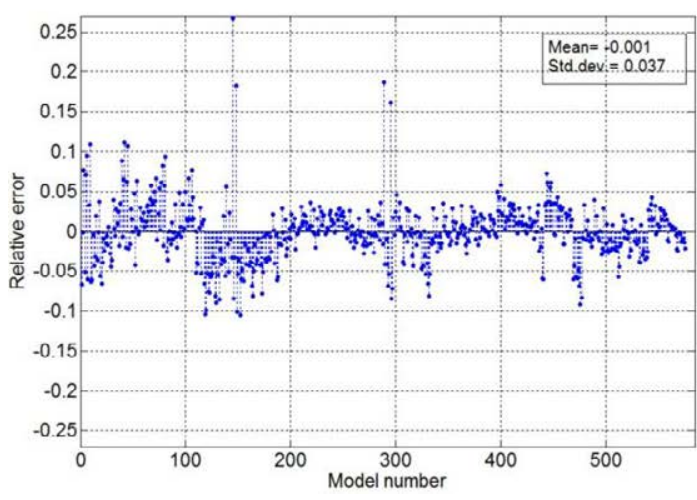

Type A

' $Z$ ' direction

\section{Type B}

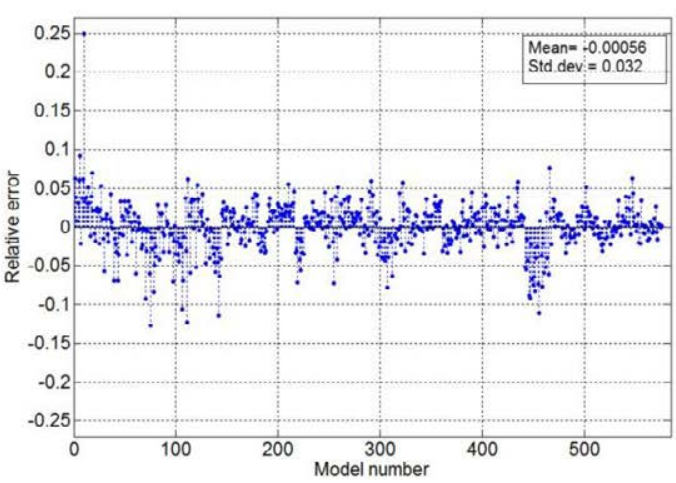

'Z' direction

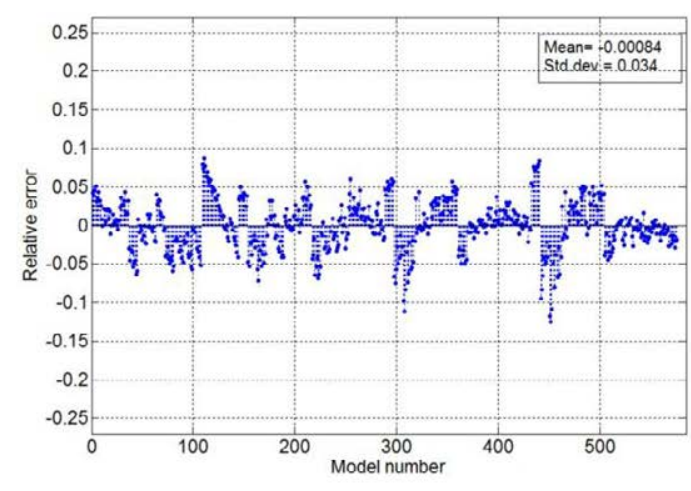

Figure 103 Relative error of regression estimations. 
From the observation of Figure 103, it is noticed that the mean of the errors is very close to zero in all junctions, so no general under or overestimation tendency of the values is present on the models. The scatter of the error is very similar for junctions Type $B$ in both load directions and for junction Type A loads in ' $Z$ ' direction, where the standard deviation rounds 0.035 . It supposes and approximate error of under $7 \%$ in $95 \%$ of the calculated junctions following the assumption that they follow normal distributions. Junction Type A loaded in ' $Y$ ' direction shows a slightly higher dispersion, so an equivalent confidence interval of $95 \%$ would lead to an error of around $10 \%$. It should be pointed out that this type of junction already shows significantly higher dispersion with respect to the rest of the cases, which may hinder the adaptability of the model.

Finally, it should be noted that the deviations of the regression models are in any case well below the scatter nature of the SCF itself, whose standard deviation was proven to be around one order of magnitude higher $(\sigma=0.354)$ in Section 5.2.4. In this scenario, the errors of the regressions are of low significance in comparison to the scatter behavior of the actual junctions themselves.

\subsection{VALIDATION OF THE REGRESSION MODELS}

Although an important number of possible junction combinations have been used to construct the regressions and asses the accuracy, different geometries might be used in the design of LPTV structures. For this reason, it is important that the regression are capable to predict the behavior of any welded junction, regardless the geometry of the section of the profiles.

In order to assess the accuracy of the regressions for geometries different from those used for their construction, a series of new validation cases were calculated using all possible combinations of the dimensions listed in Table 30. Less common section sides of 50 and $70 \mathrm{~mm}$, and thicknesses of 2 and $4 \mathrm{~mm}$ were evaluated. It can be noticed that the thicknesses were already employed in the initial calculations. Nevertheless, they were chosen since it is extremely uncommon to find different values from 2,3 or $4 \mathrm{~mm}$ in LPTV structures.

Table 30:Validation models dimensions.

\begin{tabular}{|c|c|c|c|c|c|c|}
\hline $\begin{array}{c}\mathbf{L 1} \\
(\mathbf{m m})\end{array}$ & $\begin{array}{c}\mathbf{L 2} \\
(\mathbf{m m})\end{array}$ & $\begin{array}{c}\mathbf{L 3} \\
(\mathbf{m m})\end{array}$ & $\begin{array}{c}\mathbf{E 1} \\
\mathbf{( m m})\end{array}$ & $\begin{array}{c}\mathbf{E 2} \\
(\mathbf{m m})\end{array}$ & $\begin{array}{c}\text { Junction } \\
\text { Type }\end{array}$ & $\begin{array}{c}\text { Load } \\
\text { direction }\end{array}$ \\
\hline 50 & 50 & 50 & 2 & 2 & $\mathrm{~A}$ & $' \mathrm{Z}$ ' \\
\hline 70 & 70 & 70 & 4 & 4 & $\mathrm{~B}$ & $' \mathrm{Y}$ ' \\
\hline
\end{tabular}

In total, there were calculated $2^{7}=128$ detailed and $2^{6}=64$ simplified models for the validation. As already mentioned, simplified models cannot account the differences between Type A and Type B junctions, therefore the total amount of models is one half of the detailed ones. Figure 104 shows the values obtained from the calculations as well as the predictions of the regressions for each junction type and load direction. Great agreement between both is noticed in all cases. In accordance to the initial models, Type A junction loaded in ' $Y$ ' direction show the highest and more disperse outcomes. 


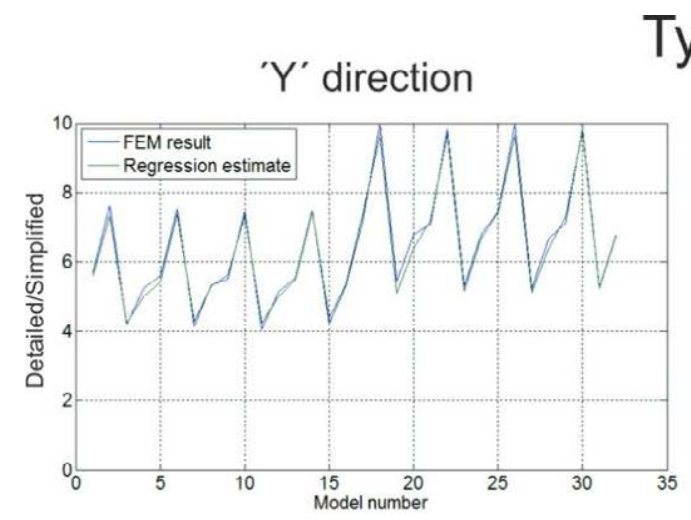

Type A

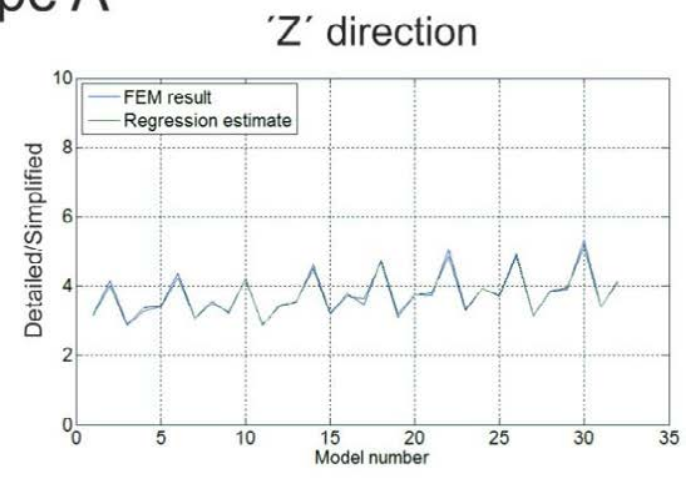

' $Y$ ' direction

Type B
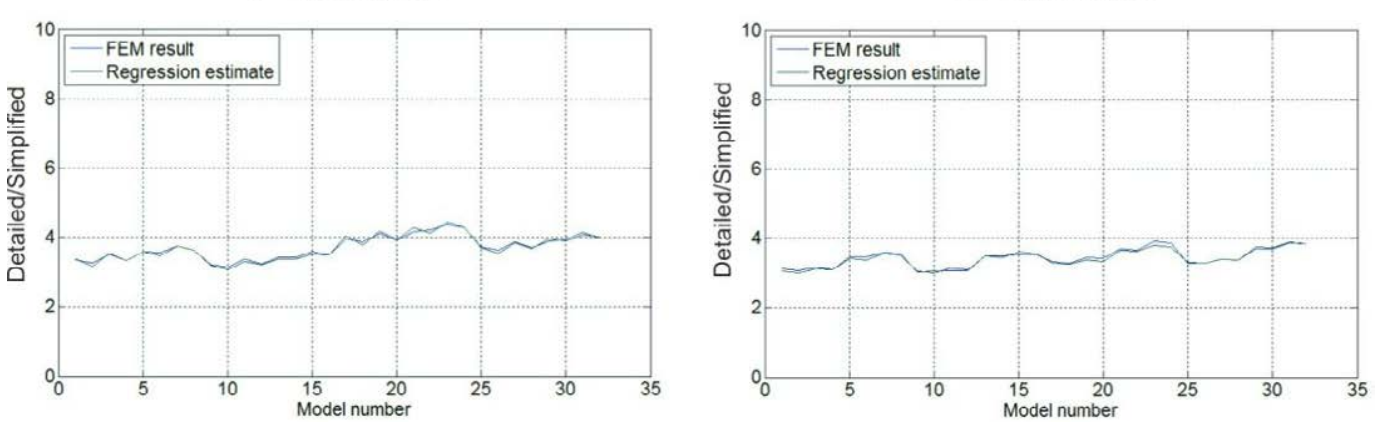

Figure 104 Output of FEM models versus regression estimations for validation cases.

Type A

' $Y$ ' direction

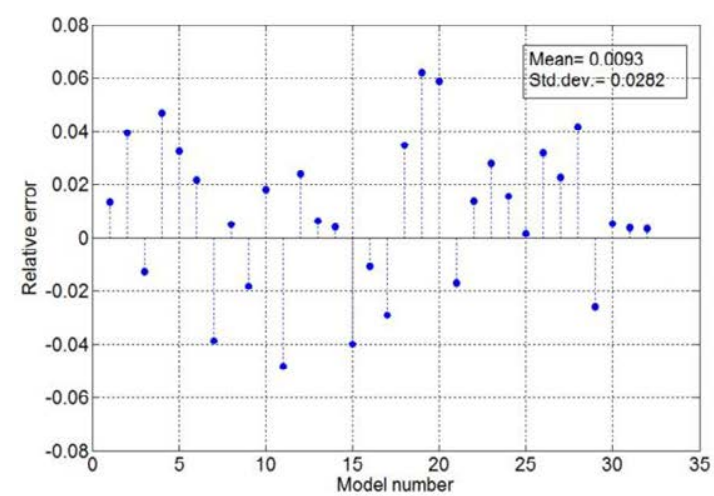

'Z' direction

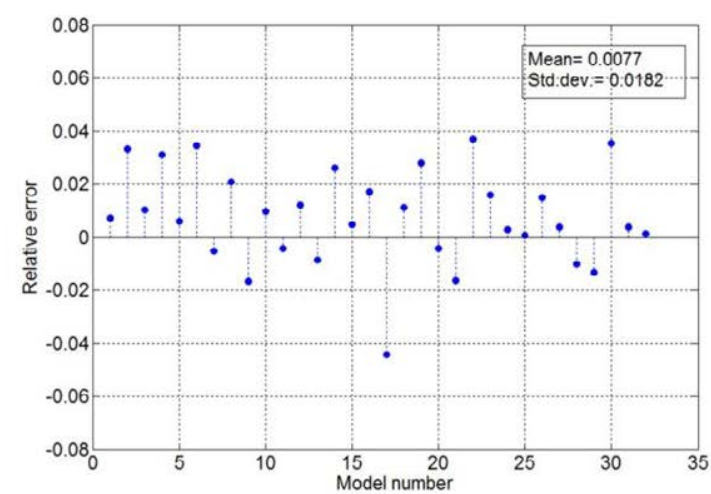

Type B

' $Z$ ' direction

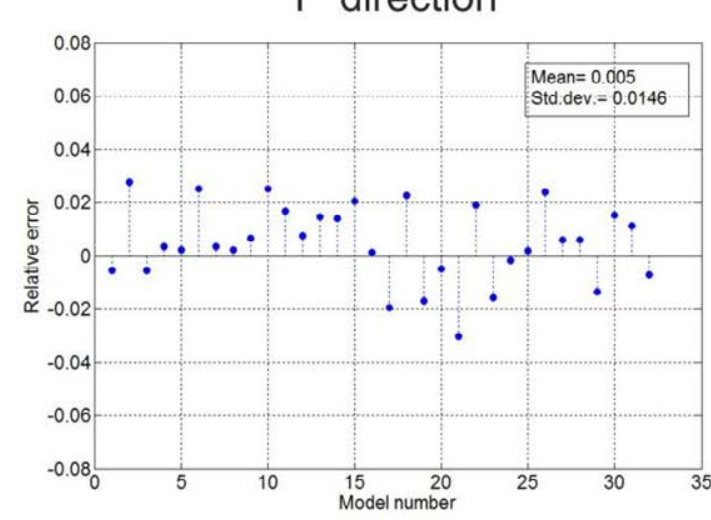

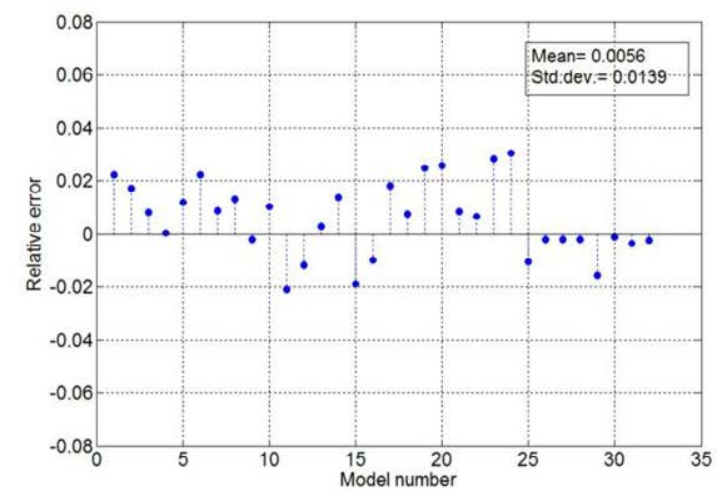

Figure 105 Relative error of regression estimations for validation cases. 
Equivalently to the initial calculations, the relative error of the regression predictions with respect to the FEM results obtained for the validation cases was employed to assess the accuracy according to the expression of eq.(1.123). The obtained results are shown in Figure 105 , which evidences the accuracy of the models regardless the geometry of the junction.

It is observed that the standard deviation of the error is smaller than those of the general regression models, showing that the regressions behaves more accurately for this sample than the overall average for the sample used in this section, Once again, the model of the Type A junctions in $\mathrm{Y}$ direction shows slightly higher deviations than the rest of the models.

Conclusively, it has been proven in this section the capacity of the regression models constructed to correct the outputs of ' $T$ ' welded junctions calculated by means of beam elements. They will be therefore included in the routine of fatigue life prediction of LPTV structures as correcting factors of the beam element type maximum strain outputs. 


\section{APPLICATION OF THE FATIGUE LIFE PREDICTION METODOLOGY TO A LPTV STRUCTURE: CASE STUDY}

Among the former sections, the proper actions to study and characterize the different aspects of the methodology of fatigue life prediction presented in Figure 1 have been carried out.

The results obtained provide the complete necessary information to assess the fatigue behavior of bus LPTV 'T' type junctions. Nevertheless, the evaluation process requires significant data flow and processing that would be very costly if it is not supported by some sort of automation routines.

This section presents the application of the complete methodology over an LPTV example model and provides the routines employed for this purpose. It should be pointed that there are many other possible routines to develop the methodology, although they should always employ the data and conditions presented throughout the thesis, and follow the structure of Figure 1.

First, an initial routine uses Ansys $^{\circledast}$ to apply the accelerations cycles determined in Section 4 (Figure 49, Figure 50, and Figure 51) and export the nominal strain data over the selected ' $T$ ' type junctions. Such exported data is thereafter processed in a Matlab ${ }^{\circledR}$ based script that provides as a final output the expected damage on each junction during the established lifespan of the LPTV. Finally, a new Ansys ${ }^{\circledR}$ routine plots the damage of each junction over the FEM model for a better localization of critical zones or profiles. Routines constructed in Ansys ${ }^{\circledR}$ use the self programming language APDL (Ansys Parametric Design Language).

The process followed in the application of the methodology as well as the routines employed and the results obtained are presented in further detail in the following subsections.

\subsection{LPTV FEM MODEL DESCRITION.}

The bus model used for the application of the methodology is an 12 meter, 2 axle urban bus supplied by INSIA. The complete FEM model is shown in Figure 106. It can be clearly noticed that 1D beam type elements have been used to construct the structural body of the bus. The ground and the seats of the bus have been modeled using shell type elements, the engine and auxiliary components by means of volume type elements. Also, the suspension arms were modeled using volume elements, and link type elements to simulate the pinned connection to the body of the LPTV. The purpose of these components is to provide the proper mass distribution to the bus, and therefore there is no interest in the outcomes of these elements. Consequently, relatively coarse mesh can be employed. It should be pointed out that the model used represents simply an example to show the process of application of the methodology, and thus the results obtained cannot be extrapolated to other LPTV structures of similar characteristics. Nevertheless, some tendencies of the results are expectable to be similar for different designs as will be shown alter. 
The model consists of a total of 19596 volume elements, 27092 shell elements and 10850 beam elements. In accordance with the simplified models of the regressions, BEAM188 element type was used to mesh the beam elements of the bus body. Also same element size of $3 \mathrm{~mm}$ was employed. On the other hand, SHELL63, SOLID45 and LINK8 element types, available in the Ansys element type library, were used to mesh shell, solid and link components respectively.

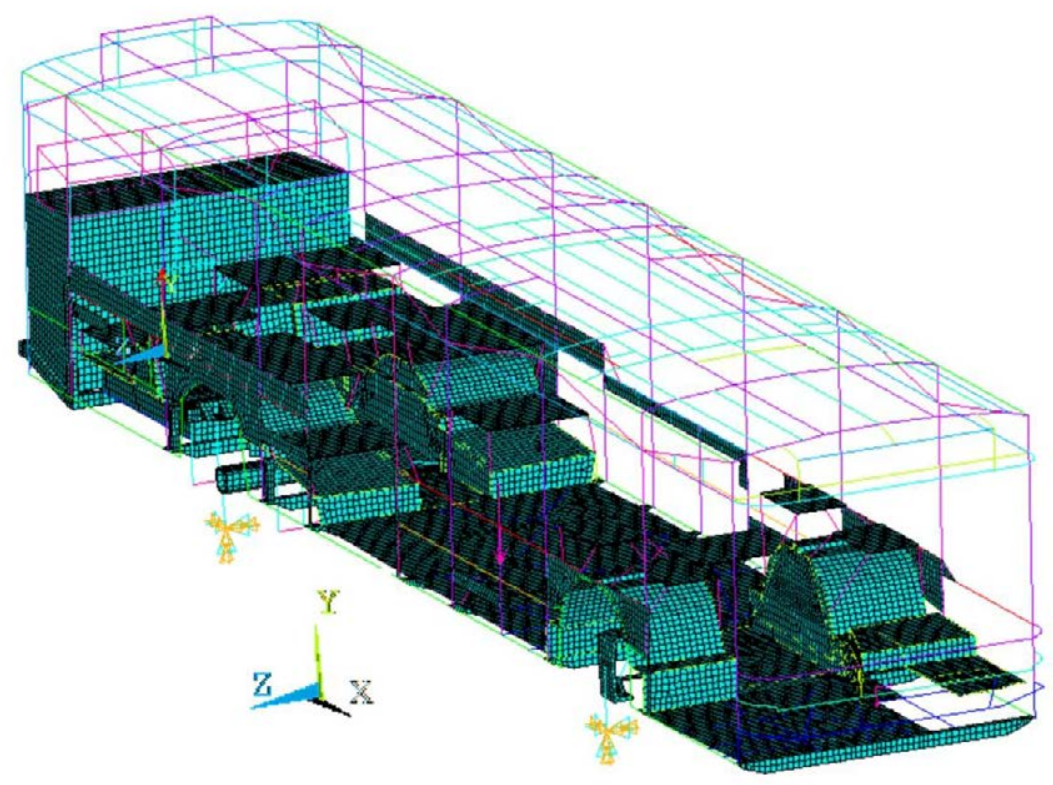

Figure 106 Complete bus FEM model.

The different colors of the beam elements represent the different cross sections of the bus structure. In all, 14 different cross section profiles were used to construct the model which are summarized in Table 31. The nomenclature used to name the two sides and the thickness of the sections was chosen in accordance to the one used in Section 7 for the construction of the regression models.

Table 31:Cross sections of the LPTV structure.

\begin{tabular}{|c|c|c|c|}
\hline Section & $\begin{array}{c}\mathbf{L 1} \\
(\mathbf{m m})\end{array}$ & $\begin{array}{c}\mathbf{L 3} \\
(\mathbf{m m})\end{array}$ & $\begin{array}{c}\mathbf{E 2} \\
(\mathbf{m m})\end{array}$ \\
\hline 1 & 40 & 40 & 3 \\
\hline 2 & 40 & 60 & 3 \\
\hline 3 & 40 & 50 & 4 \\
\hline 4 & 40 & 80 & 3 \\
\hline 5 & 40 & 80 & 4 \\
\hline 6 & 40 & 40 & 4 \\
\hline 7 & 40 & 30 & 3 \\
\hline 8 & 50 & 80 & 3 \\
\hline 9 & 40 & 20 & 2 \\
\hline 10 & 40 & 30 & 2 \\
\hline 11 & 40 & 40 & 2 \\
\hline 12 & 40 & 60 & 2 \\
\hline 13 & 60 & 40 & 2 \\
\hline 14 & 20 & 20 & 2 \\
\hline
\end{tabular}


Regarding the material, it was shown in the previous sections that plastification occurs at the vicinity of the welded junctions as a consequence of the strain concentrations, and given the beam type elements are unable to account for this local raising, linear behavior of the material is expected. It should be kept in mind that in any case, if strains beyond yield points are registered in the evaluated junctions, low fatigue life is expected and a design review is strongly recommended. A young modulus of E=2.02GPa (in accordance of the findings of material characterization of Section 5) and a Poisson coefficient v=0.3 was chosen. Again it should be pointed that the model presented supposes only an example, and thus any typical material stiffness characteristics could be chosen, whose typical values in common structural steels used in LPTV oscillate in the approximate range of $2 \mathrm{GPa}$ to $2.1 \mathrm{GPa}$.

\subsection{LOAD CYCLES APPLICATION: RAINFLOW DATA}

Having defined the model to be evaluated, the first part of the routine is performed in Ansys $^{\circledR}$. The function of the routine is schematized in Figure 107, it performs of the application of the acceleration cycles found in Section 4 and posterior exporting of the strains outputs obtained on the beam type elements of the complete LPTV model of each junction evaluated.

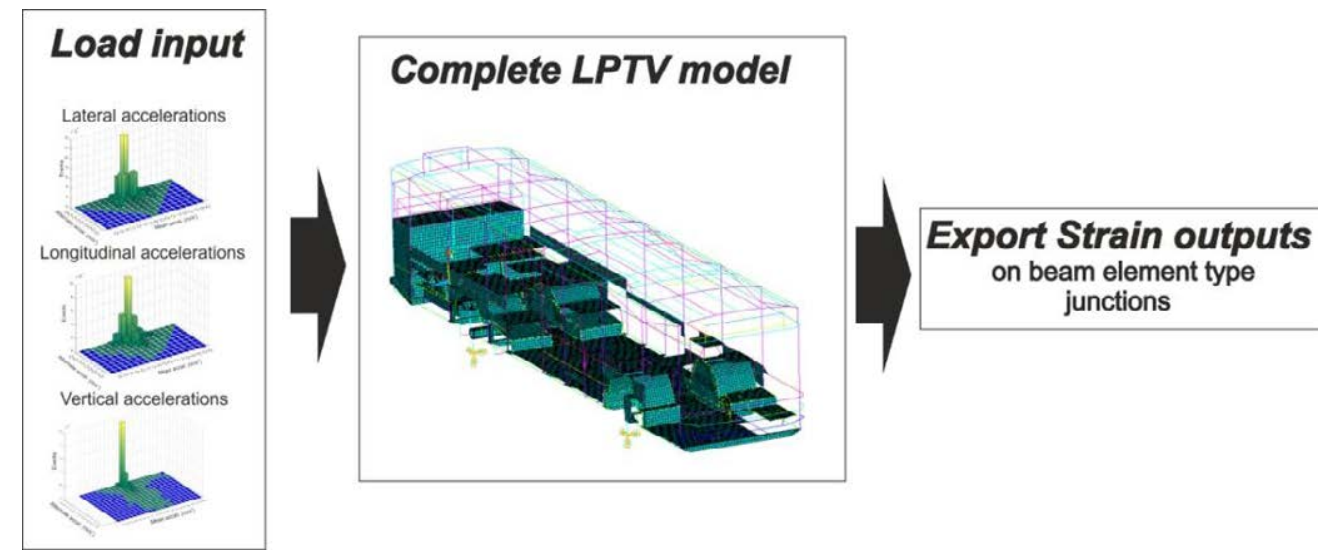

Figure 107 Load cycles application and beam strain output export routine schema.

Since the assessment of the strain concentration behavior, and the correlation regression between simplified and detailed models has been performed over ' $T$ ' type junctions, the routine will be centered in these type of junctions. In all, 73 welded junctions were evaluated in the present model.

Additionally, whereas bending loads during the junction characterization of previous sections were applied only on one of the branches of the welded profiles, these junctions are most likely to suffer bending loads from the four sides that compose it in a real LPTV structure. Consequently, fatigue damage failure can be located at any of these four sides of the junctions. It can be noted that peak strains will be located in different zones depending on the junction side, and thus damage should be treated independently.

In order to ensure proper traceability in terms of junction type and load direction in each junction over the different steps of the routine, it has been adopted the following notation rule schematized in Figure 108. It will allow to distinguish between the different types (A or B) and 
load directions at any of the four sides of the junction. According to this notation, a Type A junction will be considered a junction whose continuous pillar is aligned with ' $X$ ' direction both for lateral and top/bottom junctions, always preserving the coordinate system of the model presented in Figure 106. On the contrary, a Type B junction will have the continuous pillar aligned with ' $\mathrm{Y}$ ' direction for lateral junctions, and with ' $Z$ ' direction for junctions on the roof and ground of the bus.

The four sides of each junction were also named in order to keep them differenced over the routine. In this sense, 'Back' and 'Forth' sides makes distinction between junctions sides aligned with ' $X$ ' direction, depending on its relative ' $X$ ' position to the junction node (green dot in Figure 108). In the same manner, for junctions placed in the bus laterals, 'Up' and 'Down' side names will distinguish the sides aligned with ' $Y$ ' directions. Analogously, 'Right' and 'Left' sides differentiate sides of the top/bottom junctions aligned with ' $Z$ ' direction. It can be noted that this convention represents the position at which each junction side would lie with respect to the junction node for a viewer placed at the coordinate system origin, and facing towards the bus positive $\mathrm{X}$ direction.

Finally, with regard to the force directions, 'F1' represents forces in a direction equivalent to the force applied in ' $Z$ ' direction according to the notation used for the characterization of the strain concentration of the previous sections. Similarly, 'F2' will be in accordance to the force applied in ' $Y$ ' direction in the previous sections notation.

\section{Lateral junctions}

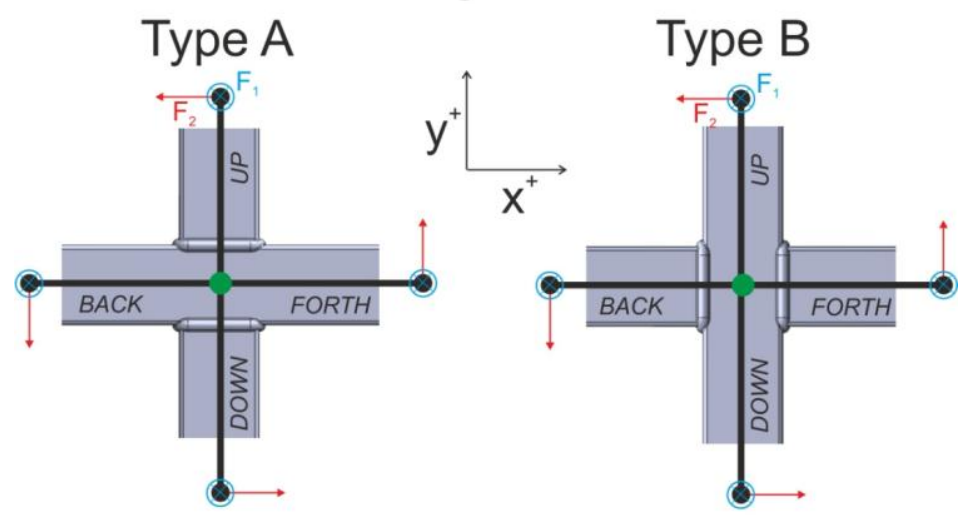

Top/Bottom junctions

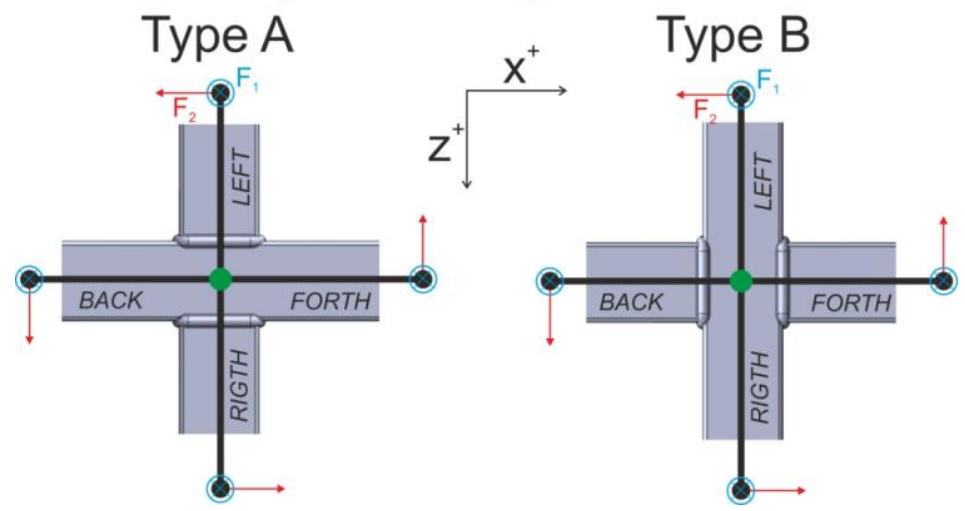

Figure 108 Junction type and load direction notation at each junction side. 
The APDL scripts that performs the load cycles application and output exporting for the case of lateral accelerations are shown in Figure 109 and Figure 110 respectively. These routines can be easily adapted to apply acceleration cycles in longitudinal and vertical directions by changing the direction of load application in lines 27 and 29 of the script, and also the mean and alternate acceleration vectors of lines 3-4 and 6-7 in the case of vertical accelerations in accordance to the Rainflow chart of Figure 51. Obviously, for the complete evaluation of the bus structure fatigue behavior, the three acceleration directions must be considered. 


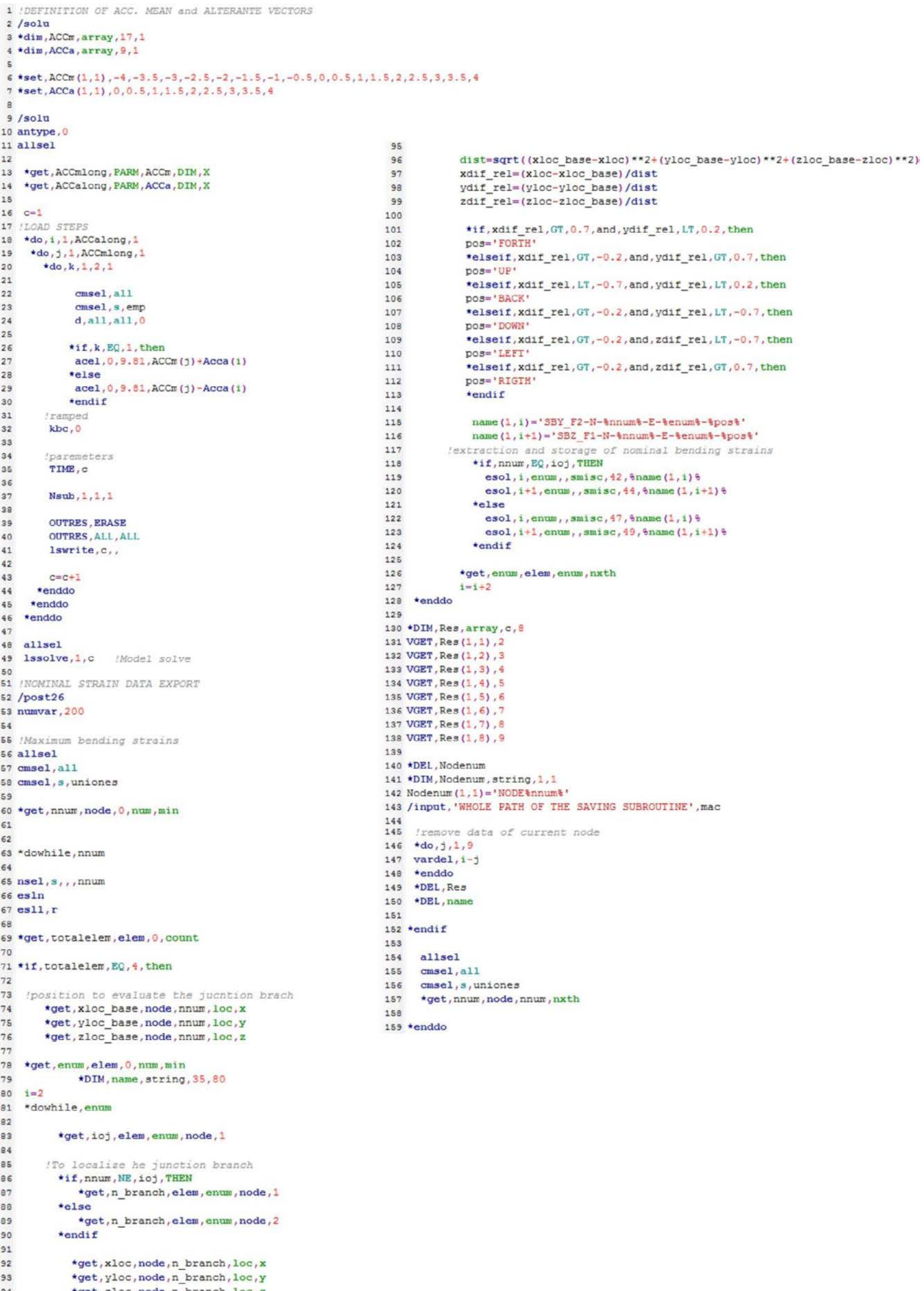

Figure 109 Load cycles application routine for the case of lateral accelerations. 


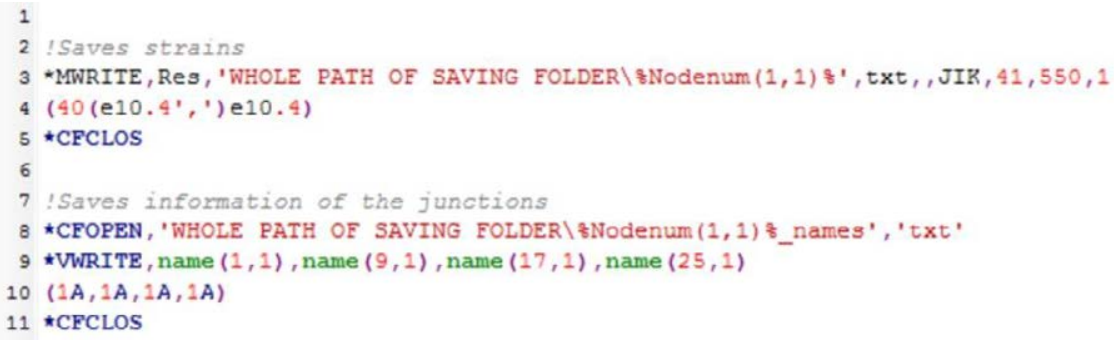

Figure 110 Junction data export sub routine.

In order to ensure proper running of the routines, the following settings should be ensured:

- The nodes of the junctions to be evaluated should be stored in a group named 'uniones' in Ansys prior running the routine.

- To ensure proper execution of data exporting, in line 143 of load application routine, the whole path were the data export routine is placed should be written. Also, in the data export routine itself, the whole path were the data is to be stored should be input in lines 3 and 9 .

The routines will output two text files for each node in the specified output path. By way of example, Figure 115 shows the format and correspondence between these files for node number 8845. The first file contains the strain results for each side and load directions of the junction. The corresponding junction side and load direction of each column is provided in the second file, were each of the eight rows gives information of the corresponding column of the first file.

The rows of the strain output files are the results of each load case. the number of load cases will be in turn twice the number of different acceleration cycles obtained in the Rainflow study, since each cycle has a minimum and a maximum acceleration value that should be evaluated.

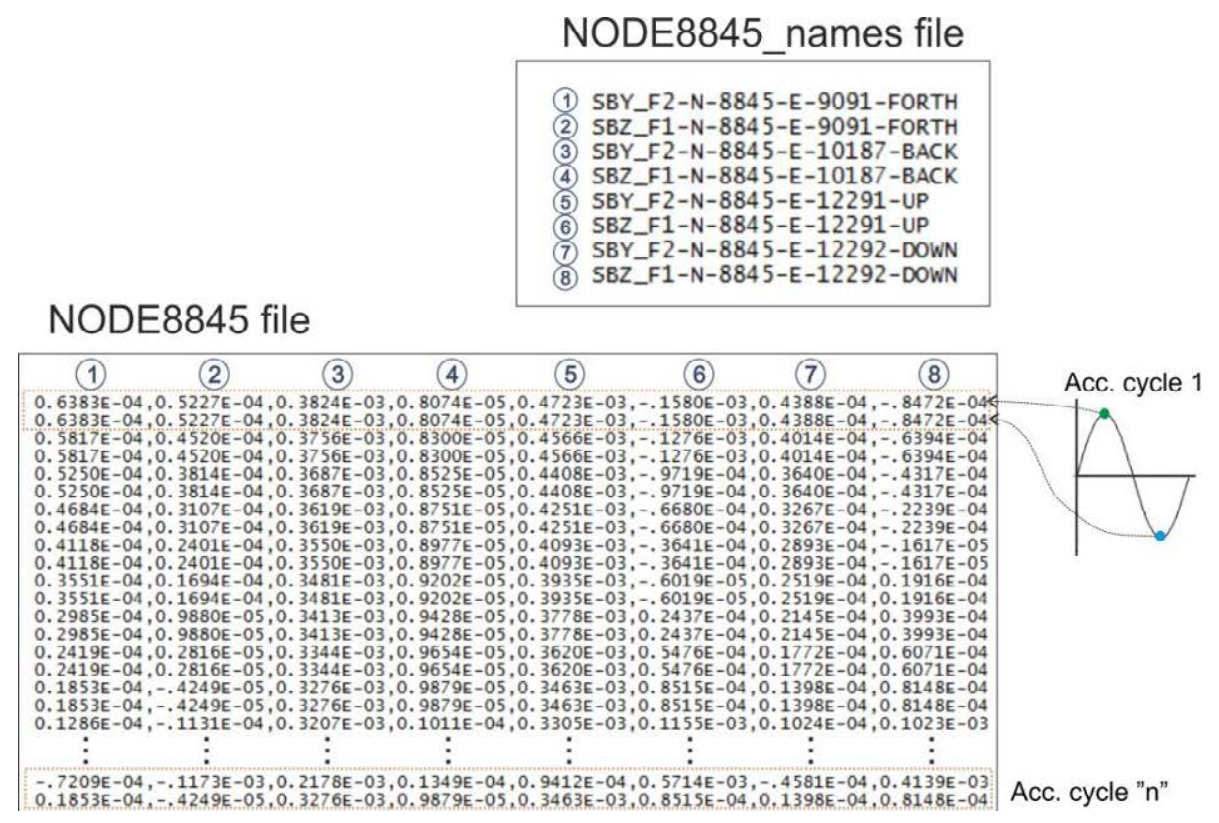

Figure 111 Ansys routine output files. 
The calculation time of each load case oscillates between 10 and 13 seconds in a PC with a 6 core AMD T-1100 processor and 16Gb of RAM memory. Given that lateral and longitudinal calculations consist of 307 load cases, and vertical ones of 546 load cases, the total time for solving would be around 1 hour for lateral and longitudinal acceleration, and between 1.5 and 2 hours in the vertical case. It supposes an overall calculation time of less than 4 hours for all load cycles in all directions.

\subsection{FATIGUE DAMAGE EVALUATION}

Having obtained the corresponding text files with the necessary information of the junction nominal strains, the post processing procedure to evaluate the fatigue damage is developed by means of the main Matlab ${ }^{\circledR}$ script presented in Figure 115 and schematized in the following flow diagram.

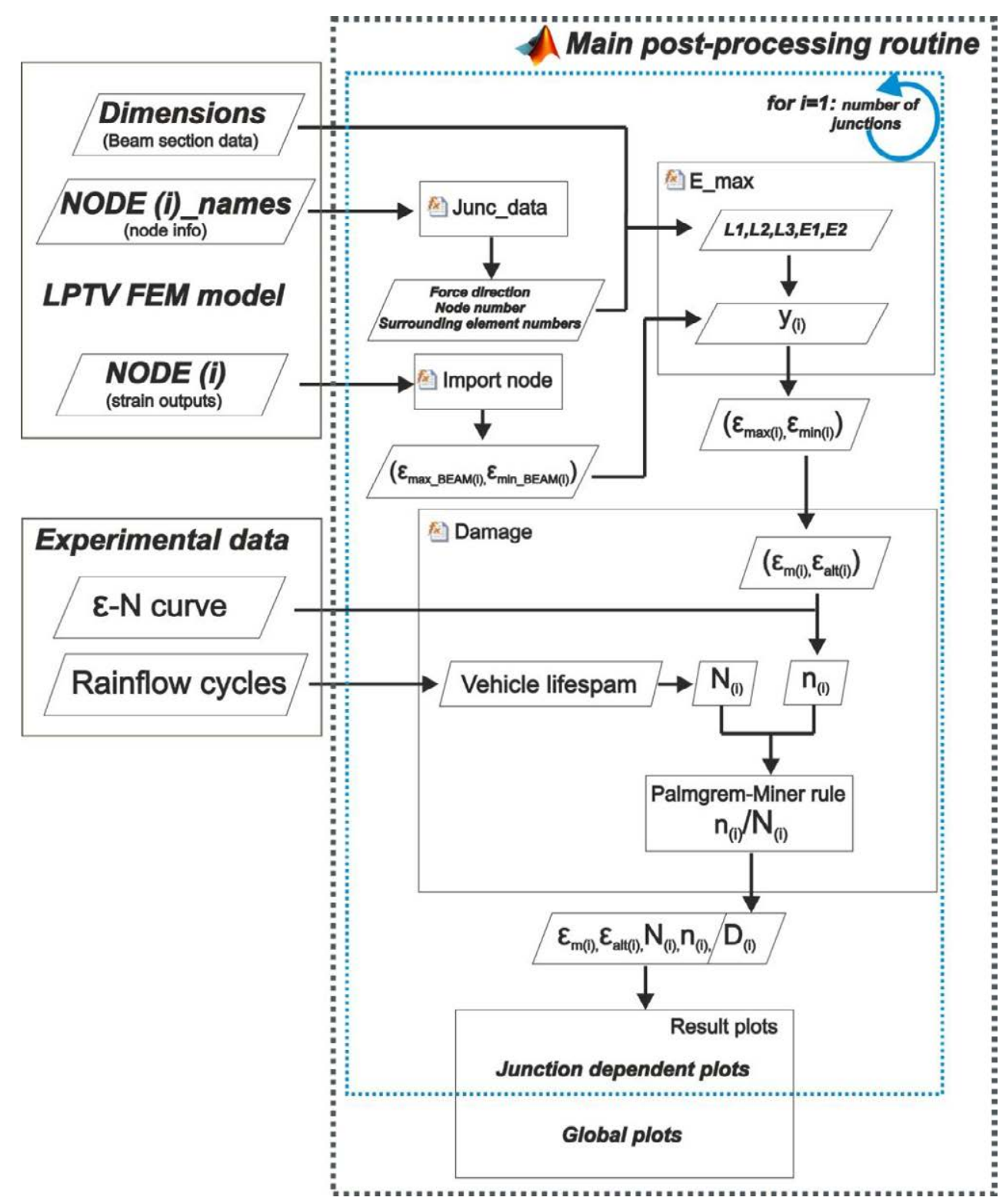

Figure 112 Main fatigue post processing routine flow diagram. 
This principal routine gives structure to the fatigue damage evaluation process, and calls the proper subroutines or functions to perform specific actions during this process. It first obtains the necessary information about the junctions that will be used during the different steps of the evaluation process by calling the 'Junc_data' function. Thereafter, it calls the function 'Import node' to read the nominal strain data, and subsequently the function 'E_max' to determine the corrected maximum strain by applying the correcting factors obtained from the regression models. Finally, it resorts on the function 'Damage' to evaluate the fatigue damage that these strains suppose to each side of the junction. Once the damage evaluation is done, the script performs a series of plots to provide clear and visual output information. All this process will be performed for lateral, longitudinal and vertical accelerations, and inside a loop which will have as many iterations as junction or nodes to be evaluated.

Functions 'Junc_data' and 'Import file' scripts are shown in Figure 113 and Figure 114 respectively. Functions 'E_max' and 'Damage', due to their higher complexity and involvement with the methodology on the thesis will be presented in further detail in the subsequent sections

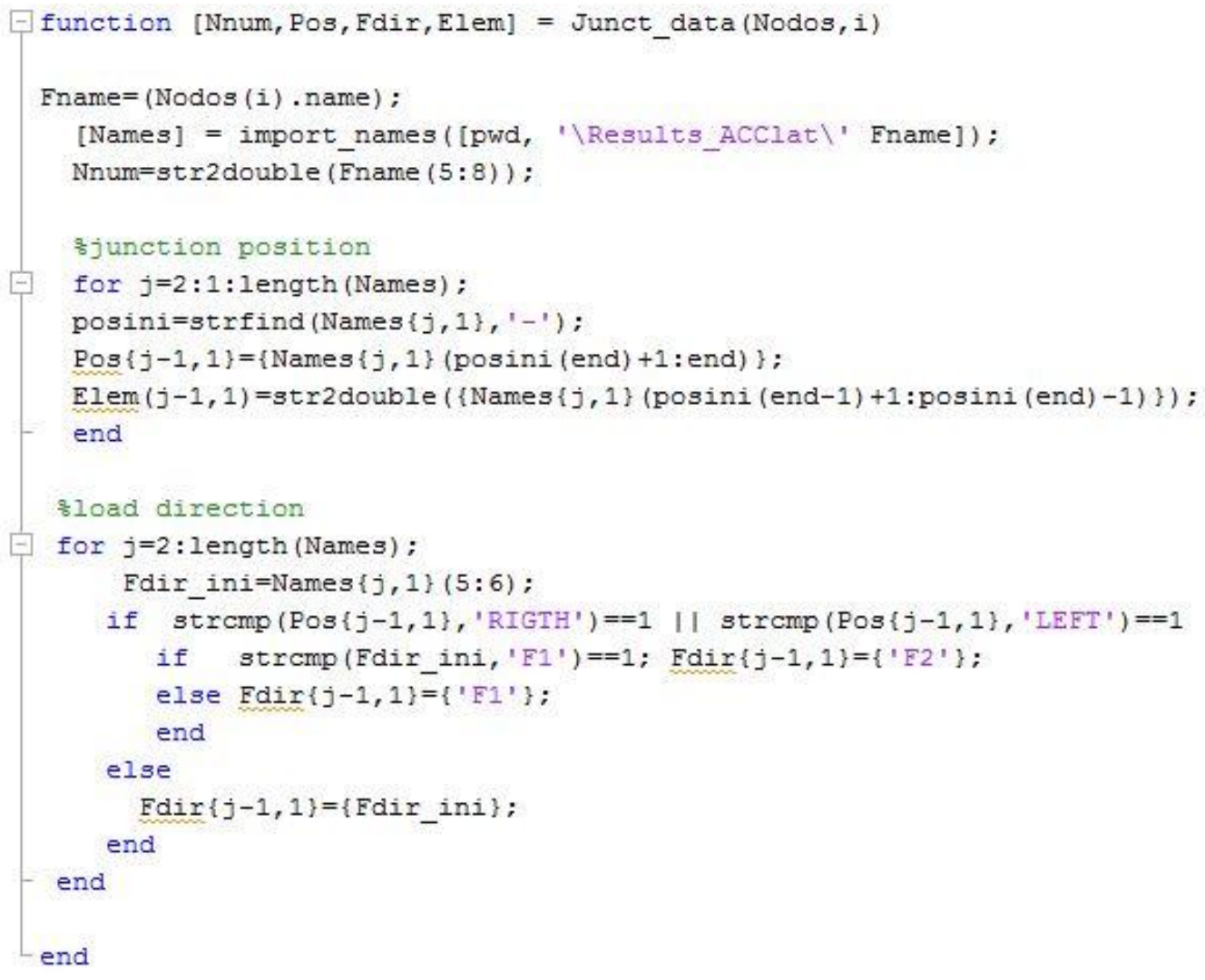

Figure 113 'Junc_data' function. 


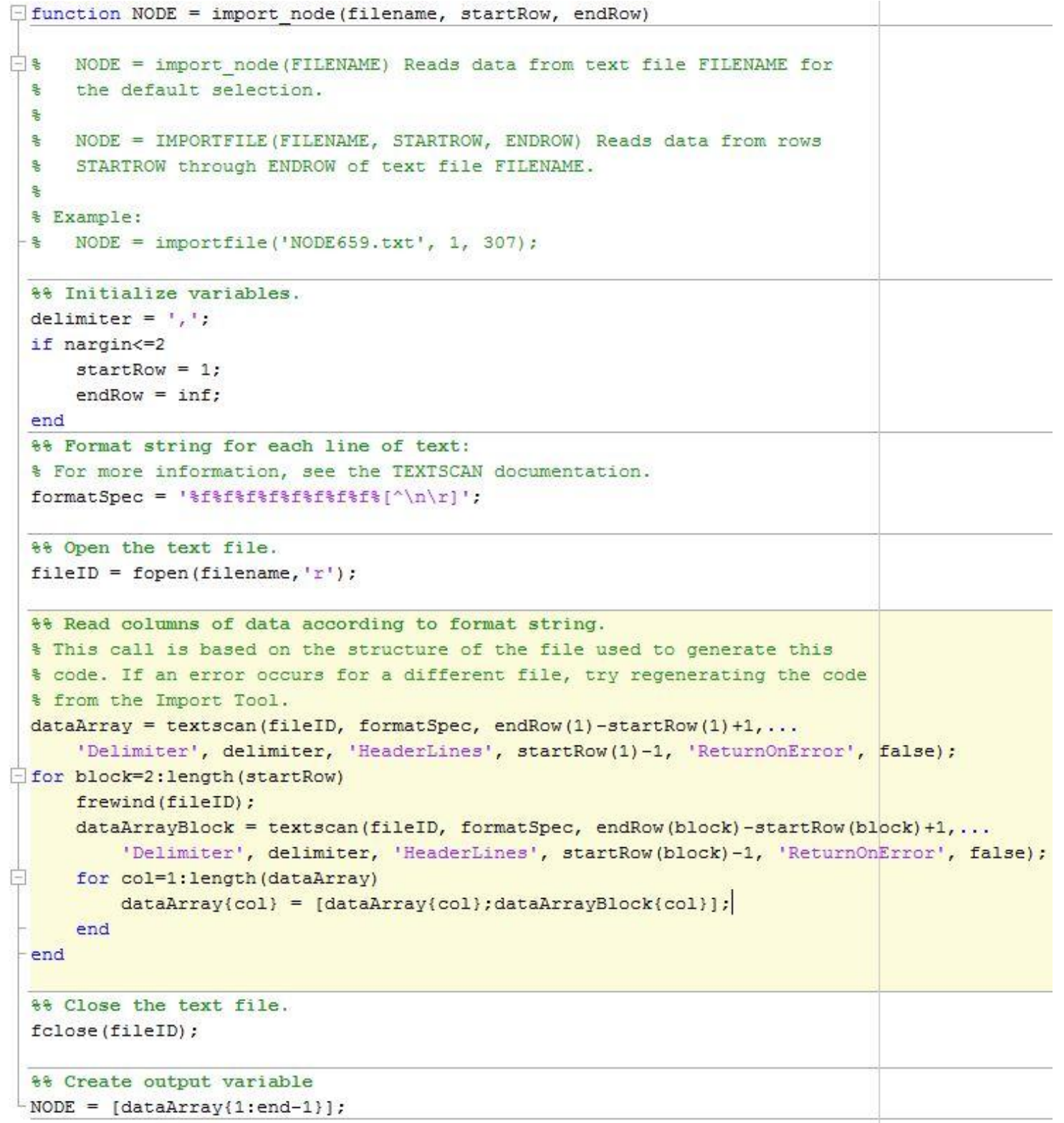

Figure 114 'import_node' function.

In order to ensure proper running of the script, the following settings should be carried out:

- Copy the folders of the nominal strains outputs to the same folder were the main routine and the functions are situated.

- Create an excel file named 'Dimensions.xlsx' with two sheets: one named Elist, with the information of the section number associated to each element evaluated, and the other with a list of the beam cross sections used in the FEM model.

- Create a folder named 'Graphs', where all the figures of the routine will be automatically stored. 


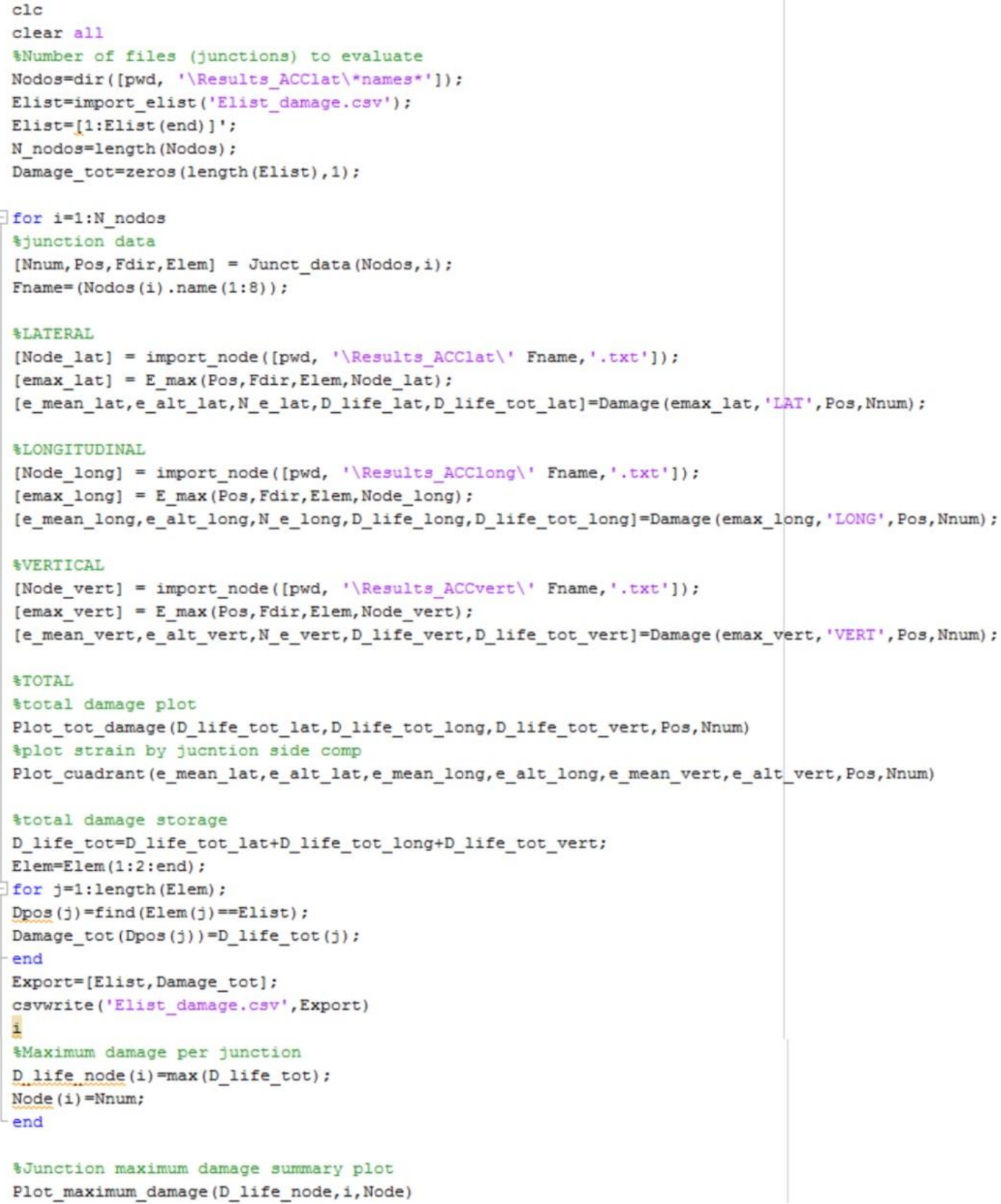

Figure 115 Main fatigue post processing routine script.

\subsubsection{MAXIMUM STRAINS DETERMINATION: REGRESSION MODELS}

The function with the task of determining the corrected maximum strains from the nominal stains output obtained in the simplified model is named 'E_max', and is called three times en each loop iteration of the main routine, in lines 17, 22 and 27 for lateral, longitudinal and vertical accelerations respectively.

The scripting of this function is provided in Figure 116. As inputs, it needs, on one side the matrix with the nominal strains, and on the other the information of the positions and load direction of each side of the junction.

The function first identifies the corresponding regression model that should be applied to each junction side and load direction. Subsequently, uses the information of the 'Dimensions' 
excel sheet to determine the dimension of the cross sections of each junction side. Finally, it carries out the maximum strain correction using the regression models obtained in Section 7 and the local nonlinear SCF compensation according to the regression equations shown in Figure 70 , and provides it as an output to the main routine.

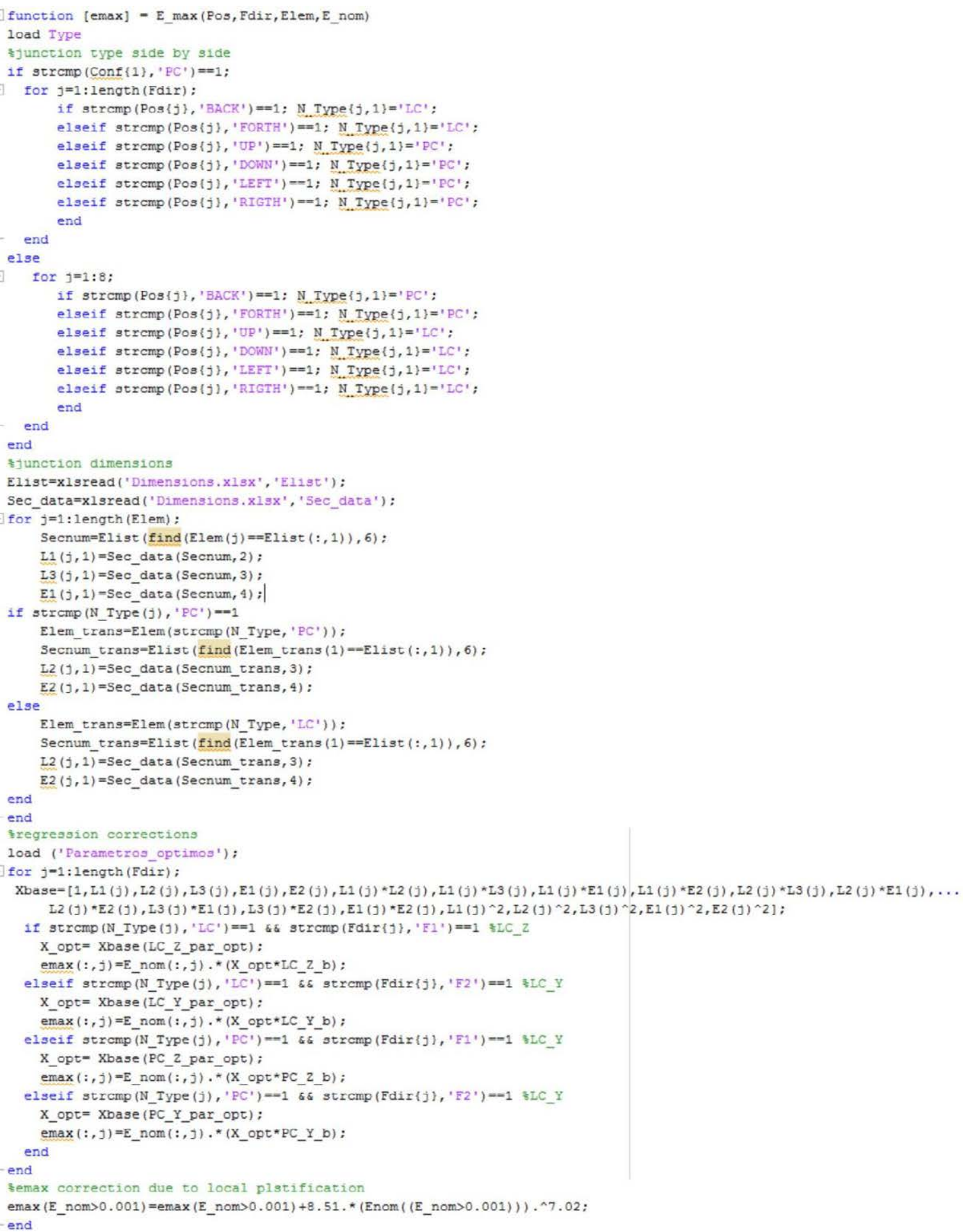

Figure 116 Maximum strains determination function. 


\subsubsection{DETERMINATION OF CYCLES TO FAILURE AND FATIGUE DAMAGE}

This function is called by the main routine right after obtained the corrected maximum strain. Similarly, it is also called for lateral, longitudinal and vertical accelerations in lines 18, 23 and 28 respectively. It performs the calculation of the number of load cycles to failure at each junction side and for each acceleration cycle $\left(N_{i}\right)$ and compares it to the expected number of cycles that the bus structure will withstand over its lifespan $\left(n_{i}\right)$ to determine the fatigue damage.

The scripting of the function is shown in Figure 117. It performs the following sequence of actions with the final purpose of determining the fatigue damage in each junction side.

- Applies the superposition principle to calculate the resulting equivalent maximum strain resulting from loads in direction 1 and 2 for each junction side.

- Calculates mean and alternate strains for each load cycle.

- Determines the resulting fatigue life $\left(N_{i}\right)$ for each couple $\left(\varepsilon_{m}, \varepsilon_{a}\right)$ using the strain life curve determined experimentally in Section 5.3.

- Extracts the number of cycles that the LPTV structure will withstand $\left(n_{i}\right)$ from the Rainflow data of Section 4 and the manually input lifespan of the vehicle (in function lines 63 to $65)$.

- Calculate the damage as the relation between the number of cycles to failure and the number of cycles withstood during the lifespan $\left(n_{i} / N_{i}\right)$, according to the Palmgren-Minner rule (eq. (1.31)).

- Constructs a graphical plot of the damage at each side of the junctions, and for each acceleration cycle $\left(a_{m}, a_{a}\right)$. An example of this plot is shown in Figure 118.

The function returns to the main routine the strain fatigue cycles ( $e_{-}$mean, $\left.e_{-} a l t\right)$ that correspond to the applied acceleration cycles $\left(a_{m}, a_{a}\right)$, the number of cycles to failure for each acceleration cycle ( $\left.N_{-} e_{-} s d\right)$ the damage that each cycle implies to each junction side ( $\left.D \_l i f e\right)$, and the overall damage of each junction side (D_life_tot). 


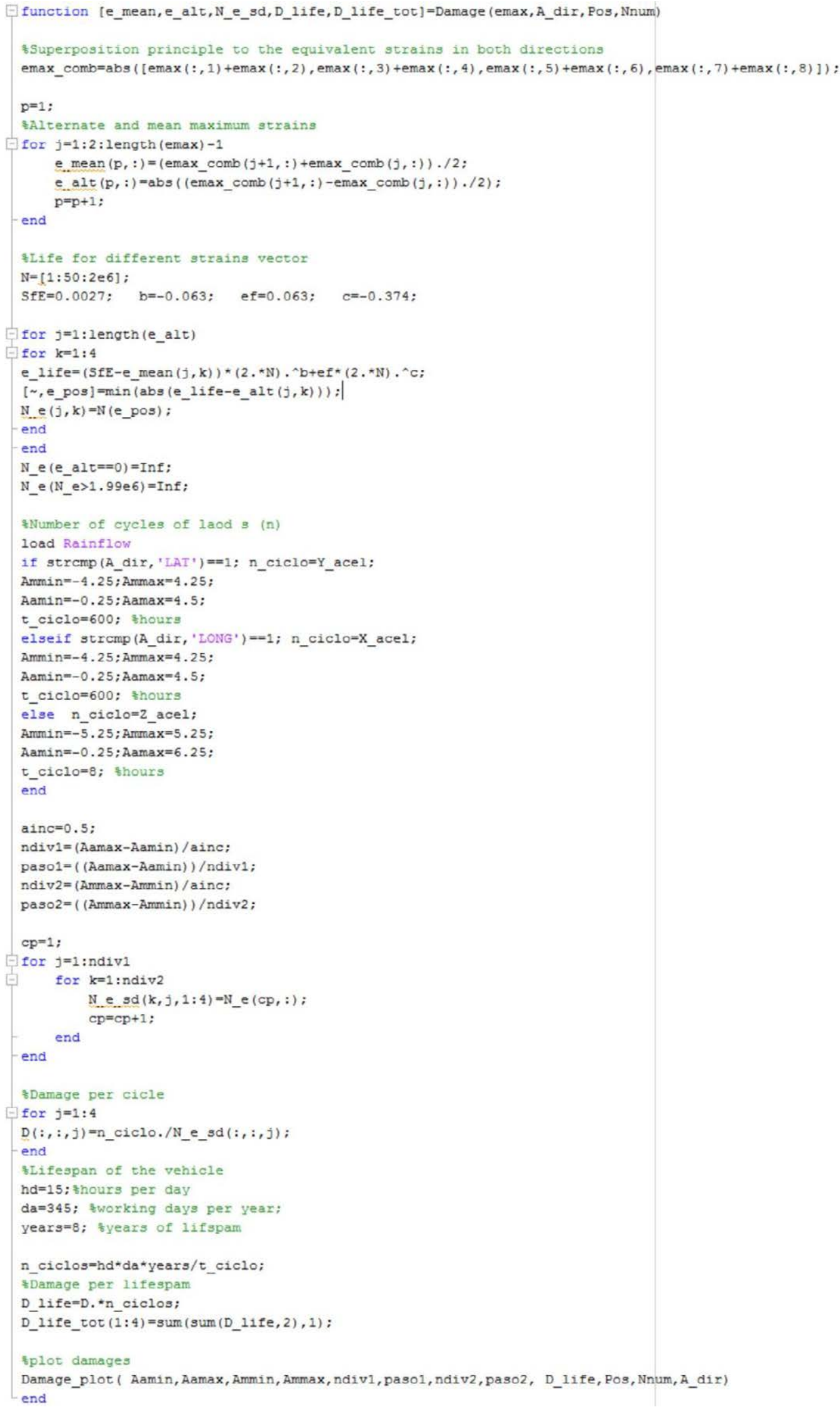

Figure 117 Life and damage calculation function. 


\subsubsection{OUTPUTS}

The outputs that the routine provides are, on one hand, a comma separated .csv text file with the total damage suffered by each junction side (or element adjacent to the junction nodes) that will be later used to plot this damage over the structure in Ansys ${ }^{\circledR}$. On the other hand, it constructs the following plots that give detailed information about the fatigue behavior of each junction:

- Individual damage that each acceleration cycle implies in each junction side. This plot enables to quickly localize the worst damaging load cycles for each junction and evaluate whether this damage may cause fatigue failure $(D>1)$. By way of example, Figure 118 shows this plot for vertical accelerations in the node 8845 used as example. It is noticed that acceleration cycles with higher alternate component cause nonzero damage in the 'UP' and more significantly in the 'BACK' side of the junction with a total damage of 0.099 and 0.549 respectively. In any case, no fatigue failure is expected in any junction side due to vertical accelerations since the total damage is in all cases is below the unity.
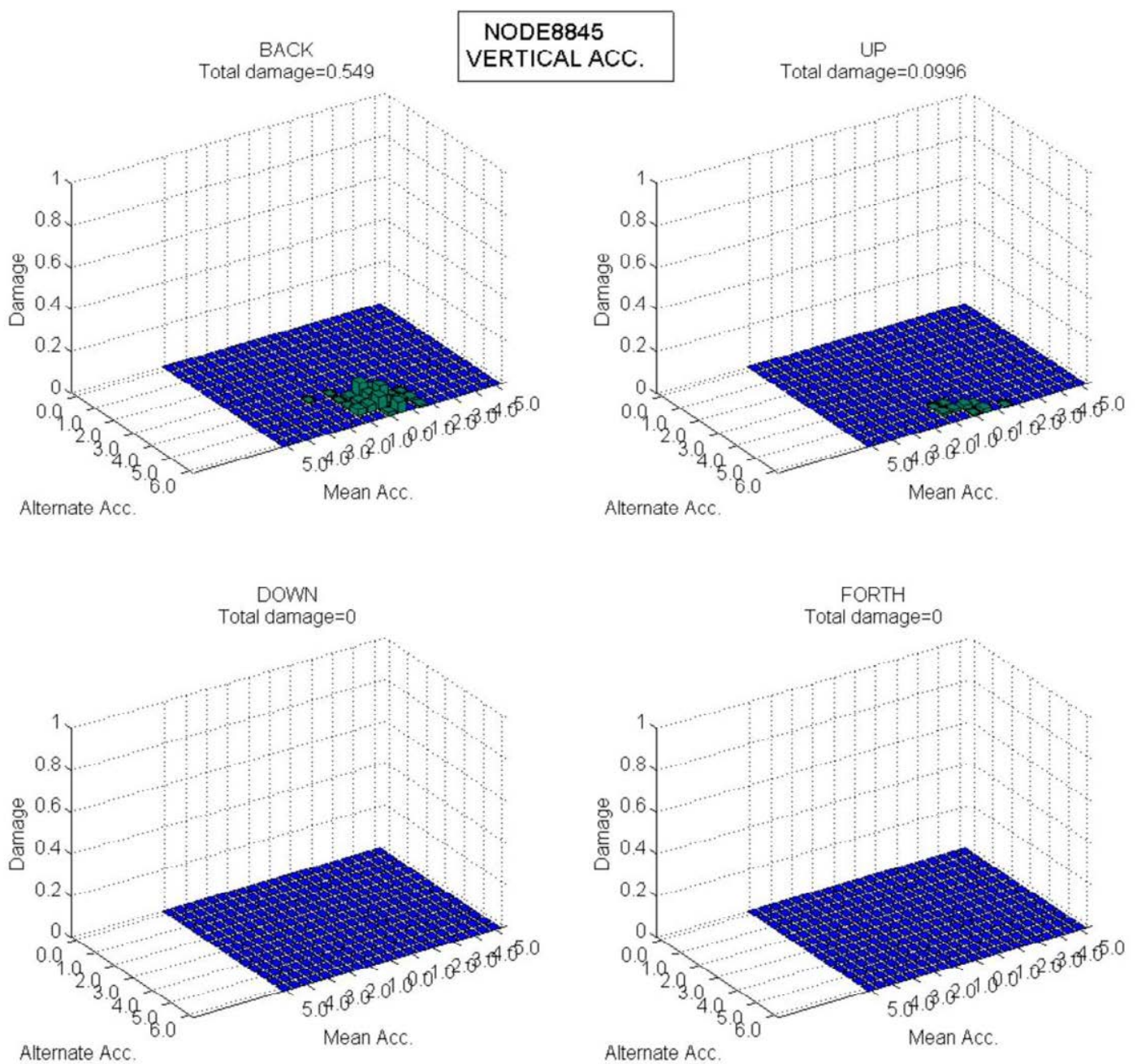

Figure 118 Individual fatigue damage plots for vertical accelerations. 
- Comparative view of the strain level at each junction side and for each acceleration direction. This strain level is determined as the mean of the maximum strains $\left(\varepsilon_{m}+\varepsilon_{a}\right)$ in each case. This graph enable to quickly compare the level of solicitation of each side and acceleration direction. The interpretation of this graphs is mostly qualitative and provides an easy to read information that can be of special interest in case of design improvements of the structure. Figure 119 shows this plot for node 8845, where it is clearly noticed that junction sides situated in the 'UP' and 'BACK' present the highest strain levels, which is in accordance with the individual damage plot shown in Figure 118. Additionally, it is also seen that vertical accelerations provoke the highest strains in these sides of the junction, whereas lateral ones are noted to be more harmful in the other two sides. Finally, it should be kept in mind that this graph, although linked to fatigue damage, does not show a direct relation with it, since fatigue damage also depends upon the number of cycles withstood at each load level.

NODE 8845

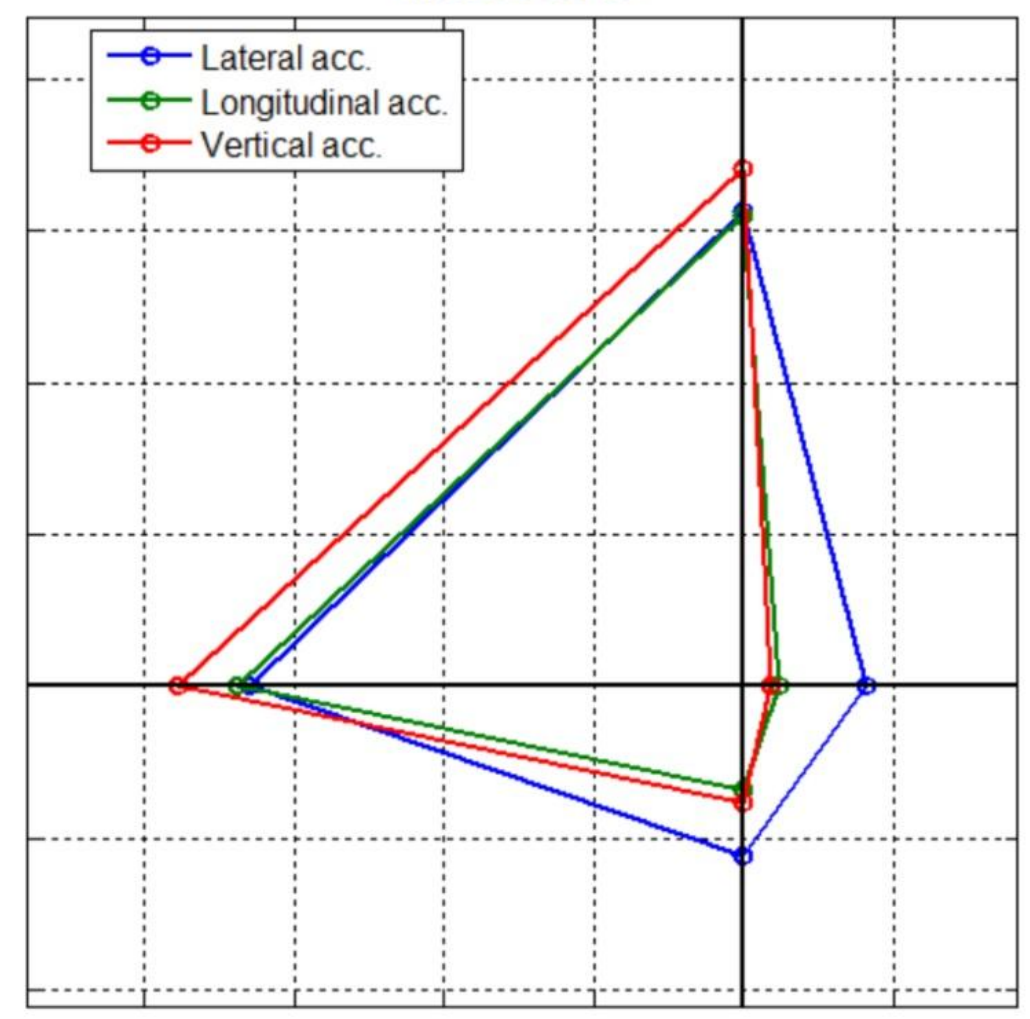

Figure 119 Strain level comparative plot.

- Junction damage overview. This plot gives a compacted view of the total damage suffered by each junction side, providing information about the contribution to the damage of the different acceleration directions. Figure 120 shows this graph for the node used as example, were is noticed that damage only occurs at 'UP' and 'BACK' sides of the junction, and that only vertical accelerations has a contribution to it. Consequently, the total damage of each junction side coincides in this case with the total damage shown in the top of the subplots of Figure 118. 


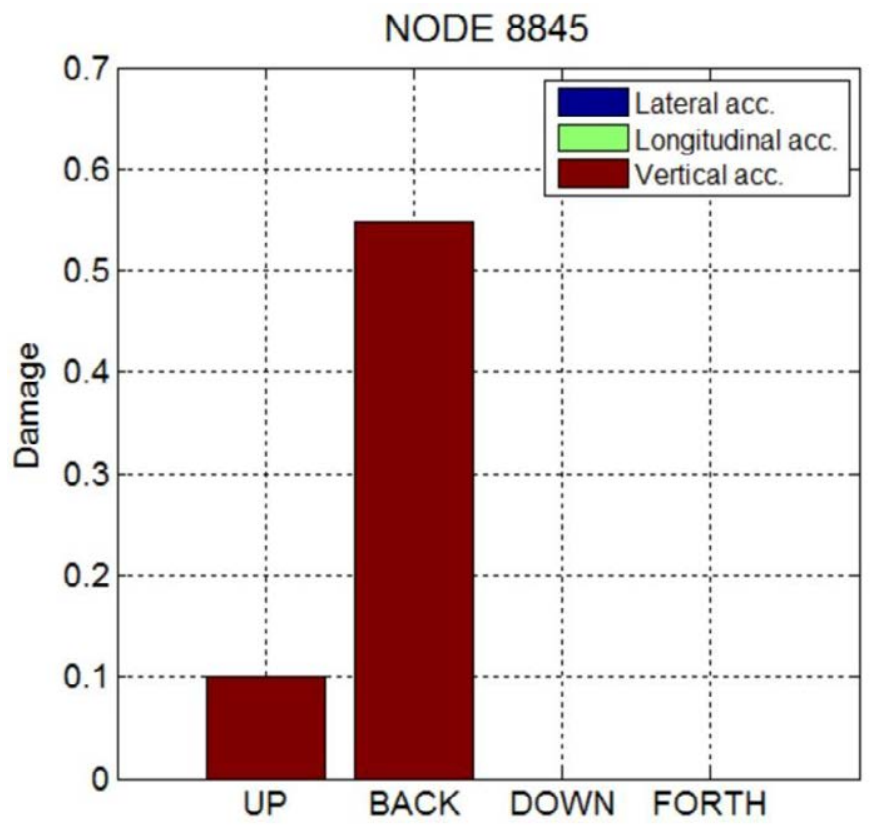

Figure 120 Summarized overview of junction damage.

- Overall plot of the damage of the structure. Is the most global plot, giving a compacted information about the maximum damage of all the studied junctions in a single bar plot, enabling to have a quick overall idea if the quality of the structure design in terms of fatigue. This graph is also of great use for assessing the improvement among the different design iterations in a quick and compacted manner. It employs a color code to distinguish the different damage levels: thus, any junction presenting a maximum damage between 0 and 0.5 will be shown with a green bar, if the damage level lies somewhere between 0.5 to 1 the bar will paper in yellow, and if the damage exceeds the unity (will present failure within the lifespan), this bar will be shown in red color. The overall plot for the studied junctions of the model is shown in Figure 121. It is noticed that only 5 out of 73 studied junctions present fatigue damage, 4 of which this damage is under 0.5 , and only one node shows a damage level over 0.5 . No damage exceeds the unity, so therefore it can be concluded that no fatigue failure is expected during the lifespan if the vehicle in the studied junctions.

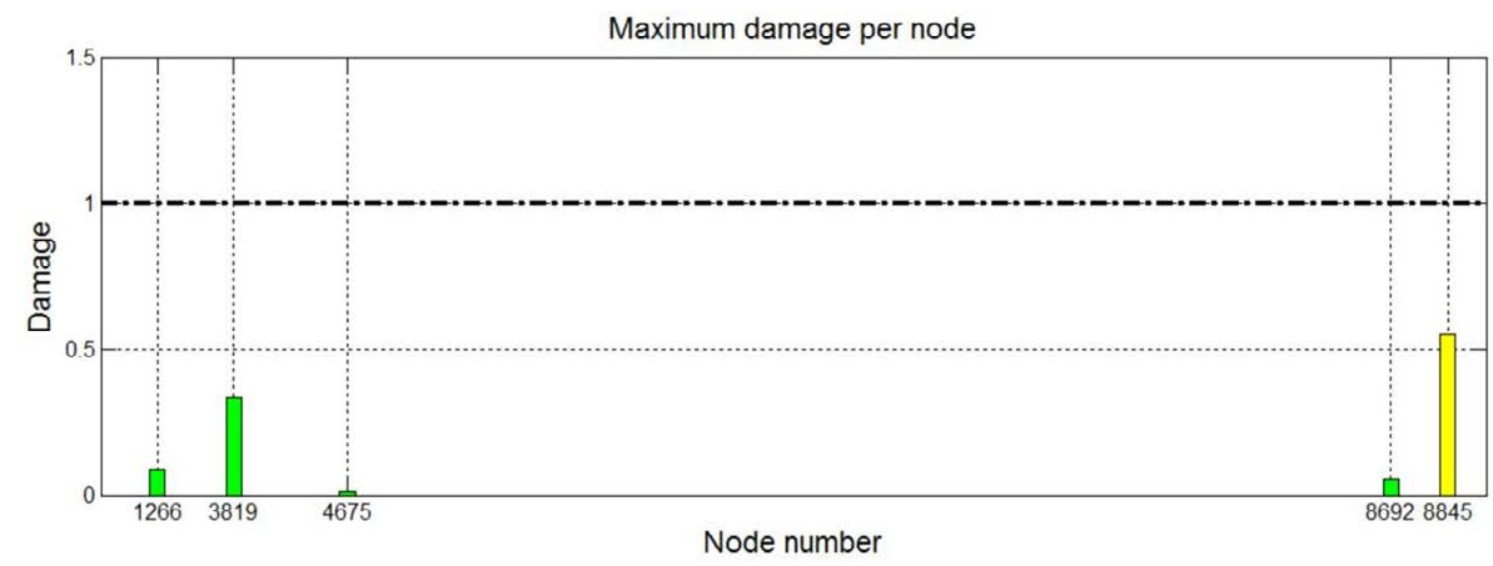

Figure 121 Overall fatigue damage plot. 


\subsection{LOCATION OF DAMAGED JUNCTIONS IN THE STRUCTURE}

The previous subsections have described the corresponding routines and functions developed to assess the fatigue behavior of 'T' welded junctions on the studied LPTV structure in accordance with the methodology presented in Figure 1. Consequently, the evaluation process can be considered completed, since the damage at each junction was provided. Nevertheless, given that the methodology is applied over a simplified finite element model of the structure, it might be of interest to have access to the damage of each junction on the model itself. It would enable localizing most damaged junctions and areas, and thus further investigate possible causes for these damage and take the corresponding actions to improve the design.

For this purpose, an additional Ansys ${ }^{\circledast}$ APDL routine was generated (Figure 122). The routine reads the .csv file stored by the main Matlab $^{\circledR}$ routine, saves it to in an element table that subsequently plots. The plot follows the same color code as in the overall damage plot of Figure 121, adding the blue color for elements with zero damage. The routine also generates a group named 'damagedelem' with the elements of the evaluated junctions that suffer any damage over 0.0001 .

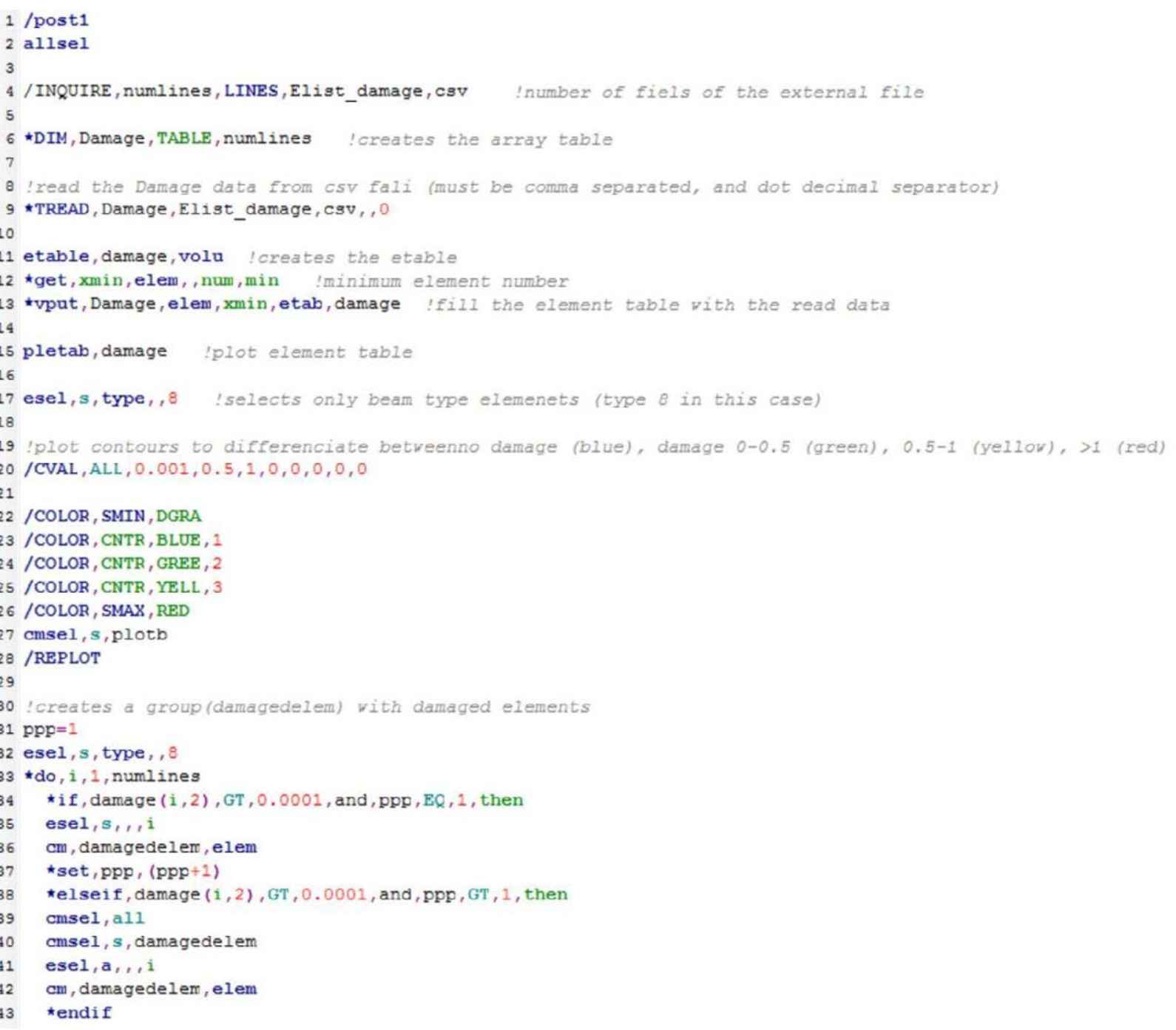

Figure 122 Damage plot Ansys APDL routine. 
In order to ensure the proper functioning of the routine, the output file generated by Matlab ${ }^{\circledR}$ should be copied to the same folder as the finite element model of the bus. Also, line 17 of the routine selects the elements associated with the BEAM188 element type which is element type number 8 in the case of the example. If other model with other element type number for BEAM188 elements is used, this value should be substituted.

The output plot generated by the routine is shown in Figure 123. The 5 junctions with nonzero fatigue damage have been pointed to improve its visibility in the image. In any case, it should be noted that the purpose of the routine is not to generate a fixed capture of the structure, but plotting damaged elements over the Ansys model in order to visualize and evaluate them dynamically.

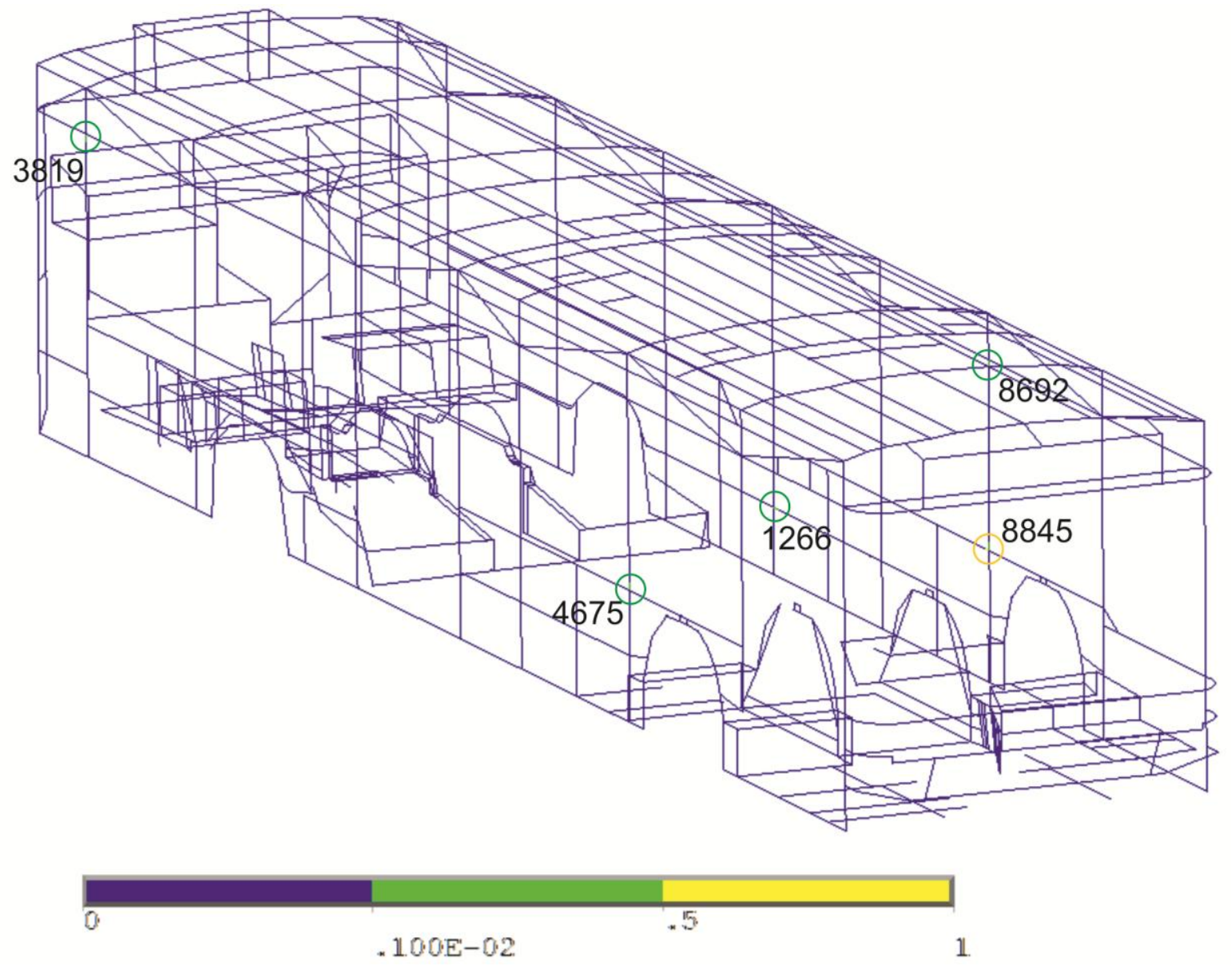

Figure 123 Damaged junctions Ansys plot. 


\section{CONCLUSIONS}

This thesis has been developed with the ultimate goal of providing a methodology to assess the fatigue behavior of LPTV welded body structures that encompasses reliable results with simple finite element modeling. For this purpose, different research activities where needed to be carried out which have also brought significant contributions and findings. They can be divided in the following points, that go in accordance with the structure of the methodology itself:

- An extensive and representative characterization of the dynamic loads suffered by the LPTV structures during operation has been carried out. It was evidenced that the magnitude of these loads is well below the stability limitations of the bus, and thus designing based in these assumptions would lead to non optimized solutions.

Acceleration cycles belonging to longitudinal an lateral accelerations show a more organized distribution since they are consequence of bus maneuvers well controlled by the driver, and therefore address to matters like stability, comfort, or driving efficiency. On the opposite, vertical accelerations arise from road imperfections and thus cannot be fully controlled by the driver.

Finally, vertical accelerations where found to be the ones that show the most severe cycles and thus can potentially cause more fatigue damage on the structure, although it will also depend on the bus structure design.

- A complete structural characterization of the tube beam welded junctions was presented, assessing the material strength of the weld $\mathrm{HAZ}$, the strain concentration behavior of the junctions, and its fatigue resistance. The experiments carried out to these purposes evidenced the capabilities and advantages of using DIC techniques for strain measurements with respect to the classic strain gauges. Its primary benefit is the capacity of supplying full strain fields instead of punctual data, which is of critical importance when the location of the maximum strains is not known in advance, as is the case of the strain concentration around weld beads.

The strain concentration was found to be dependent on the junction type, load direction and load level. The variation of the later is related to the non linear behavior of the material for the highest load levels tested.

It was also observed a clear tendency of the SCF of each junction independently of the load direction or level, i.e. if a junction tends to concentrate high strains in a specific test condition, it will also show the same tendency in the rest of the cases. This behavior, together with the scatter of the results for same test conditions reveals the significant influence of local geometric characteristics of the weld bead on the SCF.

In regard to the fatigue behavior, no significant differences were found between both types of junctions tested. This observation is supported by the fact that both junctions present very similar characteristics with respect to the principal fatigue resistance modifying factors i.e. size, surface finish, temperature, etc. Therefore, the 
fatigue curve provided in the thesis can be employed to study the different types of T-type welded junctions in LPTV structures.

- It was developed a validation process for detailed finite element models of the LPTV welded junctions. Once proven the necessity of modeling the local geometric characteristics of the junctions in order to avoid skewed results due to singularities on the model, an average weld bead shape was determined based on real weld profiles measured with the aid of the DIC technique. The thus obtained weld bead shape was included in the FEM model, which also carefully accounted for the characteristics of the rectangular profile geometry and material.

The results obtained show a slightly conservative tendency of the FEM models with respect to experimental measurements, since the SCF values calculated range between $8.7 \%$ and $17.4 \%$ higher depending on the junction type and load case.

Finally, and using the validated models, it was verified the proportional multiaxial strain state at the vicinity of the maximum strains independently of the load direction. Therefore, consequent fatigue approaches should be employed to assess fatigue behavior (i.e. based on Von Mises equivalent strains).

- As it was already mentioned, beam type elements cannot account for the geometrical details of the welded junction, and therefore are not capable to produce accurate results of the local maximums strains. In this regard, another important contribution of the thesis was to establish a complete correlation between maximum strains obtained with validated detailed junction models and the simplified ones based on beam type elements. This correlation is based on four different regression models depending on the junctions type and load directions, and was proven to be valid for any ' $\mathrm{T}$ ' welded junction independently of its dimensions. The obtained correlation values are themselves a good approximation of SCF of the junction, since the outcomes of the simplified models corresponds to the nominal strain values (i.e. neglecting local strain raising effects). There were observed different tendencies depending on the junction type and load direction. Junctions type $A$ loaded in $Y$ direction was noticed to show higher values with respect to the rest of configurations, over 10 in some cases. On the contrary, Type B junctions, and Type $A$ for loads in $Z$ direction hardy surpass values of 5 . Additionally, Type A junctions revealed to be specially sensitive to geometric variables related to the thicknesses of the profile sections. Such clear a tendency was not observed for type B junctions, although highest regression estimators also correspond to thickness related parameters. These observations evidence that following the common practice of considering a single SCF value attending only to the weld type would lead to significant deviations.

The errors of the regression models for a 95\% confidence interval was 10\% for Type A junction, and $7 \%$ for the other three configurations. It supposes an overall improvement of the accuracy of the simplified LPTV models of 90 to $99 \%$. In any case, it should be pointed out that there are a few junctions showing errors close to $25 \%$, 
which supposes an improvement on the accuracy of approximately $80 \%$. These deviations should be either way taken into account when applying safety factors or design limits during the structure design process.

Finally, the models were proven to operate with the same accuracy regardless the geometry of the cross section, which validates its capacity for all possible ' $T$ ' type junctions.

- In the last section of the thesis, all the components of the methodology developed were brought together and applied in a case study. The routines supplied allow the fatigue assessment of the ' $T$ ' type junctions of a LPTV structure design. The outputs of the routines are thought to give practical and easy to read information about the fatigue damage on each junction and its route cause. This information enables a precise assessment of the most damaged areas and thus to take the corresponding actions to optimize the structure. It should be noted that the routines presented are just an example of the application of the methodology developed with the purpose of automating the application of its different steps. Any other solution is the same valid as long as it follows the methodology presented in Figure 1 and uses the data supplied throughout the thesis.

It is worth to mention that no conservative actions or safety factors have been adopted in the methodology, although detailed welded junctions FEM models were proven to outcome SCF values slightly higher than reality. If a more conservative design solution are desired, a more rigorous design limit can be easily implemented by decreasing the maximum allowable damage on the junctions.

Overall, a complete fatigue assessment methodology for LPTV body structures was presented. It was based on ad-hoc experiments and validations to provide the maximum precision in an already scatter phenomenon as are fatigue and local strain concentration effects. Also, the strain correlation process developed allows to overcome the inherent limitation of FEM between accuracy and computer cost, permitting to obtain accurate results in simplified models. In all, the information supplied in the thesis is considered a powerful tool for design and optimization of LPTV structures in terms of fatigue resistance.

\subsection{FUTURE WORK}

The most direct line of future research that this thesis leaves is related to the extension of the fatigue damage evaluation to any type of welded junction present in an LPTV structure. Whereas many of these junction are ' $T$ ' type, there are other configurations that could be included in the evaluation. This extension could be integrated to the methodology without modifying its structure, and could be approached in two ways:

- Constructing detailed FEM models of the different junction configurations based on the findings of section 4 for ' $T$ ' type junctions (in terms of cross section and weld bead geometry modeling), and using them to develop additional regressions to correlate the maximum strains between detailed and simplified models. 
- Performing a new experimental characterization of the SCF of the different junctions to carry out a new validation process of the detailed models. This specifically validated models would be later used to construct the regression models.

Both solutions would entail very similar computational costs, which is related to the simulation of the detailed models for the different possible dimensional combinations. Additionally, the second option entail an additional validation work in order to ensure accounting for any local behavior that might differ from the studied ' $T$ ' type junctions.

Another future research of notable interest would be to study possible impact of torsional type loads. An specific acquisition system would be needed to be installed in order to record the torsion loads of the sprung mass during a representative period of normal operation. Posterior processing of the acquired data would enable evaluating the magnitude of such torsions and thus assess whether they might have an impact on fatigue life of the body. 


\section{REFERENCES}

1F. Lan, J. Chen, J. Lin. Comparative analysis for bus side structures and lightweight optimization. Proceedings of the Institution of Mechanical Engineers, Part J: Journal of Automobile Engineering, 218 (2004), 1067-1075.

O. Ruíz, E. Ramirez, V. Jacobo, R. Schouwenaars, A. Ortiz. Efficient optimisation of the structure of a passenger bus by iterative finite element models with increasing degrees of complexity. (Rio de Janeiro 2012), 3rd International Conference on Engineering Optimization.

H. Hu, C. Yang, J. Wang. Development and validation of finite element model for the welded structure of transit bus. International Journal of Heavy Vehicle Systems, 19, 4 (2012), 371-388.

SJ. Park, WS Yoo. Rollover analysis for the body section structure of a large bus using beam and non-linear spring elements. Proceedings of the Institution of Mechanical Engineers, Part D: Journal of Automobile Engineering, 222, 6 (February 2008), 955-962.

R. Su, L. Gui, Z, Fan. Multi-objective optimization for bus body with strength and rollover safety constrains based on surrogate models. Structural and multidisciplinary optimization, 44, 3 (Februery 2011), 431-441.

J. Pérez, E. Alcalá, JM. López. Experimental characterization of the probability density function of the operating loads supported by urban buses in Madrid, Spain. Proceedings of the Institution of Mechanical Engineers, Part D: Journal of Automobile Engineering, 228, 11 (2014), 1315-1329.

A. Bruton, C. Glennie, K. Schwarz. Differentiation for High-Precision GPS Velocity and Acceleration Determination. GPS Solutions, 2, 4 (1999), 7-21.

A. Fatemi, L. Yang. Cumulative fatigue damage and life prediction theories: a survey of the state of the art for homogeneous materials. International Journal of Fatigue, 20, 1 (1998), 9-34.

Y.L. Lee, J. Pan, R.B. Hathaway, M.E. Barkey. Fatigue Testing and Analysis (Theory and Practice). Elsevier Butterworth-Heinemann, 2005.

Albert, W.A.J. Über treibseile am Harz. Archiv für Mineralogie, Geognosie, Bergbau und Hüttenkunde, 10 (1837), 215-234.

Rankine, W.J.M. On the causes of the unexpected breakage of the journals of railway 
axles; and on the means of preventing such accidents by observing the law of continuity in their construction. Minutes of the Proceedings, 2 (January 1843), 105-107.

Braithwaite, F. On the fatigue and consequent fracture of metals. Minutes of the Proceedings, 13 (1854), 463-467.

Wöhler, A. Bericht fiber die Versuche, welche auf der königl. Niederschlesischmiirkischen eisenbahn mit Apparaten zum Messen der Biegung und Verdehung von Eisenbahnwagenachsen w/ihrend der Fahrt angestellt wurden. Zeitschrift fiir Bauwesen (1858), 641-652.

Spangenberg, L. Über das verhalten der metalle bei wiederholten anstrengungen. 1875.

J.A. Ewing, J.C.W. Humfrey. The fracture of metals under repeated alternations of stress. Philosophical Transactions of the Royal Society of London. Series A, Containing Papers of a Mathematical or Physical Character (1903), 241-250.

Basquin, O.H. The exponential law of endurance tests. In Proc. Astm, 10 (1910), 625630.

R.A. MacGregor, W.S. Burn, F. Bacon. The Relation of Fatigue to Modern Engine Design. The North East Coast Institution of Engineers and Shipbuilders, London, 1935.

Palmgren, A. Die lebensdauer von kugellagern. Zeitschrift des Vereins Deutscher Ingenieure, 68, 14 (1924), 339-341.

Miner, M.A. Cumulative damage in fatigue. Journal of applied mechanics, 12, 3 (1945), 159-164.

Gerber, H. Bestimmung der zulässigen spannungen in eisen-constructionen. Z. Bay. Arch. Ing. Ver., 6 (1874), 101.

Goodman, J. Mechanics applied to engineering. Longmans Green (1899).

C.R. Soderberg, V. Sweden. Factor of safety and working stress. Trans. Am. Soc. Test Matls, 52, 2 (1930), 13-28.

Q. Bader, E. Kadum. Mean Stress Correction Effects On the Fatigue Life Behavior of Steel Alloys by Using Stress Life Approach Theories. International Journal of Engineering \& Technology, 14, 4 (2014), 50-58. 
Weibull, W. A statistical theory of the strength of materials. Generalstabens litografiska anstalts förlag (Royal Swedish Academy of Engineering Sciences), 151 (1939).

Weibull, W. Static strength and fatigue properties of threaded bolts. FFA, Report 59 (1955).

Weibull, W. Scatter in fatigue life of 24-St cladded plates. (Cranfield 1955), Minutes of the 3rd ICAF-Conference.

Coffin, L.F. A study of the effect of cyclic thermal stresses on a ductile metal. American Society of Mechanical Engineers, 76, 931-950 (1954).

Manson, S.S. Behavior of materials under conditions of thermal stress. NACA, TN 2933 (1953), 636-670.

Peterson, R.E. Stress concentration design factors. John Wiley, New York, 1953.

Irwin, G.R. Handbuch der Physik. Springer Verlag, 6 (1958), 551.

Elber, W. Fatigue crack propagation: some effects of crack closure on the mechanisms of fatigue crack propagation and cyclic tensile loading. Doctoral dissertation, 1968.

Force, US Air. Military specification airplane damage tolerance requirements. MIL$83444,1974$.

D.P. Rooke, D.J. Cartwright. Stress intensity factors. Her Majesty's Stationery Office, London, 1974.

Goodmann, J. Mechanics Applied to engineering. Longman-Green, London, 1899.

Soderberg, C.R. Factor of Safety and Working Stress. Transactions of the American Society of Mechanical Engineers, 1, (Part APM-52-2) (1930), 13-28.

Gerber, W.Z. Bestimmung der Zulossigen Spannungen in Eisen Constructionen. Z. Bayer. Archit. Ing. Ver., 6 (1874), 101.

Morrow, J. D. Fatigue Design Handbook. Society of Automotive Engineers, 4 (1968), 21-29.

K.N. Smith, P.Watson, T.H. Topper. A stress-strain function for fatigue of emtals. Journal of Materials JLMSA, 5, 4 (1970), 767. 
Marin, J. Mechanical Behaviorof Engineering Materials. (New Jersey 1962), PrenticeHall.

Norton, R.L. Machine Design: An Integrated Approach (3o ed.). Pearson International Edition, New Yerseey, 2006.

J.E. Shigley, C.R. Mischke. Diseño en Ingeniería Mecánica. McGraw Hill, New York, 1990.

J.H. Faupel, F.E. Fisher. Engineering Design (2o edition). John Wiley \& Sons, New york, 1981.

J.A. Bannantine, J.J. Comer, J.L. Handrock. Fundamentals of metal fatigue analysis. Prentice-Hall, New Jersey, 1990.

R.I. Stephens, A. Fatemi, R.R. Stephens, H.O. Fuchs. Metal Fatigue in Engineering (2o edition). John Wiley \& Sons, New York, 2001.

R.C. Rice, B.N. Leis, D. Nelson. Fatigue design handbook (3rd edition). Society of Automotive Engineers, 1997.

E1049, ASTM Standard. Standard Practices for Cycle Counting in Fatigue Analysis. Philadelphia, 1985.

C. Amzallag, J.P. Gerey, J.L. Robert, J. Bahuaud. Standarization of the rainflow counting method for fatigue analysis. International Journal of Fatigue, 16, 287-293 (1994).

C.H. Mclnnes, P.A. Meehan. Equivalence of four-point and three-point rainflow cycle counting algorithms. International Journal of Fatigue, 30 (2008), 547-559.

M. Matsuishi, T. Endo. Fatigue of metals subjected to varying stress. ( 1968), Japanese Society of Mechanical Engineering.

Taylor, R.L. Zienkiewicz and O.C. The finite element method: solid mechanics (Vol.2). Butterworth-heinemann, 2000.

Reddy, J.N. An introduction to the finite element method. 3th edition. McGraw-Hill, New York, 2005.

Askenazi, V. Adams \& A. Building better products with finite element analysis. Onword press, Oostende, 1999. 
F. Badea, F. Aparicio, E. Alcalá. Metodos numéricos y estadísticos de caracterizacion de uniones tubulares soldadas para su aplicacion en modelos de elementos finitos de estructuras de vehículos. Universidad Politécnica de Madrid, Madrid, 2014.

O. Ruiz, E.I. Ramirez, V.H. Jacobo, R. Schouwenaars, A. Ortiz. Efficient optimisation of the structure of a passenger bus by iterative finite element models with increasing degrees of complexity. (Rio de Janeiro (Brazil) 2012), 3rd International Conference on Engineering Optimization.

S. Manokruang, S. Butdee. Methodology of Bus-Body Structural Redesign for Lightweight Productivity Improvement. Asian International Journal of Science and Technology in Production and Manufacturing Engineering, 2, 2 (2009), 79-87.

W. Ramberg, W.R. Osgood. Description of stress-strain curves by three parameters. NACA, 1943.

ASTM. Standard practice for strain-controlled fatigue testing. American Society for Testing and Materials, 1998.

Lopez, Z. Correlations among tensile and cyclic deformation properties for steels and implications on fatigue life predictions. University of Toledo, 2012.

T.E. Langlais, J.H. Vogel. Overcoming Limitations of the Conventional Strain-Life Fatigue Damage Model. Journal of Engineering Materials and Technology, 118, 1 (1996), 103-108.

C.R. Williams, Y.L. Lee, J.T. Rilly. A practical method for statistical analysis of strainlife fatigue data. International Journal of Fatigue, 25, 5 (2003), 427-436.

V.T. Troshchenko, L.A. Khamaza. Strain-life curves of steels and methods for determining the curve parameters. Part 1. Conventional methods. Strength of materials, 42 , 6 (2010), 64-659.

Neuber, $\mathrm{H}$. Theory of stress concentration for shear-strained prismatical bodies with arbitrary nonlinear stress-strain law. Journal of Applied Mechanics, 28, 4 (1961), 544-550.

H. Hu, C.L. Yang, J. Wang. Development and validation of finite element model for the welded structure of transit bus. International Journal of Heavy Vehicle Systems, 19, 4 (2012), 371-388.

G. glinka, A. Newport. Universal features of elastic notch-tip stress fields. 
International Journal of Fatigue, 9, 3 (1987), 143-150.

Fricke, W. Guideline for the fatigue assessment by notch stress analysis for welded structures. International Institute of Welding, 2008.

F.R. Mashiri, X.L. Zhao, P. Grundy. Stress concentration factors and fatigue behaviour of welded thin-walled CHS-SHS T-joints under in-plane bending. Engineering Structures, 26, 13 (2004), 1861-1875.

W.M. Gho, F. Gao. Parametric equations for stress concentration factors in completely overlapped tubular K (N)-joints. Journal of Constructional Steel Research, 60, 12 (2004), 1761-1782.

M.A Sutton, J.J. Orteu, H. Schreier. Image correlation for shape, motion and deformation measurements: basic concepts, theory and applications. Springer Science \& Business Media, 2009.

P. Lava, S. Cooreman, S. Coppieters, M. DeStrycker, D. Debruyne. Assessment of measuring errors in DIC using deformation fields generated by plastic FEA. Optics and Lasers in Engineering, 47 (2009), 747-753.

Gombor, B. Dynamic analysis of a bus body frame: determination of the loads and stresses. Vehicle system dynamics, 43, 11 (2005), 807-822.

Wideberg, J.P. Simplified method for evaluation of the lateral dynamic behaviour of a heavy vehicle. International Journal of Heavy Vehicle Systems, 11, 2 (2004), 195-207.

Snyder, J.P. Map Projections A Working Manual. U.S. Geological Survey Professional Paper 1395, Washintong, 1987.

Z. Boumerzoug, C. Derfouf, T. Baudin. Effect of Welding on Microstructure and Mechanical Properties of an Industrial Low Carbon Steel. Engineering, 2 (July 2010), 502506.

R. Kacar, K. Kökemli. Effect of controlled atmosphere on the mig-mag arc weldment properties. Materials and Design, 26 (2005), 508-516.

Z.L. Zhang, M. Hauge, C. Thaulow, J. Ødegard. A notched cross weld tensile testing method for determining true stress-strain curves for weldments. Engineering Fracture Mechanics, 69 (2002), 353-366.

Maddox, S.J. Fatigue strength of welded structures. Woodhead publishing, 1992. 
International, ASTM. E111-04: Standard Test Method for Young's Modulus, Tangent Modulus, and Chord Modulus. ASTM International, Pennsylvania, 2011.

E646-16, ASTM. Standard Test Method for Tensile Strain-Hardening Exponents ( $n$ Values) of Metallic Sheet Materials. ASTM International, 2016.

10, A370 -. Standard Test Methods and Definitions for Mechanical Testing of Steel Products. ASTM International, Pennsylvania, 2010.

J.A. Pérez, E. Alcalá, A. Martín. Elaboración y validación de modelos FEM de uniones soldadas mediante correlación digital de imágenes. (La Plata (Argentina) 2013).

W.L. Oberkampf, M.F. Barone. Measures of agreement between computation and experiment: Validation metrics. Journal of Computational Physics, 217 (2006), 5-36.

A.T. Beck, C.R.A. da Silva. Timoshenko versus Euler beam theory: Pitfalls of a deterministic approach. Structural Safety, 33 (2011), 19-25.

I. Nonaka, S. Setowaki, Y. Ichikawa. Effect of load frequency on high cycle fatigue strength of bullet train axle steel. International Journal of Fatigue, 60 (2013), 43-47.

Robert, A.H. Wood and M.E. Damage Tolerant Design Handbook: Guidelines for the analysis and Design of Damage Tolerant Aircraft. USAF, 1979.

ASTM. Standard practice for statistical analysis of linear or linearized stress-life (S-N) and strain-life (e-N) fatigue data. American Society for Testing and Materials, 2010.

Manson, S.S. Discussion of reference 16 en the Journal of Basic Engineering. Journal of Basic Engineering, 84, 4 (1962).

P.C. Paris, M.P. Gomez and W.E. Anderson. A rational analytic theory of fatigue. The Trend in Engineering, 13, 1 (1961), 9-14.

R.C. Juvinall, K.M. Marshek. Fundamentals of Machine Component Design (3o ed.). Wiley \& Sons, New York, 2000.

Haigh, B.P. Report British Association (1915), 163-170.

Ludwik, P. Über den Einfluß der Deformationsgeschwindigkeit bei bleibenden Deformationen mit besonderer Berücksichtigung der Nachwirkungserscheinungen. Physikalische Zeitschrift, 10 (1909), 411-417. 
Hollomon, J.H. Transactions of the American Institute of Mining, Metallurgical and Petroleum Engineers (1949), 268-290.

Swift, H. Plastic instability under plane stress. Journal of the Mechanics and Physics of Solids, 1, 1 (1952), 1-18.

Hou, C.Y. Fatigue analysis of welded joints with the aid of real three-dimensional weld toe geometry. International Journal of Fatigue, 29 (2007), 772-785.

Mert, T. Finite Element Analysis of Effect of Weld Toe Radius and Root Gap on Fatigue Life of T-Fillet Welded Joint. Conference of the International Journal of Arts and Sciences ISSN: 1943-6114, 1 (2009), 119-127.

A. Fouathia, S. Meziani, M. Gaci. Numerical Simulation of stresses in welded T tubular structure. International Journal of Scientific Research \& Engineering Technology, 1 (2014), 17.

O. Doerk, W. Fricke, C. Weissenborn. Comparison of different calculation methods for structural stresses at welded joints. International Journal of Fatigue, 25 (2003), 359-369.

Fricke, W. Recommended Hot Spot Analysis Procedure for Structural Details of FPSOs and Ships Based on Round-Robin FE Analyses. Proceedings of the Eleventh International Offshore and Polar Engineering Conference, IV (2001).

D. Shinabuth, C. Benyajati, S. Lapapong, M. Pimsarn, M. Okuma. Finite Element Analysis of an Electric Bus Body Structure in Real Drivin Conditions. International Conference on Mechanical Engineering, CST 1020 (October 2012).

C.C. Liang, G.N. Le. Lightweight optimization of bus frame structure considering rollover safety. WIT Transactions on Ecology and The Environment, 155 (2012).

Commision, European. Statistical Pocketbook- EU Transport in figures. Luxembourg: Publications Office of the European Union, Bietlot (Belgium), 2016.

Bauschinger, J. On the Change of the Position of the Elastic Limit of Iron and Steel under Cyclic Variations of Stress. Mitt. Mech.Tech. Lab, 13, 1 (1886).

D. Radaj, C.M. Sonsino, W. Fricke. Fatigue assessment of welded joints by local approaches. Woodhead publishing, Cambridge, 2006. 